ORNL/TM-2008/156

\title{
The Integrity of ACSR Full Tension Single-Stage Splice Connector at Higher Operation Temperature
}

September, 2008

Prepared by John Jy-An Wang, Edgar Lara-Curzio, Tom J. King Jr. Oak Ridge National Laboratory

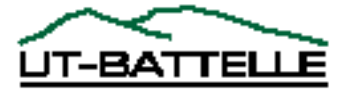




\title{
DOCUMENT AVAILABILITY
}

Reports produced after January 1, 1996, are generally available free via the U.S. Department of Energy (DOE) Information Bridge.

Web site http://www.osti.gov/bridge

Reports produced before January 1, 1996, may be purchased by members of the public from the following source.

\author{
National Technical Information Service \\ 5285 Port Royal Road \\ Springfield, VA 22161 \\ Telephone 703-605-6000 (1-800-553-6847) \\ TDD 703-487-4639 \\ Fax 703-605-6900 \\ E-mailinfo@ntis.fedworld.gov \\ Web site http://www.ntis.gov/support/ordernowabout.htm
}

Reports are available to DOE employees, DOE contractors, Energy Technology Data Exchange (ETDE) representatives, and International Nuclear Information System (INIS) representatives from the following source.

Office of Scientific and Technical Information

P.O. Box 62

Oak Ridge, TN 37831

Telephone 865-576-8401

Fax 865-576-5728

E-mail reports@adonis.osti.gov

Web site http://www.osti.gov/contact.html

\begin{abstract}
This report was prepared as an account of work sponsored by an agency* of the United States Government. Neither the United States government nor any agency thereof, nor any of their employees, makes any warranty, express or implied, or assumes any legal liability or responsibility for the accuracy, completeness, or usefulness of any information, apparatus, product, or process disclosed, or represents that its use would not infringe privately owned rights. Reference herein to any specific commercial product, process, or service by trade name, trademark, manufacturer, or otherwise, does not necessarily constitute or imply its endorsement, recommendation, or favoring by the United States Government or any agency thereof. The views and opinions of authors expressed herein do not necessarily state or reflect those of the United States Government or any agency thereof.
\end{abstract}

\footnotetext{
* This report describes research jointly sponsored by the Electric Power Research Institute (EPRI) and DOE Office of Electricity Delivery and Energy Reliability.
} 
Materials Science and Technology Division

\title{
THE INTEGRITY OF ACSR FULL TENSION SINGLE-STAGE SPLICE CONNECTOR AT HIGHER OPERATION TEMPERATURE
}

\author{
John Jy-An Wang, Edgar Lara-Curzio, Tom J. King Jr. \\ Oak Ridge National Laboratory
}

Date Published: September 2008

\author{
Prepared by \\ OAK RIDGE NATIONAL LABORATORY \\ P.O. Box 2008 \\ Oak Ridge, Tennessee 37831-6285 \\ managed by \\ UT-Battelle, LLC \\ for the \\ U.S. DEPARTMENT OF ENERGY \\ under contract DE-AC05-00OR22725
}




\section{ABSTRACT}

Due to increases in power demand and limited investment in new infrastructure, existing overhead power transmission lines often need to operate at temperatures higher than those used for the original design criteria. This has led to the accelerated aging and degradation of splice connectors. It is manifested by the formation of hot-spots that have been revealed by infrared imaging during inspection. The implications of connector aging is two-fold: (1) significant increases in resistivity of the splice connector (i.e., less efficient transmission of electricity) and (2) significant reductions in the connector clamping strength, which could ultimately result in separation of the power transmission line at the joint. Therefore, the splice connector appears to be the weakest link in electric power transmission lines. This report presents a protocol for integrating analytical and experimental approaches to evaluate the integrity of full tension singlestage splice connector assemblies and the associated effective lifetime at high operating temperature.

\section{Keywords}

Overhead Transmission

Conductors

Compression Fittings

Aging

Annealing

High temperature operation

Single Stage Splice Connector Fitting

Thermal Cycling 



\section{CONTENTS}

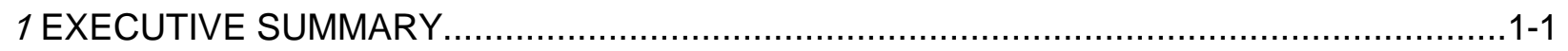

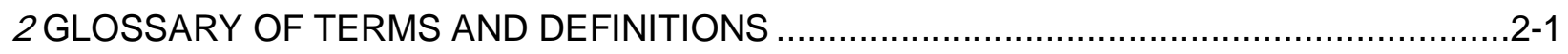

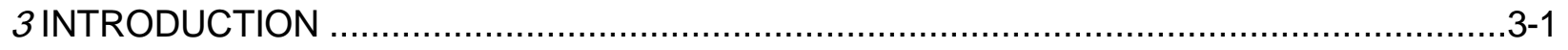

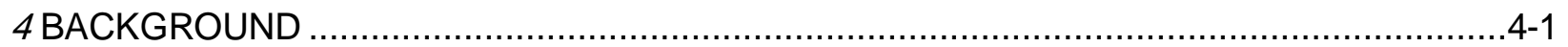

4.1 Background of Thermo-mechanical Loadings in HTLS Conductors............................4-1

4.2 The Impact of Material Aging and Microstructural Evolution to Lifetime Estimate of

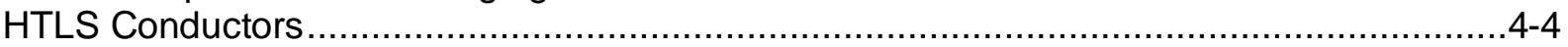

4.2.1 Joint interfaces between splice and conductor ..............................................4-4

4.2.2 Aluminum anodizing and its relation to the splice connector................................4-4

4.2.3 Issues regarding fatigue damage associated with AC current .............................4-4

4.2.4 Electromigration microstructural damage of the conductor ................................ $4-5$

4.2.5 Flaw-induced localized high temperature spots ......................................... 4-5

4.3 The Impact of Bending Moment Effect to the Integrity of ACSR Conductor at Suspension Ends during High Temperature Operation ................................................... $4-6$

4.3.1 Cable Continues Through the Suspension Ends ................................................ 4-7

4.3.2 Potential bending moment induced damage at suspension ends during high

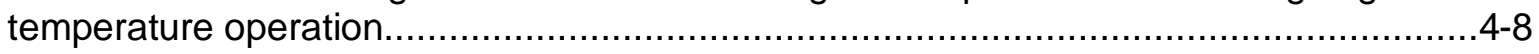

4.4 Mixed-mode Loadings Condition of a Twisted Conductor Wire Bundle .........................4-9

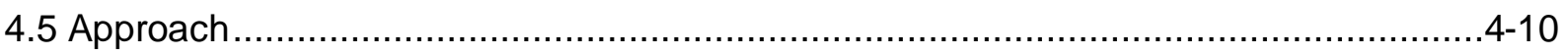

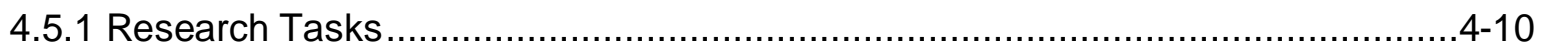

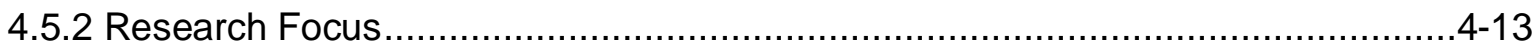

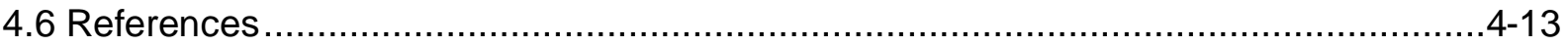

5 THERMAL-MECHANICAL PROPERTIES EVALUATION FOR ACSR SSC SYSTEM

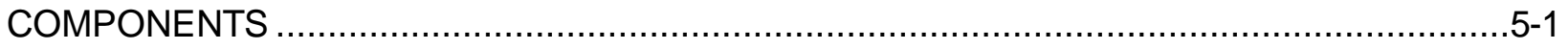

5.1 The Preparation of Test Set-Up for Tensile, Creep, Fatigue, and Thermo-

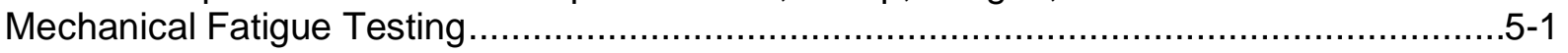

5.1.1 Test Set-up for Tensile and Fatigue Testing at High Temperatures.......................5-2 
5.1.2 Test Set-up of ATS Creep Machines and Cantilever Creep Tester......................5-6

5.2 PILOT TENSILE TESTS ON 1350-H19 CONDUCTOR MATERIAL............................5-7

5.2 .1 Manufacturing 1350-H19 Aluminum Material ........................................... $5-7$

5.2.2 Pilot Testing Set-up and the Associated Specimen Grip Design of the Received Aluminum 1350 Material............................................................... $5-8$

5.2.3 New Specimen Designs for Tensile and Fatigue Testing of 1350-H19 Rod.........5-10

5.2.4 Tensile Test Results for the $1350-\mathrm{H} 19$ Rod Material at $20^{\circ} \mathrm{C}, 120^{\circ} \mathrm{C}$, and

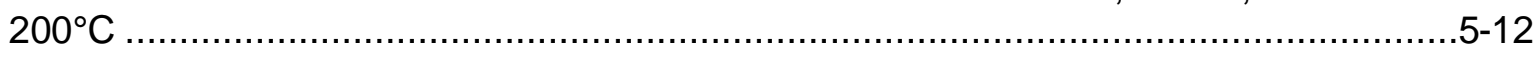

5.3 Drake Conductor SCC System Mechanical Properties ........................................... 5-14

5.3.1 Specimen Preparation for SSC System Components...................................5-15

5.3.2 In-situ Mechanical Property Evaluation of ACSR SSC Fittings ..........................5-15

5.3.3. Tensile and Compression Test of 3003-H183 Aluminum Splice Sleeve .............5-16

5.3.4 Tensile Test of 6061-T6 Aluminum Core-grip.............................................5-18

5.4 Material Flow Properties Developed for Finite Element Analyses .............................5-18

5.5 Creep Test Results..................................................................................... $5-20$

5.6 Isothermal Fatigue Test Results ................................................................ $5-21$

5.7 Thermal-Mechanical Fatigue Test Results..................................................... $5-22$

5.8 SEM Microstructure Examinations ............................................................ $5-23$

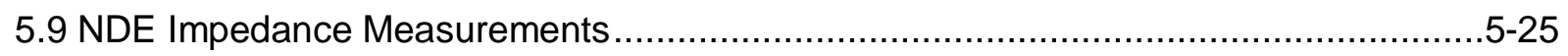

6 INTEGRITY OF ACSR SINGLE-STAGE SPLICE CONNECTOR SYSTEM \& ITS

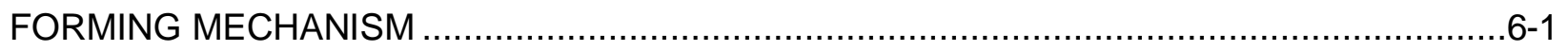

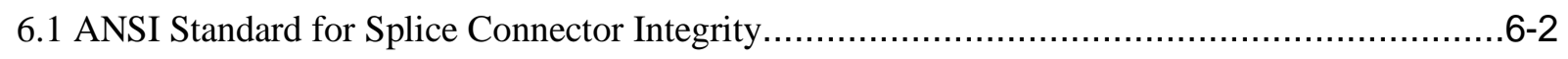

6.2 Analytical Evaluation of Single-Stage Splice Connector Forming..............................6-3

6.2.1 Descriptions of ACSR Drake SSC System and Its Forming Procedures...............6-3

6.2.2 Load Transfer within A SSC Fitting ...................................................... $6-5$

6.3 FEM Residual Stress Analysis for Core-grip Section .........................................6-6

6.3.1 FEM Analysis Results for 3P, Vert, and 15D Core-grip Positions ......................6-6

6.4 FEM Residual Stress Analysis for the Conductor Section......................................6-10

6.5 In-Situ Non-Destructive Evaluation of the Residual Stresses within A Crimped

Splice Connector .................................................................................... $6-13$

6.5.1 Basic Concept of Measuring Residual Strain by Neutron Diffraction Strain

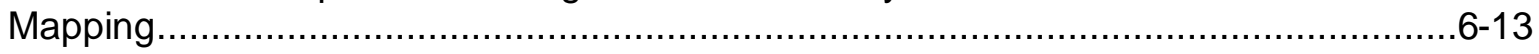

6.5.2 Experimental Set-up and the Test Results ...........................................6-15

6.5.3 Comparison of Residual Stress Data from FEM Analyses and HFIR Neutron

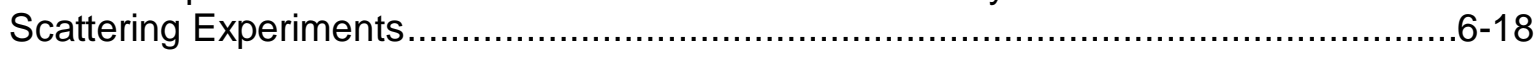

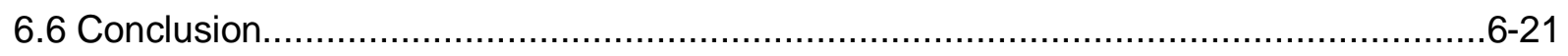




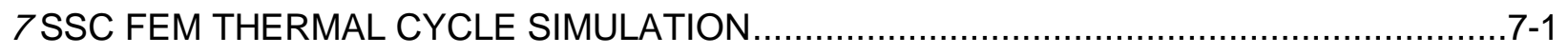

7.1 Thermal-Mechanical Evaluation for SSC Systems ...............................................

7.2 Temperature Profiles within TSC and SSC Fittings............................................

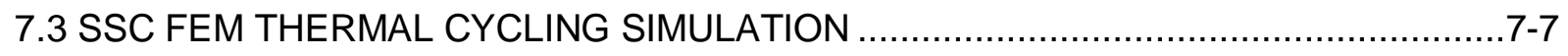

7.3.1 Thermal Cycle Simulation for SSC Conductor Section ...................................... $7-9$

7.3.2. Thermal Cycle Simulation for Core-Grip Section .......................................... $7-12$

7.3.3. Consideration of Single Iteration per Target Temperature ………..................... $7-14$

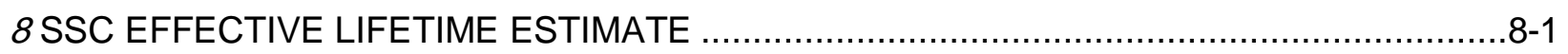

8.1 General Formulation for SCC Lifetime Estimate …............................................ $8-1$

8.2 Calculation of the Shear Resistance Force in SSCs ................................................ $8-2$

8.2.1 Confirmatory structure verification................................................................

8.3 SSC Effective Lifetime Predictions …………................................................. 8

8.3.1 Governing equation of SSC shear resistance capacity ...................................... $8-6$

8.3.2 Governing equation of SSC surface temperature history .................................. 8 -9

8.3.3 Demonstration of SSC effective lifetime prediction ....................................... $8-12$

8.3.3.1 Using SSC shear resistance capacity for SSC lifetime estimate ..................8-12

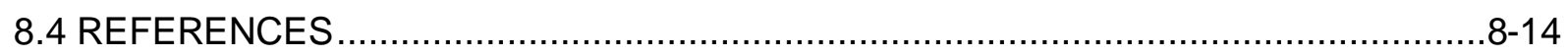

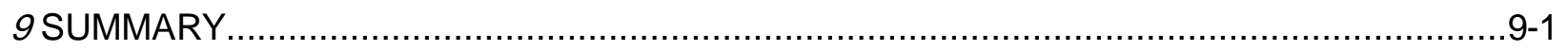

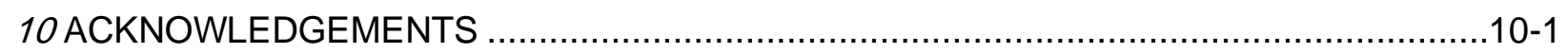

$A$ BENDING MOMENT EVALUATION FOR POWER TRANSMISSION LINES ……............ A-1

A.1 Bending Moment Evaluation Protocols ............................................................ A-2

A.1.1 Theoretical Bending Moment Evaluation Based on a Tie Rod with Lateral

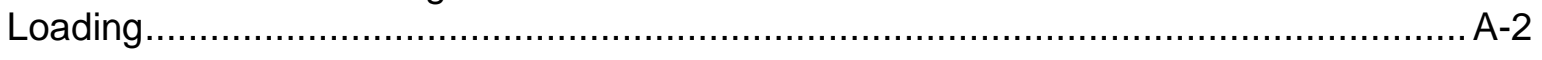

A.1.1.2 Simple Support Beam Theory and Curvature Moment Approach..................... A-4

A. 2 Impact of Boundary Conditions to Cable Bending Moment Evaluation ....................... A-6

$B$ MIXED-MODE LOADING CONDITION OF A TWISTED CONDUCTOR WIRE

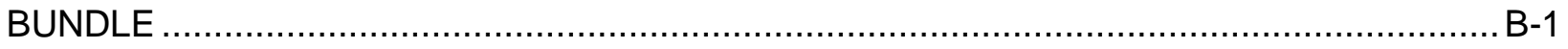

B.1 Combined Stress States of a Helical Wire Under Tension ......................................... B-1

B.2 Finite Element Analysis of Conductor Cable with Helical Twisted Wires Under

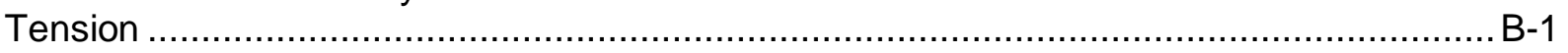

B.2.1 Single Straight Wire Vs. Single Twisted Wire .............................................. B-2

B.2.2 Conductor Cable with Bundles of Twisted Wires............................................ B-3 
B.2.3 Conclusion and Future Work on Mixed-mode Loading Analysis .................... B-7

C MECHANICAL TESTING RESULTS - CREEP, FATIGUE, AND THERMALMECHANICAL FATIGUE

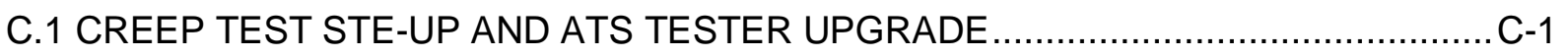

C 1.1 Creep and Fatigue test samples preparation ............................................

C.2 Creep Test Set-Up and Load Train Design ....................................................... C-2

C.3 Creep Testing Results for 1350-H19 Aluminum ................................................. C-6

C.2 Isothermal Fatigue Test Results...................................................................

C.3 Thermal-Mechanical Fatigue (TMF) Test Results.......................................... -34

DCONSIDERATIONS OF THERMAL CYCLING EXPERIMENTS FOR SINGLE STAGE

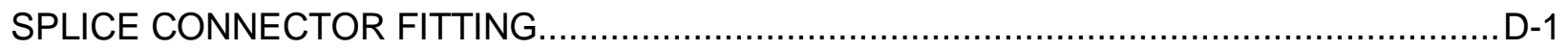

D.1 Issues Associated with SSC Thermal Cycling Experiments and the Conductor Aging 


\section{LIST OF FIGURES}

Figure 4-1 The parameters involved in HTLS lifetime estimates.

Figure 4-2 Thermal stress in AC current ........................................................................

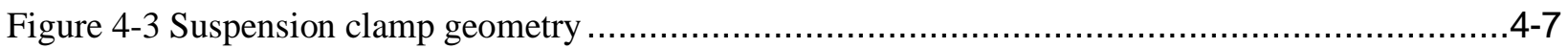

Figure 4-4 Schematic diagram at suspension site for bending moment evaluation ..........................4-8

Figure $4-5$ Helix geometry...........................................................................................

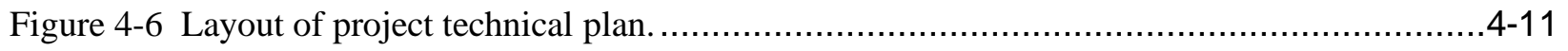

Figure 5-1 Detailed cross-section of a half ASCR SSC fitting....................................

Figure 5-2 Tensile and fatigue test set-up: environmental chamber, temperature and load controls and data acquisition system.

Figure 5-3 Close-up of environmental chamber and the extensometer, which is part of integrated test controls system for measurement deformation.

Figure 5-4 Close-up of the Laser Mike and the calibration band attached at the end of extensometer for strain measurement. .............................................................

Figure 5-5 High temperature tensile test with the associated deformation control set-up.

Figure 5-6 (a) Close-up of the dial gage used in the initial alignment of the tester loading train, and (b) set-up for fine tuning the tester alignment, where a dummy specimen equipped with strain gages is used to detect the stresses induced by the misalignment of the loading train. The associated control box is also shown in (b).

Figure 5-7 Set-up for calibrating the extensometer with the attached calibration band.

The calibration block is shown in the bottom left and the Laser Mike calibration

band is shown to the right at the end of extensometer.

Figure 5-8 ATS creep test machine's set-up with the environmental chamber and the control systems for heating, loading and deformation measurement.

Figure 5-9 A close-up of one ATS creep test machine, with a visual of the interior of the environmental chamber. The left tall cabinet is the furnace and temperature control system and the right cabinet is the load control system.

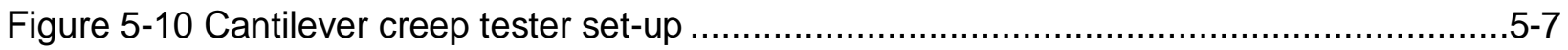

Figure 5-11 First order of 1350 aluminum shipped with an 8 inch diameter barrel.................5-7

Figure 5-12 Second order of 1350-H19 rod with \#1 AWG size, at 0.28 inch diameter. ............5-8

Figure 5-13 Pilot tensile testing set-up in an ATS creep machine with the associated

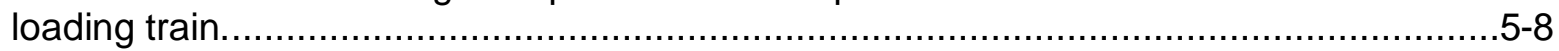

Figure 5-14 The end-grip design for pilot tensile testing. ........................................

Figure 5-15 The fractured sample of the pilot tensile testing for the first order of 1350-O........5-9

Figure 5-16 (Left) The typical slippage failure of 1350-H19 rods at grip-end during pilot tensile testing. The reduced section at the grip-end is due to a pipe fitting ring bitten 
into the testing sample. (Right) Details of a tensile sample failed at grip-end, and the intact side with the pipe fitting ring.

Figure 5-17 Tensile Specimen Design with 1 inch gage length and 0.18 inch gage diameter.

Figure 5-18 Fatigue Specimen Design with 0.5 inch gage length and 0.18 inch gage diameter.

Figure 5-19 End grip design for tensile and fatigue testing of the 1350- $\mathrm{H} 19$ rod.

Figure 5-20 Fractured tensile specimens and end-grip.

Figure 5-21 The tensile stress-strain curves for $1350-\mathrm{H} 19$ rod tested with $0.4 \% / \mathrm{min}$ strain rate loading.

Figure 5-22 Tensile test pairs done at $20^{\circ} \mathrm{C}, 120^{\circ} \mathrm{C}$, and $200^{\circ} \mathrm{C}$.

Figure 5-23 1350-H19 tensile tests done at 0.4\%/min, 4\%/min, and 8\%/min strain rate loading.

Figure 5-24 SSC fitting (left) and the associated core-grip (right)......

Figure 5-25 Crimped splice connector fittings: (a) TSC, and (b) SSC fitting; one SSC was cut into half that was used in neutron scattering experiments for residual stress measurements

Figure 5-26 Tensile and fatigue samples for 3003-H183 and 6061-T6 materials were machined directly from the baseline splice sleeve and the associated core-grip.

Figure 5-27 Tensile test results of 3003-H183 aluminum......

Figure 5-28 Comparison of tensile/compression trend curves for 3003- $\mathrm{H} 183$.

Figure 5-29 Comparison of tensile/compression trend curves for $1350-\mathrm{H} 19$.

Figure 5-30 Tensile test results of 6061-T6 aluminum

Figure 5-31 True stress vs. true plastic strain curves for (top) 1350-H19, (middle) 3003H183, and (bottom) 6061-T6 aluminums; Left column is the baseline data and the right column is the data from the annealed samples, with $260^{\circ} \mathrm{C}$ annealing temperature at a duration of 10 hours.

Figure 5-32 Creep test results for $1350-\mathrm{H} 19$ aluminum with gage diameter of 0.18 -in. For the creep test carried out at $70^{\circ} \mathrm{C}$, initial applied load was $60-\mathrm{lb}$ and after 8,000 min 180-lb was applied to the creep test.

Figure 5-33 Room temperature fatigue test with $0.2 \%$ strain cycle, the specimen failed after reaching 15,600 cycles.

Figure 5-34 Fatigue test carried out at $200^{\circ} \mathrm{C}$ with $0.25 \%$ strain cycle. The material shows pronounced softening and permanent deformation after about 150 cycles, and the sample material fails to carry stress after about 350 cycles.

Figure 5-35 1350H-19 TMF test with total strain maintained near 0\% and temperature cycle range of $75^{\circ} \mathrm{C}$ to $175^{\circ} \mathrm{C}$. The material softening and hardening appeared in alternative TMF cycles seem to balance each others and no permanent aging damage was observed.

Figure 5-36 $1350 \mathrm{H}-19 \mathrm{TMF}$ test with total strain cycling between $0 \%$ to $0.2 \%$ and temperature cycle range of $100^{\circ} \mathrm{C}$ to $150^{\circ} \mathrm{C}$. No significant aging was observed from the TMF data.

Figure 5-37 Fatigue fracture samples, with typical striation formation, yellow arrow indicates the orientation of the fatigue crack growth. 
Figure 5-38 SEM images of typical 1350-H19 fatigue fractured samples.

Figure 5-39 The impedance spectra from baseline and the aging splice connectors............5-25

Figure 6-1 Dimensions of 6012CD Round Die Half Used for Single-stage Fittings ........................6-3

Figure $6-2$ Geometry of the single stage compressive splice connector ..............................6-4

Figure 6-3 Detailed cross-section of a half single stage splice connector

Figure 6-4 Typical cross-sections of ACSR Drake SSC system: (left, middle) core-grip section, and (right) conductor section.

Figure 6-5 Free body diagrams of load transfer during rated breaking strength tensile test; (top) sleeve carries the full rated breaking strength at the middle section of the sleeve, the tensile stress distribution of the conductor and core steel within the SSC fitting are non-uniform as illustrated with two triangular blocks for conductor and core-grip sections, respectively; (bottom) shear force resistance at sleeve/conductor interfaces and core-grip/steel-core interface.

Figure 6-6 Finite element model (FEM) of an ACSR Drake SSC fitting (left) before forming, (right) after crimping

Figure 6-7 FEM models for core-grip section of Drake splice connector with 6012CD die: (top) before crimping and (bottom) crimped SCC.

Figure 6-8 von Mises effective stress fringes of FEM analysis for 3P (top) and 15D (bottom) core-grip positions: (left) at the die-closure; (right) after the removal of the die-set, which represents the effective residual stress field remained in the core-grip section.

Figure 6-9 von Mises effective stress fringes of FEM analysis for Vert core-grip position: (left) at the die-closure; (right) after the removal of the die-set.

Figure 6-10 2-D FEM forming simulation for conductor section: (left) un-deformed baseline, (right) crimped SCC at die closure.

Figure 6-11 Normal directions of the potential contact surfaces for aluminum conductor layers.

Figure 6-12 (Left) Un-deformed baseline SSC FEM model at conductor section, (right) crimped conductor section at the middle section of FEM model after the die-set removal.

Figure 6-13 FEM simulated deformed configuration for SSC fitting at the conductor section upon die-closure: (left) iso-view, (right) side-view.

Figure 6-14 von Mises stress contours at middle layer of a crimped SSC conductor section: (left) at die-set closure, (right) after die-set removal.

Figure 6-15 Neutron diffraction in a lattice structure.

Figure 6-16 Schematic diagram of neutron diffracted by the target material and using PSD detector to determine the peak angle, $\theta_{p k}$

Figure 6-17 Experimental set-up at NRSF2 facility for residual strain measurement of a crimped connector.

Figure 6-18 The direction of the radial residual strain profile, as indicated with red line, is about $120^{\circ}$ to the long axis of the deformed crimped sample.

Figure 6-19 Radial lattice spacing profile at core grip section.

Figure 6-20 Radial lattice spacing profile at the aluminum conductor section. 
Figure 6-21 Cross-sections of the irradiated SSC samples: (left) core-grip section, (right) conductor section.

Figure 6-22 Radial displacement at core-grip section along $120^{\circ}$, path from center steel core to the outer aluminum.

Figure 6-23 Residual elastic strain in core-grip section at $120^{\circ}$ and $130^{\circ}$, the X-axis is the deformed coordinate.

Figure 6-24 Residual stress in core-grip section at $120^{\circ}$ and $130^{\circ}$, the $X$-axis is the deformed coordinate.

Figure 6-25 Residual elastic strain in radial direction along the $120^{\circ}$ (blue line) and $130^{\circ}$ (purple line) neutron beam directions. $\mathrm{X}$-axis is the original undeformed mesh coordinate

Figure 6-26 Residual stress in radial direction along the $120^{\circ}$ (blue line) and $130^{\circ}$ (purple line) neutron beam directions. $X$-axis is the original undeformed mesh coordinate

Figure 6-27 Comparison of residual stress profiles at core-grip section

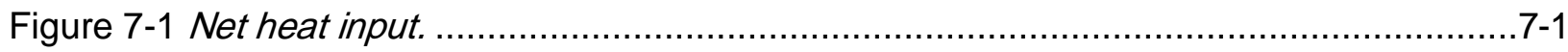

Figure 7-2 Schematic diagram of SSC system components and their functionalities..............7-1

Figure 7-3 Schematic diagram of SSC system interfaces........................................... $7-2$

Figure 7-4 Typical thermal cycles of 6 cycles per day with target temperature of $150^{\circ} \ldots \ldots \ldots \ldots .7-3$

Figure 7-5 Maximum temperatures at conductor/splice sleeve interface per cycle within ACSR SSCs measured from 10 thermocouples, with target conductor surface temperature of $150^{\circ} \mathrm{C}$ at 15 feet away from the SSC. Temperature increased dramatically in ACSR SSC system during thermal cycles....................................... $7-5$

Figure 7-6 Maximum cycle temperature history for quenched data. ................................. 7-5

Figure 7-7 Maximum cycle temperatures for non-quenched data..................................... $7-6$

Figure 7-8 Significant decrease in tension lading of the test spans that contains SSC

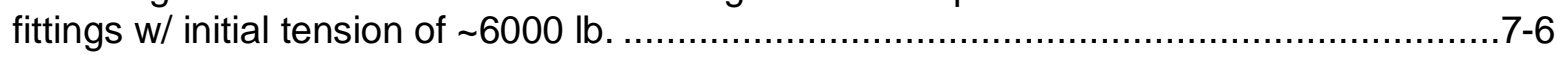

Figure 7-9 Temperature profile history of conductor section in SSC system used for FEM

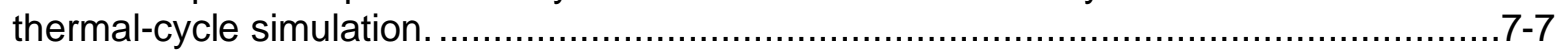

Figure 7-10 Temperature profile history of core-grip section in SSC system used for FEM

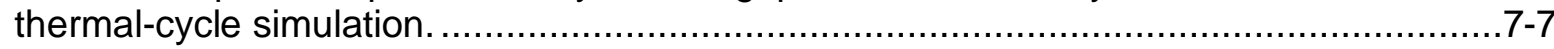

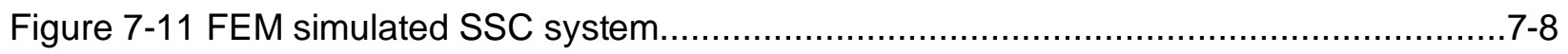

Figure $7-12 \mathrm{FEM}$ thermal cycle simulation (left) at $315^{\circ} \mathrm{C}$ cycle range no tension loading, (right) at $225^{\circ} \mathrm{C}$ cycle range with tension loading. .

Figure $7-13$ (left) without tension simulation at $315^{\circ} \mathrm{C}$ thermal cycling range, and (right) with tension at $275^{\circ} \mathrm{C}$ thermal cycling range.

Figure 7-14 FEM Simulation at $225^{\circ} \mathrm{C}$ with quench and tension loading applied. ..................7-10

Figure 7-15 The compressive force in conductor section at heat-up cycles......................... 7-11

Figure 7-16 Compressive force in conductor section at cool-down................................... 7-11

Figure 7-17 Enlarged section view of compressive force in conductor section at the first

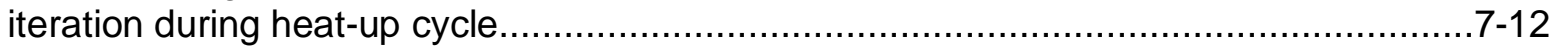

Figure 7-18 FEM model of SSC core-grip section center at steel core . ........................... $7-12$

Figure 7-19 Compressive force around steel-core at heat-up cycle................................ $7-13$ 
Figure 7-20 Compressive force around steel-core at cool-down.................................. $7-14$

Figure 7-21 Compressive force in conductor section at heat-up.................................. $7-15$

Figure $7-22$ Compressive force in conductor section at cool-down................................ $7-15$

Figure 7-23 Compressive force around steel-core at heat-up..................................... $7-16$

Figure 7-24 Compressive force around steel-core at cool-down.................................... 7-16

Figure 8-1 The temperature-dependent shear resistance trends for SSC Drake conductor system.

Figure 8-2 Sum of the shear resistance forces from the SSC conductor and core-grip sections.

Figure 8-3 The shear resistance capacity of ACSR Drake SSC system with 16-in long net core-grip and conductor section.

Figure 8-4 Drake conductor after SSC fitting pull-out test............................................ $8-5$

Figure 8-5 SSC fitting sleeve and the Drake conductor after the pull-out test.......................8-6

Figure 8-6 The shear resistance capacity for Drake SSC systems ..............................8

Figure 8-7 Drake SSC shear resistance capacity at high temperature ................................8-8

Figure 8-8 Maximum cycle temperature $\left({ }^{\circ} \mathrm{C}\right)$ at SSC splice surface. ................................ 8-10

Figure 8-9 The tested SSC spans tension-thermal cycle history; spans 3 and 4 were designated for water quenched test spans.

Figure 8-10 SSC surface temperature history obtained from thermal cycle experiments with $150^{\circ} \mathrm{C}$ conductor control temperature.

Figure 8-11 ASCR Drake SSC effective lifetime trend curves.

Figure 8-12 ACSR Drake SSC lifetime prediction for shear capacity < 6000lb.

Figure 9-1 Schematic diagram of the developed SSC effective lifetime estimate protocol 



\section{LIST OF TABLES}

Table 5-1 Summary of the material properties for the $1350-\mathrm{H} 19$ aluminum rod..................5-12

Table 5-2 Summary of $A B I$ test results ...................................................................

Table 6-1 Normal contact pressures between core-grip and steel of 15D position ................6-8

Table 7-1 Temperature profiles within SCC and TSC fittings........................................

Table $7-2$ Temperature profiles of SCC fitting ............................................................

Table $7-3$ Stress distribution for 600 -ft span Drake conductor ...................................... $7-8$ 


\section{1 eXeCUtIVE SUMmary}

Due to the increase in power demand and limited investment in new infrastructure, existing overhead power transmission lines often need to operate at temperatures higher than those used for the original design criteria. This has led to the accelerated aging and degradation of splice connectors, which have been manifested by the formation of hot-spots that have been revealed by infrared imaging during inspection. The implications of connector aging is two-fold: (1) significant increase in resistivity of the splice connector (i.e., less efficient transmission of electricity) and (2) significant reduction in the connector clamping strength, which could ultimately result in separation of the power transmission line at the joint. Therefore, the splice connector appears to be the weakest link in the electric power transmission lines. This report presents a protocol for integrating analytical and experimental approaches to evaluate the integrity of a full tension single-stage splice connector assembly.

This research project has developed a methodology for evaluating splice connector system performance and the associated effective lifetime estimate, under high temperature operation. In particular, the case of aluminum conductor steel reinforced (ACSR) conductors was addressed. The implementation of the proposed research allows electric utilities to predict the service life of splice conductor systems for any arbitrary loading history that includes geographic location and number of thermal cycles associated with operation during peak periods. The research effort focuses on the long-term impacts of conductor life due to thermal cycle effect under high operation temperature.

The research activities involve the following efforts:

- Thermomechanical evaluation and physical characterization of aluminum alloys used for the fabrication of a SSC system of the ACSR conductors. The thermomechanical tests include uniaxial tensile tests to determine the stress-strain behavior of the alloys at various temperatures, creep stress-rupture and fatigue tests, and the thermal-mechanical fatigue tests. Physical characterization includes microstructural characterization and determination of physical properties, such as temperature dependent friction coefficient.

- $\quad$ Development of time-dependent failure criteria and experimentally-based mechanical property models, which are the basis of an inelastic design analysis.

- Incorporation of models into computational tools for parametric studies, predictions and analyses of SSC thermal cycling tests. A finite-element program that models the stresses, strains and the associated geometry deformation in the vicinity of the splice-conductor interface region, coupled with the constitutive models was used for this purpose.

- Confirmatory structural verification evaluation, compared the simulation results with those of the neutron scattering experiments and the thermal cycling experiments.

This research addresses the needs of electric utilities because it provides them with tools for improving the reliability of the electrical grid through the formulation of time-dependent failure 
criteria that will assure adequate service life of ACSR SSC system. These tools will aid electric utilities in making decisions regarding maintenance of the grid and response to power demands.

The developed governing equation that can be used for predicting the SSC effective lifetime is described below.

$$
\begin{aligned}
& R_{S R}=20992 * \exp \left(-0.024 * 0.6375^{*} T_{\text {cond }} * N_{f}^{0.0969}\right) * L_{\text {cond }}+ \\
& 14449 * \exp \left(-0.02 * 0.6375^{*} T_{\text {cond }} * N_{f}^{0.0969}\right) * * L_{\text {core }}
\end{aligned}
$$

where, $\boldsymbol{R}_{S R}$ is the effective shear resistance capacity provided by SSC system; $L_{\text {cond }}$ and $\boldsymbol{L}_{\text {core }}$ are the effective length of the conductor and core-grip sections, respectively; $T_{\text {cond }}$ is the conductor operating temperature; and $\boldsymbol{N}_{\boldsymbol{f}}$ is the number of the thermal cycling during the service. 


\section{GLOSSARY OF TERMS AND DEFINITIONS}

ACSR - Aluminium Conductor Steel Reinforced.

ACSS - Aluminium Conductor Steel Supported - A stranded conductor made up of fully annealed aluminium strands over a core of steel strands.

Ampacity - The ampacity of a conductor is that maximum constant current which will meet the design, security and safety criteria of a particular line on which the conductor is used. In this brochure, ampacity has the same meaning as "steady-state thermal rating."

Annealing - The process wherein the tensile strength of copper or aluminium wires is reduced at sustained high temperatures.

ASTM - American Society for Testing and Materials.

Electrical Clearance - The distance between energised conductors and other conductors, buildings, and earth. Minimum clearances are usually specified by regulations.

Knee-point Temperature - The conductor temperature above which the aluminium strands of an ACSR conductor have no tension or go into compression.

Maximum Allowable Conductor Temperature - The highest conductor temperature at which an overhead power line can be safely operated.

RBS - Rated Breaking Strength of conductor. A calculated value of composite tensile strength, which indicates the minimum test value for stranded bare conductor. Similar terms include Ultimate Tensile Strength (UTS) and Calculated Breaking Load (CBL).

Thermal Rating - The maximum electrical current, which can be safely carried in overhead transmission line (same meaning as ampacity).

Uprating - The process by which the thermal rating of an overhead power line is increased.

"Worst-case” weather conditions for line rating calculation - Weather conditions which yield the maximum or near maximum value of conductor temperature for a given line current.

HFIR - the high flux isotope reactor at Oak Ridge National Laboratory.

TMF - thermal mechanical cycle fatigue.

FEM - finite element method. 
$\boldsymbol{F}$ - the governing equation of temperature-dependent shear resistance force, and the subscripts "cond' and "core" stand for conductor and core-grip sections, respectively.

$L$ - the effective length, and the subscripts "cond" and "core" stand for conductor and core-grip sections, respectively.

$T$ - the max. cycle temperature at SSC splice surface.

$T_{\text {cond }^{-}}$the conductor operating temperature.

$\boldsymbol{N}_{\boldsymbol{f}}$ - the number of thermal cycles.

$\boldsymbol{R}_{\boldsymbol{h}}$ and $\boldsymbol{R}_{\boldsymbol{C}}$ - the shear resistance capacities at heat-up and cool-down cycles, respectively.

$\boldsymbol{R}_{S R^{-}}$the effective shear resistance capacity provided by SSC system.

SSC - the single stage splice connector fitting. 


\section{INTRODUCTION}

One of the primary questions regarding the use of HTLS conductors concerns their anticipated service life. That is, how long will they continue to perform satisfactorily. Upon reflection, the research team decided that this question was difficult to answer, even for a conventional conductor, let alone HTLS. Therefore, it was decided to study the service life of conventional steel-reinforced core aluminum conductors (ACSR) before trying to determine this for HTLS. When considering the service life of ACSR, it became apparent that the connectors and terminations of ACSR, especially single stage connectors and terminations, were the least robust component in the conventional conductor system. Therefore, an attempt has been made to determine the service life of single stage compression splices for ACSR.

The majority of overhead transmission lines currently in use are ACSR. ACSRs were designed to operate at temperatures near $100^{\circ} \mathrm{C}$ and, for limited periods of time (e.g.during emergencies) at temperatures as high as $125^{\circ} \mathrm{C}$. These temperature limits constrain the thermal rating of a typical 230-kV line to about 400 MVA. Crimped-type splice connectors play an important role in the efficiency and reliability of power transmission systems. Failure of connectors, and thus transmission lines, are often attributed to errors in installation or on the use of inadequate materials. For example, failure occurs when crimped-type splice connector fittings do not have adequate crimping pressure or when the conductor is clamped over a length that is shorter than that prescribed for installation. Utilities face critical decisions about the management and service life of transmission lines as a result of increasing power demands, including the operation of transmission lines at higher temperatures. As a result, the rate of transmission line failure will likely increase. Furthermore, annealed strands will be more prone to creep deformation, which would lead to relaxation of connector clamping stress and eventually to the pullout of the conductor. This situation is further worsened with thermal expansion/contraction cycles as a result of variations in the conductor loading. Moreover, the joint's electrical resistance will increase as a result of oxide layer built-up, corrosion processes, inter-diffusion, electromigration, and fretting. All this leads to reductions in the efficiency of the system.

\subsection{High Temperature Low Sag Conductor (HTLS) Lifetime Prediction Program}

EPRI and DOE are jointly sponsoring research at Oak Ridge National Laboratory to develop an inelastic design methodology for HTLS conductors and to formulate time-dependent failure criteria to assure their adequate service life. The goals of this project are to provide accurate estimates of power line performance and lifetime, and to provide a basis for developing industry guidelines, and consensus standards. The potential benefits and outcomes of this HTLS Lifetime Program are:

- Optimize existing power grids

- Prevent conductor system damage

- Accelerate decision making processes 
- Reduce maintenance cost \& outages

This research project has been focused on developing lifetime estimates for single-stage splice connectors (SSC), which are considered to be a limiting factor in current power transmission systems. To gain a clear understanding of SSC design and functionality, a unique computer simulation protocol has been developed to identify potential failure mechanisms and to evaluate the long term reliability of SSCs.

The work performed as part of the proposed research includes the following tasks.

Task 1) Thermomechanical evaluation and physical characterization of aluminum alloys used for the fabrication of SSC system components.

Task 2) Development of time-dependent failure criteria and experimentally-based inelastic constitutive models.

Task 3) Incorporation of models into computational tools for parametric studies with predictions of remaining life and analyses of structural tests.

Task 4) Confirmatory structural verification evaluation.

These tasks are intimately related to each other, and the ultimate objective of this project, namely, predicting the service life and remaining strength (or effective lifetime) of SSC conductor system, could not be achieved without all the tasks being performed as part of one project. 


\section{BACKGROUND}

The long-term goal of this research is to develop an inelastic design methodology for HTLS conductors and the associated connector system to formulate time-dependent failure criteria that will assure their adequate service life. The implementation of the results from the proposed research will allow electric utilities to predict the service life of HTLS conductor systems for any arbitrary loading history that includes geographic location, tensioning load during erection and the number of thermal cycles associated with operation during peak periods. The implementation of HTLS conductors and a methodology for predicting their service life could result in significant improvements in the transmission capacity of the electric grid and in its reliability, without significant modifications to its infrastructure. A detailed listing of references associated with the subjects of the high temperature operation of power transmission lines are provided in the Reference section.

\subsection{Background of Thermo-mechanical Loadings in HTLS Conductors.}

The use of new HTLS conductors will require the implementation of new rules for their qualification and testing. In general, materials and components respond inelastically to thermal and mechanical loadings at elevated temperatures. At the lower end of a material's useful elevated-temperature operating range, the inelastic response can usually be separated into timeindependent plasticity and time-dependent creep. Each material can exhibit complex, historydependent hardening or softening, and the two types of response can interact with one another; i.e., prior plastic strains affect subsequent creep response, and vice versa. At higher temperatures, the distinction between rate-dependent plasticity and time-dependent creep blurs for many materials and the separation between behaviors is no longer valid. The response becomes very rate dependent and both strain- and cyclic softening occur. Thus, a well designed testing procedure is needed to systematically determine the evolution of the stated variables through experimentation. Moreover, regarding the interaction of fatigue, oxidation and creep damage, a "unified creep-plasticity constitutive model" is considered. These models do not separate plastic and creep strain components, but treat them in a unified manner, such as inelastic strain. Unified theories are based on a state representation (material state) that includehardening and recovery functions consistent with the Bailey-Orwan theory. These theories have the potential to predict material response for creep and cyclic plasticity, and also time-dependent changes under isothermal and thermo-mechanical loading- once the constitutive equations and material constants are established.

In order to carry out a realistic experimental study, we need to understand the current practice of power line installation. The following steps need to be considered.

1. Determination of the baseline material properties as a function of temperature. The temperature range must be representative of actual service conditions.

2. Determination of the sag-limit tension loading and other relevant dynamic loads.

3. Determination of the stress-strain behavior of the conductor as whole and of the individual strand's per sag tension loading. 
4. Determination of steady-state thermal rating: Estimates will be obtained for the maximum allowable temperature and ranges based on the different heat sources or sinks as illustrated below. EPRI's database will be used as reference source.

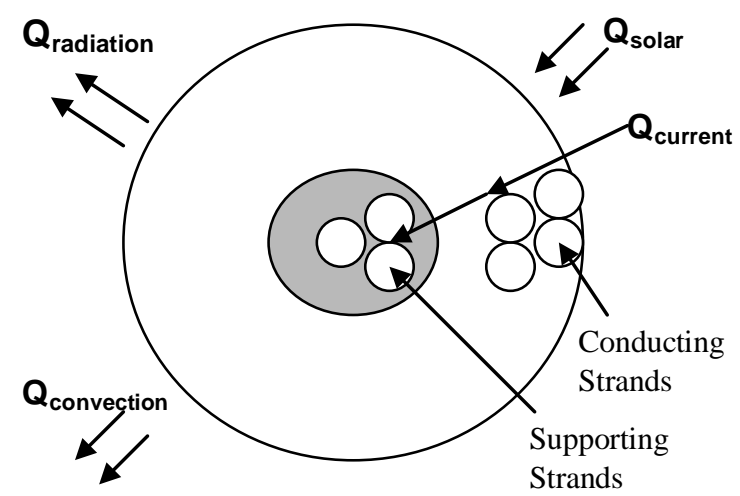

5. Assessment of the net effect of creep produced by various temperature levels for certain time intervals. The time-dependent fatigue criteria and the associated environmentally induced effects will be investigated.

6. Assessment of wind induced conductor motion:the Aeolian vibration and ice galloping effects.

7. Analysis of Voltage uprating of existing lines. This determines the maximum allowable operating temperature based on the available (or aging model from this study) prediction index for aging power line material characterizations.

In general, engineering structures are subjected to primary and secondary stresses. In the case of HTLS conductors, primary stresses are associated with tensile stresses resulting from line tensioning during erection, and bending and shear stress resulting from the conductor's weight. In addition to primary stress are secondary stresses, which result from geometric discontinuities or thermal gradients, such as those that exist at the interface between a conductor and a splice, or stresses that are random in nature, such as those resulting from aeolian vibrations and ice loading. In the case of HTLS conductors, most secondary stresses are cyclic in nature. For example, the temperature of the conductor will change due to variations in ambient conditions during the day and throughout the year, as well as other variations. For example, changes in the magnitude of the electric current flowing through the conductor during periods of peak demand.

Each time secondary stresses are cycled, the stress-strain state at each location in the conductor and splice undergoes a complex cycle involving plasticity and creepAt higher temperatures, time-dependent viscoplastic behavior are experienced. Therefore, the stress state during normal operation bears little resemblance to that envisaged for the primary stress due to line tensioning alone. Furthermore, the accumulated cyclic strains can be considerably larger than those due to creep alone. This latter phenomenon is referred to as inelastic ratcheting. Creep damage, creepfatigue damage (the synergistic interaction of time-dependent and cyclic damage), and ratcheting (excessive deformation) are the key failure modes of concern for HTLS conductors particularly at regions of geometric discontinuity, such as at the interface between a splice and the conductor.

To predict the remaining life of HTLS conductors it will be necessary to develop and apply models that account for both primary and secondary stresses and their interactions throughout the life of the conductor. The accuracy of those predictions will depend on how well the entire service history (stress, temperature and environment) experienced by the conductor is known, 
from the moment it was installed up to the moment for which the prediction is made. The schematic diagram of parameters involved in estimating the HTLS lifetime or aging model is illustrated in Figure 4-1.

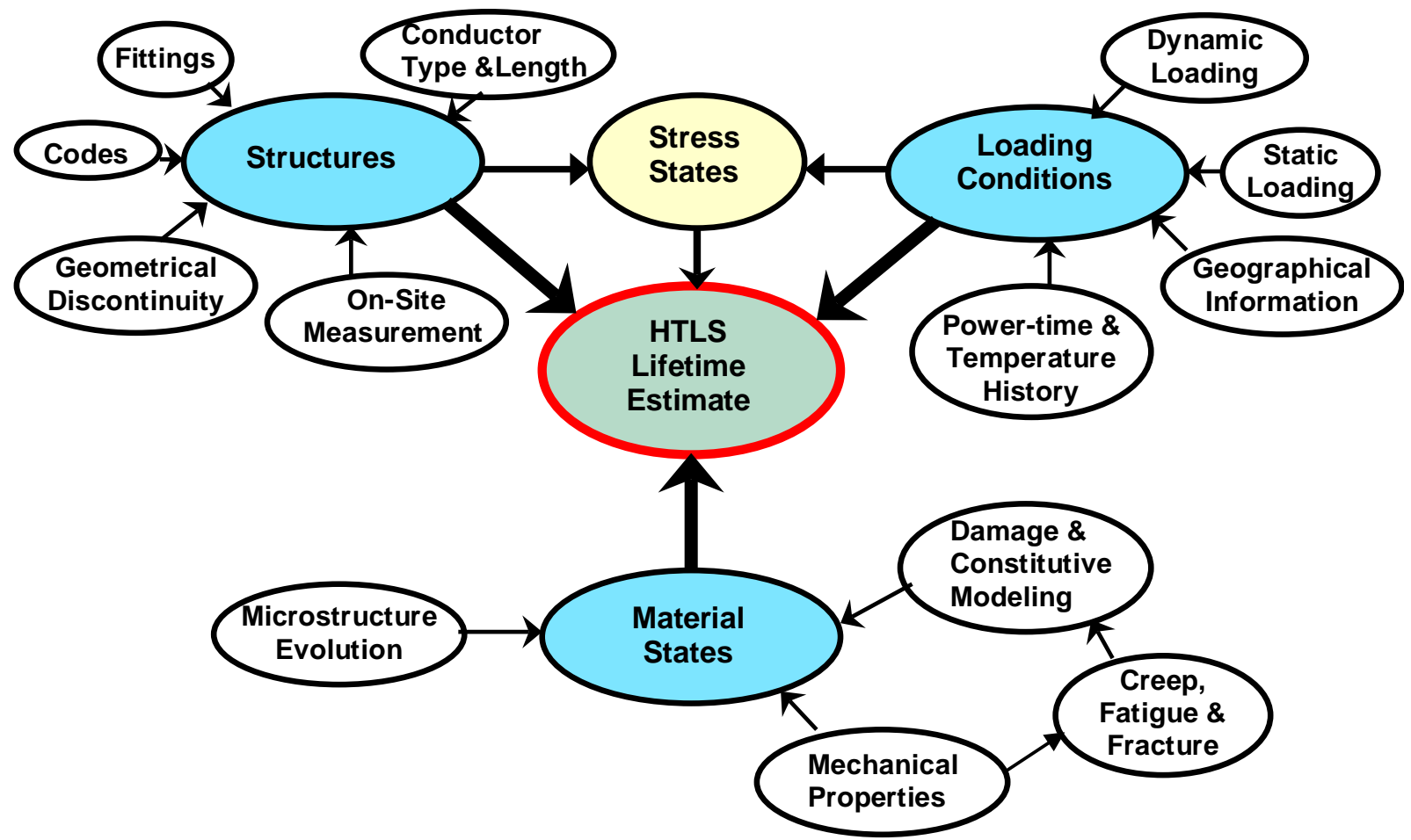

Figure 4-1 The parameters involved in HTLS lifetime estimates.

A model for predicting the remaining life of HTLS conductors must be capable of describing the evolution of deformation and damage in the conductor for any arbitrary loading history. The description of how deformation and damage evolve must occur at two different levels. First, the model should be capable of describing the time-evolution of deformation and damage in a volume of material (i.e. - the reinforcing core or the conductive phase) when subjected to spatially uniform stresses. Second, the model should be capable of describing how deformation and damage evolve in regions of components where stresses and strains are not uniform, for example, at the interface between the conductor and a splice, as in the case of HTLS conductors. Because the states of stress, deformation and damage in such regions are very complicated, the most effective approach to describe them requires the combination of constitutive inelastic models of conductor constituents (core, conductive phase and splice) and finite-element modeling techniques to account for the geometry of the conductor, including regions where stresses, strain and damage are concentrated. 


\subsection{The Impact of Material Aging and Microstructural Evolution to Lifetime Estimate of HTLS Conductors}

\subsubsection{Joint interfaces between splice and conductor}

Compression fittings come in several styles developed over years. They have different characteristics that will be influenced by elevated temperature operation. By standard, they are required to carry 95\% of the Rated Breaking Strength (RBS) of the conductor to be a "full tension” connector. Inhibitors made for compression fittings breakdown at $162^{\circ} \mathrm{C}$. Joint interfaces are likely to degrade after several operations above $93^{\circ} \mathrm{C}$ due to evaporation and boiling of the joint compound in the joint. (Especially important for consideration of existing joints to be operated above $90^{\circ} \mathrm{C}$.) Because the radial thermal gradient in a conductor or component can be significant, the internal temperature may be significantly higher. Connectors with high temperature and high current operations create added mechanical, electrical and thermal stresses on fittings, which can impact the performance and service-life of the component.

\subsubsection{Aluminum anodizing and its relation to the splice connector}

Anodizing aluminum is an electrochemical process that produces a clear oxide layer on the surface of metal. This oxide layer is extremely porous and hard. The pore structure when viewed under a high-powered microscope looks like a honeycomb or metal sponge. The formulation and thickness of the oxide scale (layer) varies, but is basically controlled by time, temperature, and current density. Anodizing insulates the surface and makes it non- or less-conductive. The formed alumina scale on the surface of the aluminum conductor wires or splice connector components will inevitably reduce the electrical and thermal conductivity of the aluminum wire and the associated connector component, thus increasing the operating temperature.

With continued operation at elevated levels, an unfortunate cycle is formed. First there is the path with lowest resistance path, this path becomes overused and develops into a path of increased resistance leading to more restictions and ever increasing resistance. With each cycle, the higher resistance generates greater heat (and therefore greater resistance) until the temperature gets so great, the connector softens and fails. The additional electrical resistance is also created by a "loosening" of the connection at interfaces between the conductor and splice fitting. This is due to the expansion and contraction of the conductor and fittings with the varying current cycles and climatic influences on the conductor system. Thus detailed knowledge of aluminum anodizing within a splice connector is critical to form an accurate estimate of lifetime for HTLS conductor near splice connector regions.

\subsubsection{Issues regarding fatigue damage associated with AC current}

A schematic diagram of the AC current induced thermal stress field within an AC transmission power line is shown in Figure 4-2. From Figure 4-2, the AC current will induce the temperaturetime history variation within a conductor due to the associated variation of the electric currenttime history. The consequence of the temperature-time history variation is the temperature induced thermal stress variation with time. The stress-time history shown in Figure 4-2 clearly indicates that the AC current induced sine form thermal stress field will provide a source of cycle fatigue contributing to conductor aging; such thermal cycle fatigue will be magnified by higher current or peak load conditions. As for DC power lines, due to relatively constant current vs. 
time histogram, the thermal cycle fatigue associated with the Alternate Current power line will not occur in DC environment.
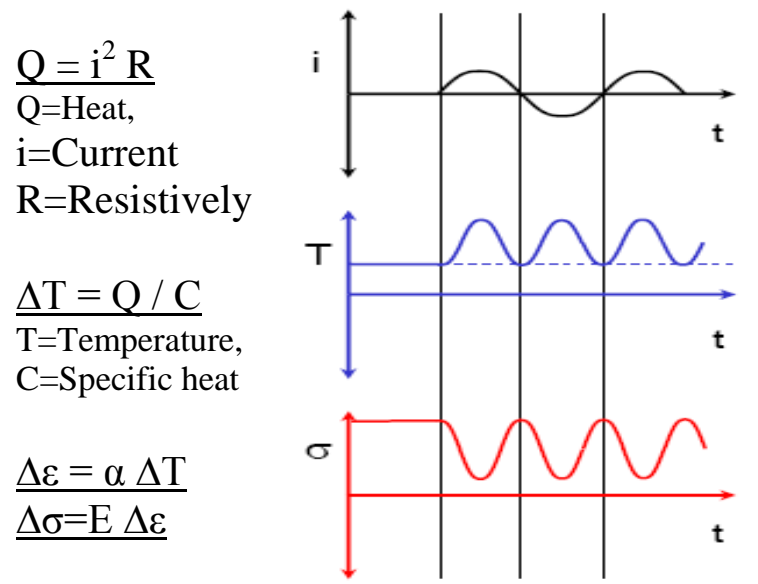

Figure 4-2 Thermal stress in AC current

\subsubsection{Electromigration microstructural damage of the conductor}

From the results observed from the in situ SEM electromigration experiments on $\mathrm{Al}$ interconnects (Al, Si and others), one may conclude that these tests can help us understand electromigration damage. It was found from the correlation of in situ damage micrographs with orientation mapping of the initial state that the void formation preferably occurs at the end of a blocking grain in polygranular segments. This is because of the interrupted diffusion path in current direction. However, because of the large number of blocking grains other reasons must exist for damage formation at certain places. Additionally, grain boundaries are necessary to exhibit a considerable misorientation of neighboring grains and run almost exactly in direction of current flow. Because of large misorientation, these boundaries possess high diffusivity which leads to increased material transport. Indeed, these phenomena are observed from a small scale interconnect device; however, it provides clues for further investigation of microstructural evolution and the associated lifetime estimates of the aging aluminum conductors. This high diffusivity phenomenon may have critical impact on material properties near the joint interface of splice and conductor. The diffusivity induced vacancy cluster may further reduce the electrical and thermal conductivity of the aging conductor.

\subsubsection{Flaw-induced localized high temperature spots}

It is known that an electrical current passing through a plate of material containing a defect (such as porosity, cracks, or inclusions) is concentrated at the tips of the defect. The induced high current density in conducting materials containing flaws (electric field concentrators) will load the defect faces with electromagnetic forces This tends to open them,due to current flowing in opposite directions along the defect faces. In addition, the tips of the defect act like single turn solenoid coils, which concentrate the magnetic flux and produce localized Lorentz forces along with local thermal stresses from Joule heating. The effective current density at the defect tips may be higher than all of the surrounding materials by one order of magnitude (depending on stress or field intensity factor). If the current density is high enough, the generated heat may be 
sufficient to melt the material at the defect tips, a phenomenon that has also been used to arrest fast-growing cracks. Nevertheless, these phenomena may further accelerate conductor aging and the increase the intensity of localized plastic deformation (or flow) on aluminum conductor materials, such as near the splice region or other geometrically discontinuous sites that have high a probability of forming flaws and defects. The potential for such damage mechanisms and the associated safety factor needs to be evaluated.

\subsection{The Impact of Bending Moment Effect to the Integrity of ACSR Conductor at Suspension Ends during High Temperature Operation}

The major difference between the beam and cable structures is the associated rigidity, where a beam has much larger bending and torsion rigidity (determined by its moment of inertia "I" and torsional inertia "J") than that of a cable. In a cable, the majority of the deformation strain energy results from tensile loading, but "bending loadings" also have a small portion to contribute. The relative rigidity (EI or EI/L) of a cable or a wire is likely to scale with its tension loading. The high tension loading will increase the relative rigidity of a cable or a wire. For example, it is much more difficult to deflect (or bend) a high tension loaded piano wire as compared to a low tension loaded piano wire. During erection of a conductor cable, without proper pretension, the erection induced bending moment can significantly damage the conductor cable. Therefore, pre-tension is necessary and required during the erection of a conductor cable.

In reality, a cable under a high tension loading reveals a much small bending moment as compared to that of a simple support beam with no axial tensile loading at the beam. This is due to the counterbalance of the moment derived from the cable dead load and the cable tensile loading. In general, most analyses of cable structures neglect the contribution from the bending moment when a suspended cable is subjected to tensile loading. However, due to the very small moment of inertia of a cable structure, a very slightly increase in the magnitude of the bending moment will significantly increase the magnitude of the flexural normal stress experienced by the outer layer of the cable. At high operating temperatures, the aluminum wires are all relaxed (in addition to annealing or bird-caging phenomena), and the steel core will likely carry all the bending moment, at the middle span or at a cable continuing-suspension end. For a Drake conductor, the steel core has 0.00092-in ${ }^{4}$ of moment inertia, $\mathrm{I}_{\mathrm{ST}}$, and the outmost radius of steel core is 0.2040 -in. At high temperatures, with every increase of $1 \mathrm{ft}$-lb bending moment, there will be a resultant increase of 2.7-ksi flexural normal stress at the outmost layer of the steel-core. ACSR steel core (ASTM-B498) wire has about 170-ksi strength, the yield stress is about 150-ksi. It will need a $56 \mathrm{ft}-\mathrm{lb}$ bending moment to yield the outer layer of the steel core when the conductor cable is at a high temperature operation.

A detailed cable bending moment investigation was carried out and is described in Appendix A. Three approaches were utilized for evaluating the cable bending moment, namely, simplified beam theory, tie-rod theory, and cable curvature theory approaches. These three approaches revealed similar results for different loading and conductor spans. The parabolic geometry of a conductor cable was used in the cable bending moment analyses. All three approaches show small bending moments for a simple support span of a Drake conductor cable. Therefore, it is expected that during the conductor service life the bending moment there will be less impact on the conductor near mid-span (the maximum bending moment site of a simple support span). However, this may not be the case for a cable suspension end of a continuing span; furthermore the typical catenary or parabolic theory of the conductor cable is not applicable. The localized 
cable curvature at the suspension location will dictate the effective bending moment of the conductor cable. This scenario will be further investigated in the following section.

Extending the theoretical formulation, curvature or sag evaluation, to a high temperature environment, where the service material may experience a large plastic deformation or thermal annealing, is very questionable. The issue regarding high temperature effects on sag evaluation was discussed in literature published by a Canadian utility in 1980. It mentions that W.P. Dobson Research Laboratory carried out research evaluating the high temperature sag data on a number of conductors. These test spans were $122-\mathrm{m}$ and $366 \mathrm{~m}$ in length and a total of six conductors were tested at this facility in the $-10^{\circ} \mathrm{C}$ to $350^{\circ} \mathrm{C}$ temperature range. In all cases, the measured sags at higher temperature were found to be considerably larger than those calculated by accepted methods. The direct consequent of higher sag is the increase of the curvature at the mid-span and at the cable suspension ends.

\subsubsection{Cable Continues Through the Suspension Ends}

As discussed earlier, for a conductor continuing through the suspension ends, the bending moment at the suspension ends will be dictated by the geometric configuration of the suspension clamp (such as initial slope of the conductor at the suspension-clamp end) as shown in Figure 4-3. Furthermore, the localized curvature variation cycles are also strongly dependent on thermal cycling events. The potential of fatigue damage at the suspension ends due to the repeated alternation of bending moment caused by the thermal cycling event is likely, especially at high operating temperature. A schematic diagram for the bending moment evaluation at the suspension site is shown in Figure 4-4.

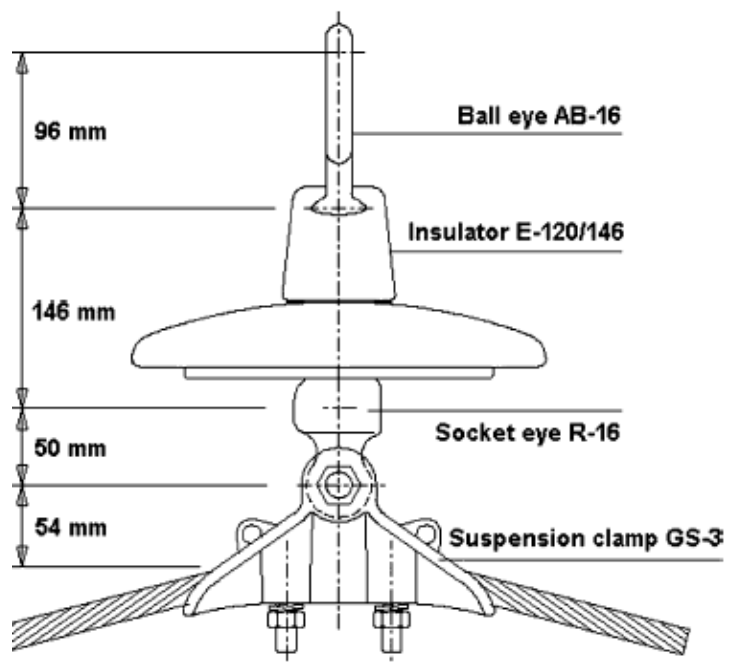

Figure 4-3 Suspension clamp geometry 


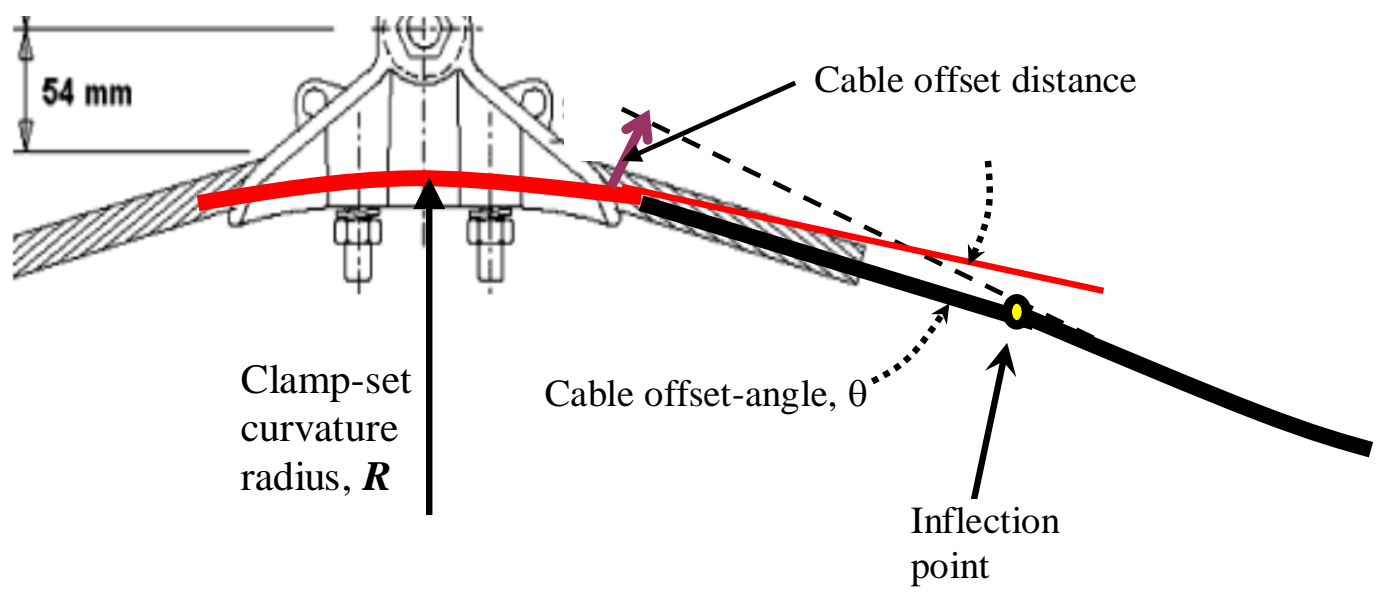

Figure 4-4 Schematic diagram at suspension site for bending moment evaluation

The methodology used to evaluate the maximum bending moment at the cable suspension site is described below.

(1) Based on the bending moment curvature theory, the initial bending moment of the conductor cable at the clamp site is equal to $\mathrm{EI} / \mathrm{R}$, where the $\mathrm{R}$ is the curvature radius of the clamp-set, as shown in Figure 4-4.

(2) The additional bending moment is due to cable tensile loading and the cable offset to the clamp-set, as described below.

Moment $=$ Cable tension $(\mathrm{T}) *$ Cable-offset distance $\left(\mathrm{L}_{\text {off-set }}\right)$

Where the cable offset distance is shown in Figure 4-4

(3) Thus, the overall bending moment at the suspension site from the static tensile loading is

$$
\text { Moment }=\mathrm{EI} / \mathrm{R}+\mathrm{T}^{*} \mathrm{~L}_{\text {off-set }}
$$

For a 3000-lb tension loading and a 1/4-inch cable-offset, the bending moment contributed by the cable tension is equal to $62.5-\mathrm{ft}-\mathrm{lb}$. As indicated earlier, it only requires $56-\mathrm{ft}-\mathrm{lb}$ to yield the outer layer steel-core at high operating temperature. Therefore, a $1 / 4$-inch offset of the conductor cable to the end of the clamp-set, tensile loading induced bending moment will start to yield the steel-core wires and results in permanent deformation of the steel core at the suspension ends of a continuing span. Thermal cycling induced cable geometric changes, such as variations of cable offset at the suspension ends, will also introduce fatigue damage to steel-core wires associated with variation of bending moment cycles, at high operating temperatures.

\subsubsection{Potential bending moment induced damage at suspension ends during high temperature operation}

1350-H19 aluminum conductor wire of a Drake conductor at room temperature has a tensile strength of $24 \mathrm{ksi}$ and yield stress of 18 20 ksi. Below the knee point, the aluminum conductor is still within linear elastic range and will carry most of the bending moment for the cable due to the relatively large moment of inertia of $0.0532-\mathrm{in}^{4}$, which is about 58 times higher than that of the steel-core. But above $212^{\circ} \mathrm{F}$, the knee point, the aluminum conductor strands will relax and 
potentially have bird-caging near the suspension ends or at the constraint sitesIn addition a significant plastic flow is expected at higher temperatures. Furthermore, the yield stress of the conductor wire will be reduced significantly at relatively higher temperatures. Therefore, steelcore wires are expected to carry the most bending moment load, such as at the cable suspension ends of a continuing span. Since sagging will continue to increase with increasing temperature (but with less intensity as it passes the knee point) and the bending moment will likely continue to increase at the suspension site of a continuing span. At high temperatures the bending moment resistant capability of the aluminum wires reduce significantly, thus the cyclic thermal loading will further result in accelerated aging of the steel-core wires and eventually may cause steel core failure.

This preliminary study may indicate that the effect of the bending moment on the integrity of the conductor lifetime deserves special attention. This study also indicated that the aluminum conductor is acting as a structural component in respect to "carrying bending moment" point.

\subsection{Mixed-mode Loadings Condition of a Twisted Conductor Wire Bundle}

A mixed-mode loading condition, tensile and flexural normal stress plus shear stress, will occur within a helical wire under tension loading along the central axis of the helical wire. For demonstration purposes, one assumes that the wire diameter is much less than the radius of the helix. The geometry of a helical wire is illustrated in Figure 4-5. At any point on a helical wire, point $\mathbf{A}$, the tangent to the helical center line of the wire is not parallel to the tensile loading $\mathrm{P}$. Therefore, the tensile force $\mathrm{P}$ will produce a bending moment (about the axis $\mathbf{n} \mathbf{1}$ ) and a torque at the cross section of $\mathbf{A}$ on a helical wire, and tensile loading at $\mathrm{A}$. The force $\mathrm{P}$ is resolved into two components: $\mathrm{P}^{*} \cos \theta$, perpendicular to the tangent of helical wire at $\mathbf{A}$, and $\mathrm{P}^{*} \sin \theta$, parallel to the tangent of the helical wire at $\mathbf{A}$. At the cross section of point $\mathrm{A}$ the component $\mathrm{P}^{*} \cos \theta$ produces the torque $\mathrm{T}=$ $\mathrm{P}^{*} \mathrm{R}^{*} \cos \theta$, where $\mathrm{R}$ is the radius of the helix, and the component $\mathrm{P} \sin \theta$ produces the bending moment $\mathrm{M}=\mathrm{P} * \mathrm{R} * \cos \theta$.

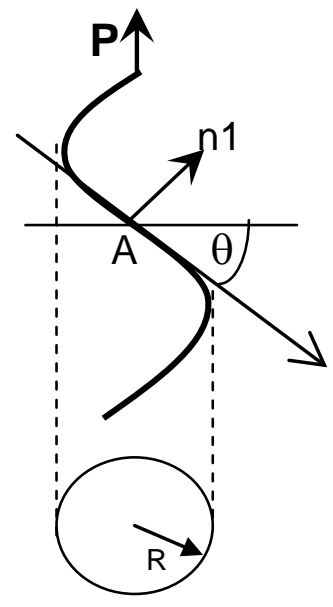

Figure 4-5 Helix geometry.

The maximum combined stress is $\sigma_{\max }=16\left(\mathrm{M}+\sqrt{ }\left(\mathrm{M}^{2}+\mathrm{T}^{2}\right)\right) / \pi \mathrm{d} 3=16 \mathrm{PR}(1+\sin \theta) / \pi \mathrm{d} 3$, where $\mathrm{d}$ is the diameter of the wire. The maximum shear is $\tau_{\max }=16\left(\sqrt{ }\left(\mathrm{M}^{2}+\mathrm{T}^{2}\right)\right) / \pi \mathrm{d} 3=16 \mathrm{PR} / \pi \mathrm{d} 3$. This exercise may indicate that the outer layer of the conductor cable has relative larger helix radius and will experience larger normal and shear stresses than that of inner layer of the conductor cable. However, the magnitude of these stress fields is expected to be significantly reduced in a conductor cable as compared to that of a single helical wire. This is because the lateral support to the helical wire was received from the adjacent twisted conductor wires, thus reducing the associated bending moment and torque induced by the helical wire geometry. This condition was further investigated with a more detailed finite element analysis as illustrated in Appendix B. 


\subsection{Approach}

Increasing demand on the electric grid has resulted in utilities investigating the use of (HTLS) conductors to increase the capacity of the transmission system without having to modify the existing structures. However the use of new HTLS conductors will require the implementation of new requirements for their design, qualification, and models to predict their service life.

The proposed research aims to investigate the time-dependent failure modes and time- and ratedependent deformation responses of HTLS conductors and their materials to time-varying thermal and mechanical loadings. The tasks envisioned in this research project will provide the data and models required to formulate time-dependent failure criteriaIt can also be used to predict remaining life of HTLS conductors for an arbitrary set of mechanical and thermal loading and environmental conditions. The envisioned tasks will also provide tools, based on an inelastic design analysis, to assure adequate life of HTLS conductors.

\subsubsection{Research Tasks}

The performed tasks are intimately related to each other, and the ultimate objective of this project, namely, predicting the service life and remaining strength of HTLS conductors, could not be achieved without all the tasks being performed as part of one project. The research involves the following efforts:

- Thermomechanical evaluation and physical characterization of aluminum alloys used for the fabrication of HTLS conductors.

- Development of time-dependent failure criteria and experimentally-based inelastic constitutive models.

- Incorporation of models into computational tools for parametric studies, predictions of remaining life and analyses of structural tests.

- Confirmatory structural validation evaluation from NDE or FEM simulation.

- Investigation of microstructural evolution of aging conductors and their associated components.

Figure 4-6 provides the basic layout of the project's technical plan. Task 1 provides the experimental data necessary for the development of the inelastic constitutive models and the establishment of failure criteria. Task 2 establishs inelastic deformation models that address damage accumulation and development of a strength criterion. Task 3 incorporates the inelastic constitutive models into computational tools to determine the complex state of stress and its evolution in the vicinity of the conductor and splice. In Task 4, structural validation evaluation were performed. Task 5 will provide the SEM and transmission electron microscope (TEM) data to generate the microstructural evolution models of the aging conductor materials. This information will be implemented into Task2 and Task3 for the constitutive model development. 
Due to budget constraints, Task 5 was not pursued; instead limited efforts were carried out for some physical property investigations.

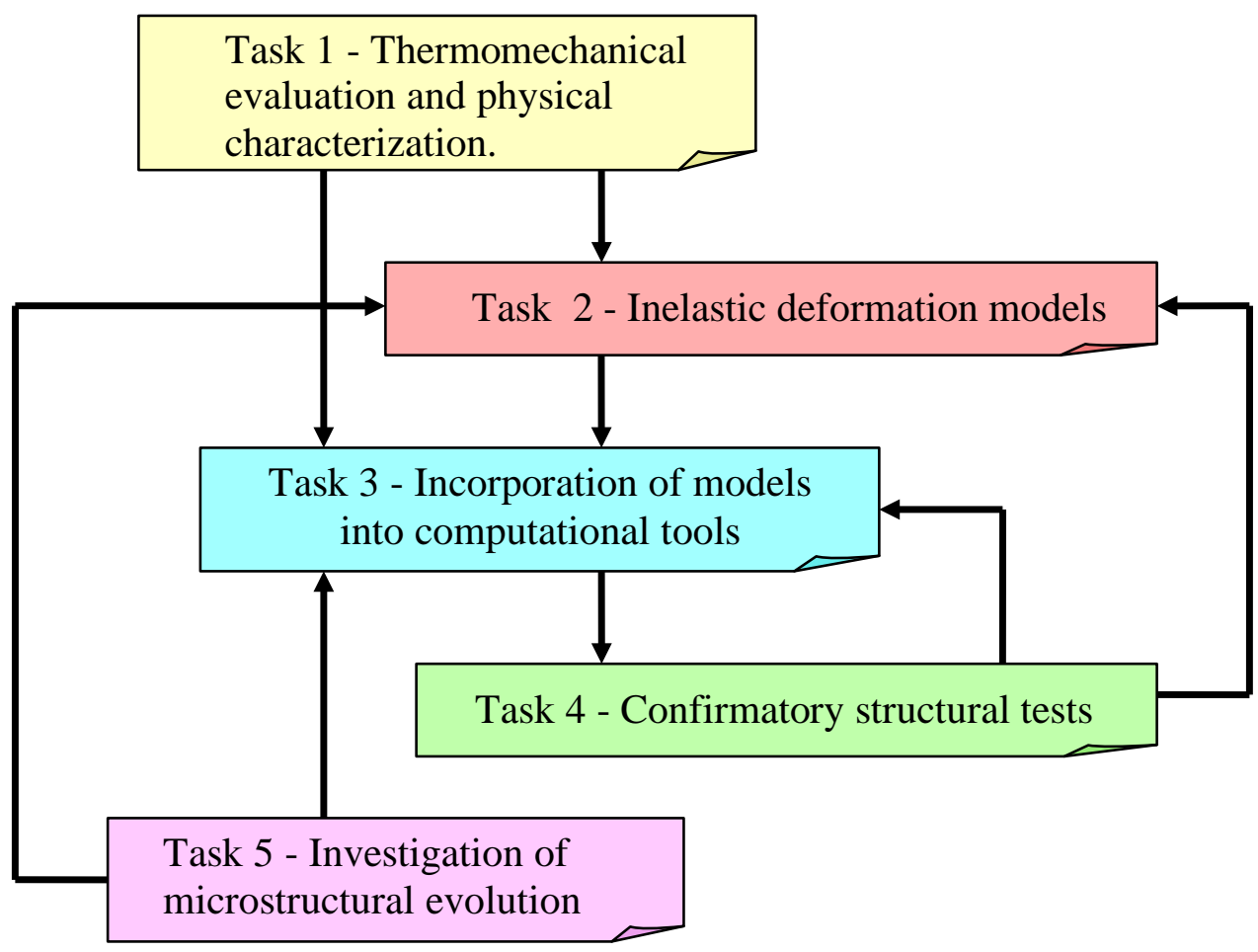

Figure 4-6 Layout of project technical plan.

\section{Task 1. Thermomechanical evaluation and physical characterization of aluminum alloys.}

This task provides experimental data for design and quantification of failure criteria and implementation of constitutive equations for inelastic design analyses. Mechanical tests were carried-out using servohydraulic and electromechanical testing machines equipped with digital controllers capable of programming complex loading histories, high-temperature furnaces and extensometers. Representative materials used for the reinforcing core and conductive phase of ACSR conductors and splice were evaluated at various temperatures while under conditions of creep-rupture. The strain-controlled low-cycle fatigue testing was carried out at various strain amplitudes, frequency and hold times. The specific parameters for these tests will be determined in coordination with the development of constitutive models. Operating experience will help establish the specific parameters.

\section{Task 2. Inelastic Deformation Models}

Failure models normally consist of two ingredients: (1) a damage accumulation model describing failure resulting from the accumulation of damage under varying thermal and mechanical loadings, and (2) a strength criterion. In the methodology presently covered by Section III, Subsection NH of ASME's Boiler and Pressure Vessel Code, the all-important area of creepfatigue failures is handled by separating damage into creep and fatigue components and provides 
an interaction diagram. Creep damage is characterized by time fractions, and fatigue by cycle fractions. The von Mises equivalent strain is used for the fatigue strength theory; a special equivalent stress quantity, which must be quantified for each material on the basis of multiaxial creep-rupture test results, is used for the creep-rupture strength theory. The linear-damage creepfatigue damage accumulation rule used in Subsection NH is the major shortcoming of existing high-temperature rules. Therefore, a key objective of this task is to identify an appropriate damage accumulation rule and associated strength theories for HTLS conductor materials.

Constitutive models, or equations, are the key ingredient of inelastic analysis methods. These equations describe the inelastic flow response of a material to complex time-varying loadings. The development of constitutive models for this project is based on results of a body of exploratory uniaxial tests in which specimens are subjected to a variety of relevant thermal and mechanical loading histories, to be carried out in Task 1. Therefore, efforts to develop constitutive and failure models need to be carried out in close coordination with Task 1 . The inelastic constitutive and failure models is validated and/or refined by coordinated modeling and testing efforts in Tasks 3 and 4.

\section{Task 3. Incorporation of models into computational tools}

While inelastic constitutive and failure models developed in Task 2 are capable of describing the evolution of stresses, strains and damage in conductor constituents, when they are subjected to geometrically uniform stresses/strains, those models by themselves were not capable of predicting the service life of HTLS conductors. This is a consequence of the fact that HTLS conductors are subjected to stresses and strains that are geometrically non-uniform in the vicinity of a splice and at other geometrical discontinuities. The magnitude and accumulation of strain and damage in this region of the conductor will evolve with time in a complex manner, as stresses are relaxed and/or redistributed due to creep and plastic deformation and strain and/or damage increase and accumulate after each thermal cycle or with time. The only viable approach to determine the magnitude and evolution of stresses and strains in that region of the conductor requires the use of the inelastic constitutive models coupled with finite-element modeling techniques. The commercially available finite element program ABAQUS was used for this purpose and is capable of incorporating the actual geometry of the conductor and a splice while modeling the deformation that is induced in the splice and conductor during assembly. The implementation of constitutive models in finite-element analysis tools provides the means for predicting the evolution of stresses and strains in HTLS conductors for any arbitrary loading history. For example, a loading history could include: the inelastic deformation induced during installation of the splice, the tensioning load introduced during erection of the conductor, temperature variations for a particular geographical location for which historical climatic data are available, and regular or irregular transients associated with increased power transmission during peak periods. The concentration of stresses, strains and damage in the vicinity of the spliceconductor interface region and their evolution will be investigated for a series of extreme scenarios to determine if simplified methods can be implemented to predict the service life of HTLS conductors.

While other secondary stresses, such as those introduced by aeolian vibrations, will not be considered explicitly in the proposed analysis, their effect on damage and deformation could be accounted for by an extension of the analysis. It has been documented that damage induced by aeolian vibrations tends to concentrate at the contact points between the conductor and vibration 
dampers, suspension saddles, insulators and aircraft warning markers. Therefore, knowledge of the state of stress of the conductor in the contact area with fittings, combined with a random spectral loading to simulate the time-dependence of aeolian vibrations and the constitutive description of the behavior of the conductor (to be developed in the proposed work), could be used to predict the effect of aeolian vibrations on the service life of conductors.

\section{Task 4. Confirmatory Structural Validation Evaluation}

Confirmatory, high-temperature, time-dependent evaluation was needed to (1) provide a better understanding of the structural behavior and failure modes, and (2) validate inelastic analysis methods. The structural component validation evaluation performed under this task incorporated real representations of the ACSR conductor structure system, including SSC system. The structural components were examined experimentally and analytically to provide deformation histories. Such deformation information was used to validate the finite-element analysis results obtained using the inelastic design models developed under Task 3.

\section{Task 5. Investigation of microstructural evolution of aging conductor materials}

This task will generate SEM and TEM microstructural data from the aging archived or simulated aging conductor materials and its associated components. The investigation topics will cover the grain and its associated boundary characteristics, the dislocation generation, motion, and annihilation, aluminum anodizing, and the electromigration microstructural damage mechanism. Such microstructure data will provide a base and guidelines for developing microstructure evolution models for the aging of archived conductors. Generated microstructural evolution models will be incorporated into Task 2 and Task 3 for developing constitutive equations for inelastic design analyses.

\subsubsection{Research Focus}

One of the primary questions regarding the use of HTLS conductors concerns their anticipated service life. That is, how long will they continue to perform satisfactorily? Upon reflection, the research team decided that this question was difficult to answer, even for conventional conductor, let alone HTLS. Therefore, the team decided to study the service life of conventional ACSR before trying to determine this for HTLS. When considering the service life of ACSR, it became apparent that the connectors and terminations of ACSR, especially full-tension single stage splice connectors and terminations, were the least robust component in the conventional conductor system. Therefore, an attempt has been made to determine the service life of single stage compression splices for ACSR.

\subsection{References}

1. Assessment of Conductor and Conductor Accessories Premature Aging from High Operating Temperature Cycling - State-of-the-Art Summary and Application Guideline, EPRI, Palo Alto, CA: 2004, 1008731. 
2. American National Standard for Electric Connectors: Connectors for Use Between Aluminum-to-Aluminum or Aluminum-to-Copper Bare Overhead Conductors, National Electrical Manufacturers Association, Rosslyn, VA: 1998. ANSI C119.4.

3. Aluminum Suspension Clamps, R Farley and D Paddon, Electrical Review, vol. 168, no. 14, pp. 652-653, 1961.

4. Effect of Elevated Temperature on the Performance of Conductor Accessories, W Howitt and T Simpkins, IEEE Paper C72 188-6, 1972.

5. Summary Report on the Effects of High Operating Temperatures on Conductors and Hardware Behavior, G Clarke, Ontario Hydro Research Division, Report No. 77-177-H, 1977.

6. O. Nigol and J. S. Barrett, "Development of an accurate model on ACSR conductors for calculating sags at high temperatures,” Ontario Hydro, CEA no. 78-93, March 1980.

7. Performance of Transmission Line Components at Increasing Operating Temperatures, EPRI, Palo Alto CA, December 2003.1002094.

8. Application Dynamics of High Temperature Conductors in Suspension Clamps, Tamm, Carl, Hubbell Power Systems

9. Applicability of resistance and temperature measurements for the characterization of full tension compression splices, Comte, C. and Lacasse, R, ESMO Proceedings 2003.

10. Suspension Clamp and ACSR Electrical Conductor Contact Conditions, Cardou, Alain, Leblond, Andre and Cloutier, Louis, Journal of Energy Engineering, April 1993

11. High Temperature Mechanical Properties of Bare Overhead Conductors, EPRI, Palo Alto, CA: 2000, 1000276.

12. Effect of Elevated Temperature Operation on the Strength of Aluminum Conductors, Harvey, J R, T 72 189-4 IEEE.

13. Effects of High Temperature Operation on Transmission Full-Tension Joints and Conductors, G Di Troia, Presented at Meeting 23 of CIGRE Study Committee 22-WG12, Evreux, France, August 26, 2000.

14. 'Loss of Strength of Overhead Electrical Conductors Caused By Elevated Temperature Operation, CIGRE SC22 WG 12 Loss of Strength WG22-12, Electra No. 162 October 1995"

15. Hotline Inspection and Control of Joints, Ormin, J. and Bartsch, J., CIGRE SC22-203 Session 1998"

16. Conductor and Associated Hardware Impacts During High Temperature Operations, Shan, L and Douglas, D, EPRI TR109044, December 1997" 
17. Investigation on the Aging of Old ACSR Cables in Transmission Lines : Microstructural Evolution and Loss of Strength, Rizzo Assuncao, FC, DeCarvalho, AHP, Samic, RAM, Rosa, PF, , CIGRE 1992 Session, 22-302"

18. Effect of Elevated Temperature Operation on the Tensile Strength of Overhead Conductors, Morgan, Vincent, IEEE 95 WM 229-5.

19. Overhead Conductor Manual, Southwire Company

20. Application Dynamics of High Temperature Conductors in Full Tension Splices \& Deadends, Tamm, Carl R, Hubbell Power Systems, Transmission and Distribution Conference and Exposition, IEEE PES. 2003.

21. M. Runde, E. Hodne, B. Totdal, "Current-induced aging of contact spots" in proceedings of the 35th IEEE Holm-Conference on Electrical Contacts, 1989, pp. 213-220.

22. R. Bergmann, H. Böhme, H. Löbl, S. Großmann, "Model to asses the reliability of electrical joints" in proceedings of the 18th International Conference on Electrical Contacts, 1996, pp. 180-188.

23. M. Braunovic and N. Aleksandrov, "Intermetallic compounds at aluminum-to-copper and copper-to-tin electrical interfaces" in proceedings of the 38th IEEE Holm Conference on Electrical Contacts, 1992, pp. 25-34.

24. J. Aronstein, “AC and DC electromigration in aluminum contact junctions” in proceedings of the 18th International Conference on Electrical Contacts, 1996, pp. 311320.

25. M. Braunovic "Critical slip amplitude required for initalisation of fretting motion in a binding-head screw termination" in proceedings of the 20th International Conference on Electric Contact Phenomena, 2000, pp. 373-377.

26. S. Großmann, J. Kindersberger, H. Löbl, S. Schoft, “Creep ageing of bolted electrical busbar joints" in proceedings of the 19th International Conference on Electric Contact Phenomena, 1998, pp. 269-273.

27. J. Kindersberger, H. Löbl, S. Schoft, "Plastic deformation and loss of joint force by creep in high current joints" in proceedings of the $20^{\text {th }}$ International Conference on Electric Contact Phenomena, 2000, pp. 367-372. 



\section{THERMAL-MECHANICAL PROPERTIES EVALUATION FOR ACSR SSC SYSTEM COMPONENTS}

A schematic diagram of a half SSC and the associated materials are shown in Figure 5-1, where the SSC splice connector can be divided into two major sections, namely, a conductor section that contains the aluminum conductor (section $\mathrm{C}-\mathrm{C}$ ), and a core-grip section (section B-B). The conductor wires are made from 1350-H19 aluminum, the SSC sleeve is made of 3003-H183 aluminum, and the core-grip is made of 6061-T6 aluminum. The thermal-mechanical properties investigation of the SSC system components are described below. More test results from thermal-mechanical property investigations are provided in Appendix C.

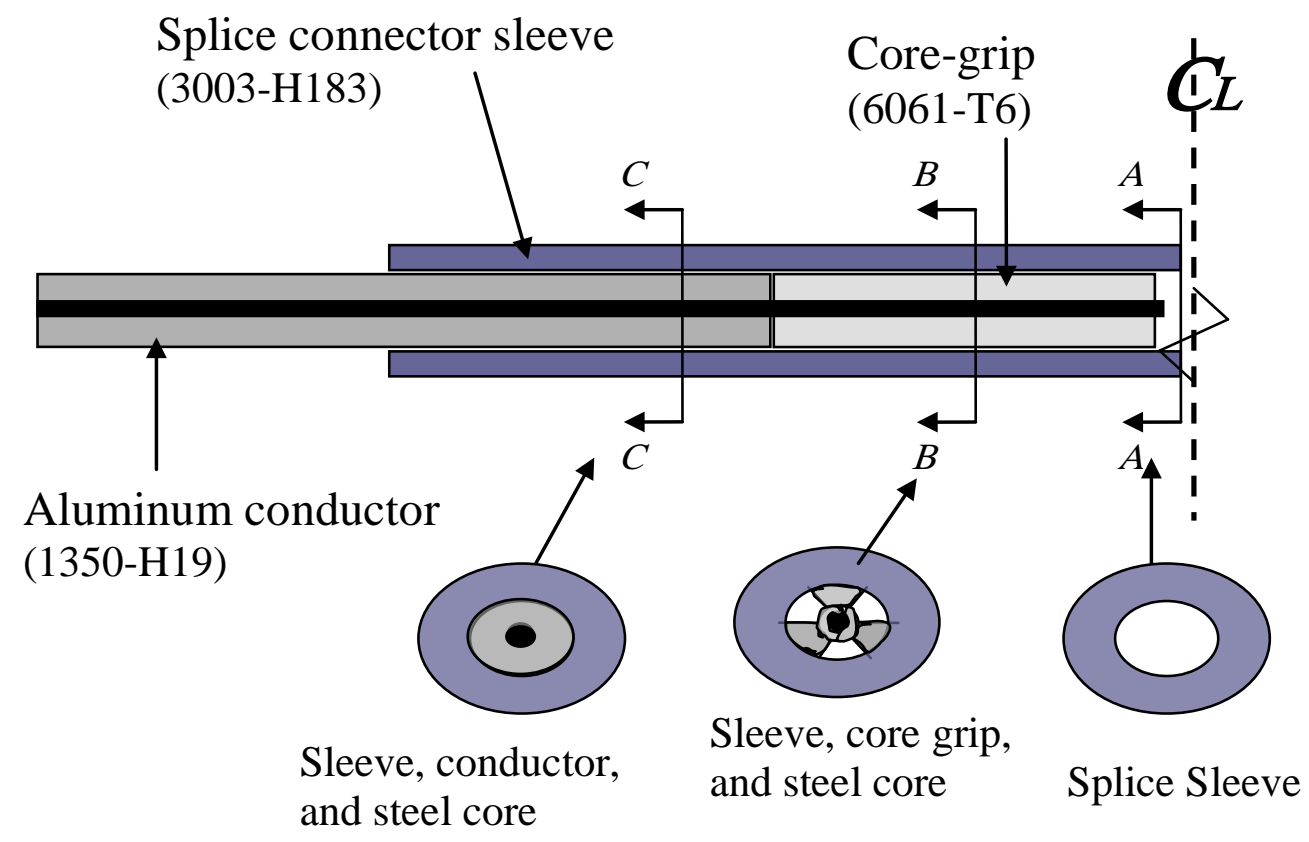

Figure 5-1 Detailed cross-section of a half ASCR SSC fitting.

\subsection{The Preparation of Test Set-Up for Tensile, Creep, Fatigue, and Thermo- Mechanical Fatigue Testing}

The test machines available for the research project are:

1. Servohydraulic biaxial (axial/torsion) testing machine MTS Mod. 809: Axial capacity = 100 KN (22Kip), Torsion Capacity = 1100 N-m (9,750 lb-in)

2. Servohydraulic uniaxial (axial) testing machine MTS Mod. 810 Material Test System with hydraulic wedge grips: Force capacity $=25 \mathrm{KN}$ (5.5Kip)

3. Three units (two by ATS Inc. and an ORNL-build) of standard, two-poster, pneumatic powered, universal testers are also available for creep properties testing l. The maximum 
capacity of the machines is $5,000 \mathrm{lbf}$

4. 5 cantilever creep test machines with furnaces: Ratio between the dead weight and the applied load $=1: 12$, using LVDTs to record displacement

The set-ups with the associated instrumental control for items 1-4 are were calibrated before conducting the testing. Testing can be performed at elevated temperatures above ambient with a two-zone controlled furnace. The load train assembly can also be insulated in a cold box for cyclic temperature testing below ambient temperatures with chilled nitrogen gas. When using the ATS machine, creep strain is measured with an optical strain extensometer, which has been demonstrated to provide the good long-term stability required for these types of applications.

\subsubsection{Test Set-up for Tensile and Fatigue Testing at High Temperatures}

The Item 2 test set-up uses a high temperature environmental chamber and temperature control unit (red box) as illustrated in Figure 5-2. Due to the large elongation of the 1350-H19 aluminum at high temperature, an integrated set-up of combining Laser Mike and the conventional extensometer was designed and used for measuring the deformation strain; Figure 5-3 shows the extensometer end that contacts the specimen in the environmental chamber (furnace), and Figure 5-4 shows the Laser Mike set-up with the calibration band attached at the right end of extensometer. A closer-look of tensile testing in a high temperature environment is shown in Figure 5-5. The details of tester alignment are shown in Figure 5-6, where the initial alignment was done with a dial gage, shown in Figure 5-6 (a), and fine tuning was done with a dummy specimen equipped with several strain gages in the gage length to monitor the stress induced by misalignment of the angle and eccentric of the loading train, as shown in Figure 5-6 (b). The details of set-up for calibrating the extensometer and the attached Laser Mike calibration band are shown in Figure 5-7.

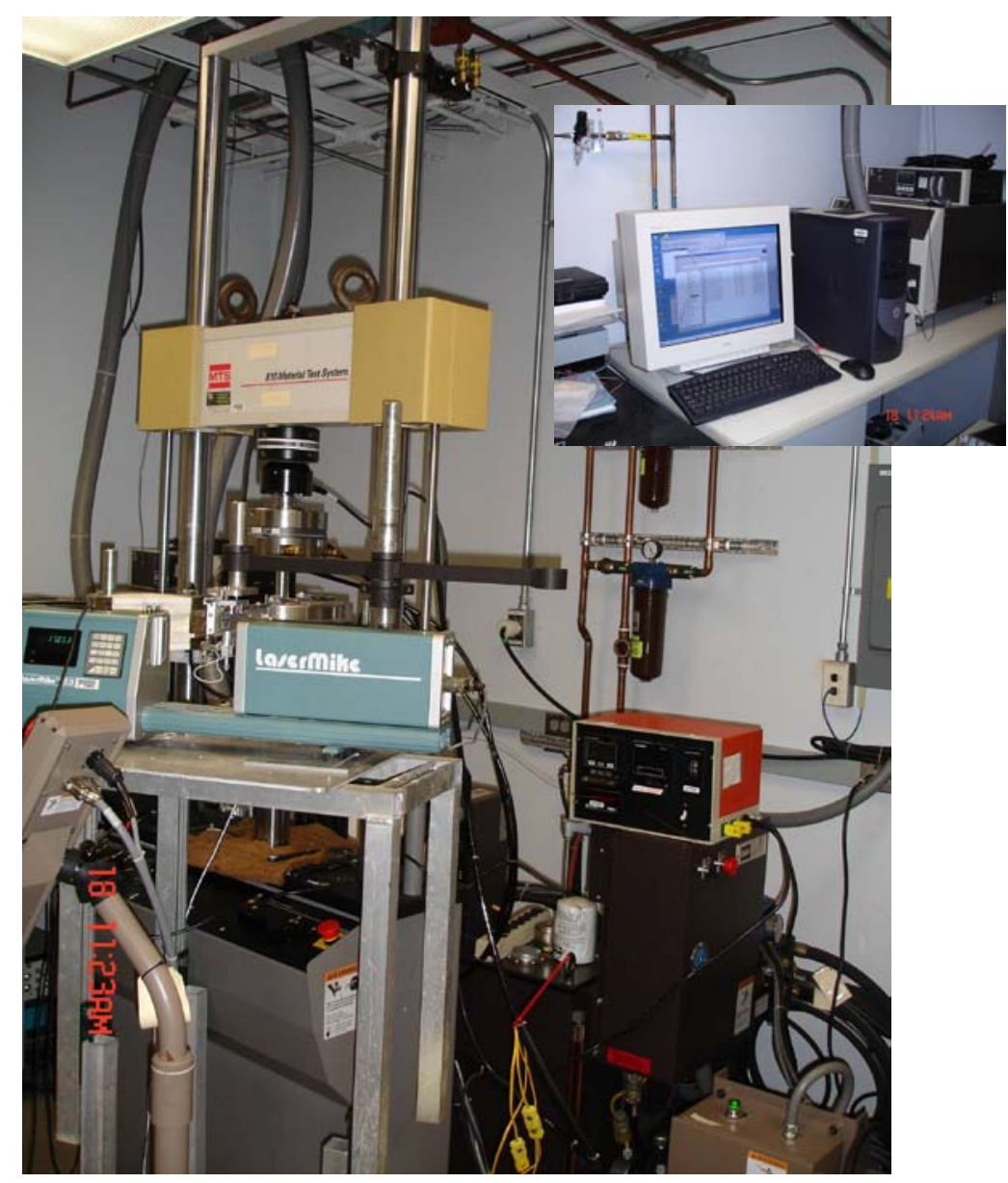

Figure 5-2 Tensile and fatigue test set-up: environmental chamber, temperature and load controls and data acquisition system. 


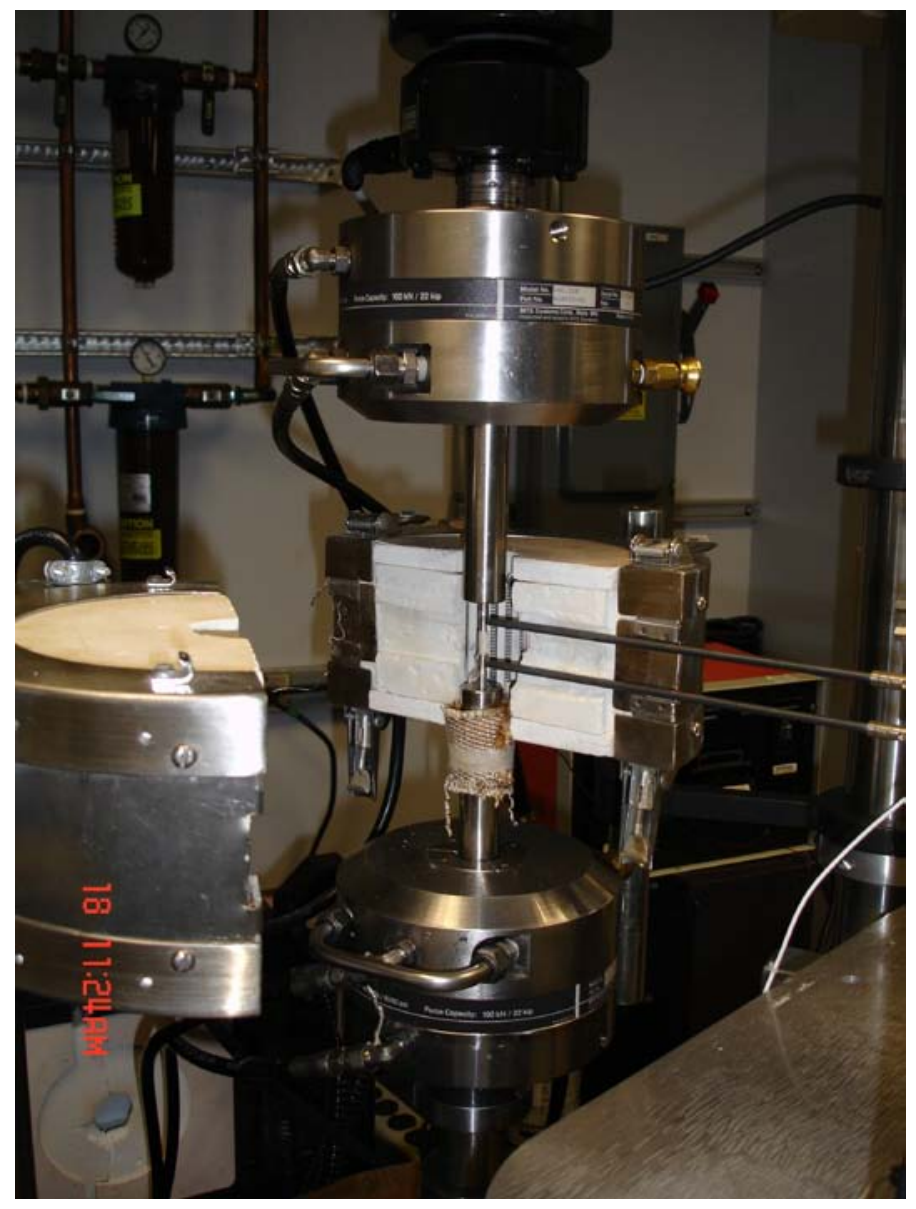

Figure 5-3 Close-up of environmental chamber and the extensometer, which is part of integrated test controls system for measurement deformation.

Figure 5-4 Close-up of the Laser Mike and the calibration band attached at the end of extensometer for strain measurement.

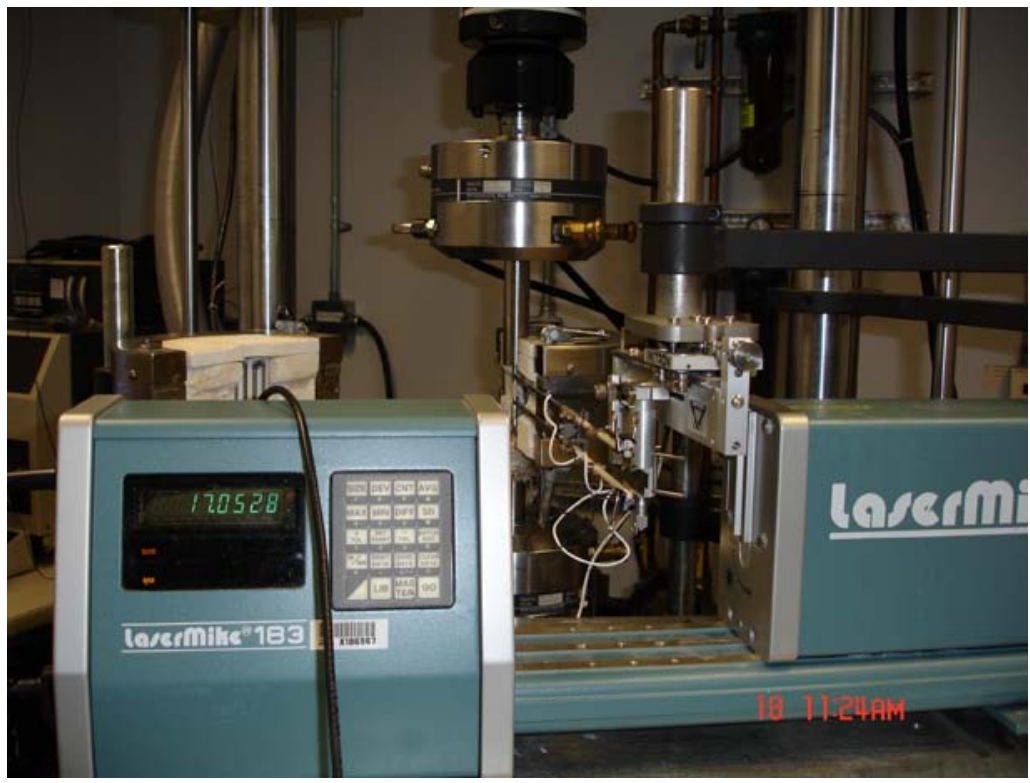




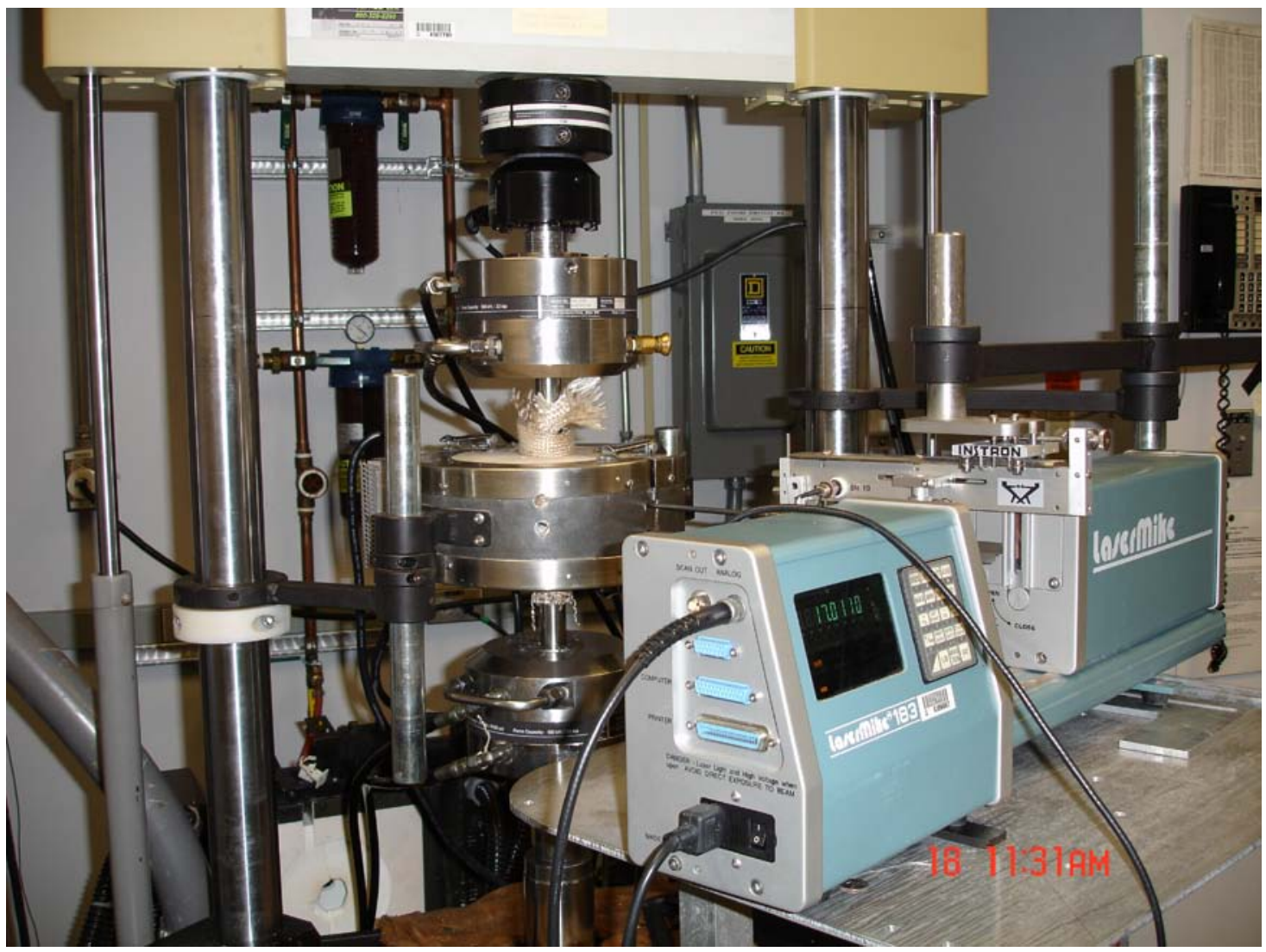

Figure 5-5 High temperature tensile test with the associated deformation control set-up. 


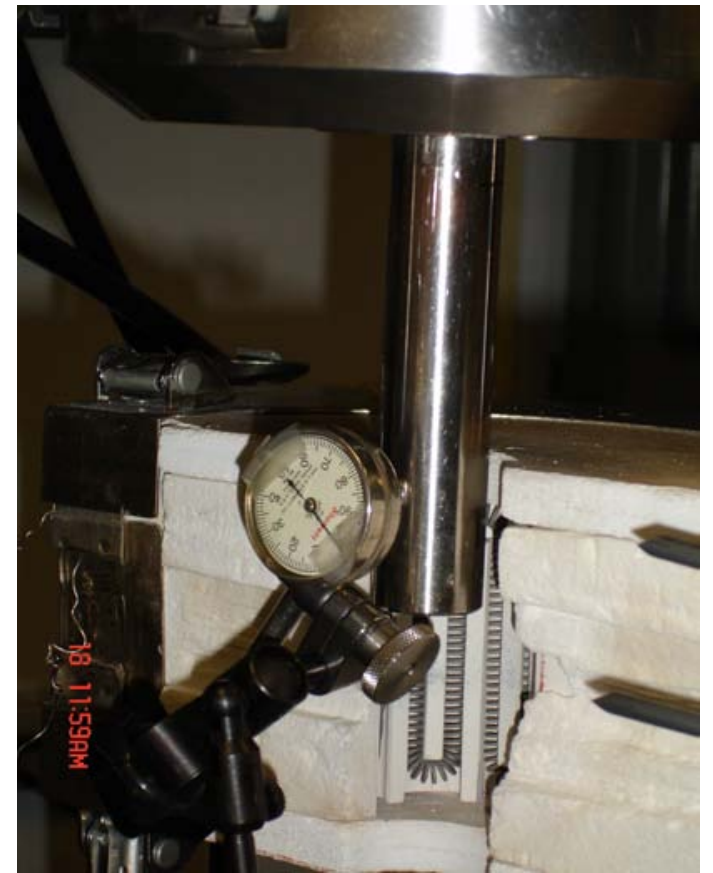

(a)

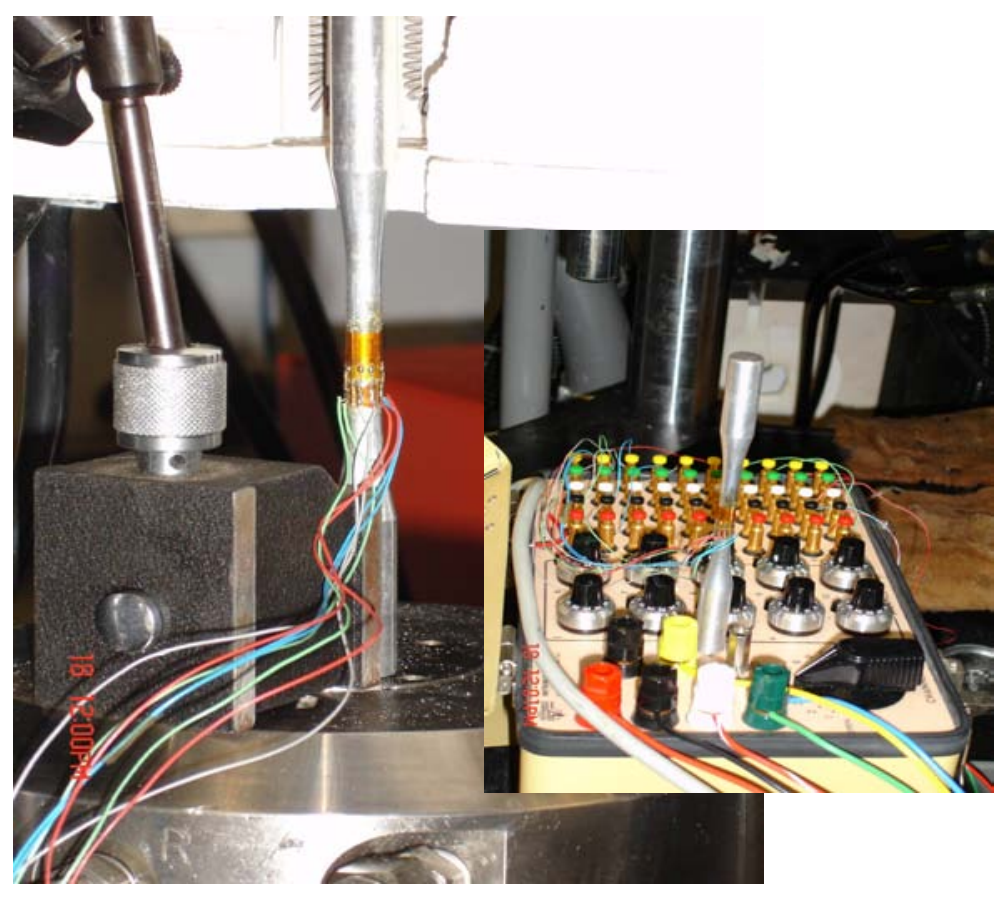

(b)

Figure 5-6 (a) Close-up of the dial gage used in the initial alignment of the tester loading train, and (b) set-up for fine tuning the tester alignment, where a dummy specimen equipped with strain gages is used to detect the stresses induced by the misalignment of the loading train. The associated control box is also shown in (b).

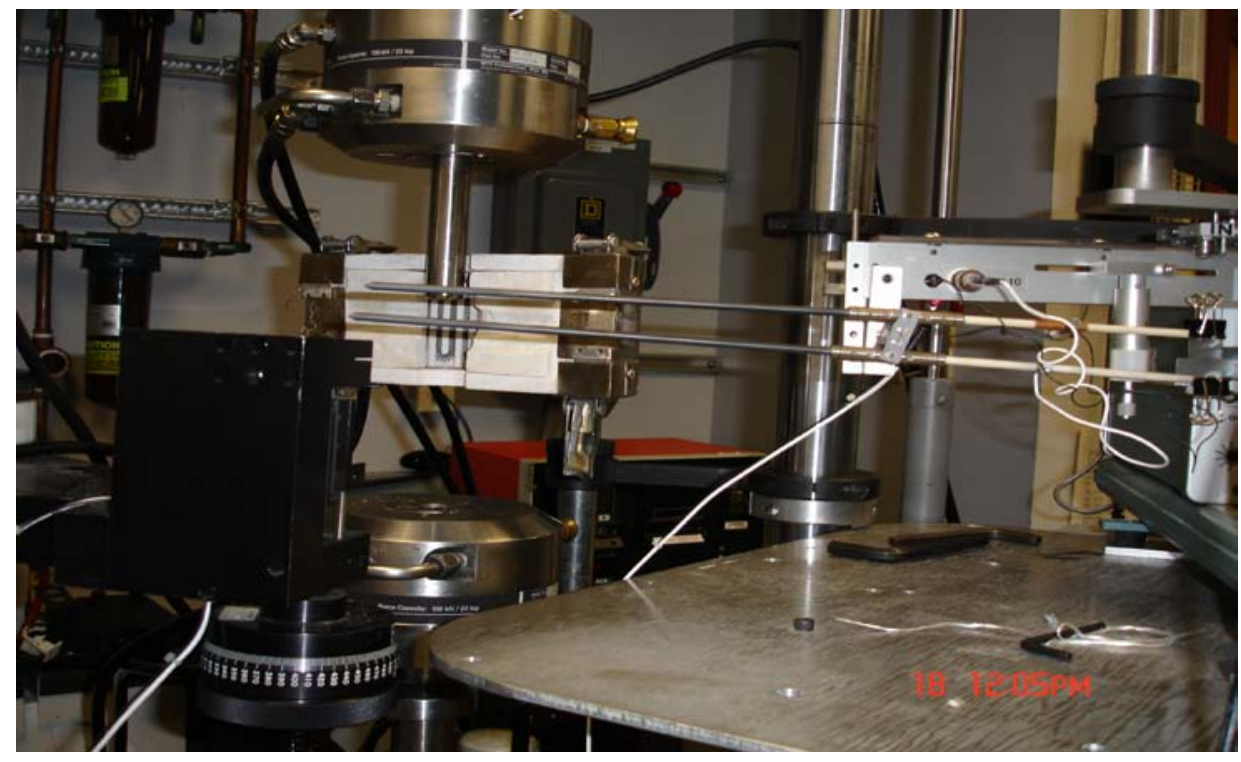

Figure 5-7 Set-up for calibrating the extensometer with the attached calibration band. The calibration block is shown in the bottom left and the Laser Mike calibration band is shown to the right at the end of extensometer. 


\subsubsection{Test Set-up of ATS Creep Machines and Cantilever Creep Tester}

The ATS creep machine set-up with the environmental chambers and instrument control systems are shown in Figure 5-8. More details of the system control and the interior of the environmental chamber are shown in Figure 5-9.

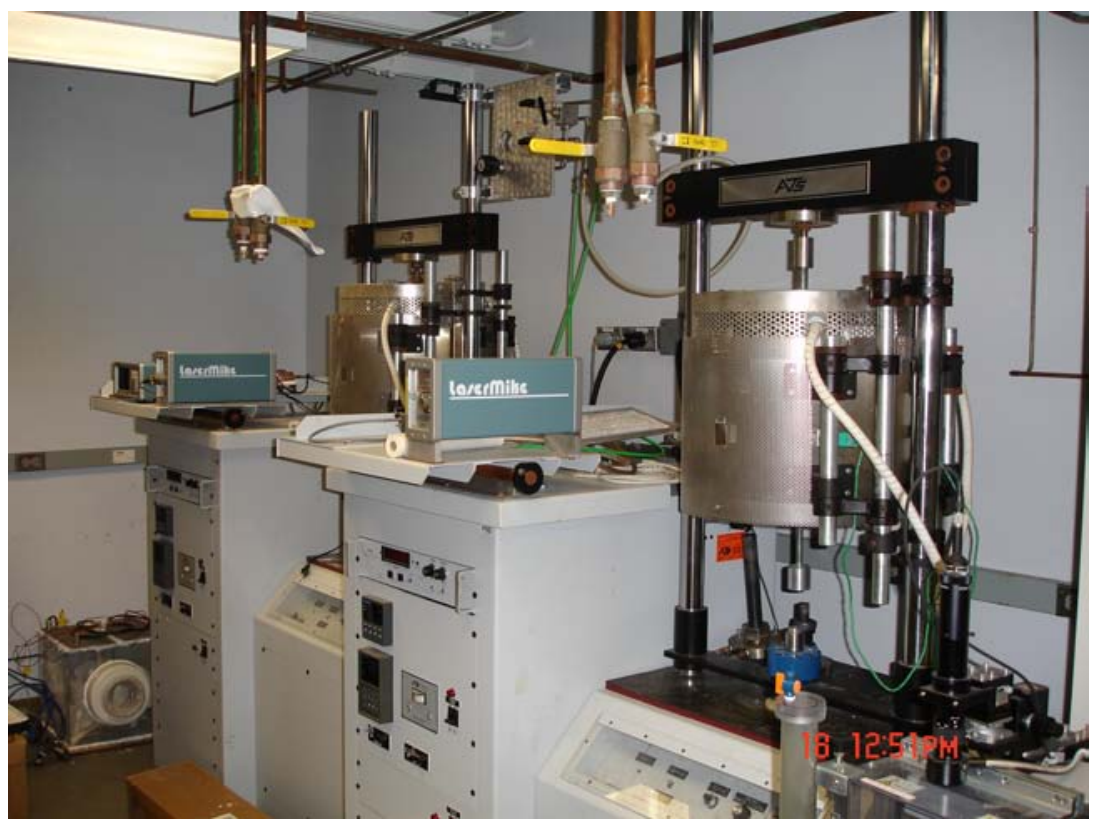

Figure 5-8 ATS creep test machine's set-up with the environmental chamber and the control systems for heating, loading and deformation measurement.

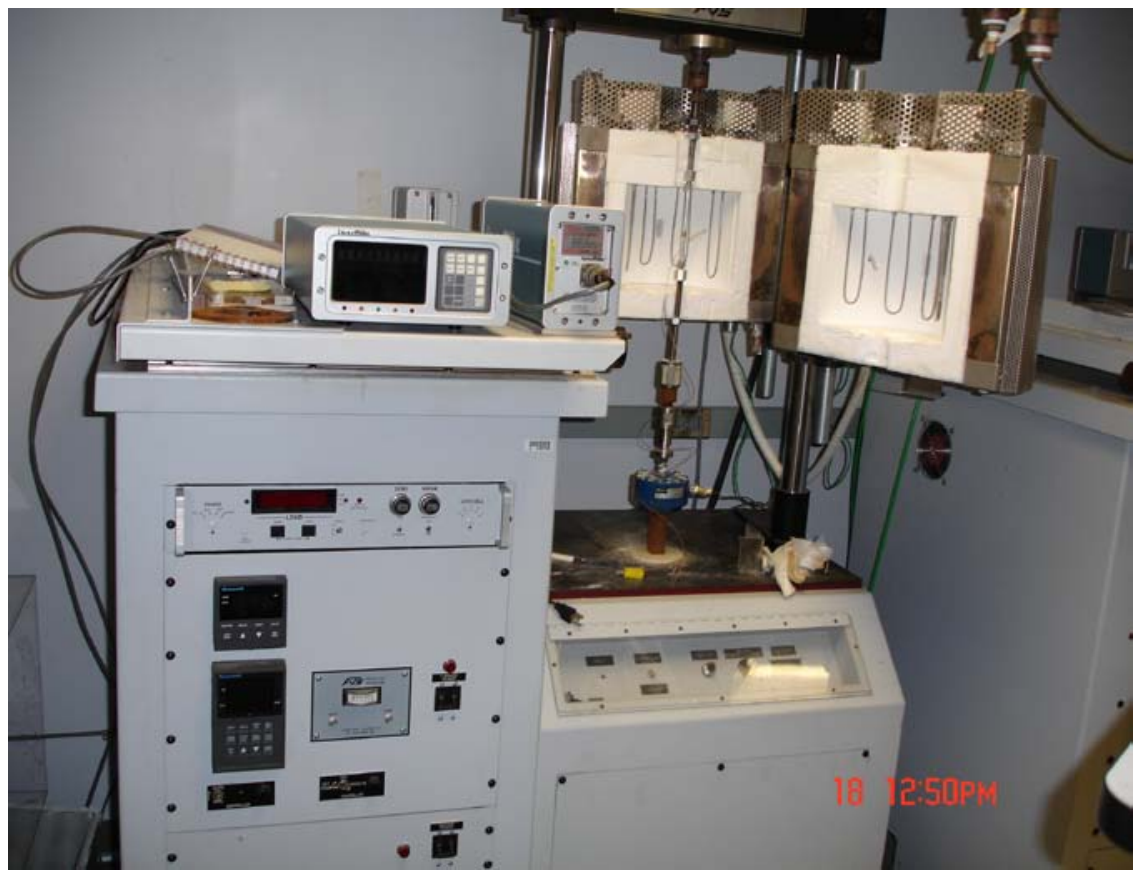

Figure 5-9 A close-up of one ATS creep test machine, with a visual of the interior of the environmental chamber. The left tall cabinet is the furnace and temperature control system and the right cabinet is the load control system. 
The cantilever creep tester's set up is shown in Figure 5-10.

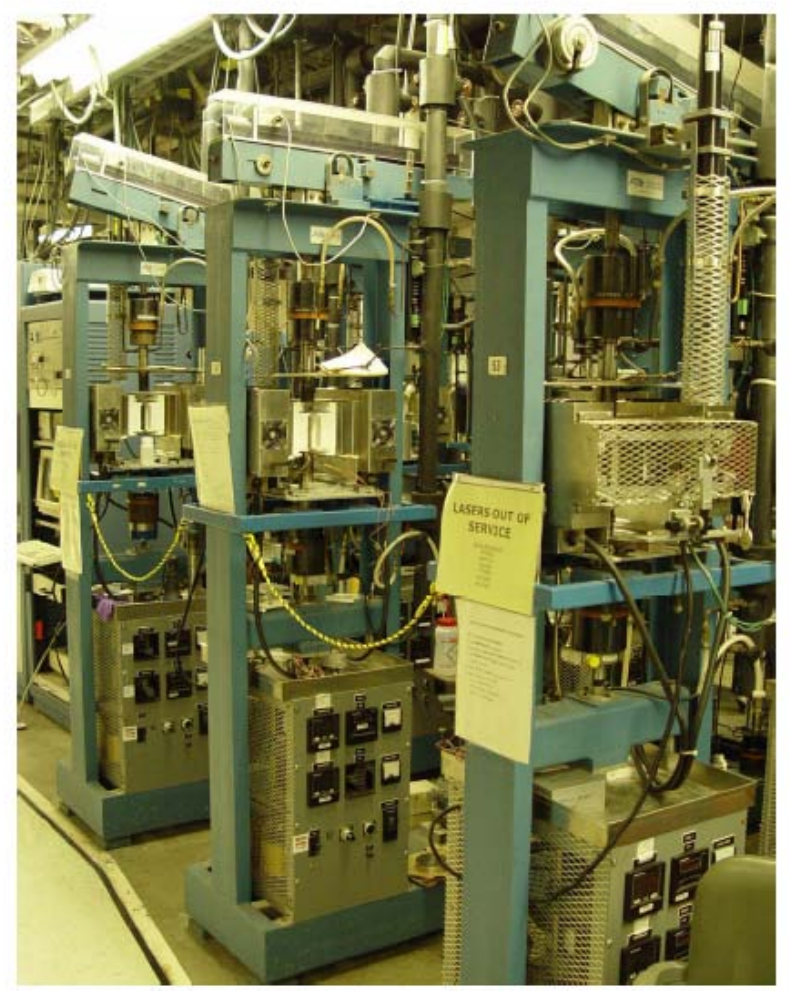

Figure 5-10 Cantilever creep tester set-up

\subsection{PILOT TENSILE TESTS ON 1350-H19 CONDUCTOR MATERIAL}

\subsubsection{Manufacturing 1350-H19 Aluminum Material}

Several pilot tests were conducted on the first order of the 1350-H19 material. However, the initial test results indicated that the received wire only has $14 \mathrm{ksi}$ tensile strength and is not in the category of ultimate tensile strength for 1350-H19 aluminum, instead it more resembles that of the fully annealed 1350-O aluminum. The received first order is shown in Figure 5-11, even though we had specified that the ordered wire should be wrapped in a relatively large barrel, at least 24-36 inch in diameter, to avoid further bending of the wire. But, the barrel of the received wire had only an 8 inch in diameter; clearly this is also not in an acceptable form for machining the test samples, in addition to the lack of the strength. Thus, the first order of the raw aluminum material was discarded.

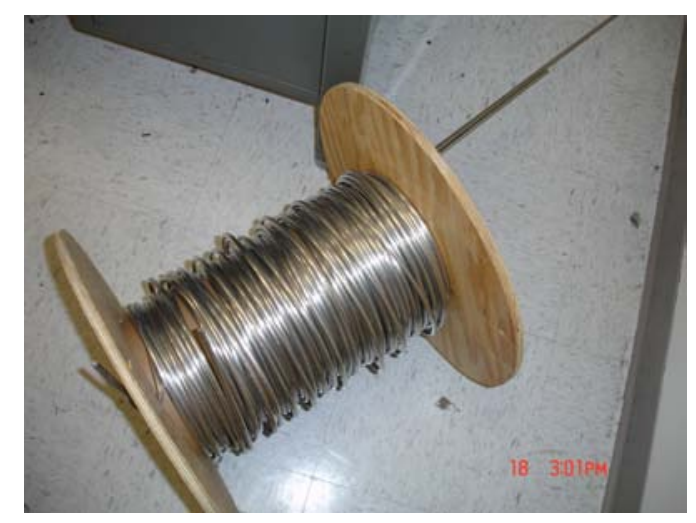

Figure 5-11 First order of 1350 aluminum shipped with an 8 inch diameter barrel. 
Exhaustive efforts to seek straight and large diameter of the 1350-H19 wire from commercially available vendors were in vain. We decided to have this material manufactured using the same cold drawing process used to produce the 1350H19 wire. To ensure the raw material is in a straight form for machining the test samples, this special order was manufactured and shipped in a straight rod form of 10 -foot in length. The received second order of the $1350-\mathrm{H} 19$ rod with 0.28 inch diameter is shown in Figure 5-12

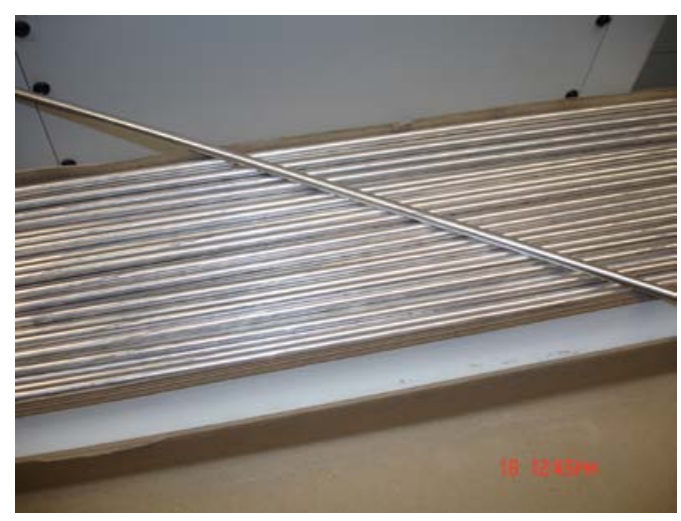

Figure 5-12 Second order of 1350-H19 rod with \#1 AWG size, at 0.28 inch diameter.

\subsubsection{Pilot Testing Set-up and the Associated Specimen Grip Design of the Received Aluminum 1350 Material}

Both the pilot tensile testing for the first order and the second order of the 1350 Aluminum material were carried out at ATS machines, which is a pneumatic powered, universal tester. The ATS machine test set-up and the associated loading train is shown in Figure 5-13. The pipe fitting used as the end grip of the test specimen in a loading train is shown in Figure 5-14.

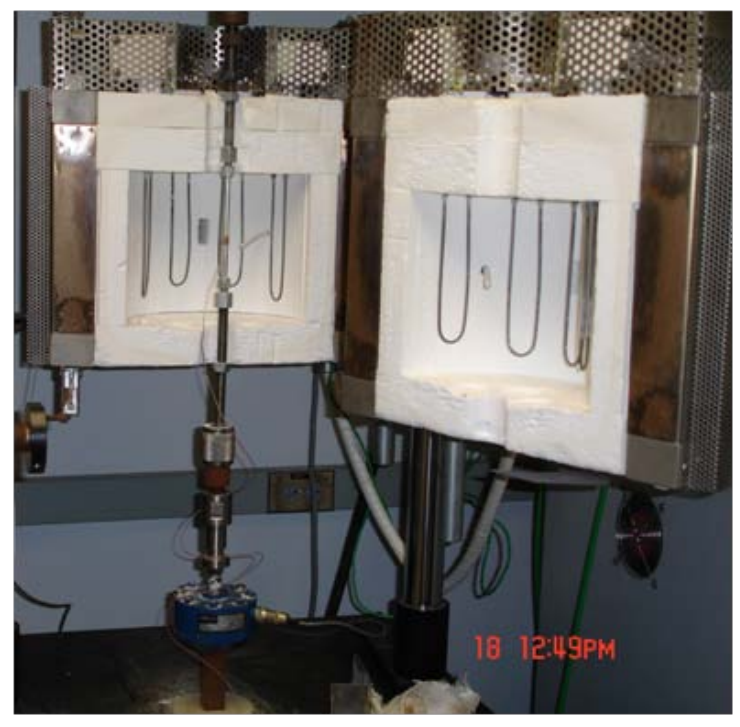

Figure 5-13 Pilot tensile testing set-up in an ATS creep machine with the associated loading train.

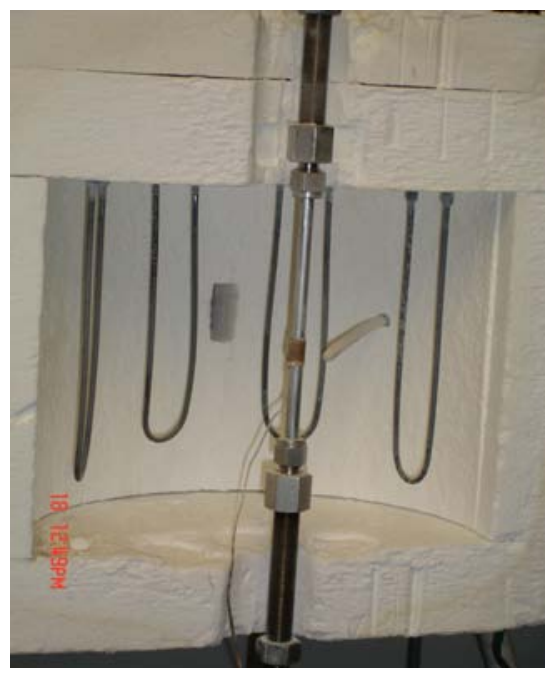

Figure 5-14 The end-grip design for pilot tensile testing.

The end-grip design and the fracture sample from the pilot testing for the first batch of the ordered 1350 aluminum are shown in Figure 5-15. It is noted here that a relatively large extension was observed during the tensile test and necking failure almost to a point; and the 
initially bent specimen was straightened after the tensile test. The ultimate tensile strength is estimated about 14 Ksi.

The pipe fitting end-grip design was also used for pilot testing of the 1350-H19 aluminum rod. The vendor's quality control data indicated that this received material has about $21 \mathrm{ksi}$ tensile strength. The rod was first machined down to 0.25 -inch diameter before it was fitted into a $1 / 4$ inch pipe fitting. During the tensile testing, none of the specimens were failed by this design. The end grip would start to slip at about $800 \mathrm{lb}$ tensile loading. It was determine the maximum load capacity by the pipe fitting of $1 / 4$ inch is about $800 \mathrm{lb}$, and it is not suitable for a harder specimen as that of 1350-H19 rod. Figure 5-16 shows typical sample with failed grip end. The initial biting mechanism of the pipe fitting ring provides the ultimate grip strength to the test sample.

However, the material strength is obviously higher than the biting grip strength and results in the slippage at the grip-end when the threshold biting grip strength loading is reached during tensile testing. The slippage phenomenon of the end grip forms a reduced section at the specimen gripend as indicated in the right hand side of the test specimen, shown in Figure 5-16.

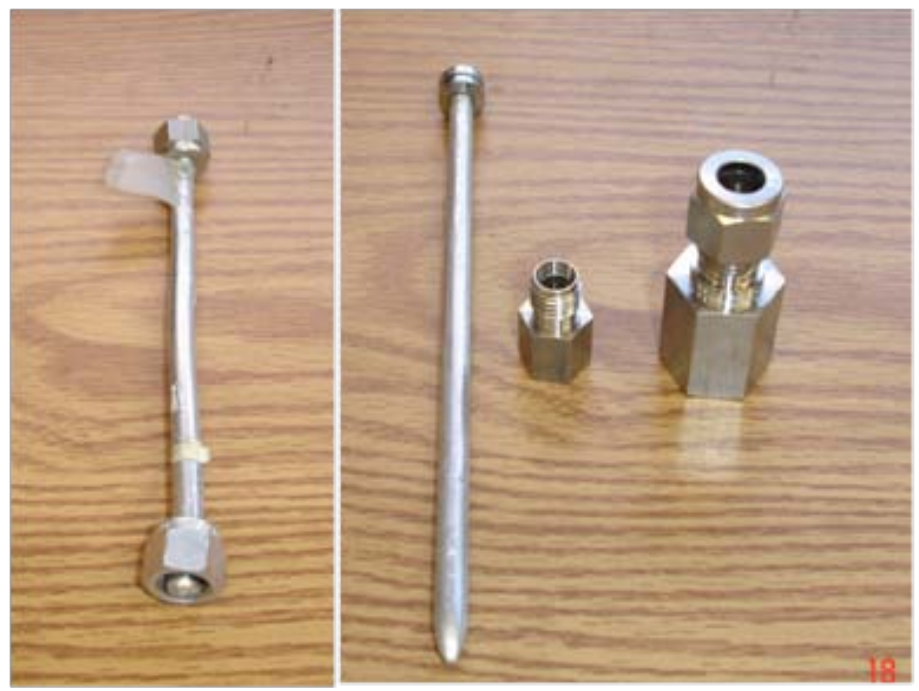

Figure 5-15 The fractured sample of the pilot tensile testing for the first order of $1350-0$.
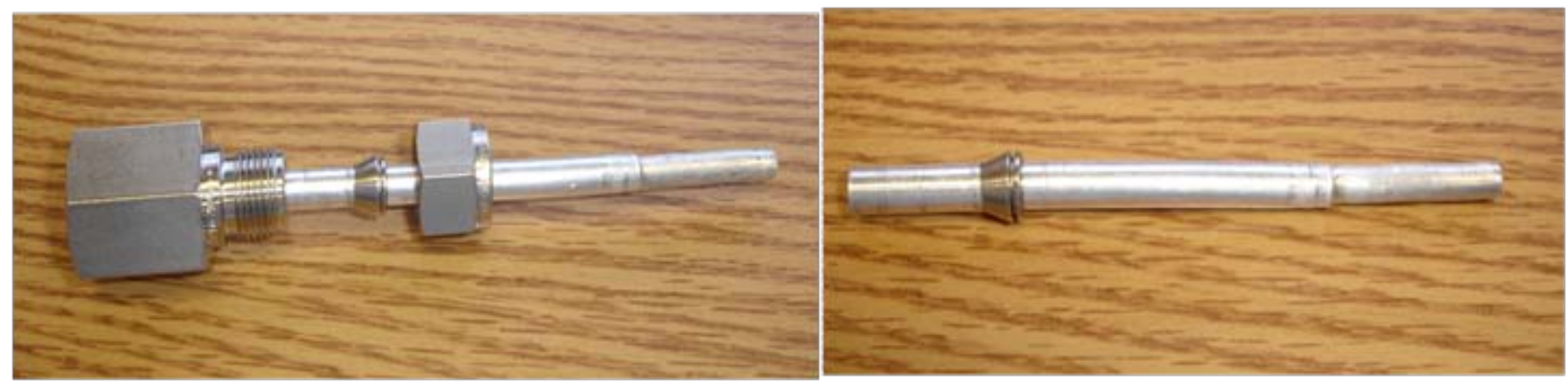

Figure 5-16 (Left) The typical slippage failure of 1350-H19 rods at grip-end during pilot tensile testing. The reduced section at the grip-end is due to a pipe fitting ring bitten into the testing sample. (Right) Details of a tensile sample failed at grip-end, and the intact side with the pipe fitting ring.

Based on this failure experience, a new specimen design was initiated and described below. 


\subsubsection{New Specimen Designs for Tensile and Fatigue Testing of 1350-H19 Rod}

The thread-end design was used for tensile and fatigue specimen designs, where the tensile specimen has 1 inch gage length and 0.5 inch for fatigue samples. The details of the first batch specimen for tensile and fatigue testing are shown in Figure 5-17 and Figure 5-18.

\section{TENBLH FATIGESFEOMEN}

\section{SCALE $=21$}
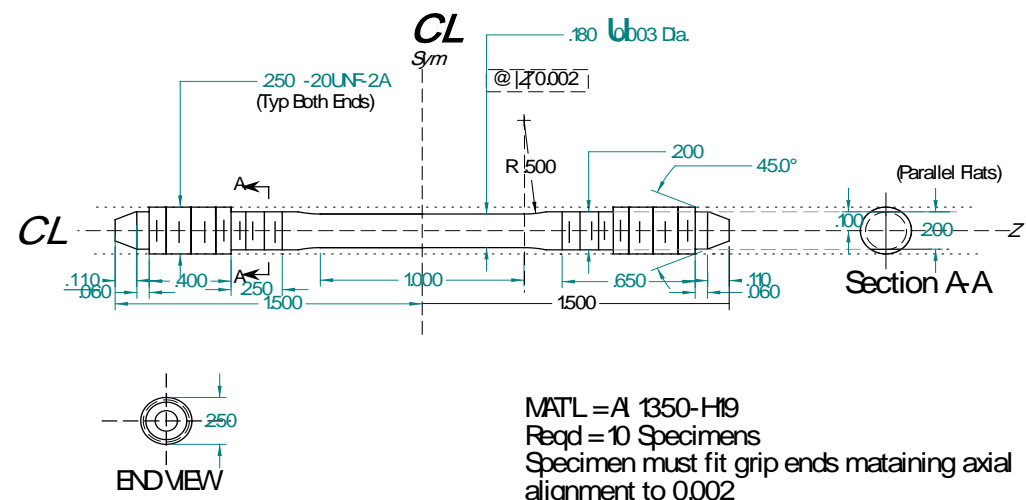

$M A T L=A 1350-H 19$

Reqd $=10$ Specimens

Specimen must fit grip ends mataining axial

alignment to 0.002

All dimensions are in inch

John Wang 10-27-2005

Call574-2274 for questions

Figure 5-17 Tensile Specimen Design with 1 inch gage length and 0.18 inch gage diameter.

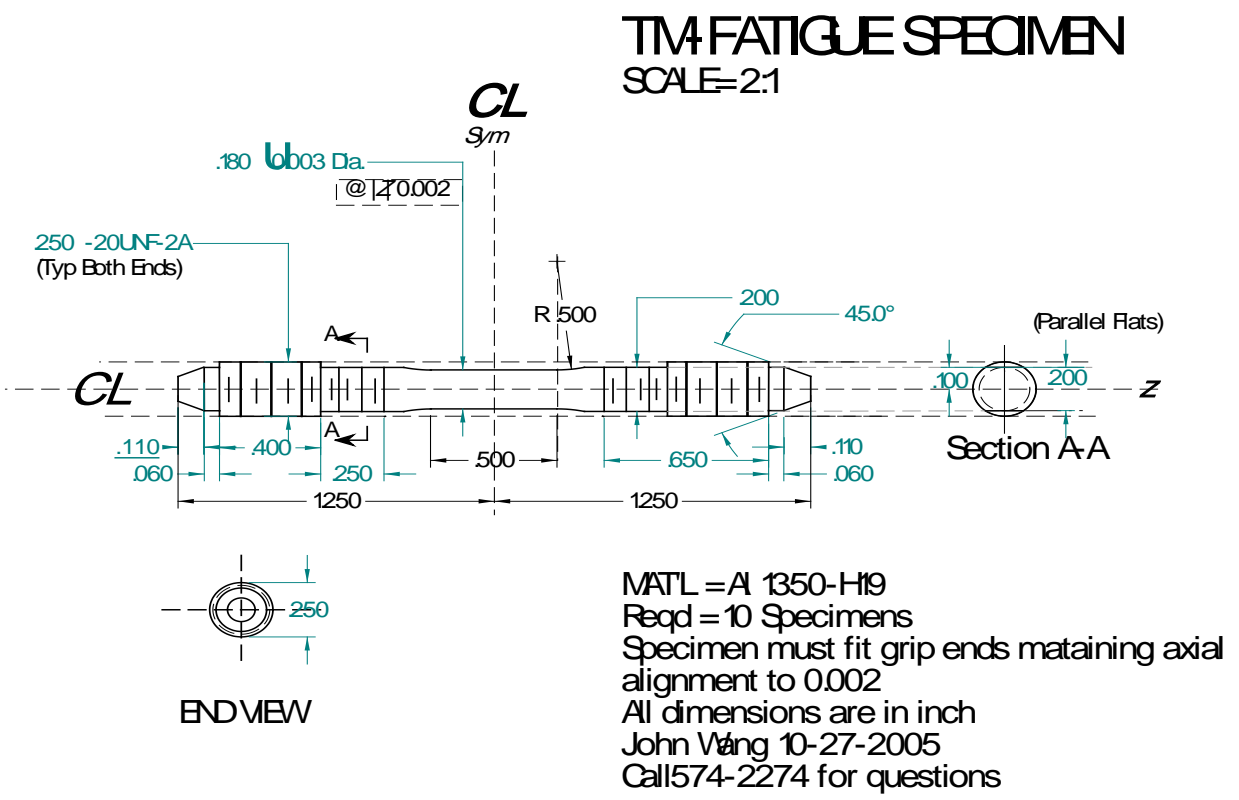

Figure 5-18 Fatigue Specimen Design with 0.5 inch gage length and 0.18 inch gage diameter.

The end grip design for the tensile and fatigue specimens is shown in Figure 5-19. 


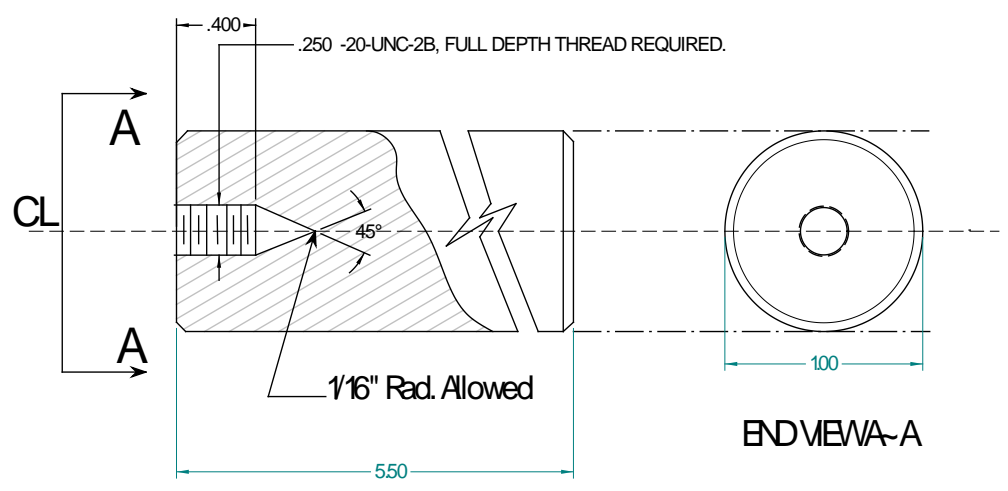

PUSHPUL ROD SCAL21
MATL SST

FEQDTwo per assembly

John Vłang 10-27-2005

Questions call 574-2274

Figure 5-19 End grip design for tensile and fatigue testing of the 1350-H19 rod.

The short gage-length design for the fatigue sample was mainly due to concerns regarding the potential of the test specimen to buckle during the compression cycle. The initial tests of the tensile sample with one inch gage length indicated a high probability of buckling of the test sample under compression. The tensile tests were conducted on the first batch of the designed tensile specimen. Figure 5-20 illustrates the typical specimen fracture or specimen necking under tension loading. The top left of Figure 5-20 shows the fracture sample with the associated end grip, and the thermal couple attached to the fractured specimen. The bottom picture shows the typical cone shape failure mode of the fractured tensile specimens.

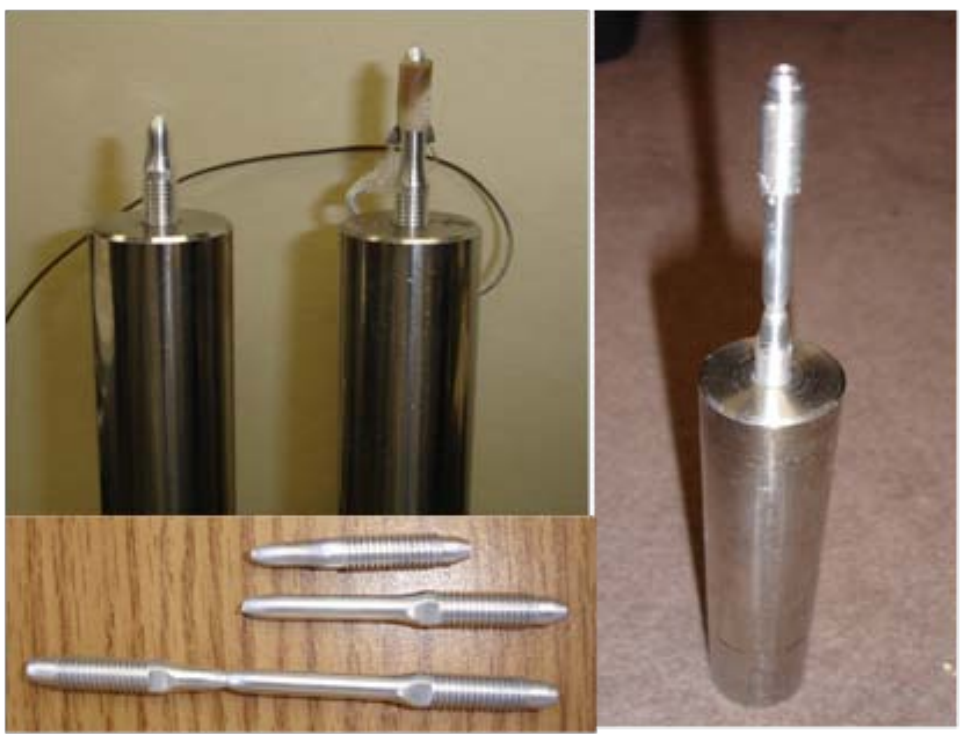

Figure 5-20 Fractured tensile specimens and end-grip. 


\subsubsection{Tensile Test Results for the $1350-H 19$ Rod Material at $20^{\circ} \mathrm{C}, 120^{\circ} \mathrm{C}$, and $200^{\circ} \mathrm{C}$}

Tensile tests were conducted at $20^{\circ} \mathrm{C}, 120^{\circ} \mathrm{C}$ and $200^{\circ} \mathrm{C}$ at the strain rate of $0.4 \% / \mathrm{min}$. At this loading rate, slight stress relaxation or the material creep was observed during the tensile testing at higher temperature. The material properties obtained from the tensile tests are summarized in Table 5-1 Summary of the material properties for the 1350-H19 aluminum rod.

Table 5-1 Summary of the material properties for the $1350-\mathrm{H} 19$ aluminum rod

\begin{tabular}{|l|l|l|l|}
\hline Temperature, ${ }^{\circ} \mathrm{C}$ & Young's Modulus, ksi & Yield stress, ksi & Tensile Strength, ksi \\
\hline $20^{\circ} \mathrm{C}$ & 10,000 & 20.5 & 22.2 \\
\hline $120^{\circ} \mathrm{C}$ & 8,000 & 18.5 & 20.5 \\
\hline $200^{\circ} \mathrm{C}$ & 7,000 & 16.7 & 18.0 \\
\hline
\end{tabular}

Table 5-1 indicates that there are about 30\% reduction in Young's modulus and about 19\% reduction in both yield stress and ultimate tensile strength from $20^{\circ} \mathrm{C}$ to $200^{\circ} \mathrm{C}$. The summary of the stress-strain cures of the typical tensile tests for 1350-H19 aluminum rod are shown in Figure 5-21.

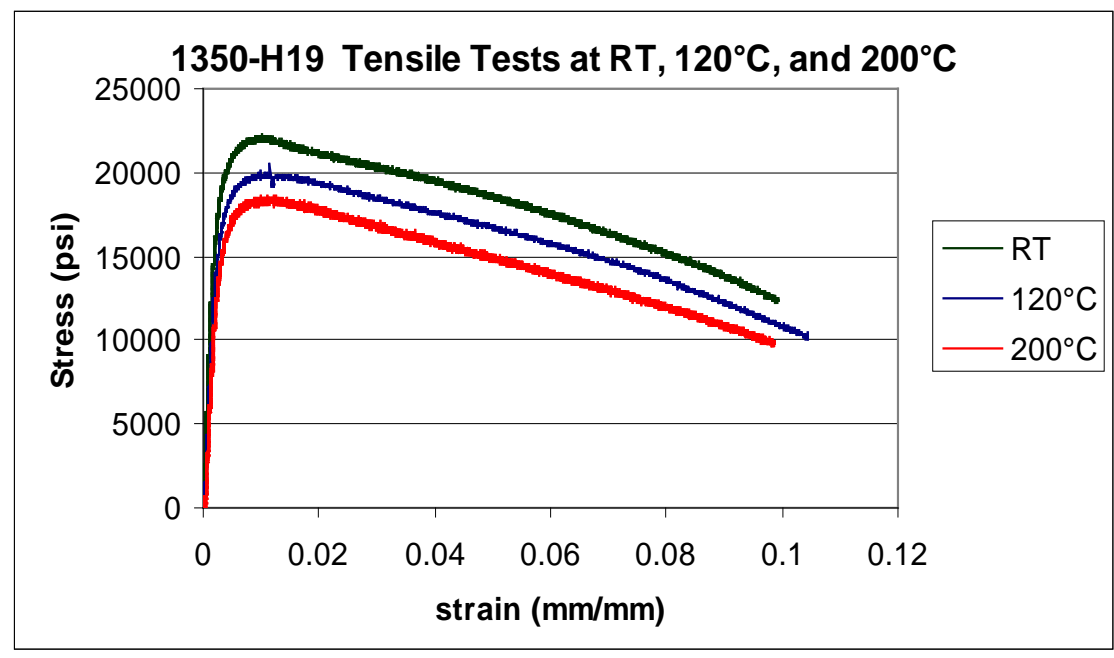

Figure 5-21 The tensile stress-strain curves for $1350-\mathrm{H} 19$ rod tested with $0.4 \% / \mathrm{min}$ strain rate loading.

The tensile test pair done at same temperature for $\mathrm{RT}, 120^{\circ} \mathrm{C}$ and $200^{\circ} \mathrm{C}$ are shown in Figure $5-22$, respectively. The self-consistency of the test pairs indicated the high quality of the test results and the repeatability of the tests. Figure 5-22, the conventional extensometer was used in Series 1 for deformation control, and the integrated device of the extensometer and Laser Mike was used in Series 2. Figure 5-22-room temperature test shows perfect match of the tensile stress-strain curves obtained from different deformation control devices. This further validated the reliability of our integrated extensometer/ Laser Mike device for deformation control and measurement. The integrated extensometer/Laser Mike device was used for high temperature testing, good agreement of the test results also shown in Figure 5-22. 


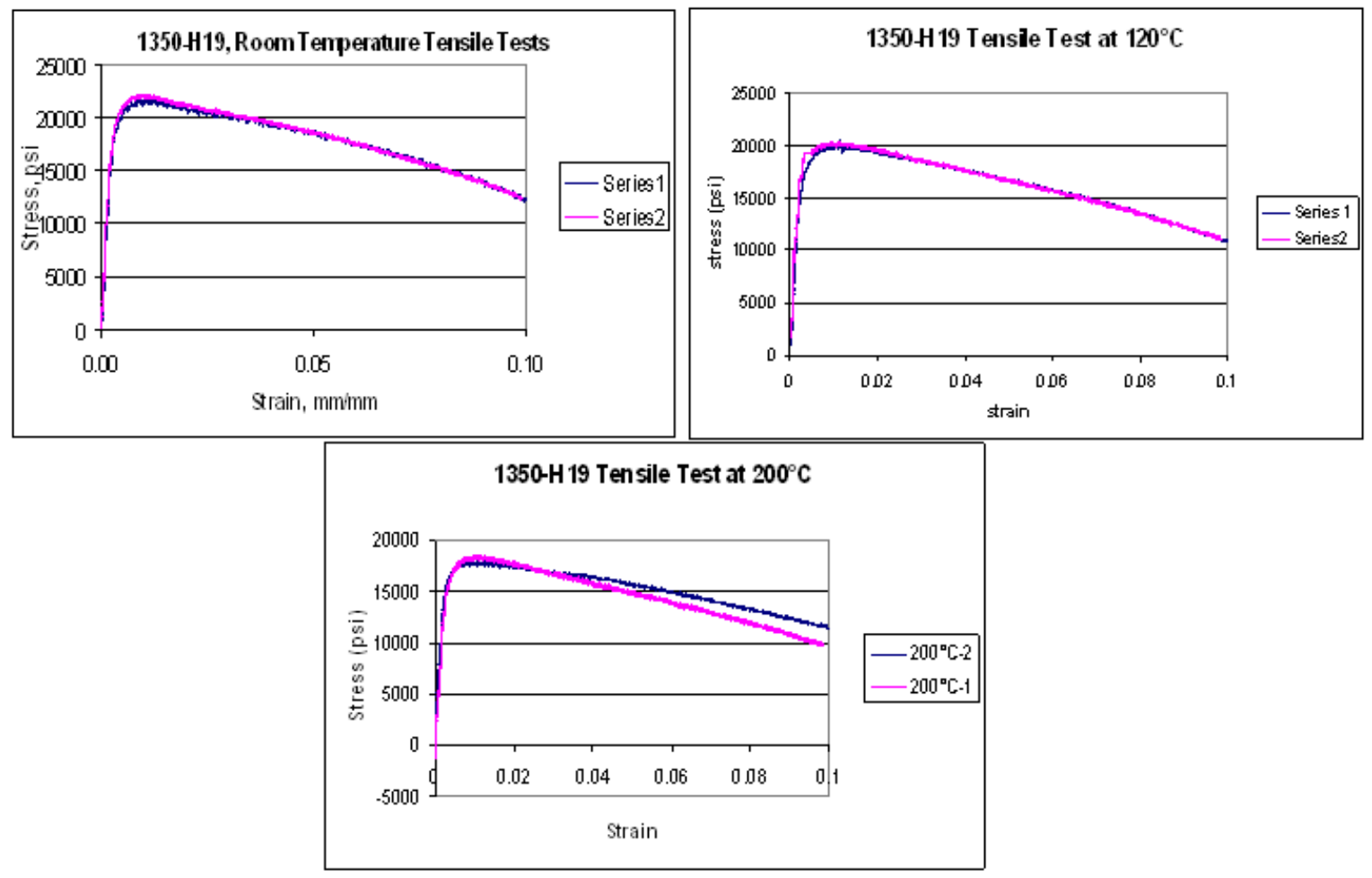

Figure $5-22$ Tensile test pairs done at $20^{\circ} \mathrm{C}, 120^{\circ} \mathrm{C}$, and $200^{\circ} \mathrm{C}$.

The impact of loading rate to the tensile strength of 1350-H19 at high temperature is shown in Figure 5-23, where the tensile strength increase accordingly with the increase of the loading rate. This phenomenon did not appear for room temperature tensile testing.

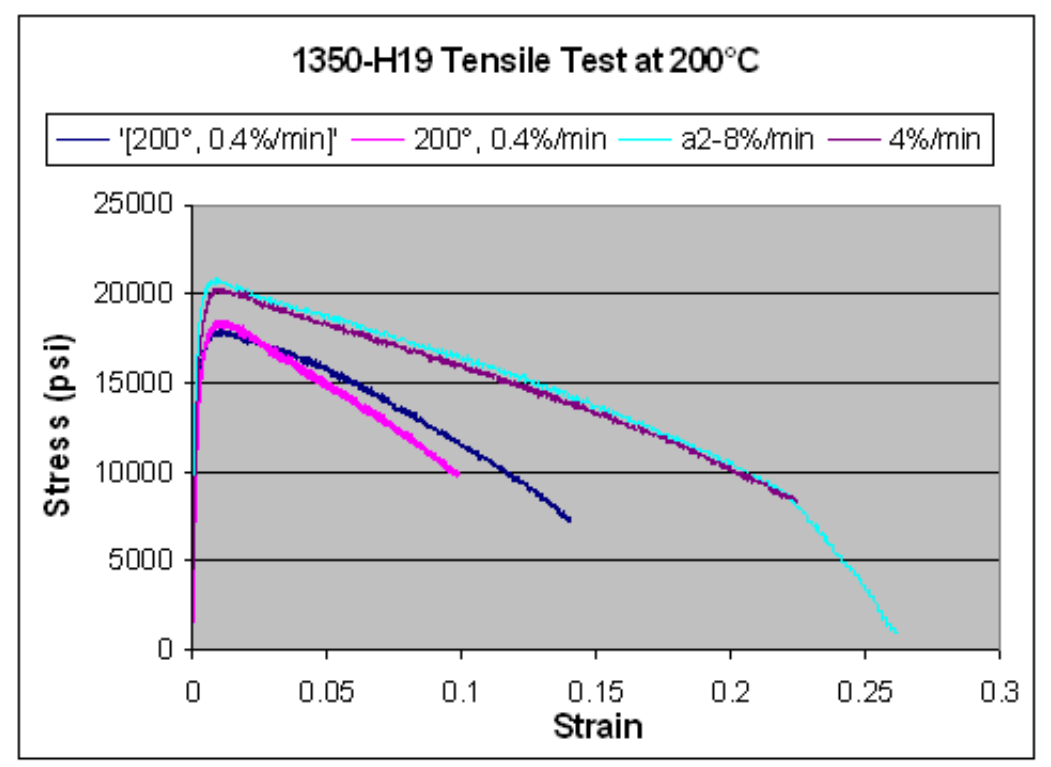

Figure 5-23 1350- $\mathrm{H} 19$ tensile tests done at $0.4 \% / \mathrm{min}, 4 \% / \mathrm{min}$, and $8 \% / \mathrm{min}$ strain rate loading. 


\subsection{Drake Conductor SCC System Mechanical Properties}

The received single-stage connector fitting baseline materials from TVA includes: (1) Baseline sleeve and the core-grip as shown in Figure 5-24 ; (2) crimped SCC, including the SCC and TSC fittings, shown in Figure 5-25, where the fitting at the top portion of the figure is a TSC fitting. One of the SCC fitting samples was cut into half, one piece was used for mechanical property evaluation and the other half was later irradiated in ORNL High Flux Isotope Reactor (HFIR) for in-situ compressive residual stress measurement evaluation.

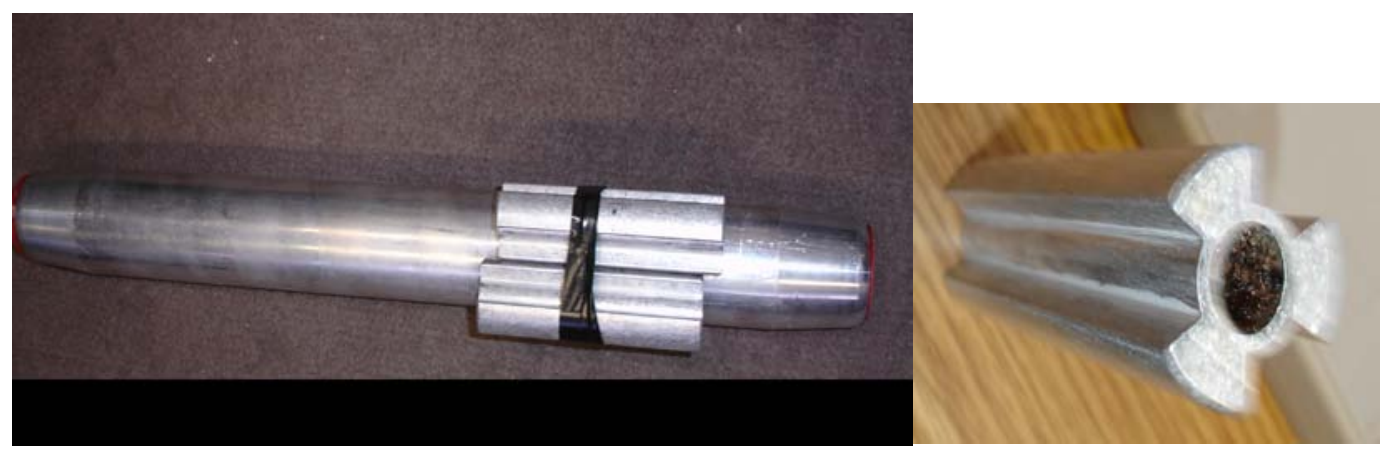

Figure 5-24 SSC fitting (left) and the associated core-grip (right).

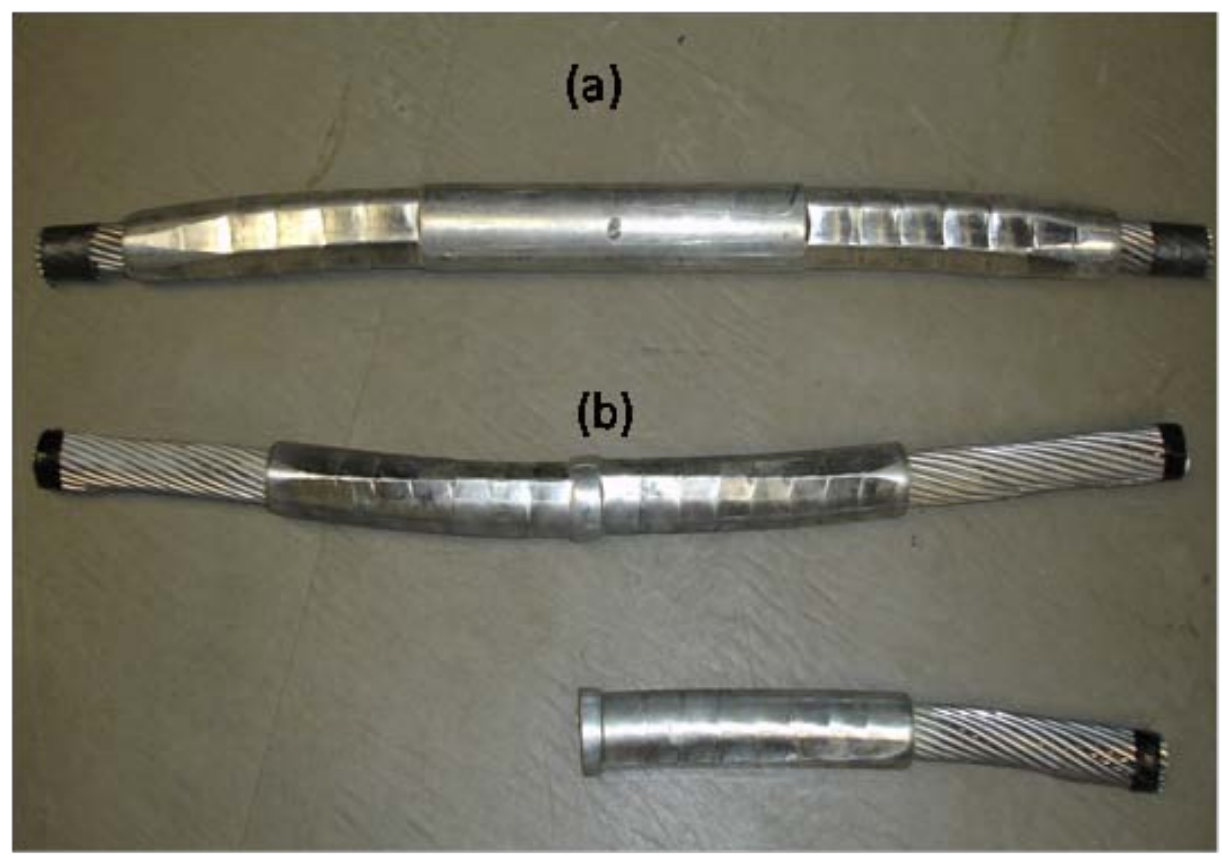

Figure 5-25 Crimped splice connector fittings: (a) TSC, and (b) SSC fitting; one SSC was cut into half that was used in neutron scattering experiments for residual stress measurements. 


\subsubsection{Specimen Preparation for SSC System Components}

Thermal-mechanical test specimens were directly machined from the baseline aluminum splice sleeve of 3003-H183 and the core-grip of 6061-T6, as illustrated in Figure 5-26.
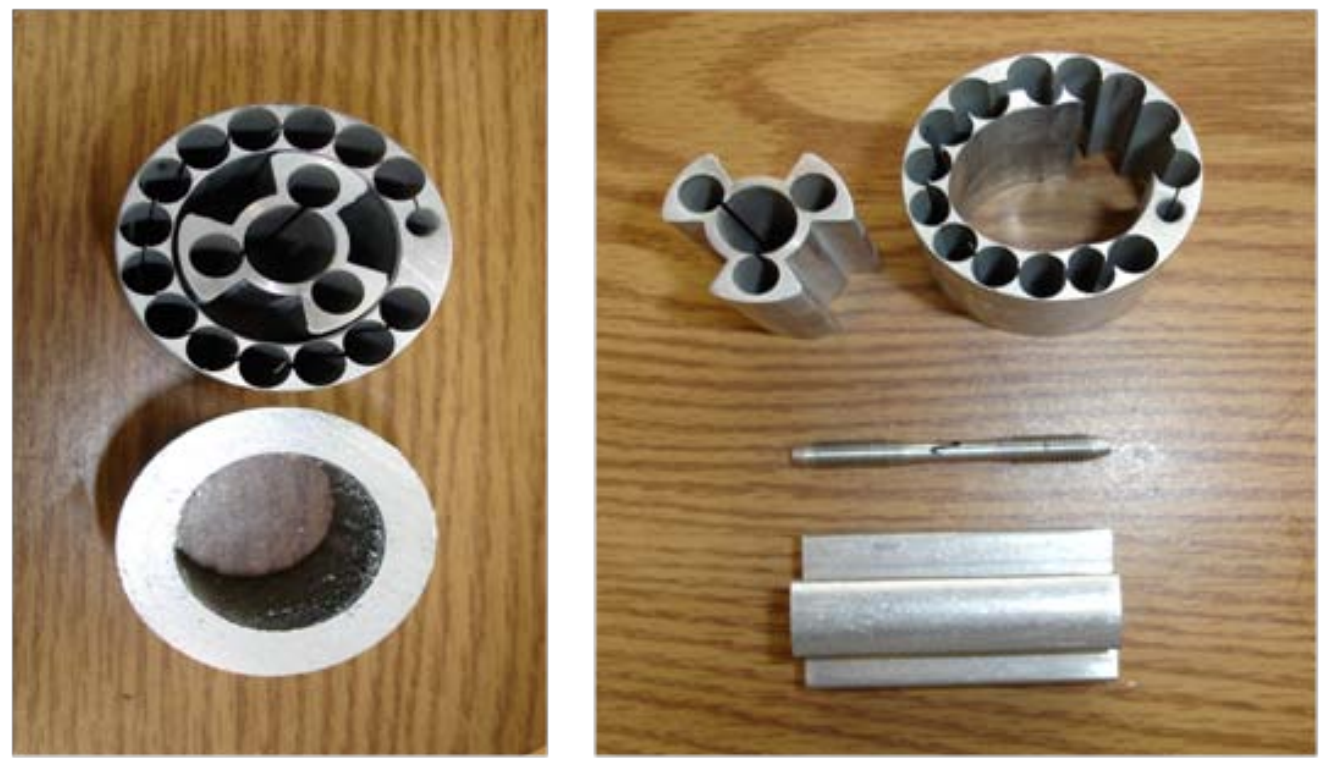

Figure 5-26 Tensile and fatigue samples for 3003-H183 and 6061-T6 materials were machined directly from the baseline splice sleeve and the associated core-grip.

\subsubsection{In-situ Mechanical Property Evaluation of ACSR SSC Fittings}

The in-situ mechanical testing was carried out on the received baselines and the crimped SSCs, This includes 3003-H183 aluminum from splice sleeve and 6061-T6 aluminum from the core grip. ABI indentation technique with a 0.030 inch diameter spherical indenter was used in the NDE mechanical property testing. The ABI in-situ test results are summarized in Table 5-2.

Table 5-2 Summary of ABI test results

\begin{tabular}{|l|l|l|l|l|}
\hline Test Samples & $\begin{array}{l}\text { Strength } \\
\text { Coeff., }[\mathrm{ksi}]\end{array}$ & $\begin{array}{l}\text { Strain } \\
\text { Hardening, } \mathrm{n}\end{array}$ & $\begin{array}{l}\text { Yield stress, } \\
{[\mathrm{ksi}]}\end{array}$ & $\begin{array}{l}\text { Tensile } \\
\text { Strength [ksi] }\end{array}$ \\
\hline Splice sleeve-1 & 40.8 & 0.091 & 23.1 & 30.0 \\
\hline Splice sleeve-2 & 42.5 & 0.086 & 24.9 & 31.5 \\
\hline Splice sleeve-3 & 44.8 & 0.091 & 25.4 & 32.9 \\
\hline Crimped sleeve-1 & 41.0 & 0.084 & 24.3 & 30.6 \\
\hline Crimped sleeve-2 & 41.7 & 0.083 & 24.9 & 31.1 \\
\hline Crimped sleeve-3 & 43.4 & 0.086 & 25.4 & 32.2 \\
\hline Core-grip-1 & 81.6 & 0.091 & 46.4 & 60.0 \\
\hline Core-grip-2 & 75.9 & 0.073 & 48.2 & 58.3 \\
\hline Core-grip-3 & 73.0 & 0.067 & 48.1 & 57.0 \\
\hline
\end{tabular}

It is interesting to note that there are no significant changes in yield stress at the surface between the baseline sleeve and the crimped sleeve. This was due to the elastic recovery at the surface of 
the crimped sleeve after die removal. This phenomenon was further verified from the FEM analysis. Based on these mechanical data, a detailed non-linear finite element analysis coupled with the "contact element" approach was carried out to investigate the deformation mechanism of a crimped SSC and to estimate the compressive residual stress distribution within a SCC. The residual stress field left within a splice connector right after the crimping event provides a baseline of the boundary condition for further lifetime estimates of SCC system during operation. In order to benchmark the in-situ residual stress evaluation of the received crimped SCC fitting, a neutron scattering technique for mapping residual stress within the SCC fitting was conducted at ORNL HFIR reactor facility.

\subsubsection{Tensile and Compression Test of 3003-H183 Aluminum Splice Sleeve}

The tensile tests of splice sleeve material, 3003-H183 are shown in Figure 5-27. Significant reduction of tensile strength was observed for test temperature greater than $200^{\circ} \mathrm{C}, \sim 28 \%$. Due to SSC compressive forming mechanisms, the compression tests at room temperature were also carried out for the splice sleeve material shown in Figure 5-28. It is interesting to note that the compression test results are more close to that of the ABI indentation test results. The yield transition of compression test curves are initiated earlier than that of tension test results. Similar results were shown in 1350-H19 materials, Figure 5-29. Thus, the flow stress trends used in finite element analyses were based on the compression tests results.

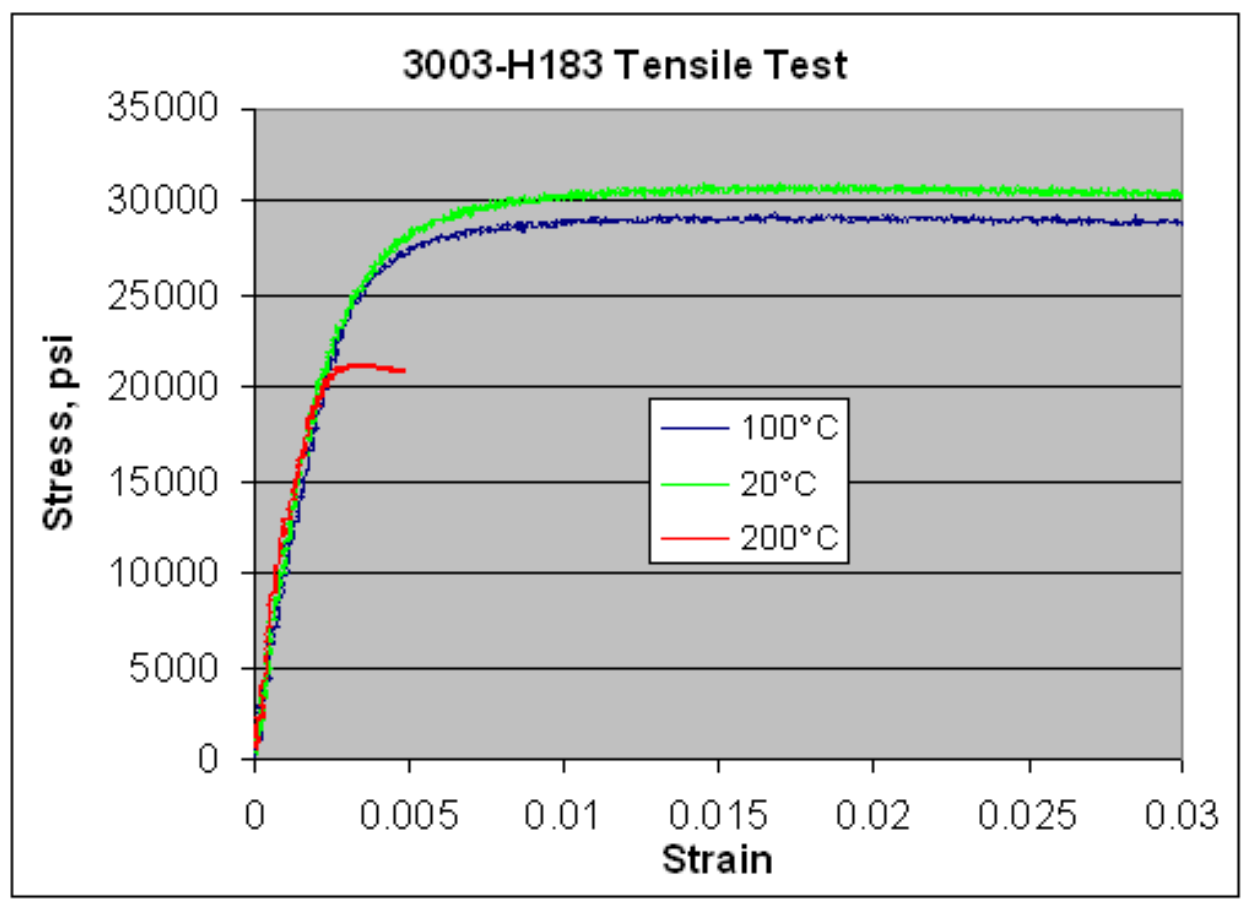

Figure 5-27 Tensile test results of 3003-H183 aluminum. 


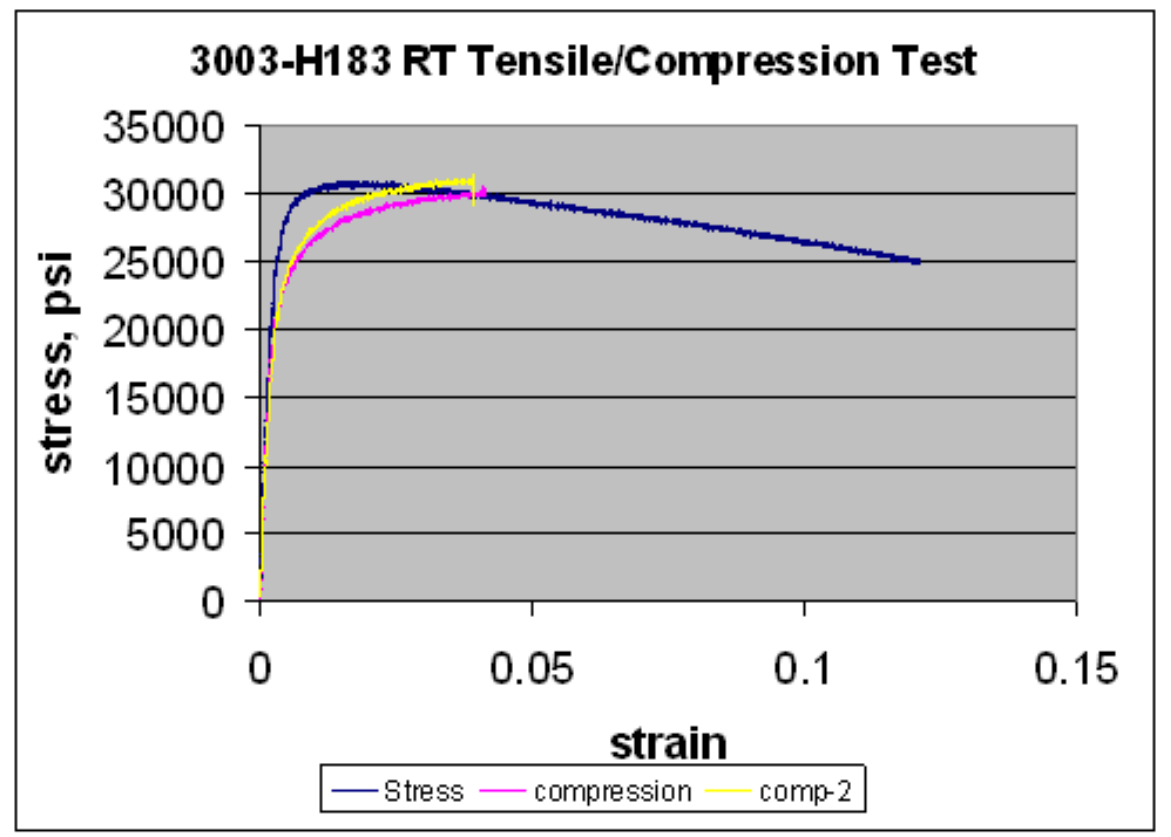

Figure 5-28 Comparison of tensile/compression trend curves for 3003-H183.

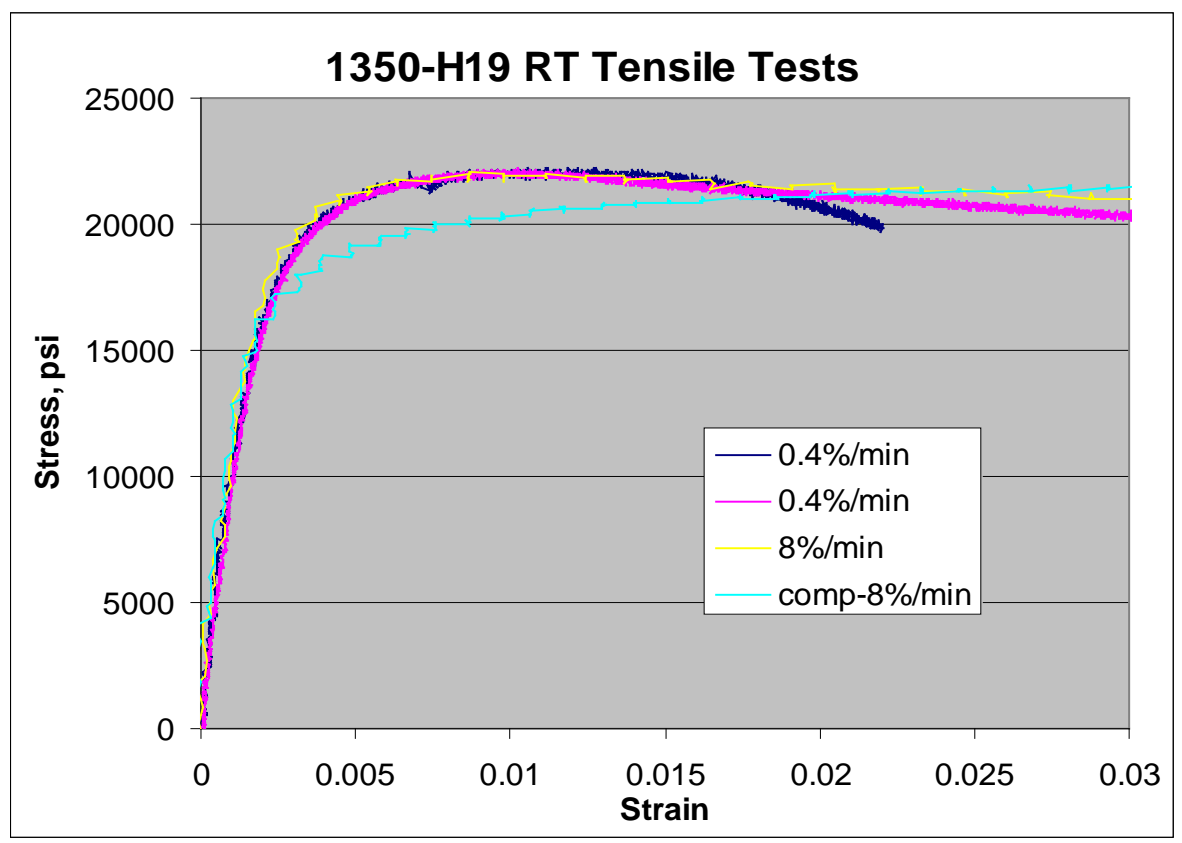

Figure 5-29 Comparison of tensile/compression trend curves for 1350-H19 


\subsubsection{Tensile Test of 6061-T6 Aluminum Core-grip}

The tensile tests of core-grip material, 6061-T6 are shown in Figure 5-30. Significant reduction of tensile strength was also observed for test temperature greater than $200^{\circ} \mathrm{C}, \sim 23 \%$.

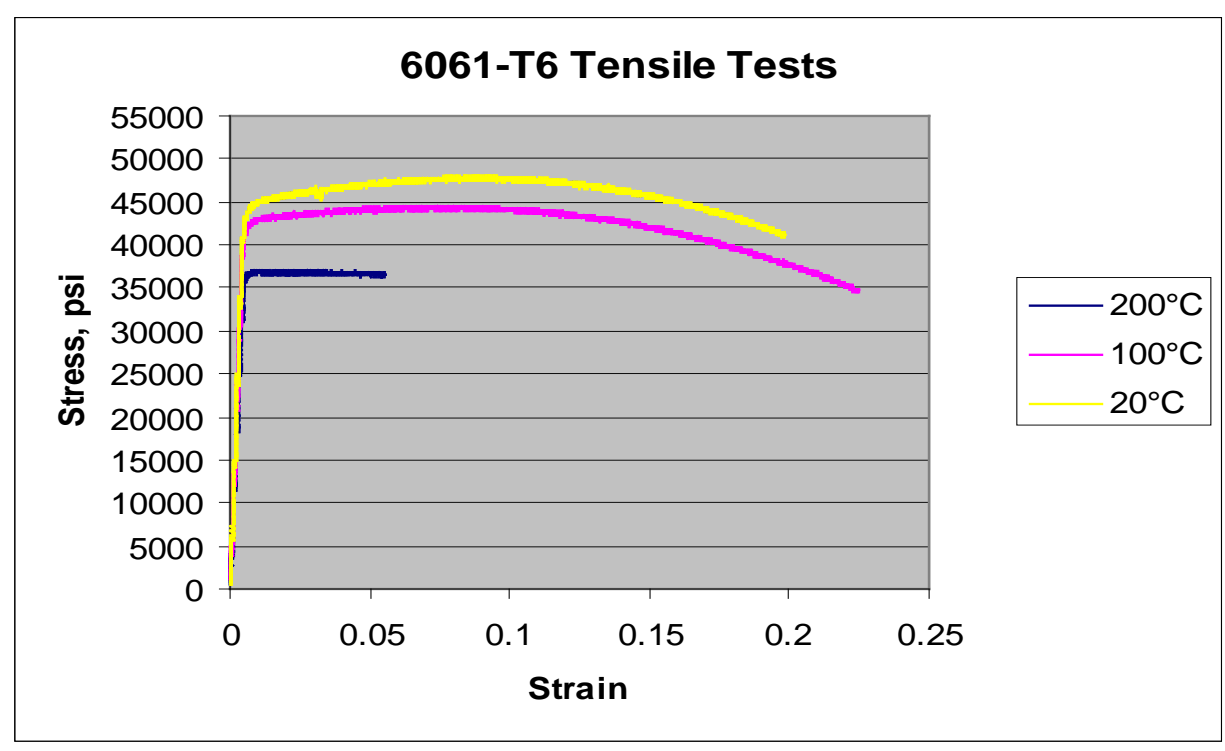

Figure 5-30 Tensile test results of 6061-T6 aluminum

\subsection{Material Flow Properties Developed for Finite Element Analyses}

The thermal mechanical tests include tensile, creep, fatigue testing and thermal-mechanical fatigue. These tests were carried out for 1350-H19 (conductor), 3003-H183 (sleeve) and the 6061-T6 (core-grip). The details of the test results are stated in Appendix C. Pertinent test information and results are summarized below.

- Significant decrease in strength at high temperatures, for instance, at $260^{\circ} \mathrm{C}$, the strength reductions are

o 1350-H19: 77\%;

o 3003-H183: 67\%

o 6061-T6: 45\%; w/ 10 hour holding: 60\%.

- In order to simulate 2 thermal cycles per day of power line environment, the holding time for the tensile samples at the target temperature was set to 10 hours.

- Thermal annealing has significant impact on the SSC component strength, as revealed from the decrease in tensile strength of annealed samples at room temperature, especially for annealing temperature greater than $200^{\circ} \mathrm{C}$. The considered effective annealing temperature ranges for the SSC components were set between $200^{\circ} \mathrm{C}$ to $370^{\circ} \mathrm{C}$ with a duration of 10 hours.

- The true stress-true plastic strain curves were generated through thermal mechanical testing for 1350-H19, 3003-H183 and 6061-T6 aluminum materials, to provide inelastic mechanical properties input for the FEM analyses. The baseline and annealed data of $260^{\circ} \mathrm{C} / 10$-hour 
holding are presented in the Figure 5-31. The annealed data, shown in the right column of Figure 5-31, shows a significant decrease in the yield and the associated stress-strain curves compared to that of baseline data shown in the left column.
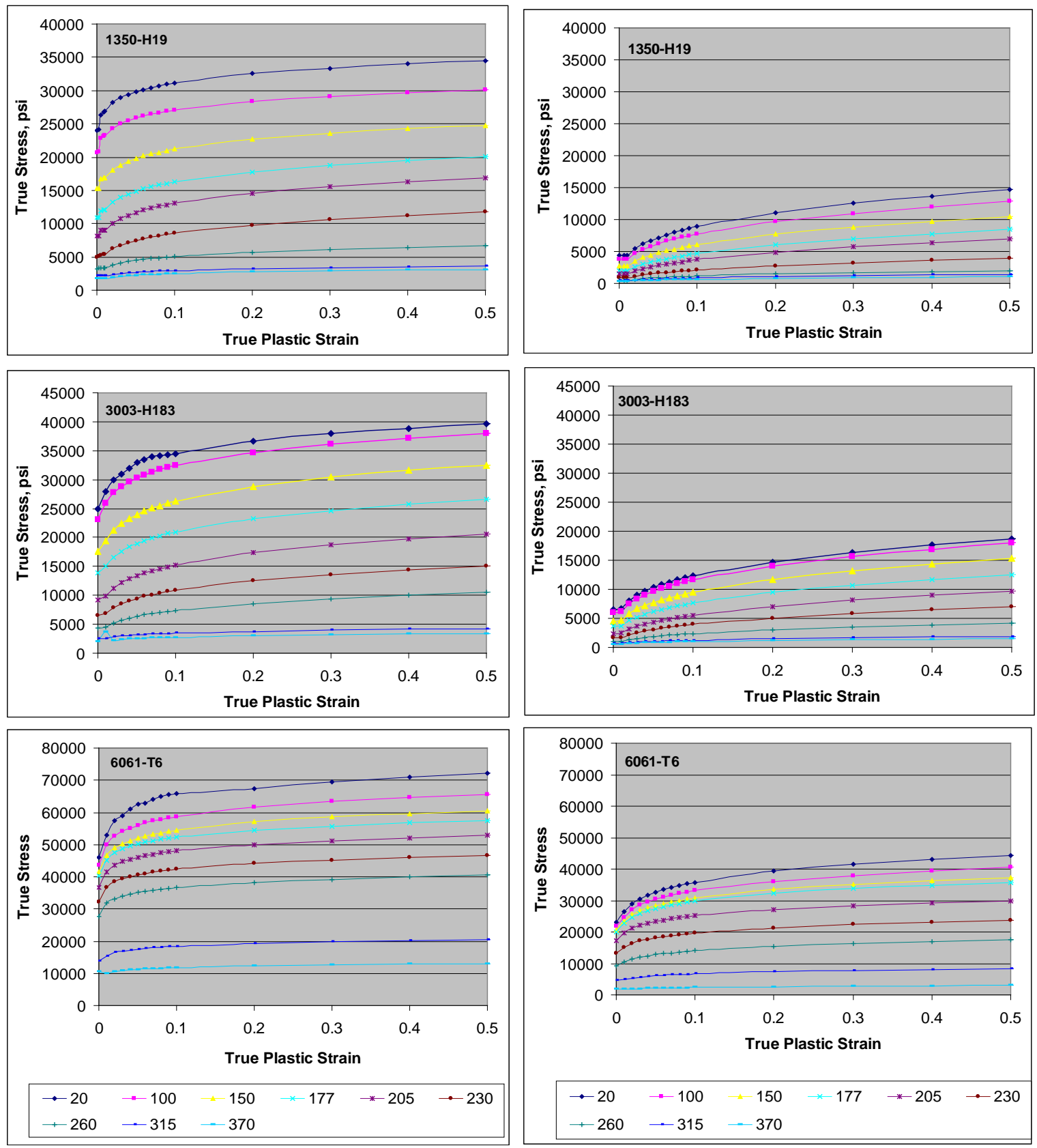

Figure 5-31 True stress vs. true plastic strain curves for (top) 1350-H19, (middle) 3003-H183, and (bottom) 6061-T6 aluminums; Left column is the baseline data and the right column is the data from the annealed samples, with $260^{\circ} \mathrm{C}$ annealing temperature at a duration of 10 hours. 


\subsection{Creep Test Results}

The creep tests for 1350-H19 aluminum were carried out on the cantilever and pneumatic creep testers. Test temperatures were in the range from $25^{\circ} \mathrm{C}$ to $250^{\circ} \mathrm{C}$, and the tension loading ranged from $2.5 \mathrm{ksi}$ to $17.7 \mathrm{ksi}$ (60-lb to 450-lb). Significant high creep rates were observed for the test temperature range above $150^{\circ} \mathrm{C}$ and load range greater than $6 \mathrm{ksi}(150-\mathrm{lb})$. It is interesting to note that for the creep test of $5.0 \mathrm{ksi}(120-\mathrm{lb})$ loading and with initial test temperature at $120^{\circ} \mathrm{C}$, marginal increase in creep strain was observed up to 35,000 min. However, due to temperature controller malfunction after 35,000 minutes, the test temperatures started to drift between $120^{\circ} \mathrm{C}$ and $240^{\circ} \mathrm{C}$. This unexpected thermal cycling event results in a significant increase in the creep strain as shown in Figure 5-32.

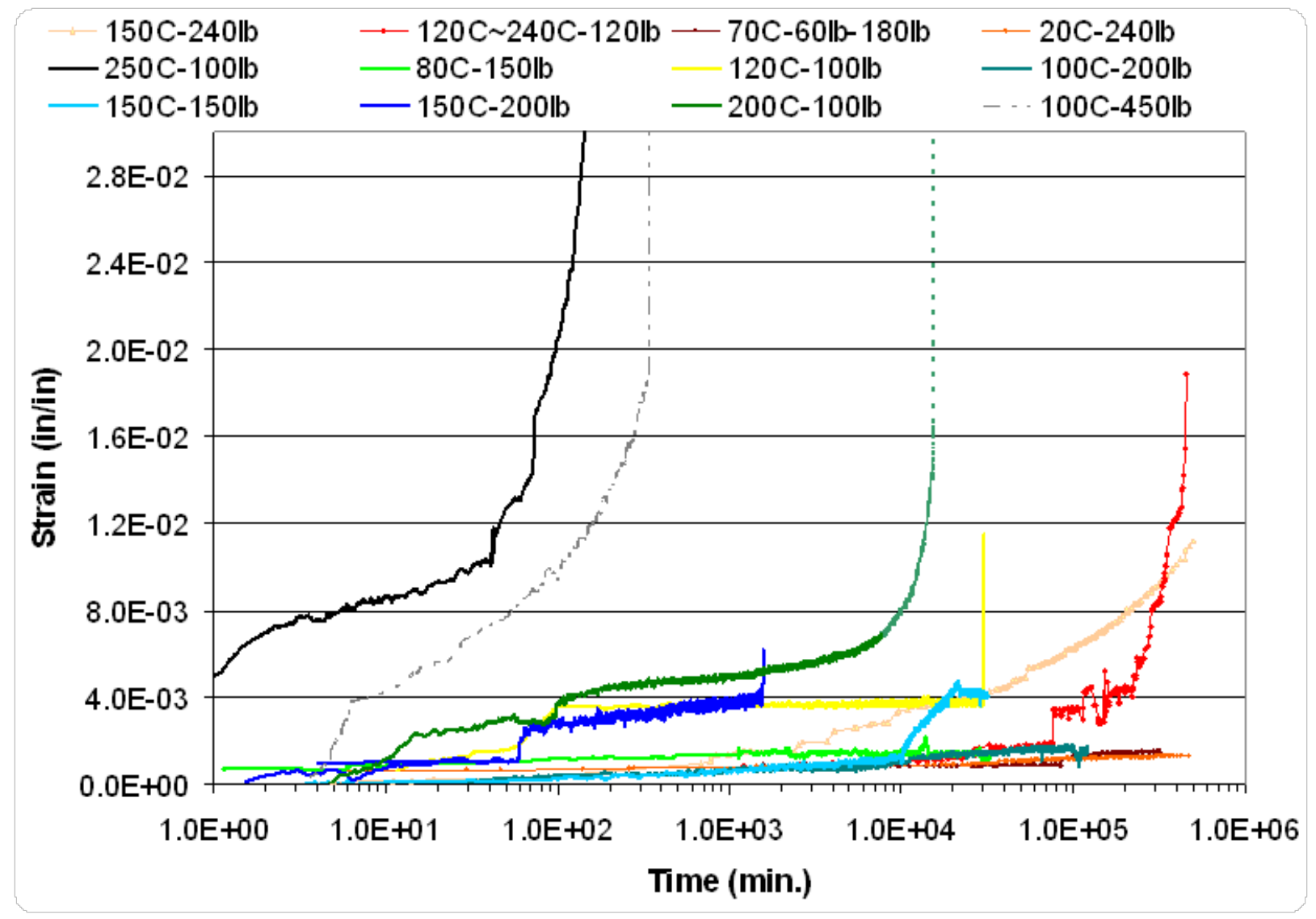

Figure 5-32 Creep test results for 1350-H19 aluminum with gage diameter of 0.18-in. For the creep test carried out at $70^{\circ} \mathrm{C}$, initial applied load was $60-\mathrm{lb}$ and after 8,000 min $180-\mathrm{lb}$ was applied to the creep test. 


\subsection{Isothermal Fatigue Test Results}

The fatigue test results indicate that the effective fatigue life of the SSC system materials is strongly dependent on the temperature and strain amplitude. Different aging responses were observed for room temperature and high temperature fatigue tests, as illustrated in Figure 5-33 and Figure 5-34, respectively, for 1350-H19. Similar behaviors were also observed for 3003H183 and 6061-T6.

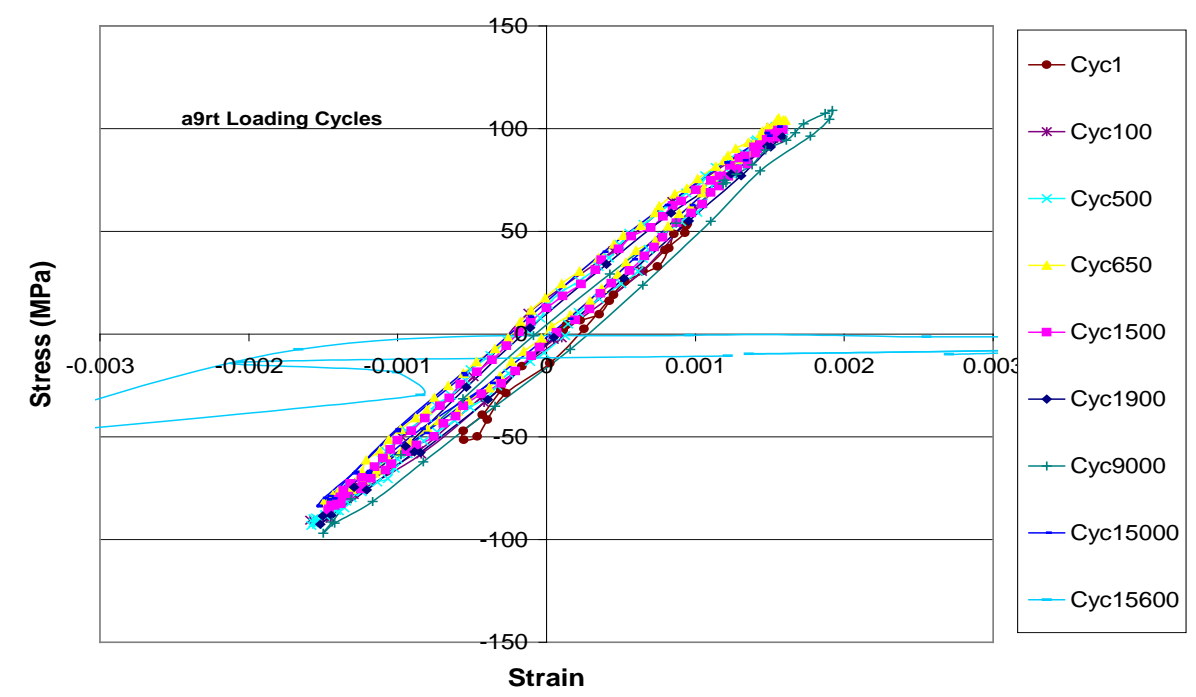

Figure 5-33 Room temperature fatigue test with $0.2 \%$ strain cycle, the specimen failed after reaching 15,600 cycles.

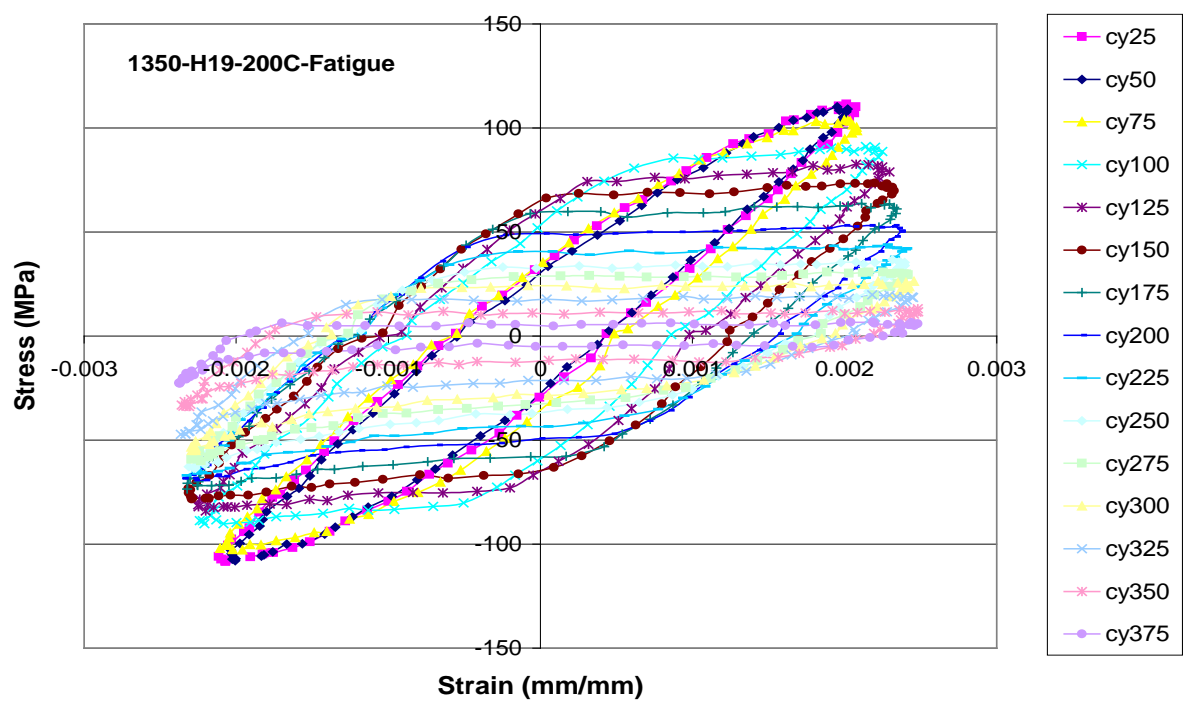

Figure 5-34 Fatigue test carried out at $200^{\circ} \mathrm{C}$ with $0.25 \%$ strain cycle. The material shows pronounced softening and permanent deformation after about 150 cycles, and the sample material fails to carry stress after about 350 cycles. 


\subsection{Thermal-Mechanical Fatigue Test Results}

Under thermal-mechanical fatigue (TMF) testing, the total strain is maintained near $0 \%$ and temperature cycles between $75^{\circ} \mathrm{C}$ and $175^{\circ} \mathrm{C}$. The TMF testing results indicate that the associated material hardening induced by thermal cycling seems to be annealed by the testing temperatures during the alternating aging cycles and no obvious long term TMF damage or fracture was observed from tested samples after some 9000 TMF cycles. In order to simulate the tension loading drop of a power line at heat-up cycle, a series of TMF testes were also carried out with variable "total strain" cycle ranges from $0 \%$ to $0.2 \%$ or $0.3 \%$. No long term aging degradation was observed from this type of TMF tests. The TMF test data from 1350-H19 samples for 0\% total strain and the variable total strain are shown in Figure 5-35 and Figure 5-36, respectively. Similar material responses were also observed for 3003-h183, and 6061-T6 materials.

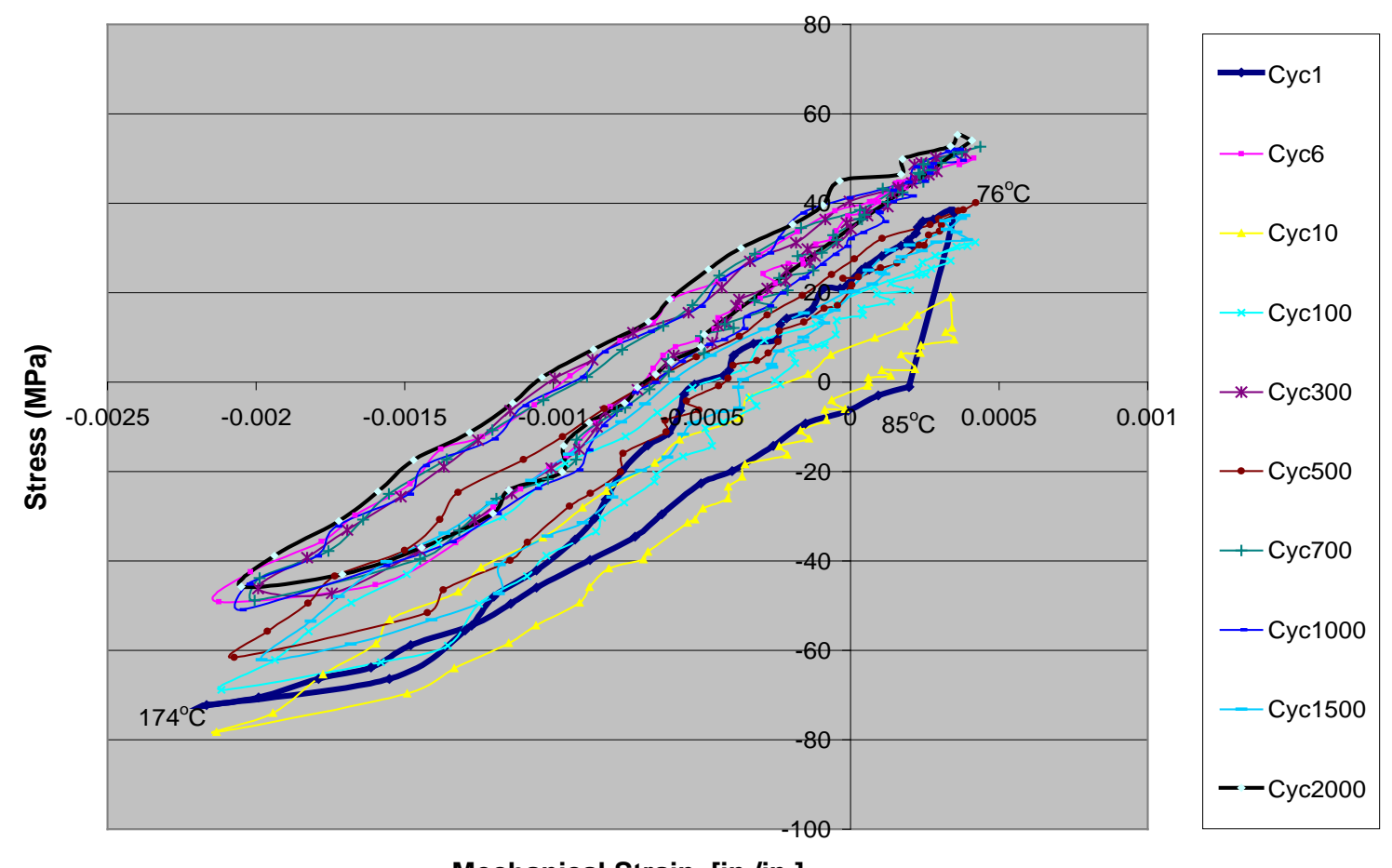

Mechanical Strain, [in./in.]

Figure 5-35 1350H-19 TMF test with total strain maintained near $0 \%$ and temperature cycle range of $75^{\circ} \mathrm{C}$ to $175^{\circ} \mathrm{C}$. The material softening and hardening appeared in alternative TMF cycles seem to balance each others and no permanent aging damage was observed. 


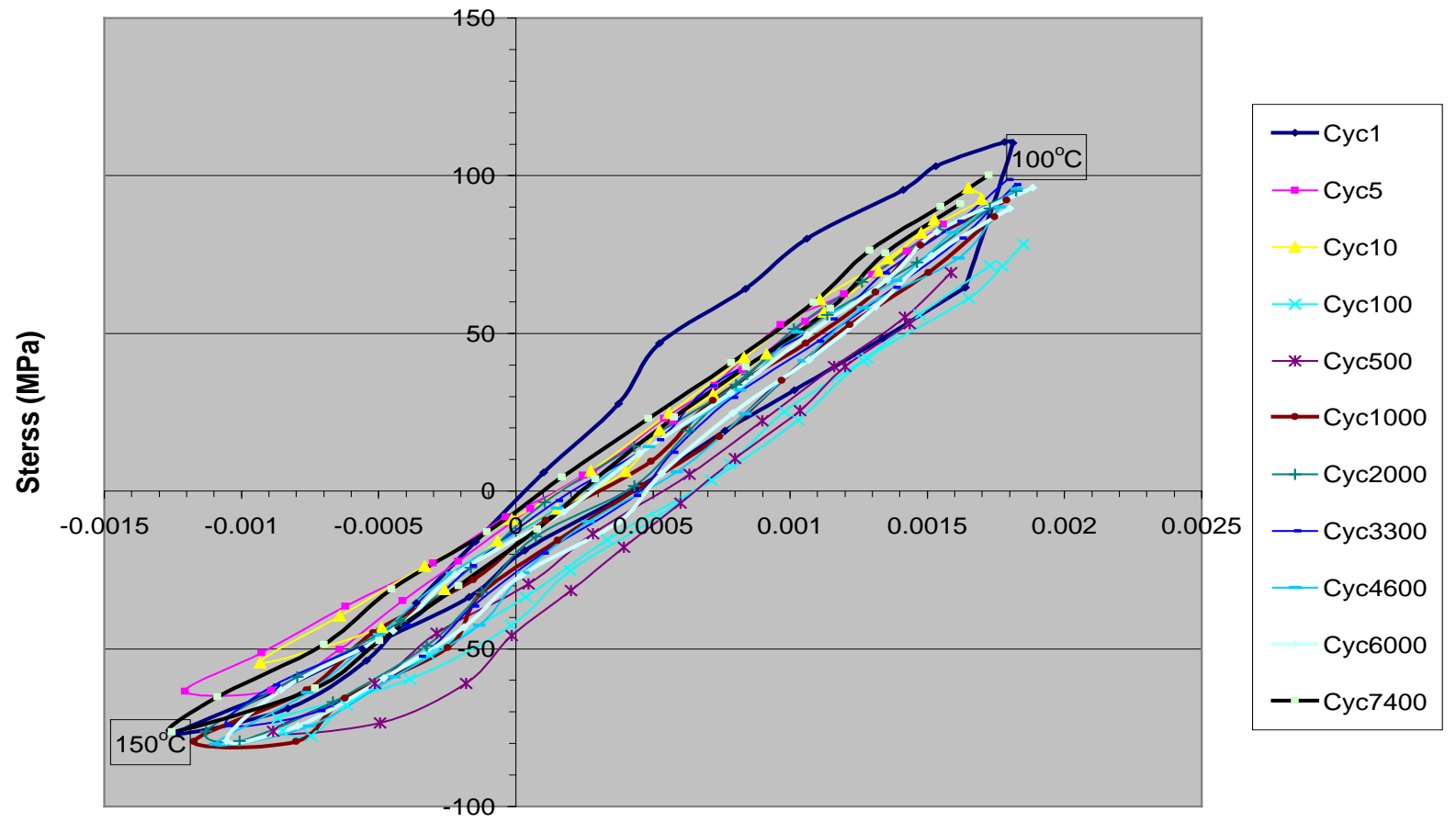

Mechanical Strain, in./in.

Figure 5-36 $1350 \mathrm{H}-19$ TMF test with total strain cycling between $0 \%$ to $0.2 \%$ and temperature cycle range of $100^{\circ} \mathrm{C}$ to $150^{\circ} \mathrm{C}$. No significant aging was observed from the TMF data.

\subsection{SEM Microstructure Examinations}

SEM postmortem examinations of the fatigue samples obtained from SSC component materials reveals typical striation representative of fatigue fracture with the inhomogeneous textures having originated from the cold drawing mechanism, as illustrated in Figure 5-37. The SEM images of fractured 1350-H19 samples are also shown in Figure 5-38.

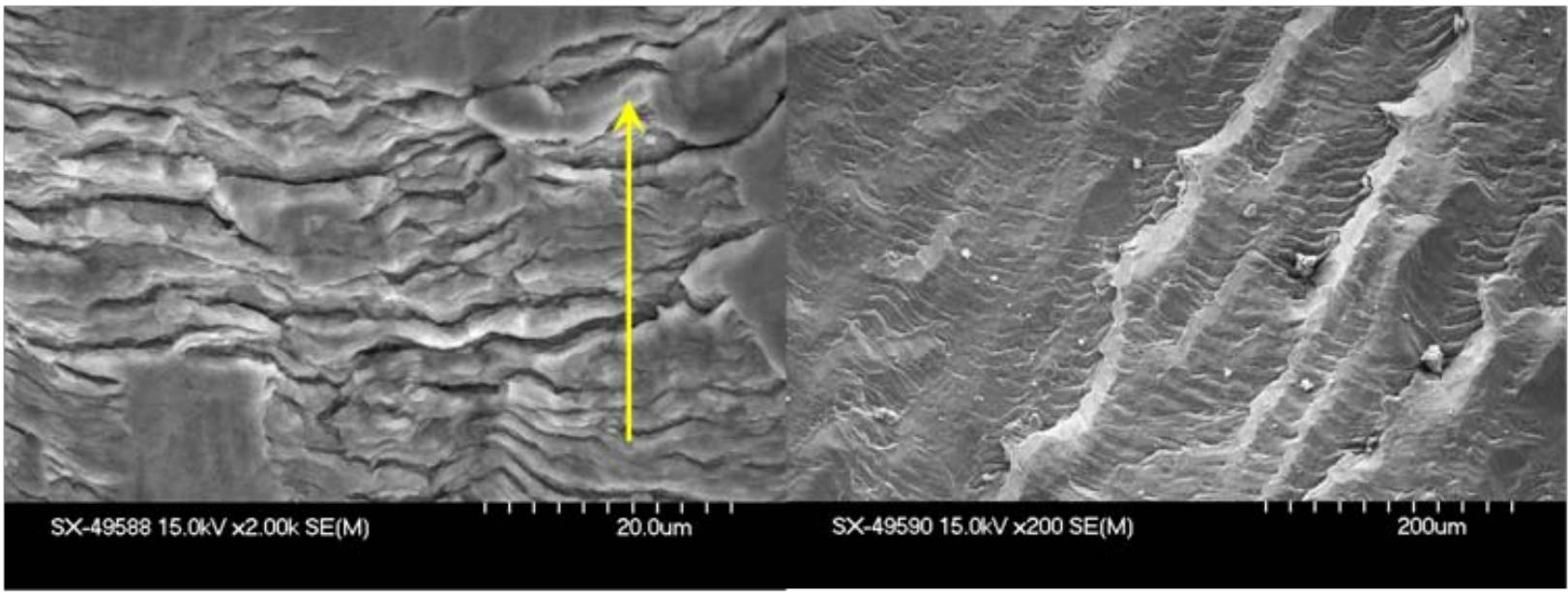

Figure 5-37 Fatigue fracture samples, with typical striation formation, yellow arrow indicates the orientation of the fatigue crack growth. 

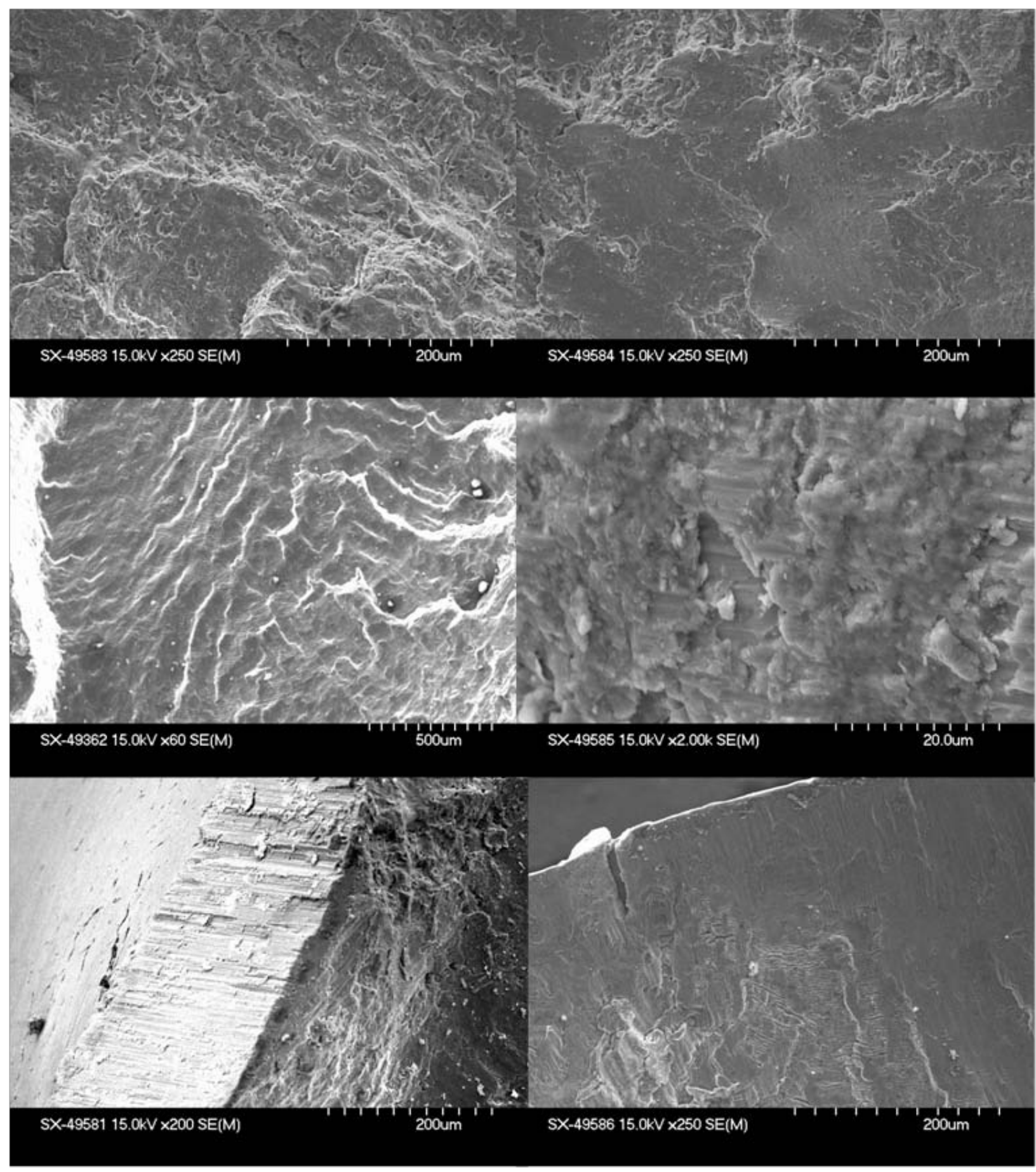

Figure 5-38 SEM images of typical 1350-H19 fatigue fractured samples. 


\subsection{NDE Impedance Measurements}

A Solartron 1260 Frequency Response Analyzer (FRA) was used to measure the impedance spectra from both an as-received baseline splice connector that had never seen service and a joint that had been used for 3 years. Both electrodes were connected to one aluminum wire on either side of the connector. Frequency was swept from $0.1 \mathrm{~Hz}$ to $100 \mathrm{kHz}$ with $10 \mathrm{mVAC}$ amplitude and 0 VDC bias. The resulting spectra are shown in Figure 5-39. The curves were modeled using a resistor and an inductor in series. The baseline resistance from the as-received joint was $0.0117 \Omega$. The used joint had a resistance of $0.0294 \Omega$, almost three times the baseline. This result shows that the aging connector following service for 600 cycles degrades its electrical performance.

Nevertheless, this type measurement is strongly depended on the path chosen for measuring impedance. We also tried of using circular clamp to secure the probe onto the conductor, instead of only using single conductor wire for sampling. In this approach, the difference of the measured impedance between the aged (600 cycles) and baseline conductors is negligible. This is probably due to the circular clamp provide the least resistance path for measuring impedance. Therefore, a significant number of tests for impedance measurement need to be done to provide systematic trend of aging induced impedance change in the connector system.

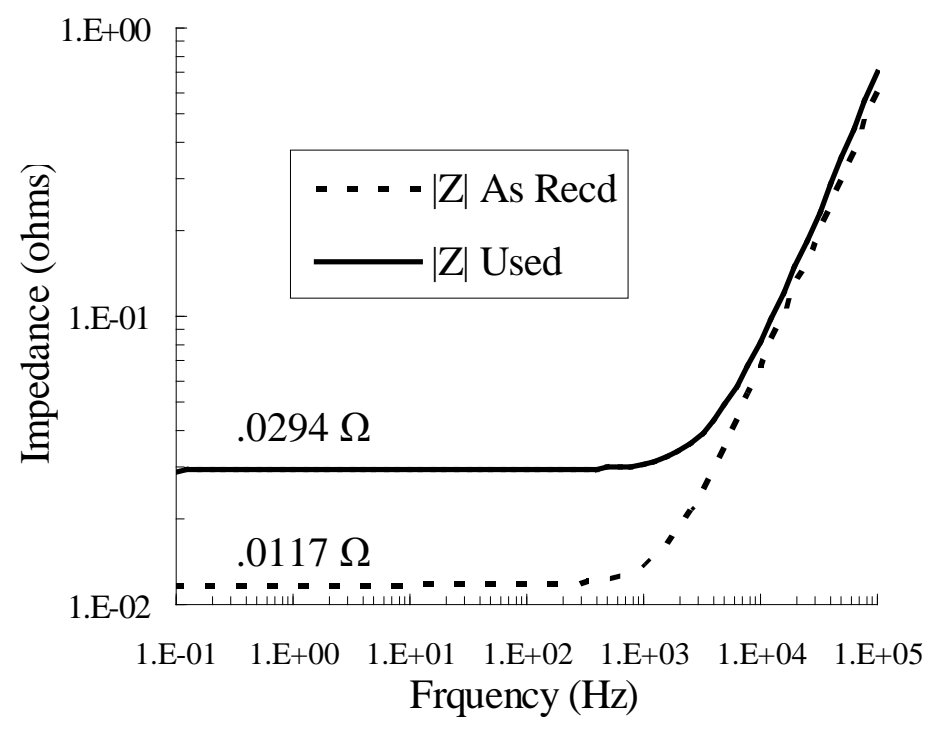

Figure 5-39 The impedance spectra from baseline and the aging splice connectors. 



\section{$\boldsymbol{6}$ INTEGRITY OF ACSR SINGLE-STAGE SPLICE CONNECTOR SYSTEM \& ITS FORMING MECHANISM}

The majority of overhead transmission lines currently use steel-reinforced core aluminum conductors (ACSR). The crimped type splice connectors are widely used in the power industry, including the two-stage splice connector (TSC) and the single-stage splice connector (SSC). However, the potential of failure frequency of SSC fitting at higher temperature operations is considered to be higher than that of TSC fitting. The main reason for the decreased durability of a SSC compared to that of a TSC is due to material mismatch of steel and aluminum in a SSC system. An aluminum core-grip is used for clamping and connecting the steel cores in a SSC, whereas for TSC a steel fitting tube is used to connect the steel cores. During thermal cycles, the thermal expansion/contraction at the aluminum core-grip is expected to be larger than that of the steel core in a SSC. Furthermore, in a TSC, the portion of the splice sleeve that contains the steel sleeve is not crimped. This is contrast to forming a SSC at the core-grip section. Thus, it is expected that there will be more plastic deformation and texture variation in the crimped splice sleeve at the core-grip section for a SSC. The consequence of this is the increase in resistivity of the splice sleeve and the increase of steady-state temperature for a SSC fitting compared to that of a TSC fitting, for a designated conductor operating temperature. Furthermore, the aluminum core-grip of SSC fitting has higher electric conductance compared to that of the steel sleeve of a two stage connector fitting; thus, the Joule heating induced temperature increase in the aluminum core-grip will be higher than that of the steel sleeve in a TSC. The above mentioned factors of temperature increase in SSC provide the recipe to further soften the aluminum core-grip and results in accelerated reduction of the clamping pressure or compressive stress between the aluminum core-grip and the steel core. Ultimately this will result in the final separation (or pull out) of the steel cores and aluminum conductor from the splice connector.

The root cause of accelerated aging that causes failures is generally attributed to poor installation or erroneous materials entering the manufacturing process. For example, when full tension splice connector fittings that do not have adequate crimping pressure or have too short a length of conductor pushed into the connector sleeve prior to crimping, they will overheat and cause accelerated degradation and this leads to failure. Introducing higher operating temperatures, whether cyclic or steady state will accentuate weaknesses in the components and lead to incremental failures. Furthermore, annealed strands with an increased creep rate will allow them to flow away from the splice connector rather than have a residual stress against the splice connector. This situation is further worsened with thermal expansion/contraction cycles when the conductor loading varies. Moreover, for a splice connector joint, the joint resistance does not remain constant but will increase during operating time. This long-term behavior of joint resistance can be influenced by different aging mechanisms, like compressive stress relaxation, oxide layer built-up, corrosion processes, inter-diffusion, electromigration, and fretting.

The research project focused on the SSC system because SSC fitting appeared to be the potential weakest link in the current power transmission system. To gain a more clear understanding of the 
SSC designs, a unique computer simulation protocol has been developed at Oak Ridge National Laboratory (ORNL) that can be used to identify the potential failure mechanisms and to evaluate the long term reliability issues. These results clearly demonstrate that large plastic deformations are experienced by the connector materials during the forming/crimping process. Such a large deformation is necessary to form the large compressive residual stress fields within the connector system. The residual compressive stress remaining in the splice connector after forming is a key parameter, since it provides the initial clamping strength to confine the conductor in a connector system. If the residual stress is not sufficiently high, the conductor system will ultimately fail during operation.

\subsection{ANSI Standard for Splice Connector Integrity}

ANSI C119.4 specifies current cycle and mechanical tests for establishing a basis of performance for electrical connectors used to join aluminum-to-aluminum or aluminum-to-copper bare overhead conductors. This standard provides well-defined, reproducible requirements for electrical connectors and assures the user that connectors meeting these requirements will perform in a satisfactory manner when properly installed. Mechanical testing of ANSI C119.4 consists of pullout strength tests to compare the connector's results with the rated conductor strength per applicable ASTM conductor standards. Three classifications result from the mechanical testing: (1) Class 1 , full tension $=95 \%$ of the rated conductor strength of the conductor being joined, (2) Class 2, partial tension $=40 \%$ of the rated conductor strength of the conductor being joined, and (3) Class 3 , minimum tension $=5 \%$ of the rated conductor strength of the conductor being joined.

For a full tension splice connector fitting, the compressive residual stress fields within a crimped splice connector provide an initial binding strength of the splice connector to secure the aluminum conductor. The inherited forming mechanism induced accelerated aging of the crimped splice connectors can be attributed to material creep, thermal-mechanical cycle fatigue, aluminum oxide film build-up and other microstructure evolution at the interfaces within the connector system. Therefore, it is expected that significant degradation of this initial residual stress will occur during the service, especially at higher operation temperature. The consequence of degradation of the initial compressive stress field is the reduction of connector initial rate tensile strength that was evaluated according to ANSI C119.4. Therefore, the compressive residual stress within a compressive connector can serve as a good primary index that can assist in the prediction of the remaining life of a conductor system during service.

In order to develop a realistic lifetime prediction model for the connector, detailed knowledge and understanding of the deformation mechanism that dictates the compressive stress distribution are needed. In this aspect, two research efforts were carried: (1) using finite element analysis to simulate the compressive stress distribution within a clamped SSC at the core grip section and conductor section, (2) in-situ nondestructive evaluation of using neutron scattering technique to benchmark the residual stress profile within the new SSC fitting received form TVA. 


\subsection{Analytical Evaluation of Single-Stage Splice Connector Forming}

\subsubsection{Descriptions of ACSR Drake SSC System and Its Forming Procedures}

The Drake conductor, consisting of 26 aluminum conductor wires and 7 core steel wires, the ALCOA aluminum splice sleeve and the core-grip, and the associated 60 tons 6012CD die set were used in the finite element modeling (FEM) to simulate the splice connector forming process and to determine the compressive residual stress fields. The detailed examination of the ALCOA 6012CD die indicates that the inner contour of the 6012CD die is neither elliptical nor circular geometry. The die cross-section contour is the combination of center Arc section of $2 \mathrm{~cm}$ radius and two straight line sections toward the ends of die. The die-set maximum opening distance is about $4.5 \mathrm{~cm}$. The geometry of the die-set and the associated details needed for FEM analyses are shown in Figure 6-1. A 60-ton hydraulic press used for forming SSC is controlled by a pressure switch set to switch off the hydraulic pressure when the hydraulic ram is at 60 tons (the maximum press pressure is $10,000 \mathrm{psi}$ ). Fittings are pressed until the two dies contact each other and can advance no further (causing the hydraulic pressure at the switch to escalate quickly).
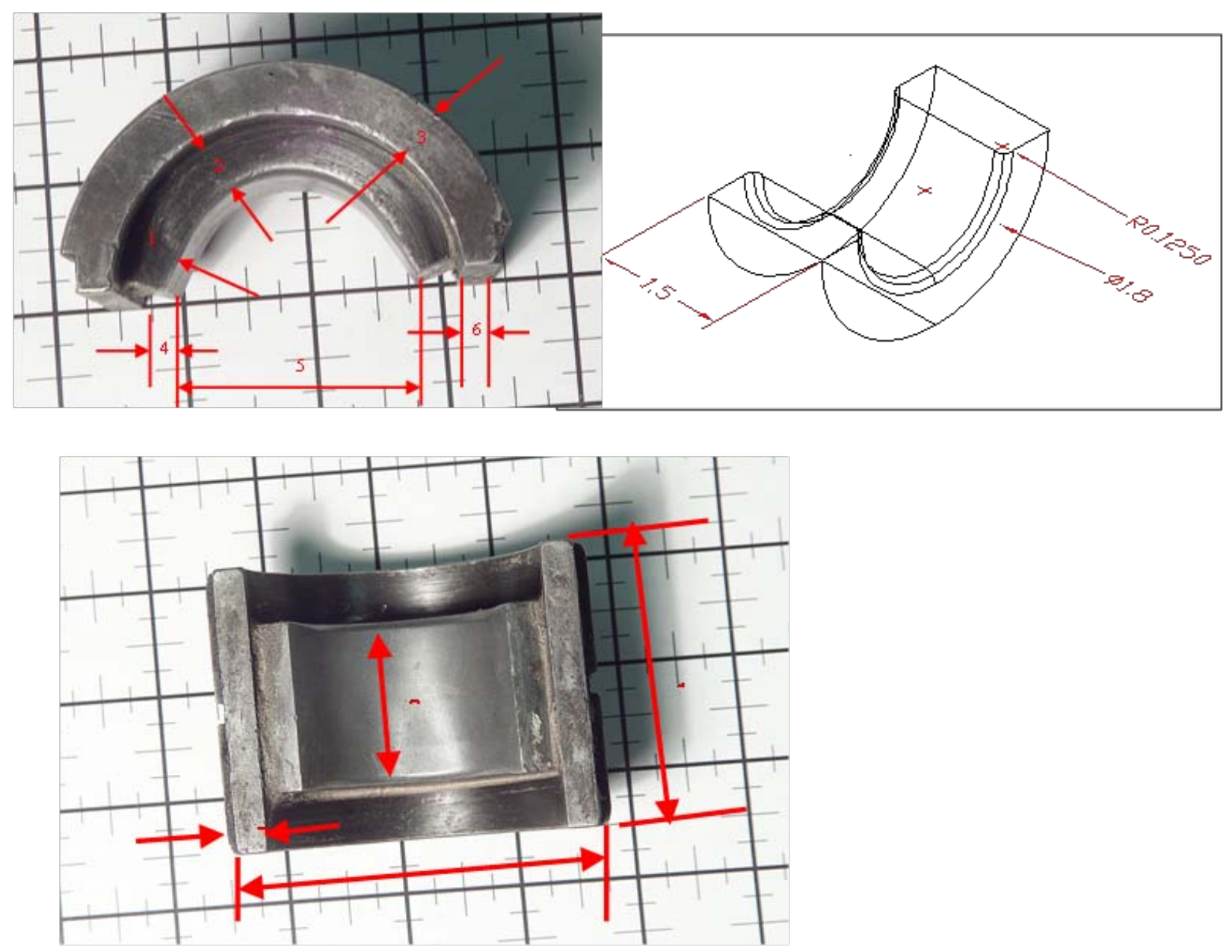

Figure 6-1 Dimensions of 6012CD Round Die Half Used for Single-stage Fittings 
A schematic diagram of a SSC and the associated materials are shown in Figure 6-2, where the splice connector can be divided into two major sections, namely, a conductor section that contains the aluminum conductor, and a core-grip section, as indicated in Figure 6-2. The detailed cross-section of half single stage splice connector is shown in Figure 6-3. The typical physical cross-section of the core-grip and conductors of Drake SCC system are shown in Figure 6-4.

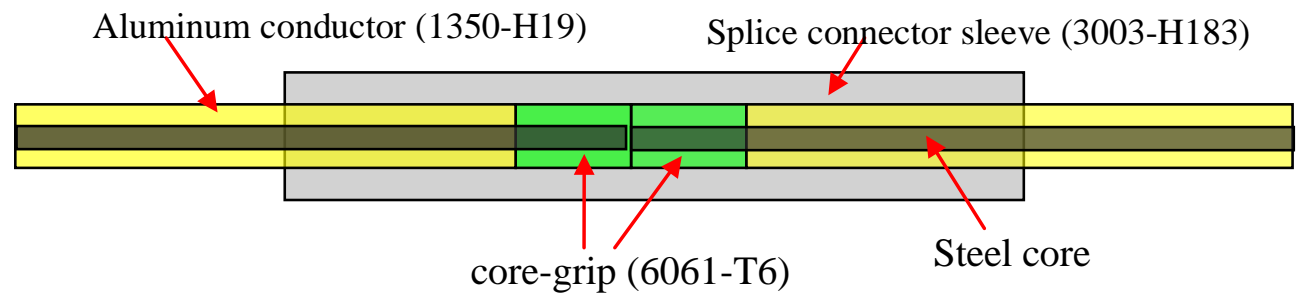

Figure 6-2 Geometry of the single stage compressive splice connector

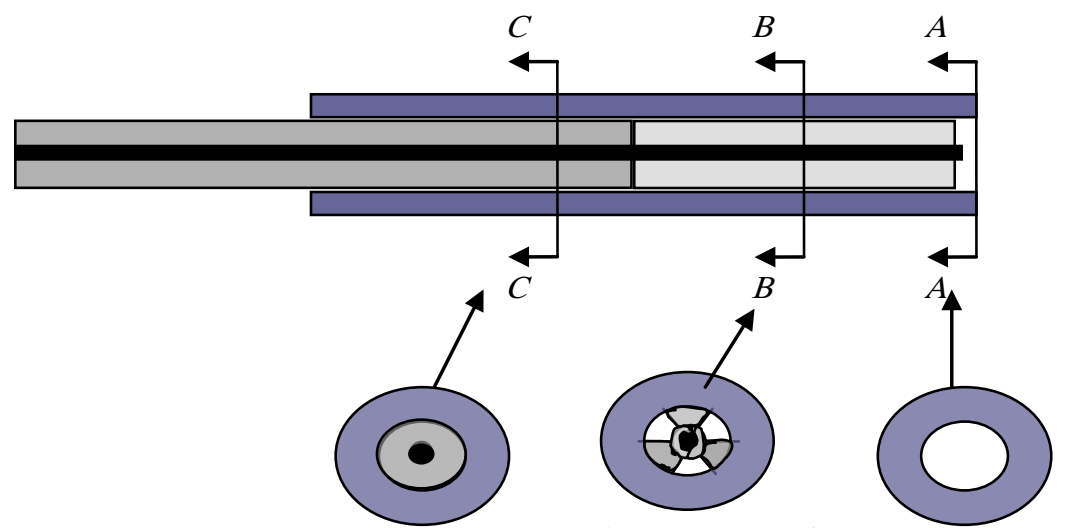

Sleeve, conductor, and steel core

Sleeve, core grip, and steel core Splice Sleeve

Figure 6-3 Detailed cross-section of a half single stage splice connector

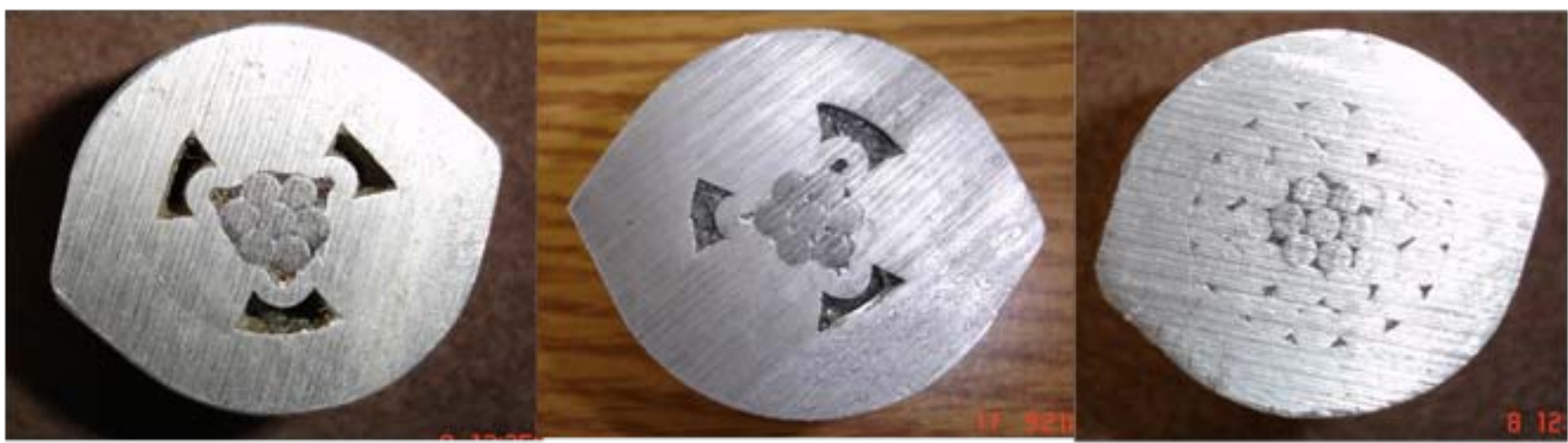

Figure 6-4 Typical cross-sections of ACSR Drake SSC system: (left, middle) core-grip section, and (right) conductor section. 


\subsubsection{Load Transfer within A SSC Fitting}

The tensile load transfer in a connector system is illustrated in Figure 6-5. In the middle section of the splice connector, where no conductor is passing through, the load transfer mechanism is simply through the connector outer sleeve as shown in the top figure in Figure 6-5. The free body diagram shown in bottom figure of Figure 6-5, illustrates the distribution of the friction resistance force induced by the compressive residual stress at the interface between sleeve and conductor and the interface between core-grip and steel core. In order to secure the conductor in the connector sleeve during a valid connector rated breaking strength test, the total shear force resistance in the crimped splice connector fitting, including the steel core and conductor section (Figure 6-5) must be greater than that of 95\% rated breaking strength of the conductor. It is expected that the friction force resistance will decrease progressively during power line operation. Therefore, the degradation of shear resistance forces provides the basis for an accurate estimate of the connector lifetime. The rated strength of a Drake conductor is equal to

$(26) \times(576.6) \times(0.93)+(7) \times(2615) \times(0.96)=31,515$ lbs $(1.40 \mathrm{E}+5 \mathrm{~N})$.

Note that no clamping is allowed at the middle section of the SSC fitting. The net cross-section of the splice sleeve at the middle of the sleeve remains the same, and equals to

$(3.14 / 4) \times\left(1.8^{2}-1.2^{2}\right)=1.414 \mathrm{in}^{2}$.

Thus, the maximum tensile load that can be sustained or transmitted by the sleeve (yielding strength, 27ksi) at ambient temperature is equal to

$27,000 \times 1.4137=38,169$ lbs.

This strength is more than the Drake conductor rated breaking strength of 31,515 lbs.

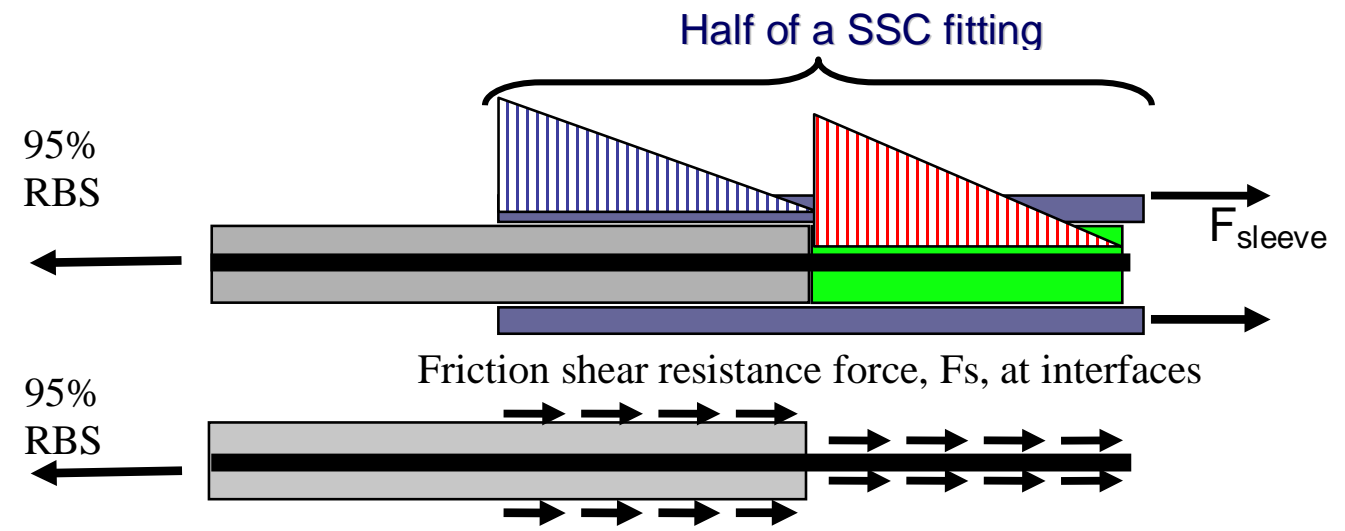

Figure 6-5 Free body diagrams of load transfer during rated breaking strength tensile test; (top) sleeve carries the full rated breaking strength at the middle section of the sleeve, the tensile stress distribution of the conductor and core steel within the SSC fitting are nonuniform as illustrated with two triangular blocks for conductor and core-grip sections, respectively; (bottom) shear force resistance at sleeve/conductor interfaces and coregrip/steel-core interface. 
The shear force resistance shown at bottom diagram of Figure 6-5 can be attributed to two interface sections: (1) interface between the sleeve and the outer layer of the conductor wires bundle and (2) interface between the core grip and the steel core wires bundle as shown in Figure 6-5. . To prevent the pull-out failure of a SSC fitting, the sum of the interfacial friction shear forces within the fitting (Figure 6-5) needs to be greater than the tensile load experienced by the conductor. It is expected that the friction force resistance will decrease progressively during operation due to damage induced by creep deformation and thermal cycling. Therefore, understanding the mechanism that induces the degradation of shear resistance forces provides the basis for an accurate estimate of the connector lifetime. PATRAN mesh generator and ABAQUS Code were used in the finite element analyses. Due to large number of contact surfaces involved during the connector crimping process, the FEM meshes were modified and optimized several times during the analyses in order to reach a better convergent solution at a reasonable computer cpu running time frame.

\subsection{FEM Residual Stress Analysis for Core-grip Section}

Due to allowing the free lateral extrusion to occur during crimping SCC fitting, a 2-D FEM approach was utilized for investigating the deformation mechanism without losing generality. 2D plane-stress constraint was used to simulate core-grip section's crimping event. The incremental loading scheme was used in nonlinear FEM analyses to simulate the crimping process until the prescribed die closing geometry was reached. Totals of 5108 4-node quad elements were used in the final simulation analysis. The FEM model for core-grip section contains four subsections, namely, die-set, connector sleeve, core-grip, and the steel core wires. In order to simulate the possible positions of the core-grip within the aluminum sleeve, three configurations were designed for FEM analyses. The detailed geometries for each scenario are shown in the top row of Figure 6-7 and the FEM simulated deformed core-grip sections are also shown at bottom of Figure 6-7. In Figure 6-7, the core-grip position shown in the first column to the left note that its three spikes are symmetric to the y-axis, identified as 3P position; and the middle column the core-grip has two edges of its spikes perpendicular to the $\mathrm{x}$-axis, identified as Vert position; and the column to the right is the modified position to the Vert with a $15^{\circ}$ rotation of the core-grip to Vert position, identified as 15D.

\subsubsection{FEM Analysis Results for 3P, Vert, and 15D Core-grip Positions}

The FEM model for core-grip section contains three layers of gaps, which are between (1) die-set and outer sleeve, (2) outer sleeve and core-grip spike regions, and (3) core-grip ring and steel core wires bundle, as shown in Figure 6-6. The friction coefficient at these interfaces is assigned as 0.3 . The one exception is the 0.6 friction coefficient that was assigned to interface between the steel core and core-grip ring where the rough particles were attached to the inner ring of coregrip. It is interesting to note here, due to the die-set geometry, the initial contact points between the die and the outer sleeve are not near the center symmetry position. 


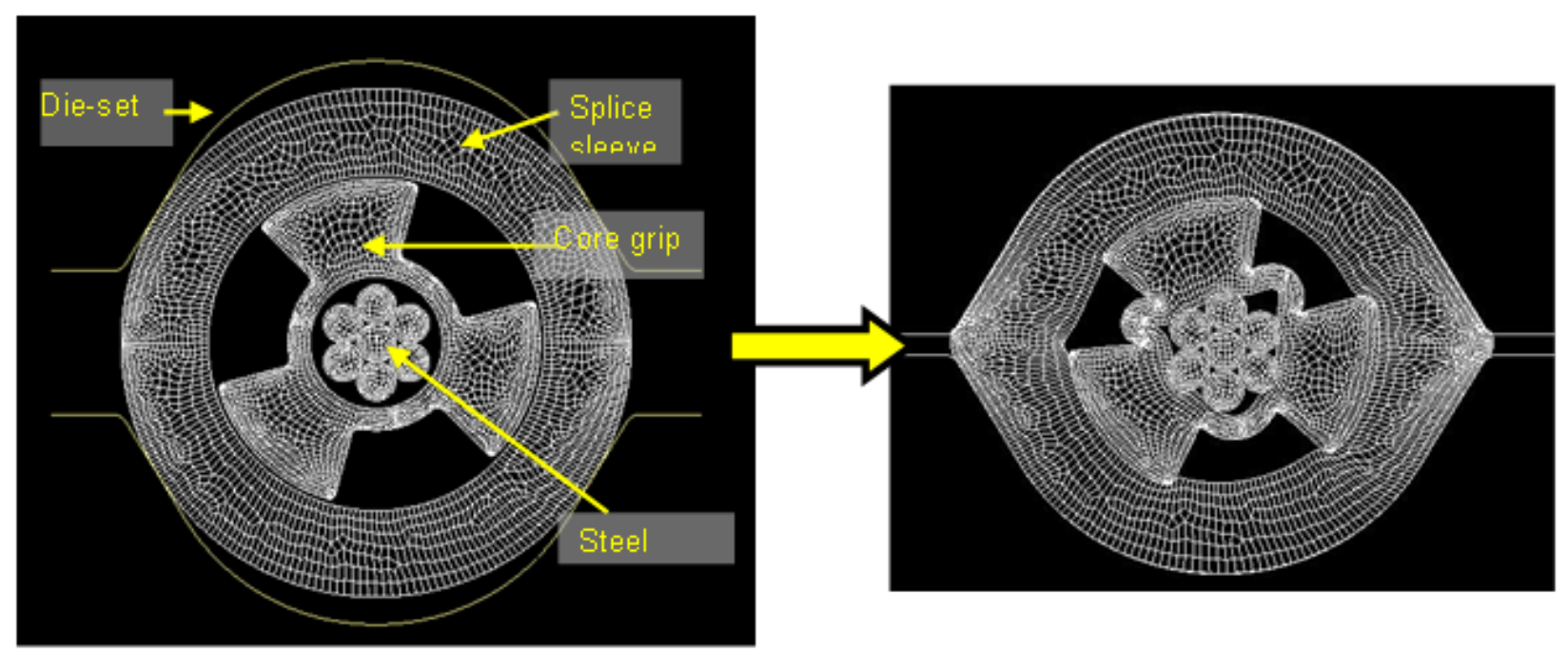

Figure 6-6 Finite element model (FEM) of an ACSR Drake SSC fitting (left) before forming, (right) after crimping.

FEM analyses indicated that the outer sleeve in the earlier stage of the crimping was deformed into an elliptical shape with the long axis aligned with the y-axis (vertical position). The long axis portion of the sleeve was not experiencing compressive stress till the later stage of crimping, where the die-set is starting to close in and contact to the long-axis portion of the deformed sleeve. Significant plastic deformations were experienced both for outer sleeve and core-grip regions. Especially for the core-grip ring region near the steel core, the ring was deformed into a triangular shape; and the "Self-contact" phenomena was also observed, which was due to an extremely large deformation of the contact surface at core-grip region which resulted in it folding onto itself as indicated in Figure 6-7. The estimated compression load required to deform 3P, Vert, and 15D positions with a 1.5 inch width of 6012CD die-set are 54.6 tons, 57.5 tons, and 56.0 tons, respectively.

The normal contact pressures distribution at the interface between core-grip and steel core wires for 15D core-grip position are illustrated in Table 6-1. Contact condition "ST" stands for contact point closed and sticking, and "SL" stands for contact point closed and slipping. The total accumulated normal compressive stress (per unit cross section) that confine the core steel are $1.13 \mathrm{E}+06 \mathrm{psi}, 1.24 \mathrm{E}+06 \mathrm{psi}$, and $1.25 \mathrm{E}+06 \mathrm{psi}$; and the accumulated friction resistance forces for a 4.5 inch long core-grip section are $3.25 \mathrm{E}+04 \mathrm{lb}, 3.56 \mathrm{E}+04 \mathrm{lb}$, and 3.60E+04 lb, for Vert, 15D, and $3 \mathrm{P}$ positions, respectively. It is interested to note that the $3 \mathrm{P}$ position required less crimped force to form it but provides the highest friction resistance among all three scenarios. Furthermore, the deformed ring section of the core-grip at 3P position appears to be more compact than other two core-grip positions. The estimated binding strengths from above 3 scenarios are all greater than that of the steel-core wires' strength, $7 \times 2615=1.83 \mathrm{E}+04 \mathrm{lbs}$ This indicates that the initial binding strength of the crimp core-grip section at room temperature is sufficient to constraint the steel core wires during the rated breaking strength test. 

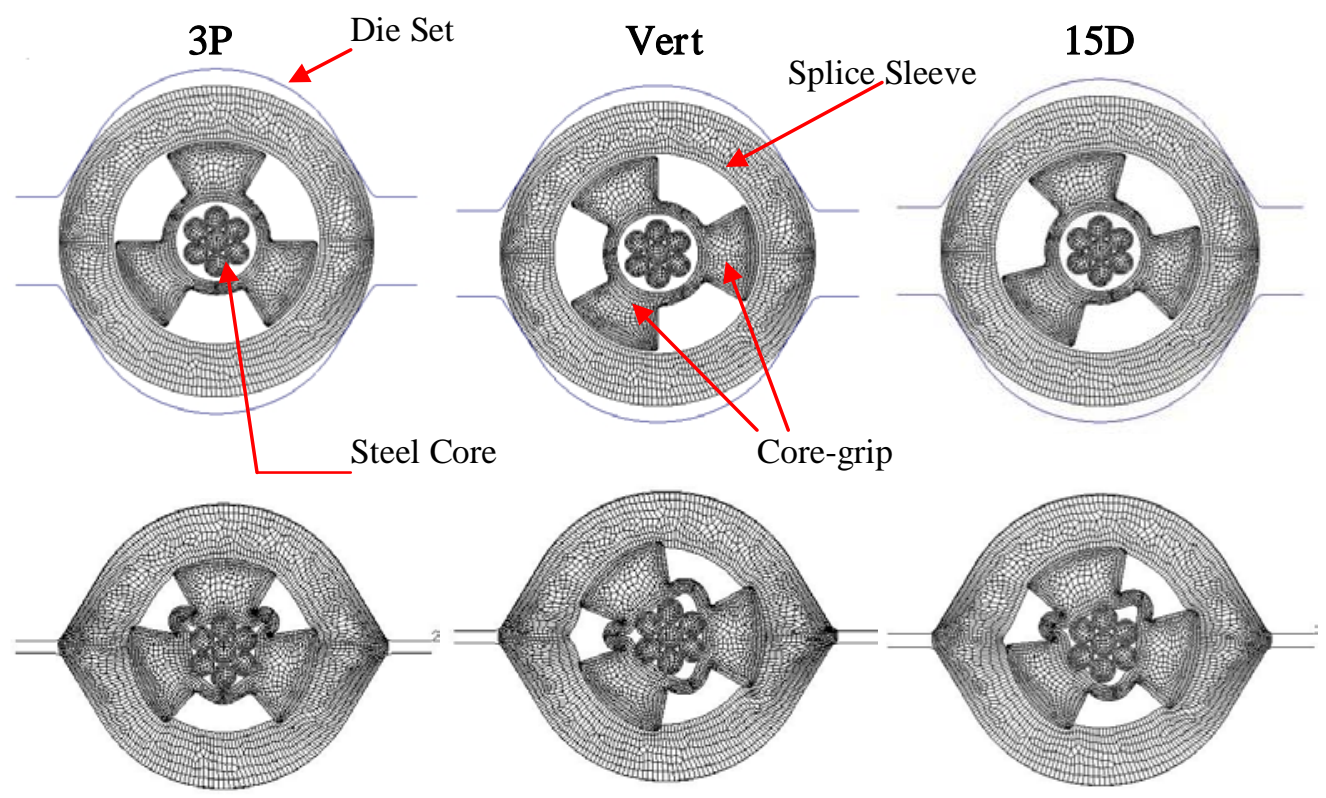

Figure 6-7 FEM models for core-grip section of Drake splice connector with 6012CD die: (top) before crimping and (bottom) crimped SCC.

Table 6-1 Normal contact pressures between core-grip and steel of 15D position

\begin{tabular}{llllll}
\hline Node & Contact Cond. & Normal Traction (psi) & Node & Contact Cond. & Normal Traction (psi) \\
\hline 21245 & ST & $1.84 \mathrm{E}+04$ & 21499 & ST & $2.59 \mathrm{E}+04$ \\
21248 & ST & $5.72 \mathrm{E}+04$ & 21500 & ST & $2.20 \mathrm{E}+04$ \\
21250 & ST & $2.88 \mathrm{E}+04$ & 21611 & SL & 8596 \\
21251 & ST & $1.44 \mathrm{E}+04$ & 21612 & SL & 5656 \\
21252 & ST & $3.33 \mathrm{E}+04$ & 21613 & SL & $5.11 \mathrm{E}+04$ \\
21254 & ST & $2.88 \mathrm{E}+04$ & 21736 & ST & $6.49 \mathrm{E}+04$ \\
21365 & SL & $2.47 \mathrm{E}+04$ & 21738 & ST & $3.07 \mathrm{E}+04$ \\
21367 & SL & $6.75 \mathrm{E}+04$ & 21739 & ST & $2.12 \mathrm{E}+04$ \\
21369 & ST & $7.66 \mathrm{E}+04$ & 21740 & ST & $1.43 \mathrm{E}+04$ \\
21490 & ST & $5.91 \mathrm{E}+04$ & 21742 & ST & $7.17 \mathrm{E}+04$ \\
21492 & ST & $1.16 \mathrm{E}+05$ & 21745 & ST & $9.60 \mathrm{E}+04$ \\
21495 & ST & $1.16 \mathrm{E}+05$ & 21747 & ST & $2.57 \mathrm{E}+04$ \\
21497 & ST & $1.05 \mathrm{E}+04$ & 21861 & ST & 7504 \\
21498 & SL & $5.31 \mathrm{E}+04$ & 21863 & ST & $9.80 \mathrm{E}+04$ \\
\hline \multirow{2}{*}{ Sum } & & 21865 & ST & 3400 \\
\hline
\end{tabular}

The effective von Mises stress fringes at the die-set closure and after the removal of the die-set for 3P and 15D positions are shown in Figure 6-8. It is noted here, due to incorporating the three layers of gaps into FEM modeling, the artificial dynamics damping coefficient was implemented into FEM analysis in order to stabilize the numerical solver and to prevent rigid body motion 
induced instability or singularity. These artificial damping coefficients will reduce to zero when the gap was eliminated due to contact closure, and it will have no impact to the final solution.
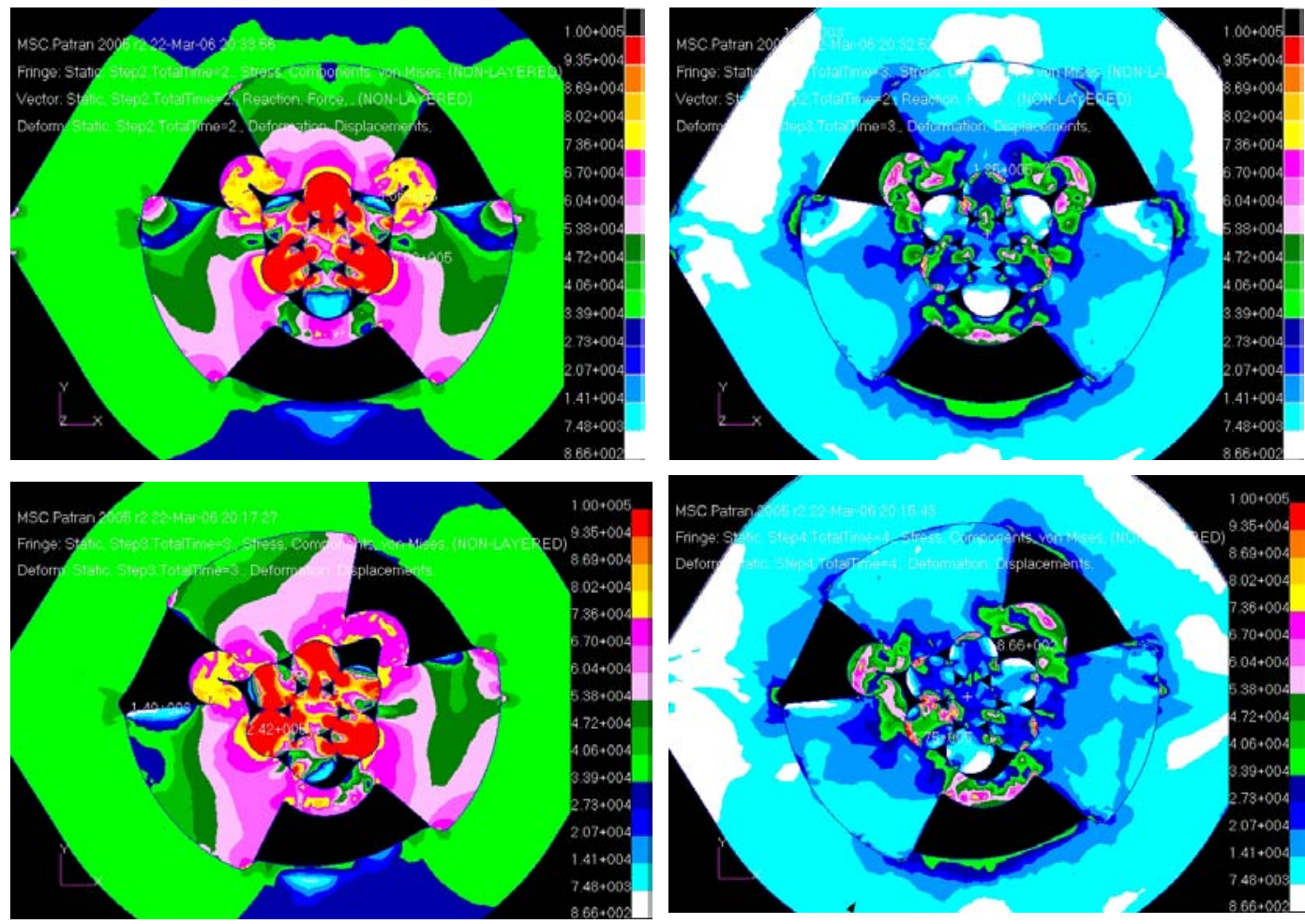

Figure 6-8 von Mises effective stress fringes of FEM analysis for 3P (top) and 15D (bottom) core-grip positions: (left) at the die-closure; (right) after the removal of the die-set, which represents the effective residual stress field remained in the core-grip section.

The effective von Mises stress fringes at the die-set closure and after the removal of the die-set for Vert position are shown in Figure 6-9. The relaxation of clamping compressive residual stresses at steel core/core-grip interface after die-removal reaches $77 \%$.
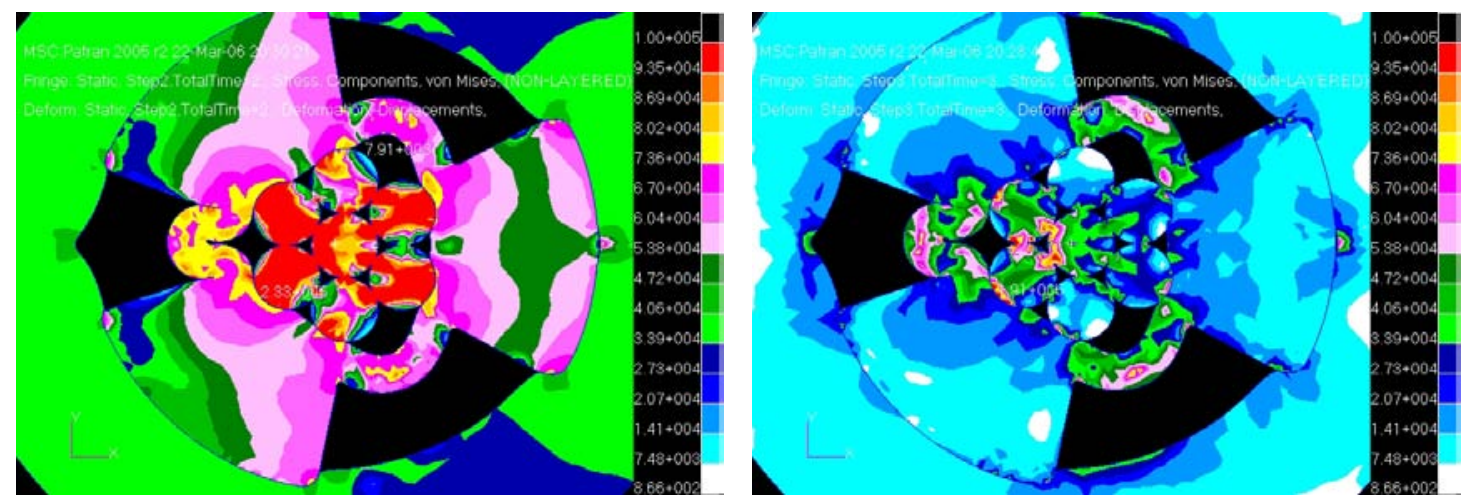

Figure 6-9 von Mises effective stress fringes of FEM analysis for Vert core-grip position: (left) at the die-closure; (right) after the removal of the die-set. 
The FEM analyses provide detailed insight of the contact deformation among the constituents within the core-grip and the effective stress states of a deformed SSC at the core-grip section. This provides the basis for quantitative estimates of the friction force induced by compressive residual stress within the core-grip region upon completion of forming the crimped splice connector.

\subsection{FEM Residual Stress Analysis for the Conductor Section}

Initially a 2-D fine mesh FEM model with plane-stress constraint was used for simulating the crimping deformation mechanism at conductor section illustrated in Figure 6-10. A total of 6,200 2-D 4-node quad elements were used in the FEM model, the total number of the projected potential contact-surface pairs are about 48 . These contact-surface pairs need to be adjusted accordingly during the crimping loading simulation. Thus, frequent intervention with the FEM analysis is necessary to update the changes of states of these potential contact pairs. The final estimates of the shear force resistance between the sleeve and the outer conductor layer appear to be fairly small. This is mainly due to 2-D conductor simulation, where the cross-spiral constraints between conductor layers can not be simulated. The cross-ply spiral formation of conductor layers will resist compactness of the conductors during crimping more efficiently than that of 2-D simulation. Furthermore, for a unit volume the 3-D spiral conductor occupies more volume than that of a 2-D conductor model, thus logically 3-D models will also require more crimping force to deform the connector, and this also results in larger residual compressive stress.

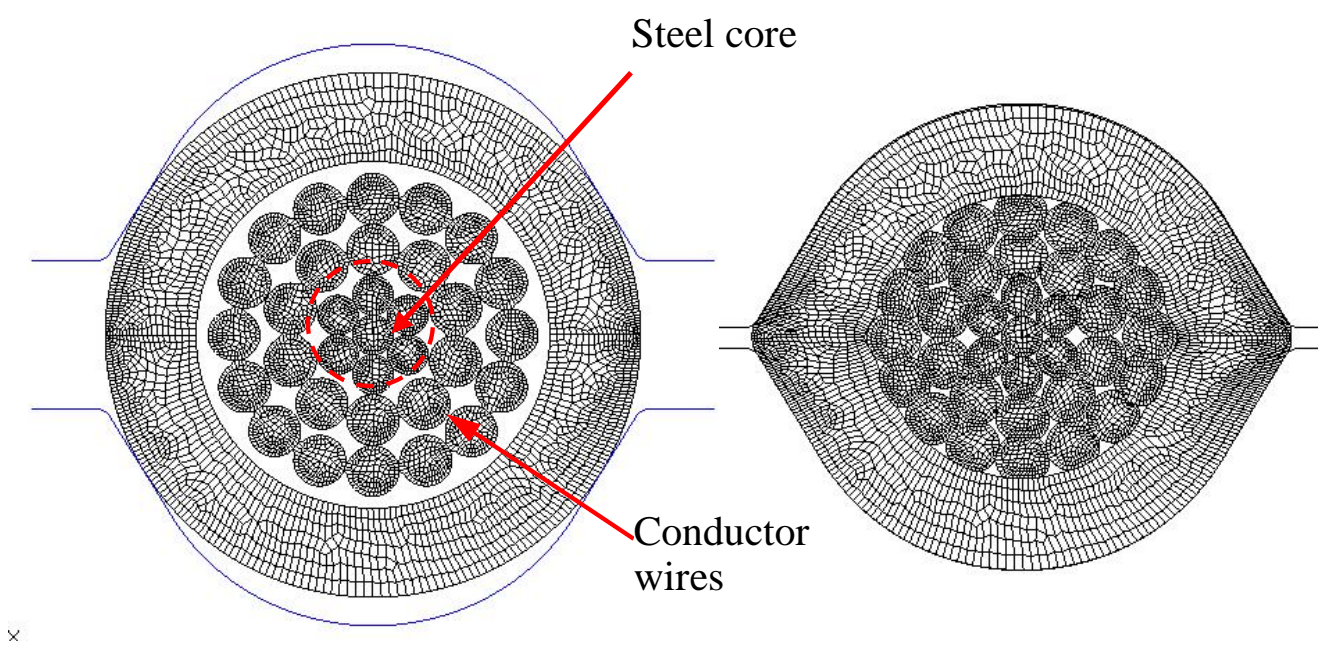

Figure 6-10 2-D FEM forming simulation for conductor section: (left) un-deformed baseline, (right) crimped SCC at die closure.

Due to ACSR conductors' 3-D spiral configuration, 3-D FEM models were used for simulating the SSC forming in the conductor section. Due to significant number of the contact surfaces existing in the FEM model, including 26 conductor wires and 7 core steel wires (the potential contact orientations are shown in), the EXPLICT ABAQUS Code was used in the FEM analysis; where the dynamics analysis scheme was utilized with "General Contact" approach. Several fine meshed FEM models were used in the investigation, but it took a long time to reach a convergent solution. 


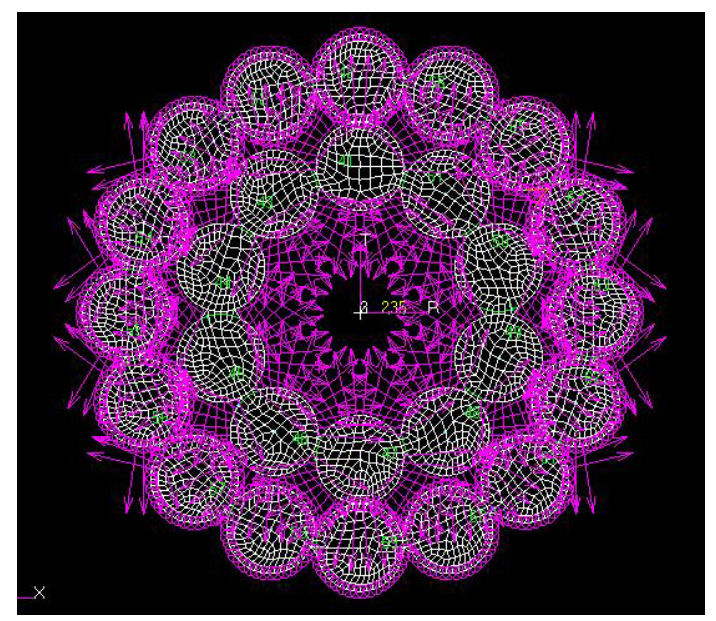

Figure 6-11 Normal directions of the potential contact surfaces for aluminum conductor layers.

In order to solve the FEM model with a reasonable time frame, a relative coarse mesh, with 18,880 solid 3-D 8-node brick elements, was designed for simulation and will not lose the generality. The un-deformed 3-D FEM model is designed to cover a single die-set length of 1.5 inches with the sufficient length of the sleeve and the Drake conductor for simulating SCC crimping event, as shown in Figure 6-12. The simulated deformed conductor section at the middle section of the FEM conductor model is shown in Figure 6-12.

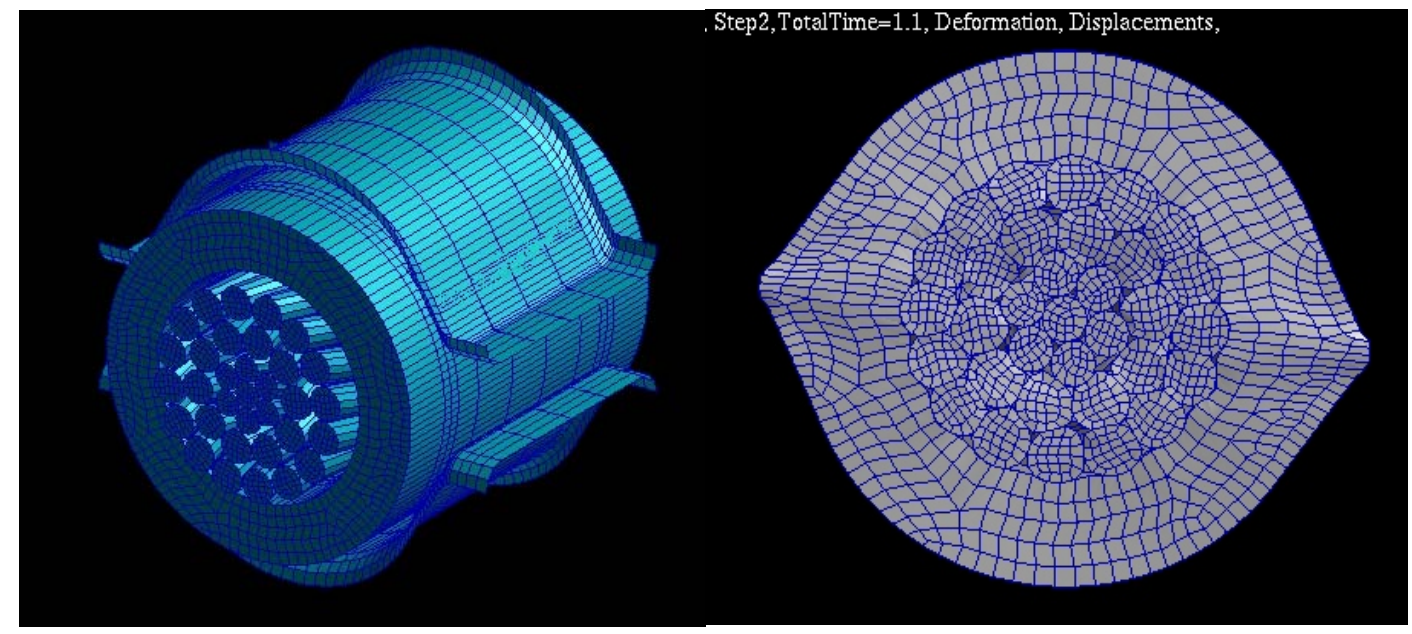

Figure 6-12 (Left) Un-deformed baseline SSC FEM model at conductor section, (right) crimped conductor section at the middle section of FEM model after the die-set removal.

Figure 6-13 shows deformed splice connector at die-set closure. The side view of Figure 6-13 indicates an uneven lateral extrusion phenomenon at two ends of die-set, where the top portion of sleeve has less lateral extrusion compared to that of middle portion of sleeve. This is the result of the contact loading sequences of die-set during the crimping event induced by the geometry of a 6012CD die-set. Therefore, the final deformation of a crimped single stage connector fitting is normally slightly curved up or down along the conductor's axial orientation. 


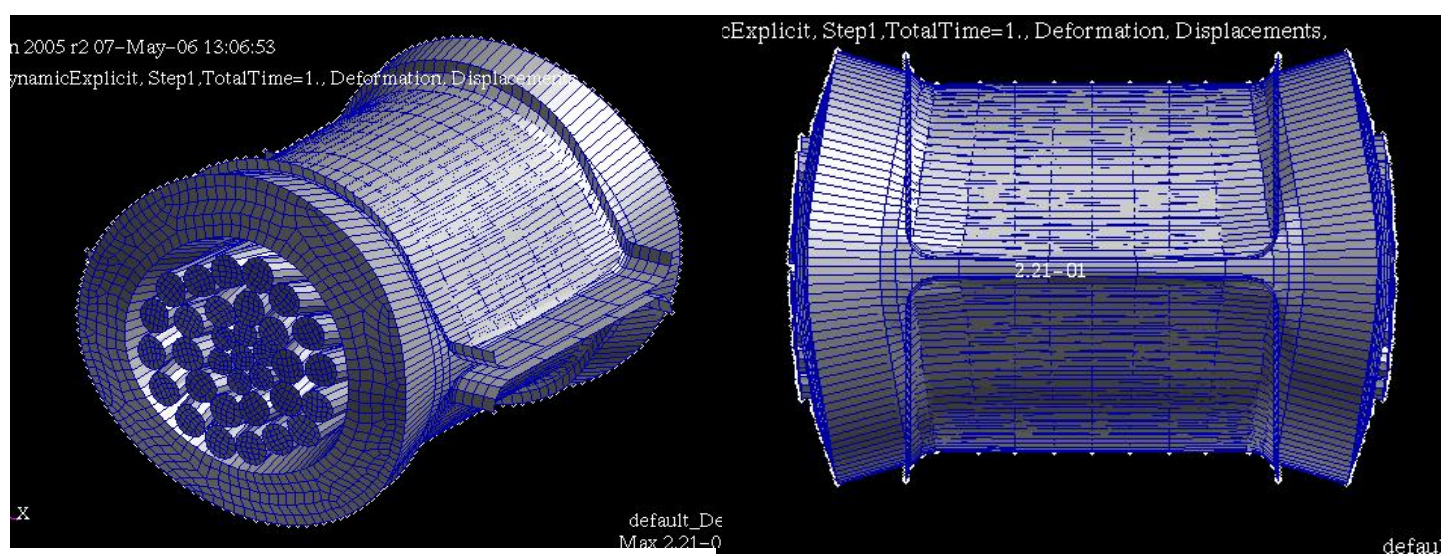

Figure 6-13 FEM simulated deformed configuration for SSC fitting at the conductor section upon die-closure: (left) iso-view, (right) side-view.

Figure 6-14 show the von Mises stress fringes of a crimped splice connector at the conductor section before and after the die-set removal, respectively. The compressive residual stresses between interface of the splice sleeve and the outer layers of the conductor wires provide the binding strength to secure the Drake conductor cable during the rated breaking strength test or during the conductor service lifetime. The relaxation of clamping compressive residual stresses at sleeve/conductor interface after die-removal reaches $88 \%$.

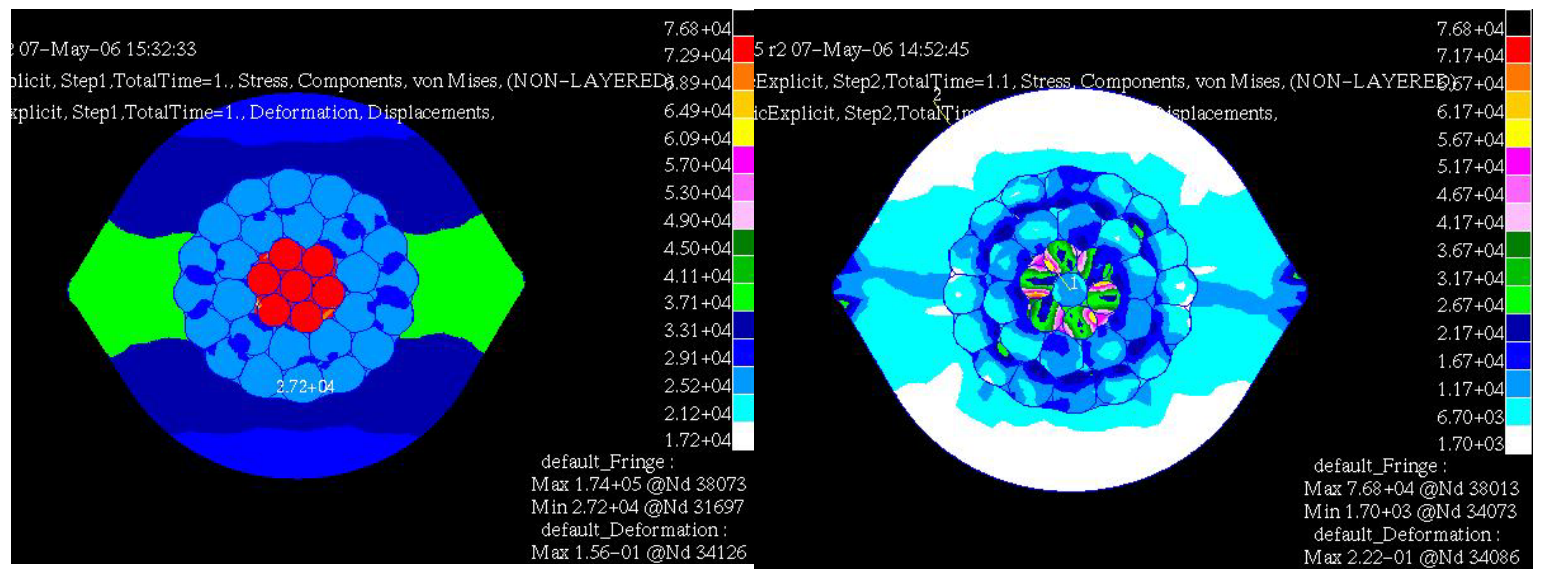

Figure 6-14 von Mises stress contours at middle layer of a crimped SSC conductor section: (left) at die-set closure, (right) after die-set removal.

The estimated hydraulic crimping force required to form the splice connector using a 6012CD die-set in the conductor section is about 54 tons per die. The effective friction resistance forces induced from the compressive residual stress within the crimped connector at a conductor section with effective length of 3.5 inch (w/ consideration of the taper section of the sleeve) is about $8,046 \mathrm{lbs}$. Here the friction coefficient is assigned as 0.3 between aluminum conductor and the aluminum sleeve. As for a friction coefficient of 0.6 (if no grease at the interface) the friction resistance at interface induced by the compressive residual stress within the conductor region will be 16,092 lbs. For a rated breaking strength tensile test according to Southwire Conductor Handbook, the Drake conductor will contribute about $26 * 576.6 * 0.93=13,942 \mathrm{lbs}$. For a friction 
coefficient of 0.6, the friction resistance force is $16,092 \mathrm{lbs}$. This is sufficient to confine the conductor wires in the conductor section during rated breaking strength tensile test. As for a 0.3 friction coefficient, the friction resistance force alone at the interface in the conductor section will not be sufficient during the rated breaking strength test to confine the aluminum conductors. However, due to 3-D spiral configuration of the conductor wires, the indented spiral groove on the inner surface of the splice connector will kink or bend the outer layer's conductor wires during axial tension movement (conductor tension test is inclined to the spiral groove at the conductor pitch angle) This in turn will provide additional resistance during the rated breaking strength test. Nevertheless, effectiveness of this type's of resistance is also expected to be decreased progressively during thermal cycles due to gap increased between the sleeve and conductor, especially for a high temperature operation. From a crimped splice point, the grease or lubricant at interface will be removed and the friction coefficient of 0.6 is considered to be appropriate number for calculating the friction resistance force at conductor section at room temperature.

\subsection{In-Situ Non-Destructive Evaluation of the Residual Stresses within A Crimped Splice Connector}

In order to benchmark the residual stress profile within the clamped splice connector, a nondestructive evaluation using neutron scattering technique for determining the residual stress profile within the clamped connector fitting was carried out. This residual stress mapping experiment was sponsored by the ORNL HTML User Program and was carried out at the ORNL High Flux Isotope Reactor (HFIR) Facility in January 2006. The NRSF2 highest flux research instrument and support at HFIR facility provides unique capability and a state-of-the-art knowledge base for materials science research on measuring strains and strain gradients in materials. A $2 \theta$ goniometer with large $\mathrm{XYZ} / \Omega$ mapping stages and a 7 detector array were provided at NRSF2. NRSF2 set-up has multiple wavelengths for different materials, and is able to conduct measurements of strains for multiple hkl's lattice plans. A $25 \mathrm{kN}$ linear electric actuator load frame allows in-situ neutron measurement under tensile or/and compressive load. Here, the author would like to acknowledge the excellent support received from Drs. Ke An and Cam Hubbard to successfully carry out the residual stress evaluation experiment.

The experiment was completed after three days of neutron beam time. The joint compound /inhibitor contained in the crimped splice has strong effects in slowing down the neutron and reducing the intensity of diffracted neutrons. Thus, a longer beam time is required for each targeted position, in order to have better resolution of residual strain mapping within the crimped splice connector fitting.

\subsubsection{Basic Concept of Measuring Residual Strain by Neutron Diffraction Strain Mapping}

In a neutron scattering technique, the lattice spacing, $d$, is determined according to Bragg's law as illustrated in the following formula and in Figure 6-15,

$$
d=\frac{\lambda}{2 \sin \theta_{p k}}
$$




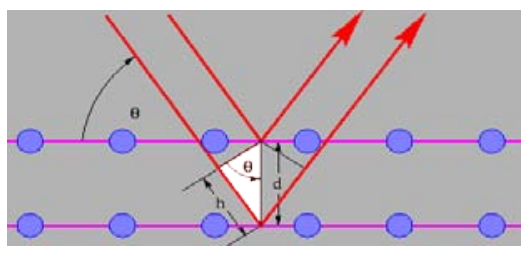

Figure 6-15 Neutron diffraction in a lattice structure.

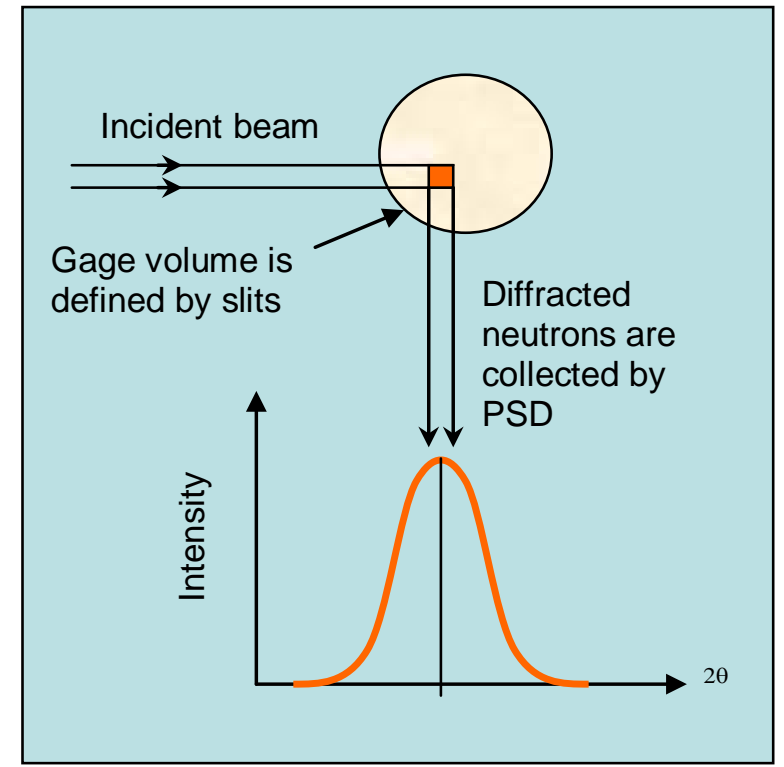

Figure 6-16 Schematic diagram of neutron diffracted by the target material and using PSD detector to determine the peak angle, $\theta_{p k}$.

and, lattice strain is given by

$$
\varepsilon=\frac{d-d_{0}}{d_{0}}
$$

where, $d_{0}$ is the strain free d-spacing

Upon determination of the residual strain in X, Y, and Z-coordinates, the residual principal stress can then be calculated according to Hooke's law with three strain components as described below:

$$
\begin{aligned}
& \sigma_{x}=\frac{E}{(1+v)(1-2 v)}\left[(1-v) \varepsilon_{x}+v\left(\varepsilon_{y}+\varepsilon_{z}\right)\right] \\
& \sigma_{y}=\frac{E}{(1+v)(1-2 v)}\left[(1-v) \varepsilon_{y}+v\left(\varepsilon_{x}+\varepsilon_{z}\right)\right] \\
& \sigma_{z}=\frac{E}{(1+v)(1-2 v)}\left[(1-v) \varepsilon_{z}+v\left(\varepsilon_{x}+\varepsilon_{y}\right)\right]
\end{aligned}
$$




\subsubsection{Experimental Set-up and the Test Results}

The detailed illustration of the crimped sample mounted at NRSF2 is shown in Fig. 41. The tested samples, including baselines and the crimped connector fitting, the lattice plans, and neutron beam orientation and position of the test samples are outlined below,

- Sample:

- A half-cut clamped fitting sample with joint compound

- Unclamped sleeve and core-grip

- 1350-H19 Aluminum conductor wire and core steel wire

- hkl's measured: $\mathrm{Al}$ [311] at $101.5^{\circ}, \mathrm{Fe}$ [211] at $107.7^{\circ}$

- Wavelength: $0.189 \mathrm{~nm}$ from monochromator Si400

- Slits: 1x10x1 mm

- Two axial positions of the crimped connector were evaluated, at core grip and below the core grip at conductor section as indicated with red marker on the mounted connector shown in Figure 6-17.

- Residual strains in radial (normal) direction were measured in $120^{\circ}\left(130^{\circ}\right)$ to the long axis direction of the oval shape crimped splice connector, as indicated with red line in Figure 6-18.

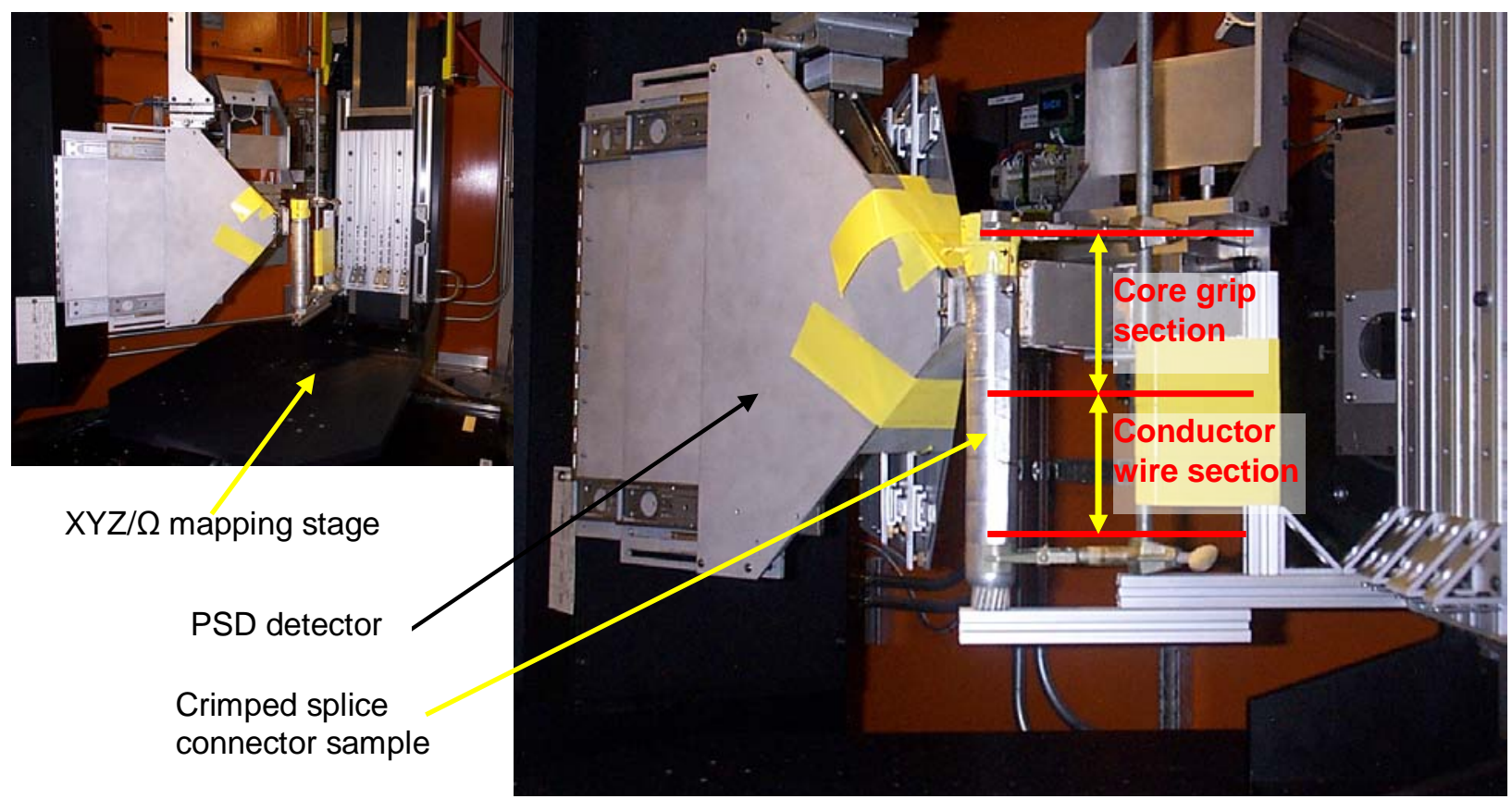

Figure 6-17 Experimental set-up at NRSF2 facility for residual strain measurement of a crimped connector

The details of the test results for the radial residual strain at the core grip and aluminum conductor sections are shown in Figure 6-19 and Figure 6-20, respectively. The radial residual strain profile is measured at $120^{\circ}$ to the long axis direction of the crimped connector fitting, which is in line with the top spike of the core grip. The reason of choosing this angle is due to the higher stress intensity being observed at the top and bottom spikes of the core grip, as learned from the nonlinear FEM analysis results in section 6.3. In Figure 6-19 and Figure 6-20, the horizontal line marker represents the baseline data for undeformed aluminum wires and the steel- 

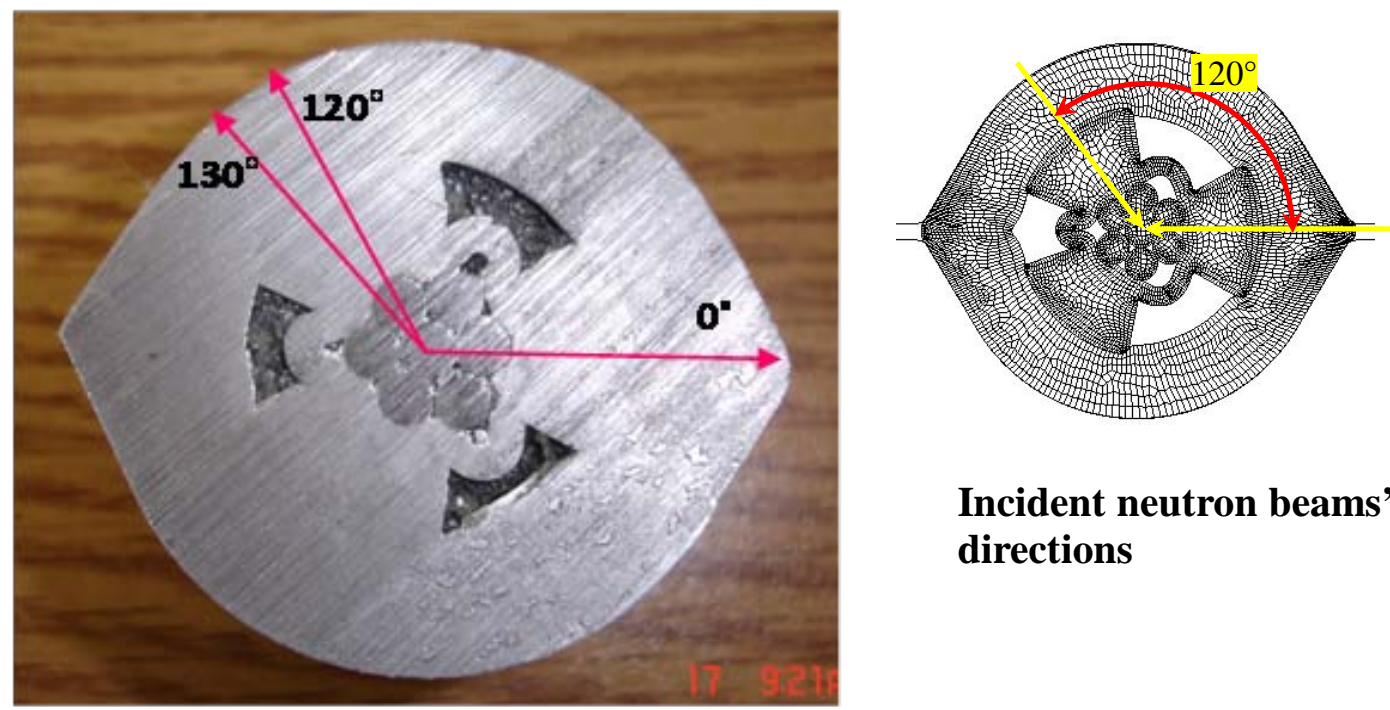

\section{Incident neutron beams'} directions

Figure 6-18 The direction of the radial residual strain profile, as indicated with red line, is about $120^{\circ}$ to the long axis of the deformed crimped sample.

core wires. The finding of this experiment is summarized below.

(1) Radial residual strain at the core grip section, shown in Figure 6-19:

- Strains in both outer sleeve and the core grip regions appear to be compressive.

- The compressive strains in the core grip are much higher than those in the outer sleeve.

- It is interesting to note that the lattice spacing on the surface of the outer sleeve remains the same before and after the crimping. Thus, no mechanical property is expected to be changed on the surface of the aluminum sleeve. This data reached the same conclusion as that from surface data obtained from ABI testing.

- From the curvature of the baseline data, it can be implied that there is residual strain in the unclamped sleeve. This may be due to forming processes used to produce the baseline aluminum sleeve.

(2) Radial residual strain measured at the aluminum conductor section, shown in Figure 6-20:

- Compressive strain also exits at the sleeve of conductor section. When approaching the conductor interface, it seems to have slightly larger strain intensity compared to that of the sleeve at the core grip section.

- Mixed tensile and compressive strains exist in the region of the conductor wires. And their strain intensities are considerably smaller than that of core grip section. This may imply that the core grip region can provide much higher confinement or constraint to the steel core compared to that of the aluminum conductor region. This phenomenon further support "The contribution to the steel core confinement by the aluminum conductor region is much less than that of the core grip region in a single-stage connector fitting. 


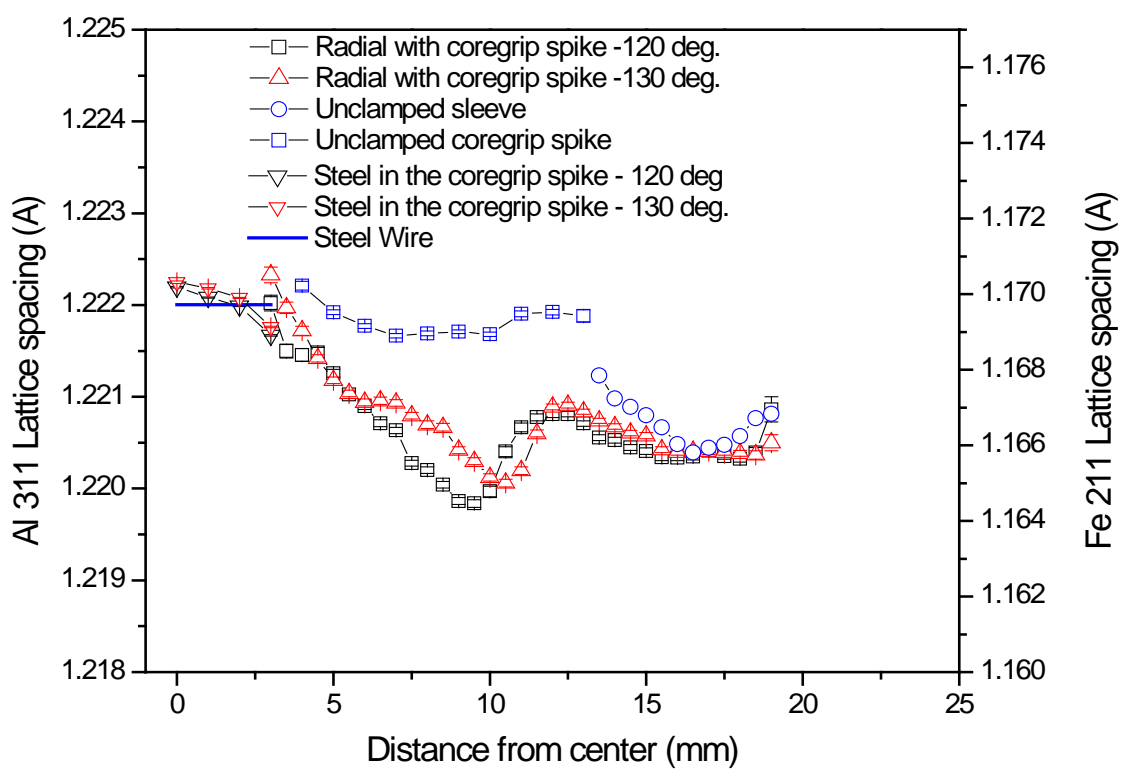

Figure 6-19 Radial lattice spacing profile at core grip section.

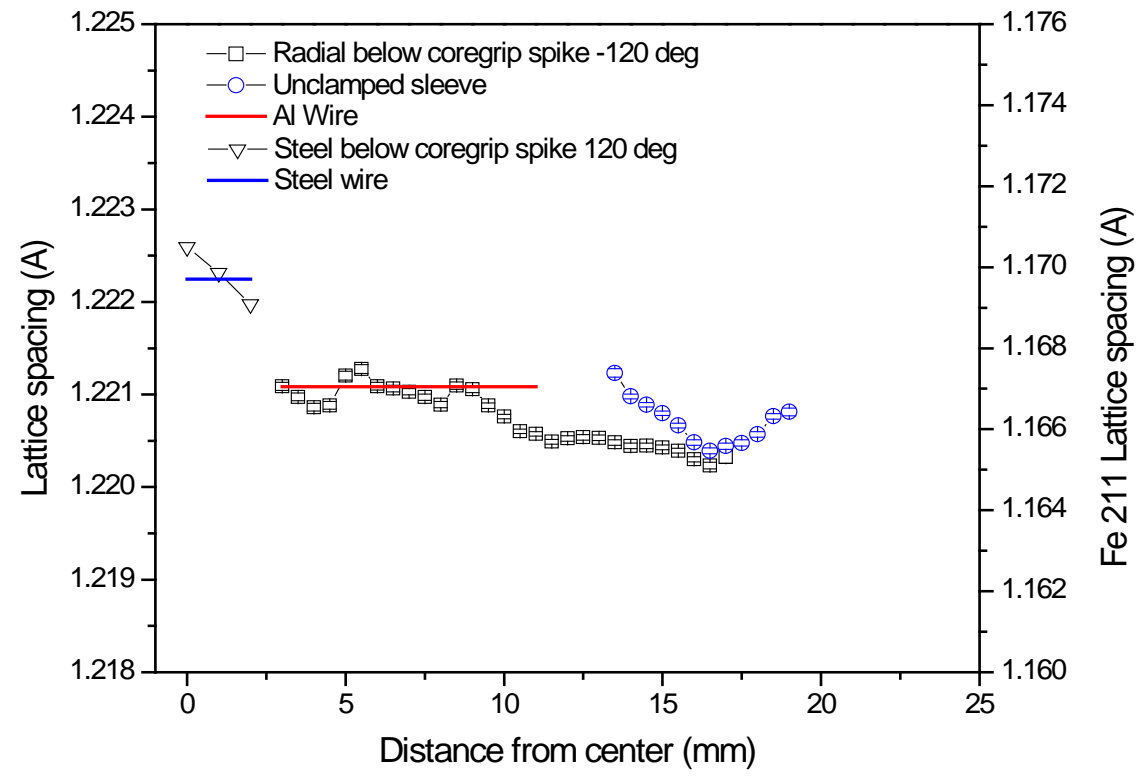

Figure 6-20 Radial lattice spacing profile at the aluminum conductor section 


\subsubsection{Comparison of Residual Stress Data from FEM Analyses and HFIR Neutron Scattering Experiments}

The irradiated connector samples used in January 2006 HFIR Experiments were sectioned to examine the detailed deformation of the splice connector, which are shown in Figure 6-21, for core-grip section and conductor section, respectively. The simulated deformation from FEM analyses closely resembles to that revealed from postmortem examinations.

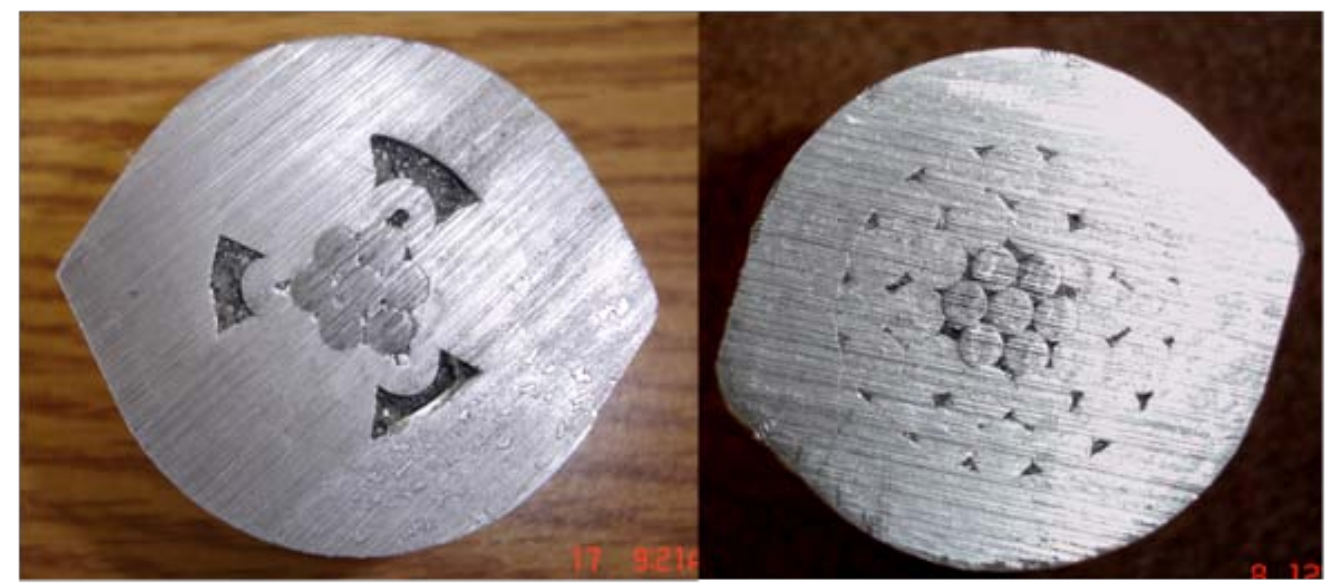

Figure 6-21 Cross-sections of the irradiated SSC samples: (left) core-grip section, (right) conductor section.

The radial residual strain profile at core-grip section is measured at $120^{\circ}$ to the long axis direction of the crimped connector fitting, which is in line with the top spike of the core grip, as shown in Figure 6-18. The deformation displacement of the core-grip section in the radial direction along the neutron incident beam at $120^{\circ}$ is illustrated in the Figure 6-22.

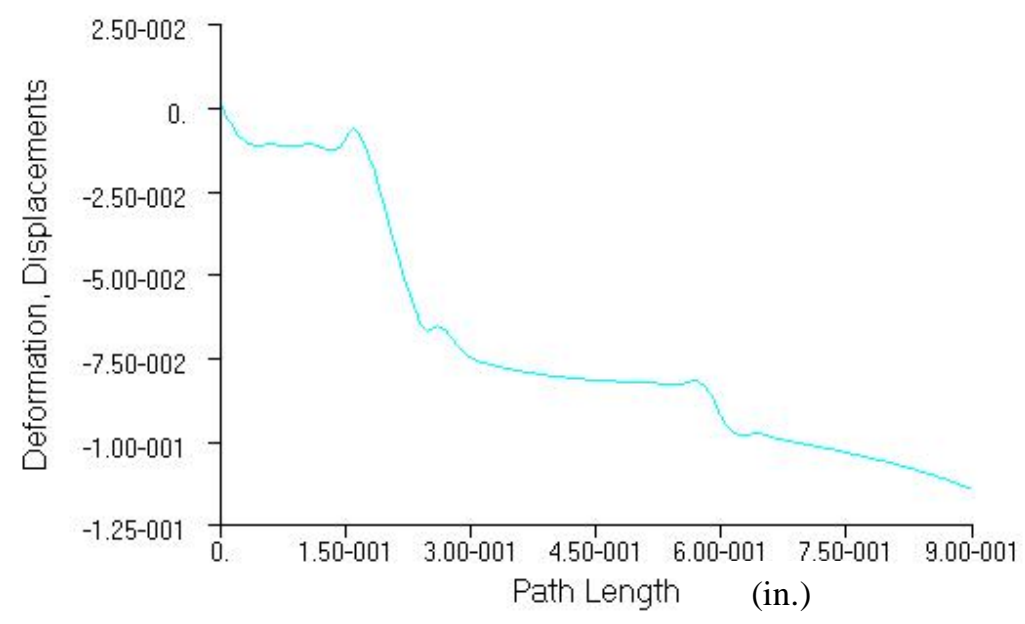

Figure 6-22 Radial displacement at core-grip section along $120^{\circ}$, path from center steel core to the outer aluminum 
The residual strain and residual stress at core-grip section along $120^{\circ}$ and $130^{\circ}$ neutron incident beam directions, evaluated from HFIR neutron scattering experiments, are shown in Figure 6-23 and Figure 6-24, respectively.

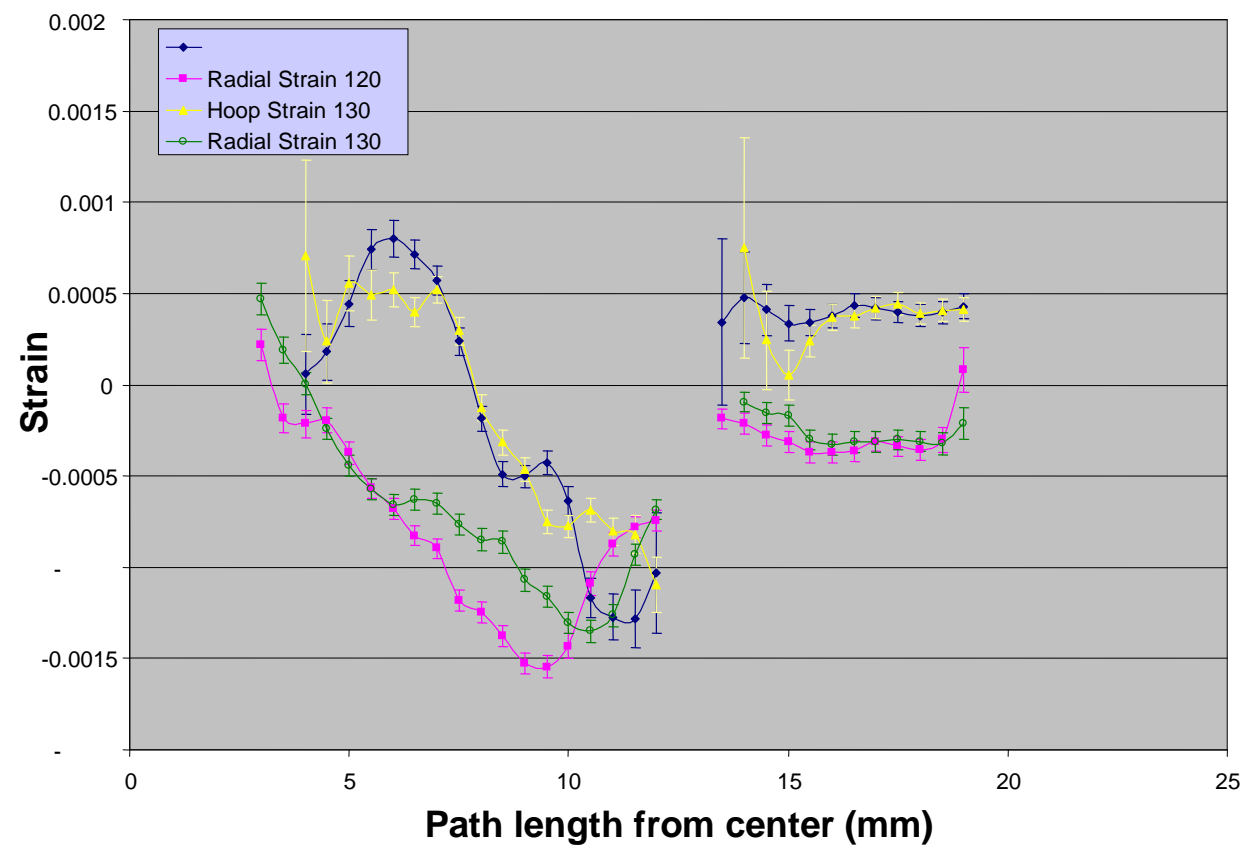

Figure 6-23 Residual elastic strain in core-grip section at $120^{\circ}$ and $130^{\circ}$, the X-axis is the deformed coordinate.

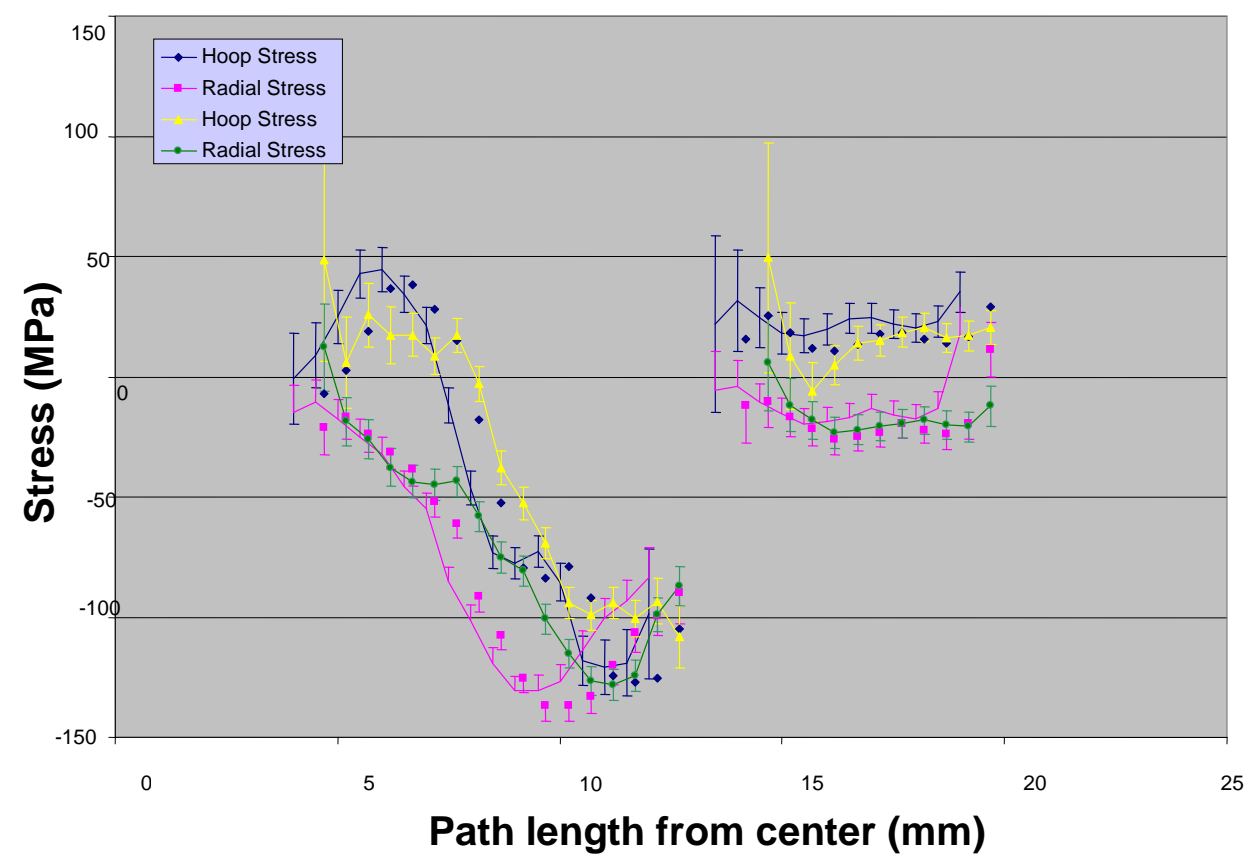

Figure 6-24 Residual stress in core-grip section at $120^{\circ}$ and $130^{\circ}$, the $X$-axis is the deformed coordinate. 
The corresponding radial residual strain and radial residual stress along $120^{\circ}$ and $130^{\circ}$, evaluated from FEM analyses, are shown in Figure 6-25 and Figure 6-26, respectively. It is noted here that the X-axis in the Figure 6-25 and Figure 6-26 is the original undeformed coordinate and the units are in inches for distance and psi for stress. For instance, in the undeformed coordinate, the radial distance of 0.4 inches is equal to 3.25 inches in a deformed coordinate, and 0.9 inch radial distance of undeformed coordinate is equal to 0.785 inch in a deformed coordinate. By comparing to the plots within the core-grip region, the maximum elastic radial elastic strain of FEM analysis along $120^{\circ}$ and $130^{\circ}$ are between 0.0012 and 0.0016 , which are similar to that of HFIR experiment, shown in Figure 6-24. While as for the maximum radial residual stress in the core-grip region along $130^{\circ}$ direction, both approaches reach the similar result about 20,000 psi.

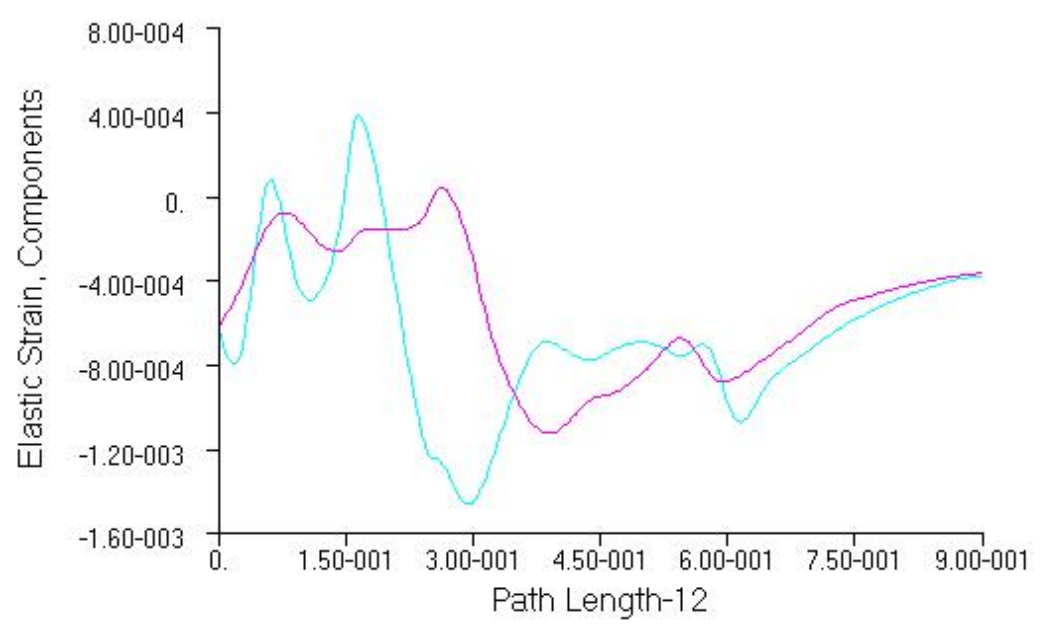

Figure 6-25 Residual elastic strain in radial direction along the $120^{\circ}$ (blue line) and $130^{\circ}$ (purple line) neutron beam directions. $X$-axis is the original undeformed mesh coordinate

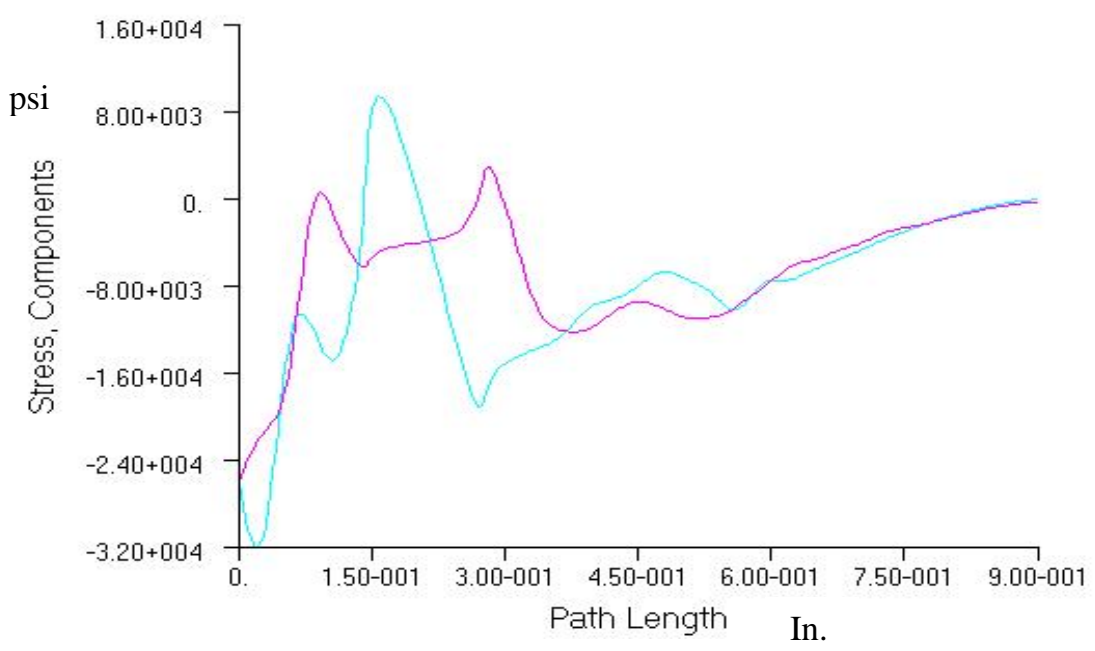

Figure 6-26 Residual stress in radial direction along the $120^{\circ}$ (blue line) and $130^{\circ}$ (purple line) neutron beam directions. X-axis is the original undeformed mesh coordinate 


\subsection{Conclusion}

Residual strains determined by neutron diffraction revealed high compressive radial deformation along the $120^{\circ}$ and $130^{\circ}$ directions at the core-grip region, shown in Figure 6-18, but relatively small strains at the aluminum outer sleeve region. These results are in agreement with the general trends predicted by FEM stress analysis. The radial compressive stress distribution is the key parameter to assess the crimping pressure strength that is responsible for securing the conductor within a crimped splice connector. Due to the complex geometry of the SSC fitting component and the large plastic deformation that occurs during the crimping process, the FEM analysis results provide better details of the stress distribution than the average data obtained from the neutron diffraction measurements. In general, along the radial direction the FEM analysis results are in good agreement with the experimental measurements, as shown in Figure 6-27. The bounds obtained from the neutron diffraction measurements also validate the FEM analysis results.

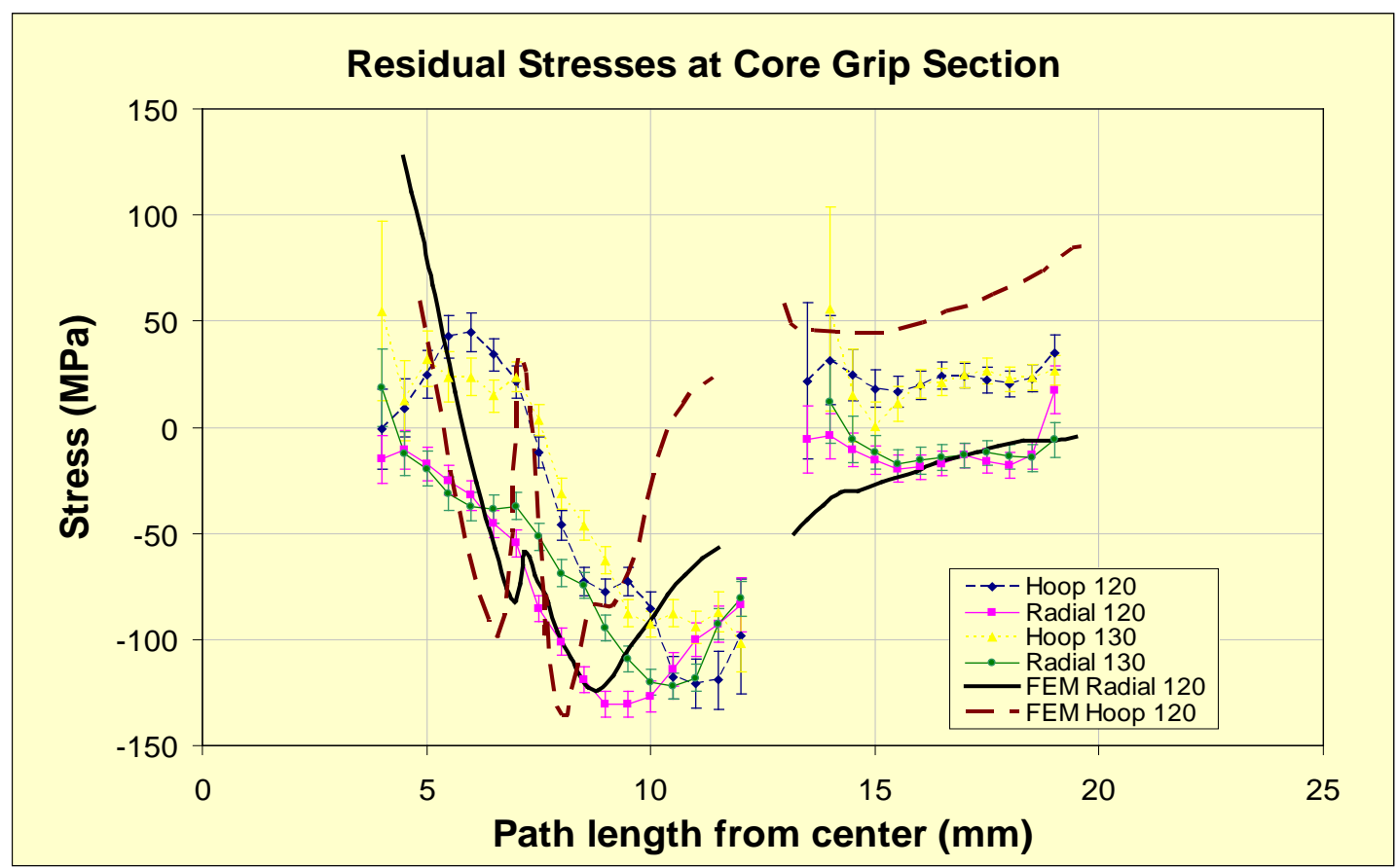

Figure 6-27 Comparison of residual stress profiles at core-grip section.

The residual stress analysis indicates that at the completion of the crimped SSC, or at the initial stage of the conductor life under normal operation, the crimped core-grip section has sufficient binding force to retain the steel core. For a 0.6 fraction coefficient of the AL-AL interface, the compressive residual stress in the conductor section also provides sufficient binding strength to confine the aluminum conductor wires during the rated breaking strength test. The additional binding strength from the conductor section can be attributed to the 3-D spiral conductor wires, where the inclined conductor wires are at pitch angle to the axial direction (or tension direction). Therefore, a full tension SSC fitting upon completion has sufficient strength to satisfy the ANSI requirement of reaching $95 \%$ RBS. 



\section{SSC FEM THERMAL CYCLE SIMULATION}

\subsection{Thermal-Mechanical Evaluation for SSC Systems}

The source of long term thermal-mechanical associated damage to a SSC system is the net heat (Q) input into a SSC system, as illustrated in Figure 7-1. The net heat input is determined by: Qcurrent + Qsolar - Qradiation - Qconvection. Due to additional aluminum sleeve shielding at the SSC fitting region, the temperature profiles in the aluminum conductor strands within the SSC fitting is expected to be higher than that at the bare conductor region, especially after the thermal cycling. A detailed schematic diagram of SSC system is illustrated in Figure 7-2, where the SSC is divided into two sections, namely, core-grip and conduction sections; where the conduction section is responsible for transmitting electricity and the core-grip is responsible for secure the steel core.

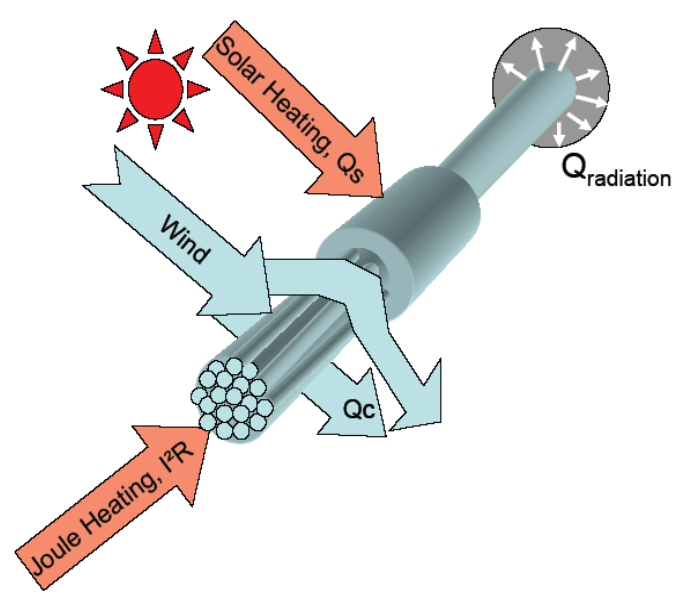

Figure 7-1 Net heat input.

The detailed cross-section to illustrate the material interfaces, between the splice sleeve and the conductors or between the steel core and the conductors, are shown in Figure 7-3. It is expected that the gap density at these interfaces will be increased as a result of thermal cycling events,

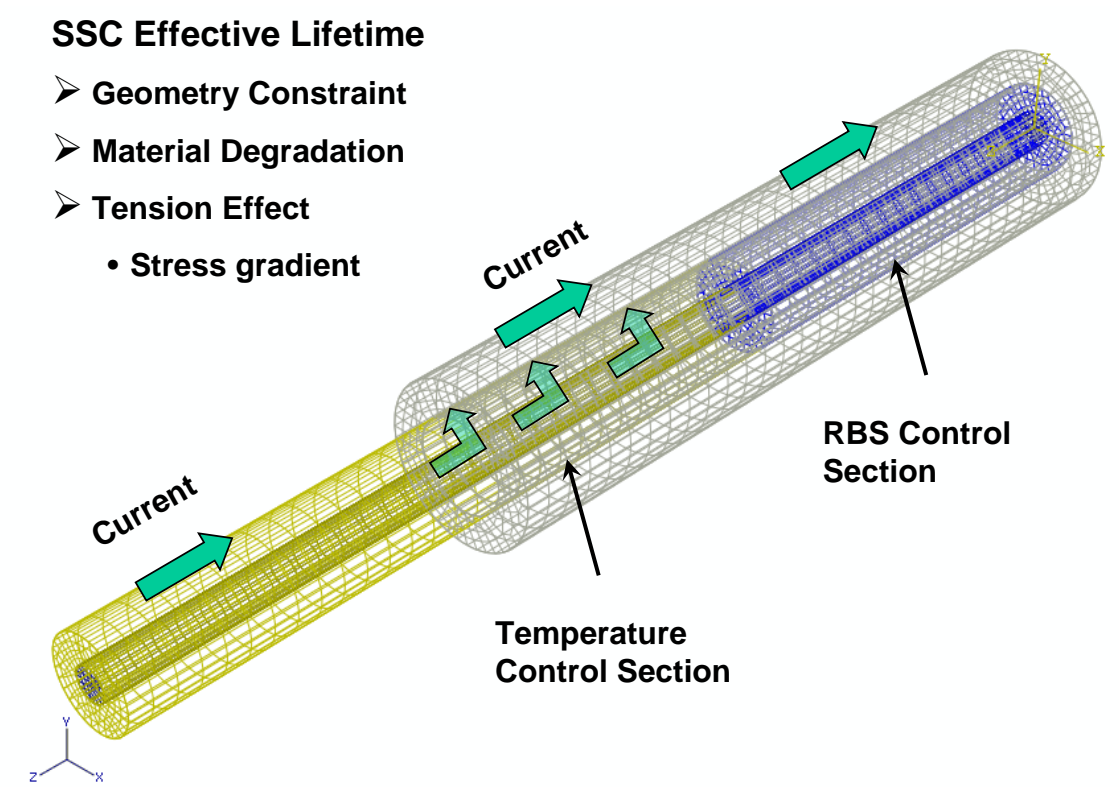

Figure 7-2 Schematic diagram of SSC system components and their functionalities. 


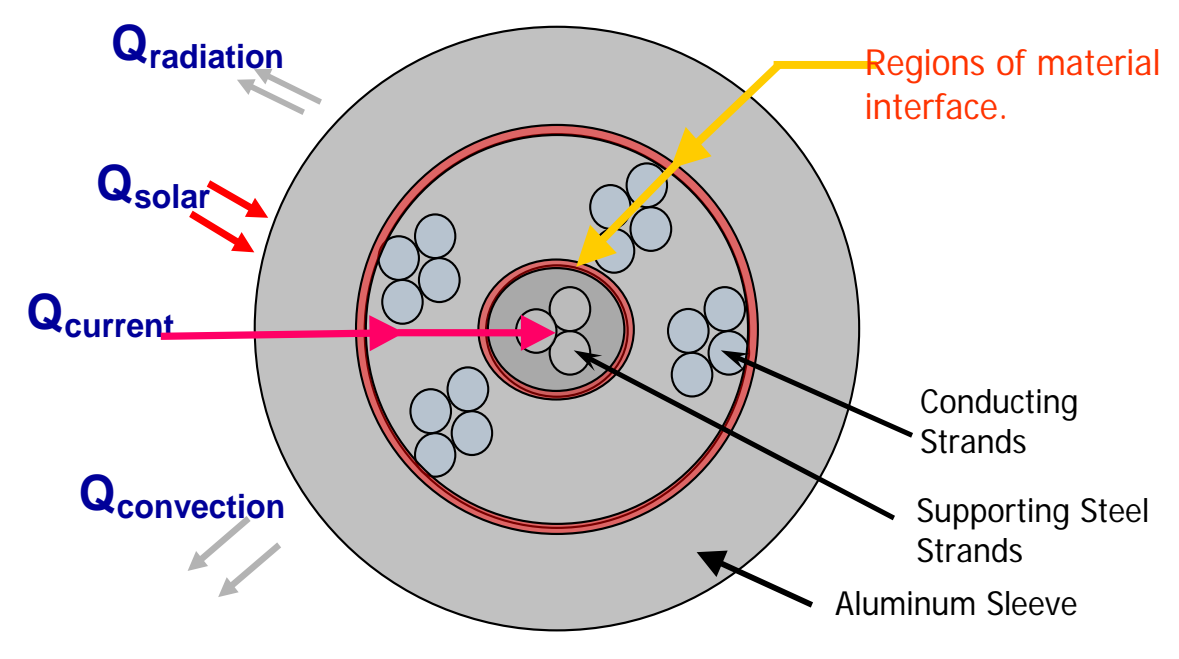

Figure 7-3 Schematic diagram of SSC system interfaces.

The consequence of reduced residual stress and the gap formation at these interfaces on the integrity and reliability of SCC, is two-fold

- $\quad$ Reduced electric and thermal conductivity, which could lead to higher local temperatures, accelerated aging and relaxation of the clamping stress

- $\quad$ Reduced connector clamping stress, which could ultimately lead to conductor separation and failure

\subsection{Temperature Profiles within TSC and SSC Fittings}

In collaboration with EPRI a series of experiments were carried out to determine the temperature profiles within the connector fitting of an ACSR Drake conductor system during thermal cycling. Measurements were obtained for conductors with both single- and two-stage connectors (TSC). Figure 7-4 shows the history of the ambient, conductor and fitting temperatures during a test (6 cycles per day) in which a reference temperature of $150^{\circ} \mathrm{C}$ was prescribed on the conductor surface about 5 meters away from the connector fitting, (SSC or TSC).

These experiments revealed that:

a) For a new system, SSC surface temperature is lower than the conductor surface temperature.

b) The temperature at the steel core is normally higher than that of aluminum wires, except at core-grip region.

c) The SSC splice surface temperature is lower than that of aluminum wires underneath.

d) The temperatures of a SSC system increased continuously during thermal cycling; temperature gradients observed from the embedded TCs in the SCC system also 
changed with thermal cycling. The majority of SSC conductor temperatures became greater than the $150^{\circ} \mathrm{C}$ reference temperature after about 50 cycles.

e) The significant temperature increases due to increased SSC resistivity are the direct result of the thermal cycle induced reduction in compressive residual stresses in the SSC system and the build up of aluminum oxide scale.

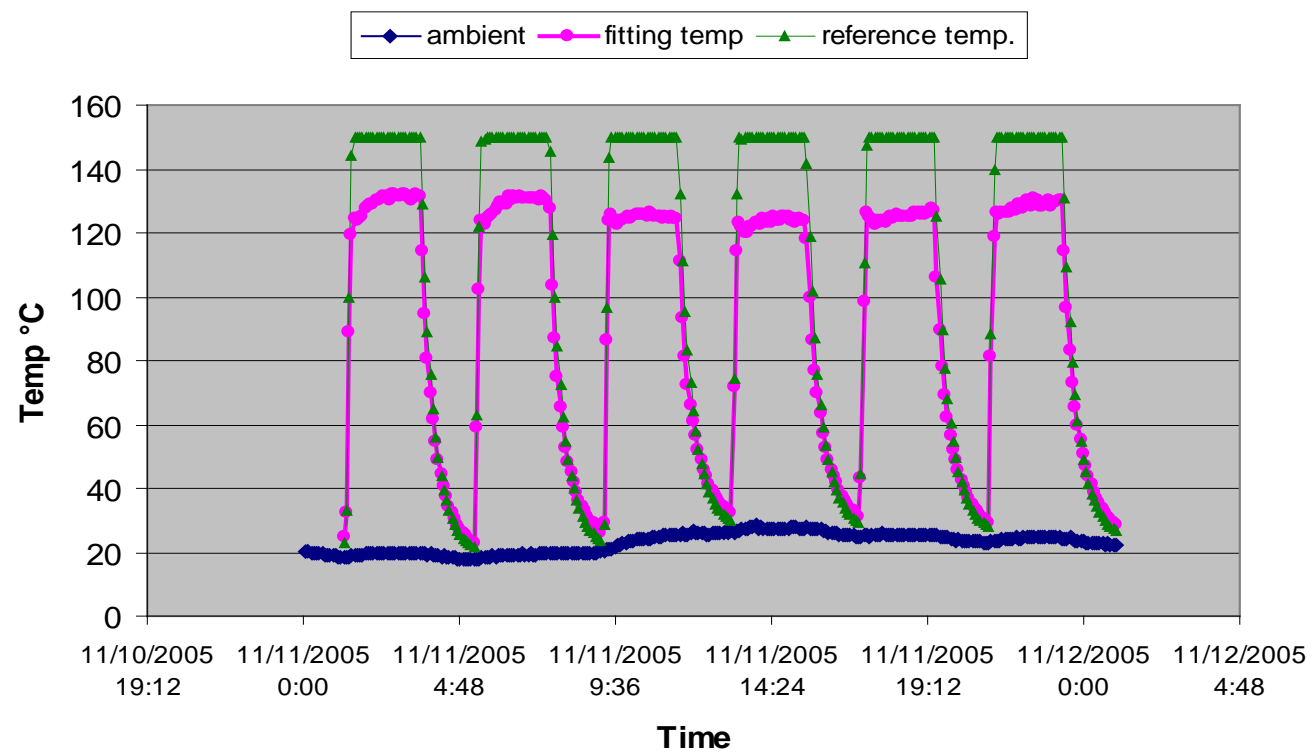

Figure 7-4 Typical thermal cycles of 6 cycles per day with target temperature of $150^{\circ}$

These experiments revealed that:

- For a new system, the connector surface temperature is lower than the conductor surface temperature.

- $\quad$ The temperature at the steel core is normally higher than aluminum wires, except at core-grip region of an SSC.

- The temperature of the SSC splice surface is lower than that of the aluminum wires underneath.

Table 7-1 Temperature profiles within SCC and TSC fittings

\begin{tabular}{|c|c|c|}
\hline $\begin{array}{c}\text { Location at } \\
\text { conductor } \\
\text { section }\end{array}$ & \multicolumn{2}{|c|}{ Temperature $\left[{ }^{\circ} \mathrm{C}\right]$} \\
\cline { 2 - 3 } & SSC & TSC \\
\hline Splice surf. & 92.6 & 79.7 \\
\hline Conductor surf. & 99.2 & 75.2 \\
\hline Steel core & 101 & 75.2 \\
\hline
\end{tabular}


- For a target conductor surface temperature of $150^{\circ} \mathrm{C}$, the temperature profiles of a SSC Drake conductor system on a fixed-ends frame with pre-tension of $6000 \mathrm{lb}$ at $20^{\circ} \mathrm{C}$, before and after 60 thermal cycles between $20^{\circ} \mathrm{C}$ and $150^{\circ} \mathrm{C}$, are listed in Table 7-2.

Table 7-2 Temperature profiles of SCC fitting

\begin{tabular}{|l|c|c|c|c|}
\hline \multirow{2}{*}{ Location } & \multicolumn{4}{c|}{ Temperature $\left[{ }^{\circ} \mathrm{C}\right]$} \\
\cline { 2 - 5 } & \multicolumn{2}{|c|}{ Conductor section } & Core-grip section \\
\cline { 2 - 5 } & Initial & $>60$ cycles & Initial & $>60$ cycles \\
\hline Splice surf. & 90 & 152 & 90 & 152 \\
\hline Conductor / or Core-grip surf. & 97 & 164 & 94 & 177 \\
\hline Steel core & 99 & 166 & 92 & 166 \\
\hline
\end{tabular}

- These results indicate that thermal cycling leads to a significant increase in the temperature within the SSC system. Furthermore, the maximum temperature per cycle at interface between aluminum wire and splice sleeve from 10 SSCs located in 4 conductor spans are shown in Figure 7-5. The temperatures increased continuously during thermal cycles, with different rates revealed from the 10 thermocouples (TC), and the majority of TCs became greater than the $150^{\circ} \mathrm{C}$ reference target temperature after about 50 cycles. It is noted here that splice of TC-53 was replaced after 45 cycles, and TC- 117 partially lose contact with the splice during 30 to 45 thermal cycles.

- The significant temperature increased in SSC system shown above is the direct results of reduction of thermal and electric conductivity in the SSC system. The thermal cycle induced reduction in compressive residual stresses and the potential of the gap formation at interface in the SSC system, in addition to the aluminum oxide scale built up, could be the root causes.

- Water quench was applied once per100 thermal cycles to half the 8 testing spans. Significant thermal spike phenomena were observed from the quench span but not from the non-quench span. Figure 7-6 and Figure 7-7 present a comparison of quenched and nonquenched data, at the interface between steel core and aluminum conductor. Significant drop in tension loading were observed as noted results from four SSC-spans shown in Figure 7-8. This phenomenon indicates that the progressive slippage of conductor wire was occurring in the SSC system; this is the direct result of losing compressive residual stresses in the SSC system due to thermal cycling. 


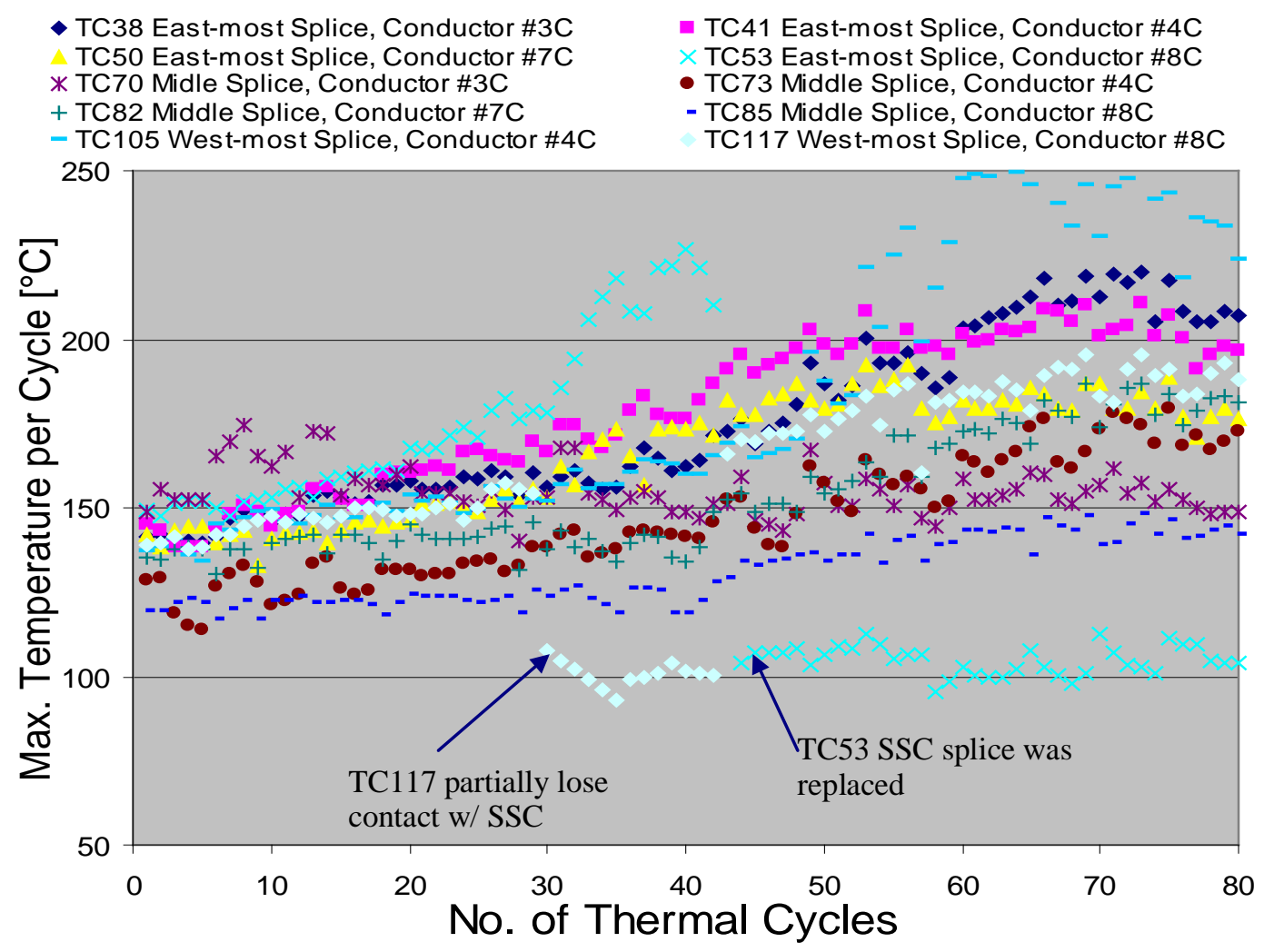

Figure 7-5 Maximum temperatures at conductor/splice sleeve interface per cycle within ACSR SSCs measured from 10 thermocouples, with target conductor surface temperature of $150^{\circ} \mathrm{C}$ at 15 feet away from the SSC. Temperature increased dramatically in ACSR SSC system during thermal cycles.

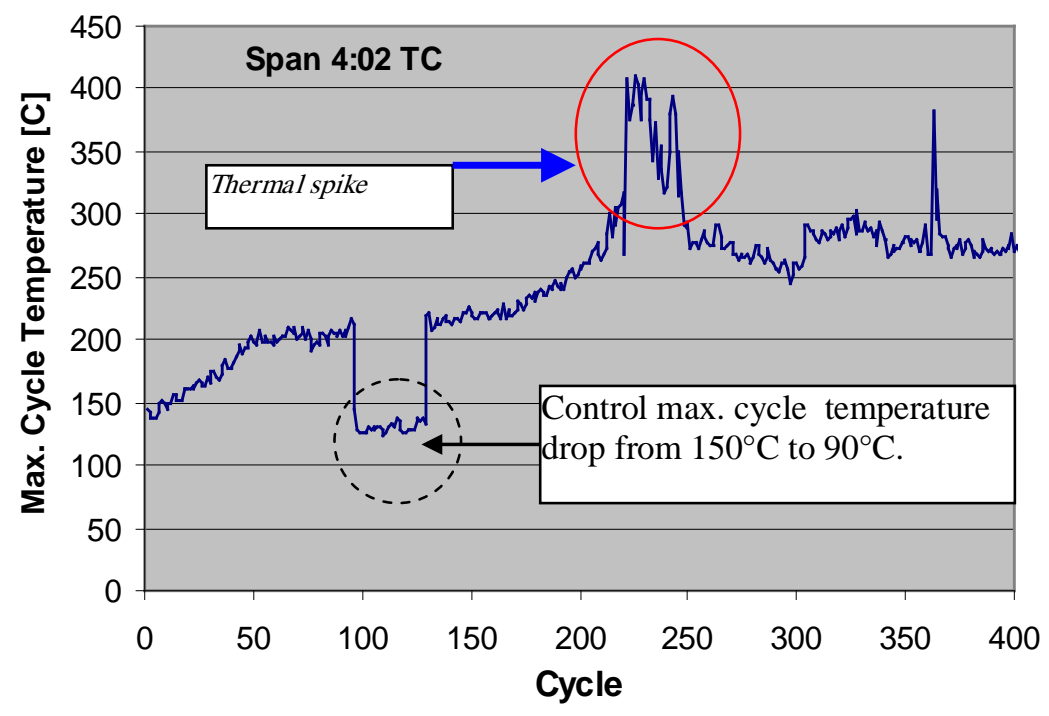

Figure 7-6 Maximum cycle temperature history for quenched data. 


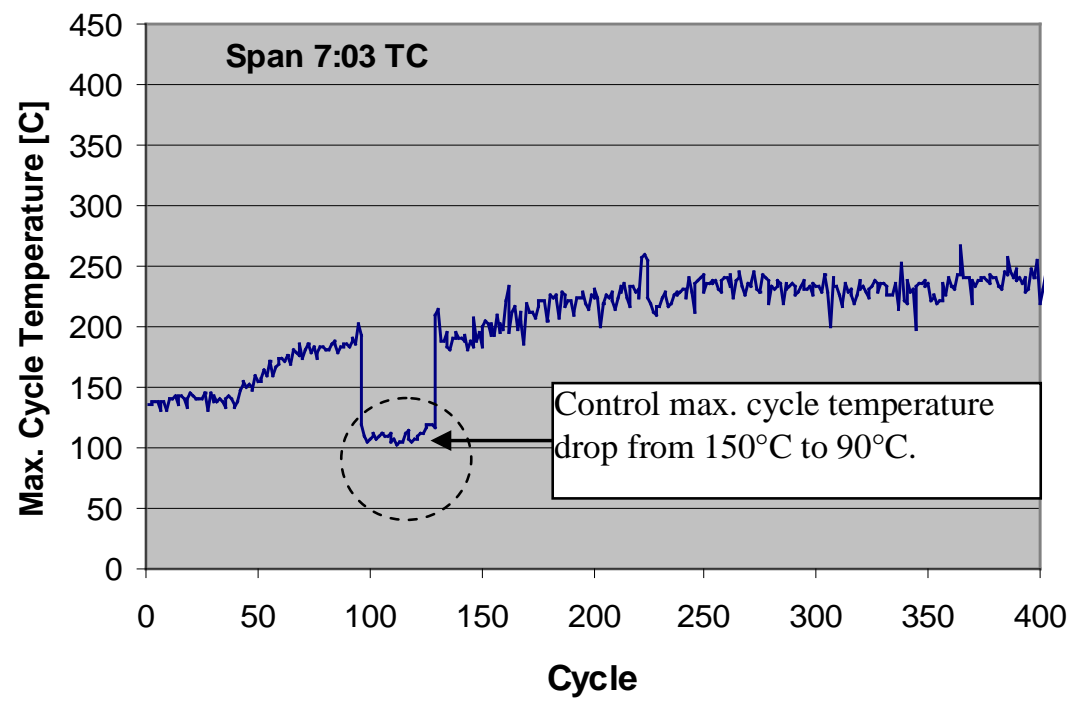

Figure 7-7 Maximum cycle temperatures for non-quenched data.

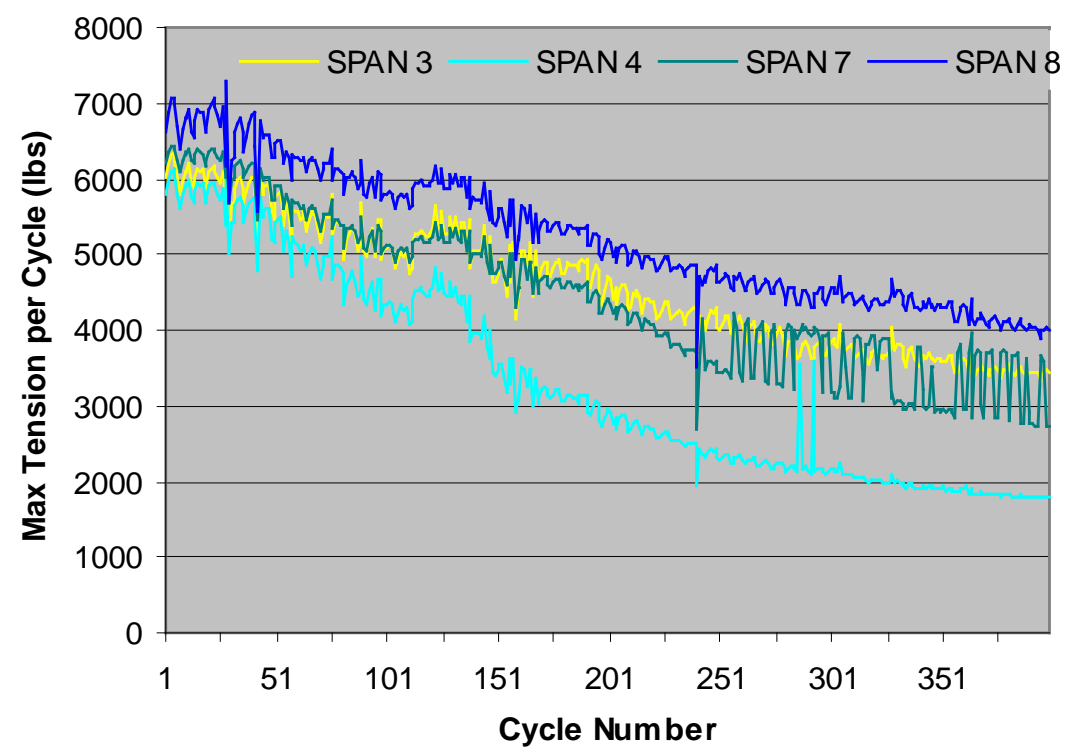

Figure 7-8 Significant decrease in tension lading of the test spans that contains SSC fittings $\mathrm{w} /$ initial tension of $\sim 6000 \mathrm{lb}$.

f) The SSC temperature profiles obtained from thermal cycling tests are used as temperature input for FEM thermal cycle simulations, where heat-up sleeve surface temperature are at $70^{\circ} \mathrm{C}, 100^{\circ} \mathrm{C}, 125^{\circ} \mathrm{C}, 150^{\circ} \mathrm{C}, 175^{\circ} \mathrm{C}, 225^{\circ} \mathrm{C}, 250^{\circ} \mathrm{C}, 275^{\circ} \mathrm{C}, 290^{\circ} \mathrm{C}, 315^{\circ} \mathrm{C}$ and $350^{\circ} \mathrm{C}$, and the cool-down temperature is at $20^{\circ} \mathrm{C}$, as shown in Figure 7-9 and Figure 7-10, for the conductor and the core-grip sections, respectively. 


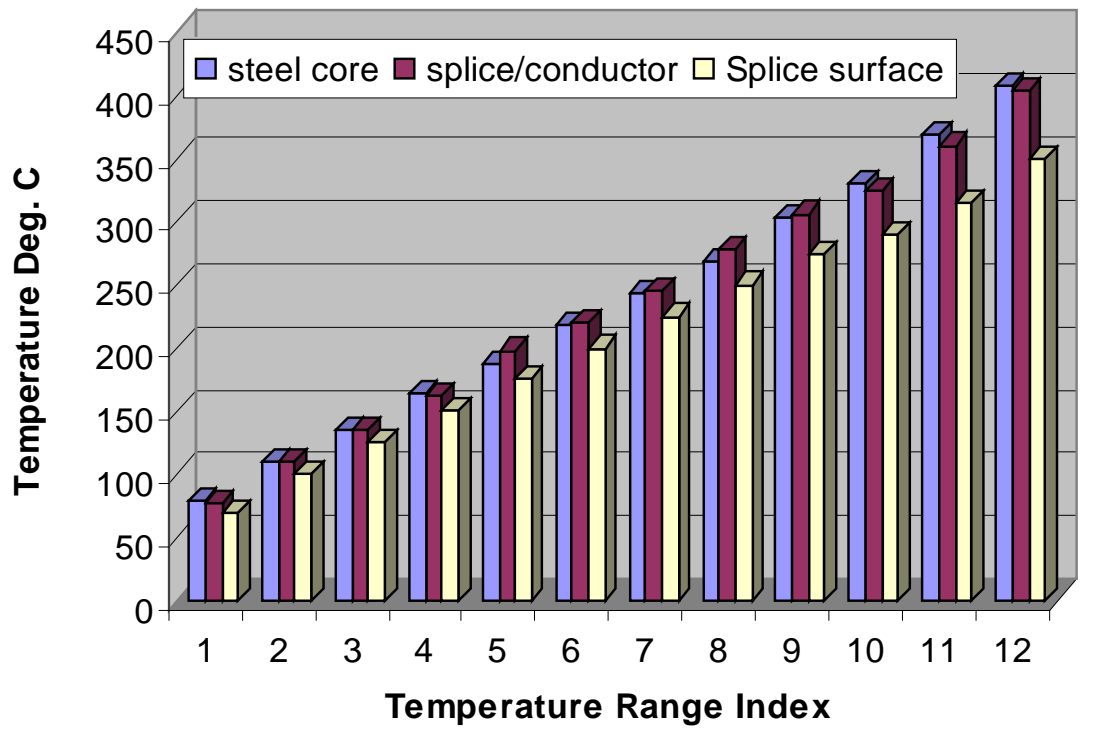

Figure 7-9 Temperature profile history of conductor section in SSC system used for FEM thermal-cycle simulation.

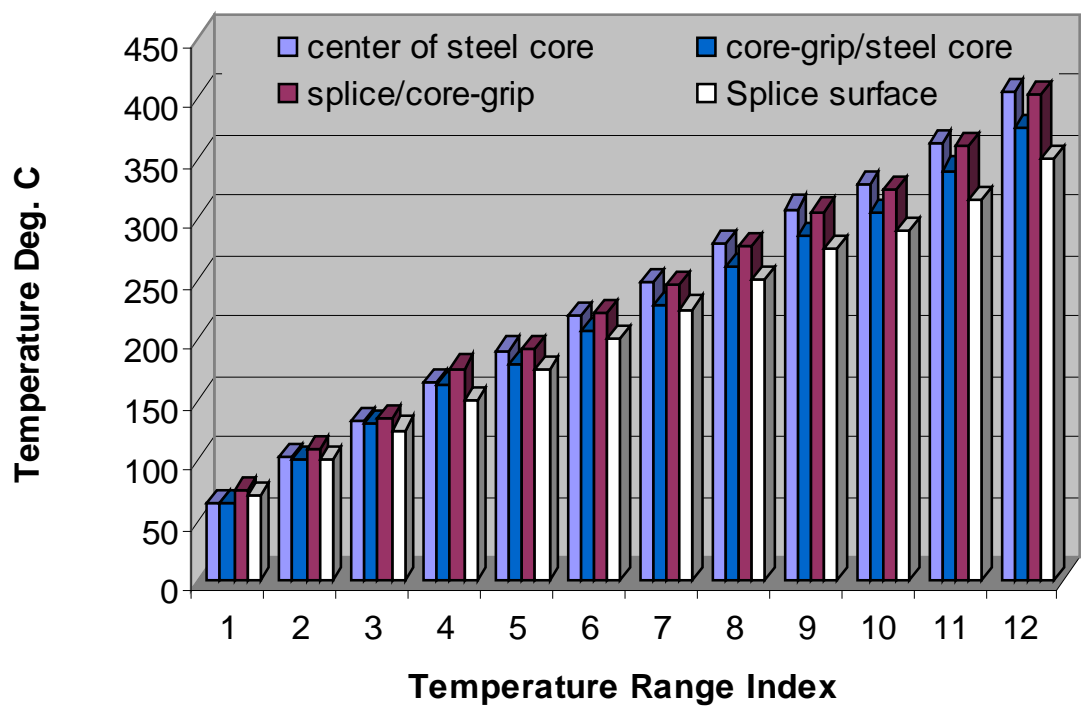

Figure 7-10 Temperature profile history of core-grip section in SSC system used for FEM thermal-cycle simulation.

\subsection{SSC FEM THERMAL CYCLING SIMULATION}

The FEM thermal cycle simulations for SSC system were carried out sequentially for (heat-up) temperature ranges from $70^{\circ} \mathrm{C}, 100^{\circ} \mathrm{C}, 125^{\circ} \mathrm{C}, 150^{\circ} \mathrm{C}, 175^{\circ} \mathrm{C}, 225^{\circ} \mathrm{C}, 250^{\circ} \mathrm{C}, 275^{\circ} \mathrm{C}, 290^{\circ} \mathrm{C}$, $315^{\circ} \mathrm{C}$ and $350^{\circ} \mathrm{C}$ (based on the splice surface temperatures),; and with cool-down temperature of $20^{\circ} \mathrm{C}$. There are three-interaction cycles for each target temperature range. The parameter 
studies for tensile loading effect and the quench effect were also carried out. The center section of simulated SSC FEM model was used for compressive residual stress degradation study of an ACSR Drake conductor SSC system, as shown in shaded area of crimped section in Figure 7-11. In the FEM model, the right end of the ACSR Drake aluminum conductor, including steel core, was fixed and no constraints were set for the left end portion of aluminum conductor. In order to investigate tension effect on SSC thermal cycling, two load boundary conditions were considered: (1) no tension loading and (2) with 25\% RBS tension loading. The measured temperature profiles were incorporated into the FEM simulated SSC system during FEM thermal cycling simulation.

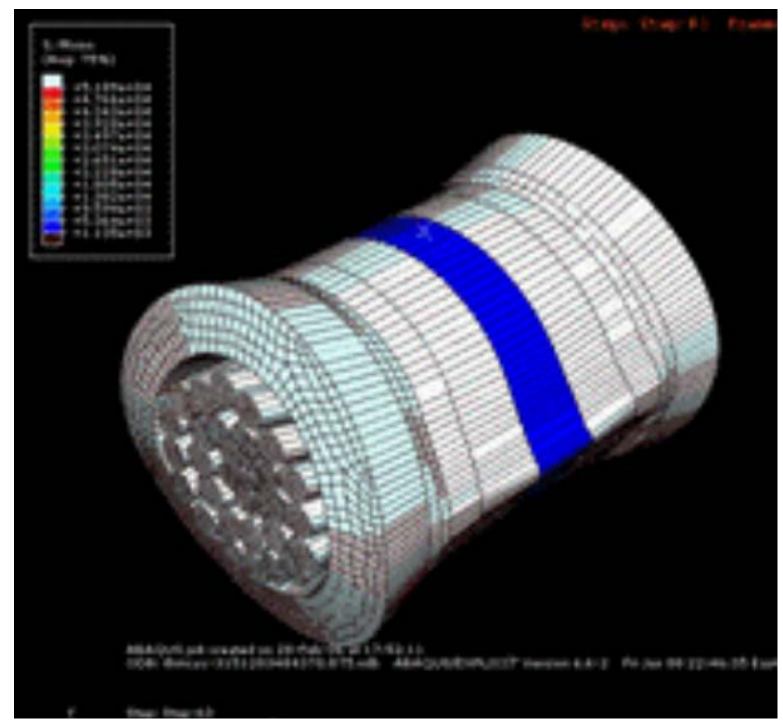

Figure 7-11 FEM simulated SSC system.

The load distributions between aluminum wires and steel core wires used for FEM thermal cycle simulation were referred to tension data listed in Table 2-4 of Southwire Overhead Conductor Manual for a 600-ft span ACSR Drake conductor cable under heavy load conditions [2]. At knee point of $100^{\circ} \mathrm{C}$, the steel-core wires will carry the entire cable tensile load. The tensile stress distributions among steel and aluminum wires of a 600-ft span Drake conductor are shown in Table 7-3.

Table 7-3 Stress distribution for 600-ft span Drake conductor

\begin{tabular}{|c|c|c|}
\hline & \multicolumn{2}{|c|}{ Tensile Stress [psi] } \\
\hline Temp. & Steel & Al. \\
\hline $20^{\circ} \mathrm{C}$ & 33,340 & 7,147 \\
\hline $45^{\circ} \mathrm{C}$ & 31,422 & 4,197 \\
\hline $70^{\circ} \mathrm{C}$ & 29,504 & 1,247 \\
\hline$\geq 100^{\circ} \mathrm{C}$ & 31,875 & 0 \\
\hline
\end{tabular}

FEM thermal cycle simulation results show different temperature sensitivity between core-grip and conductor sections, where the conductor section shows significant reduction in the 
compressive stress in SSC system when the thermal cycle temperature reached $125^{\circ} \mathrm{C}$, where the core-grip section still has significant compressive strength up to $200^{\circ} \mathrm{C}$.

\subsubsection{Thermal Cycle Simulation for SSC Conductor Section}

The FEM simulation was performed by utilizing 3-D dynamic/inelastic modeling with "general contact” algorithm. A material property table for SSC components was generated to serve as input for FEM analyses, which contains four major parameters, namely, temperature, material strength (yield, young's module, etc.), material threshold for heat-up or cool-down cycle, and the inelastic stress-strain curves. The 10 hour holding-time stress-strain curves were used in the FEM thermal cycle simulation investigation.

Simulation without tensile loading shows much less effect of compressive residual stress degradation due to thermal cycling; and no significant reduction, both in compressive strength at cool-down/ambient and heat-up temperatures, till $250^{\circ} \mathrm{C}$ thermal cycling range. The FEM results with tension loading also show that significant voids or gaps exist for simulation, while the closed pack formation of the conductor wires formed at baseline largely remained intact for notension FEM simulation, even at $315^{\circ} \mathrm{C}$ thermal cycle range, as shown in Figure 7-12.

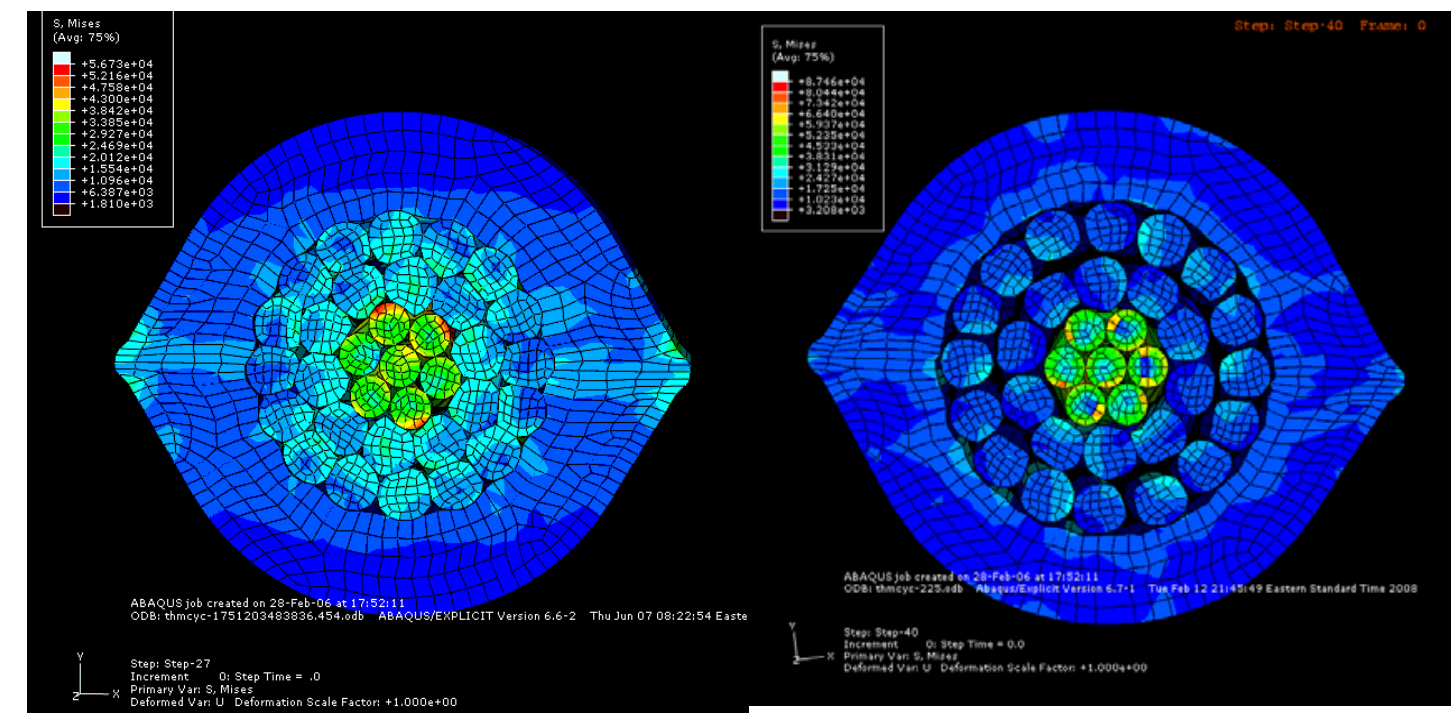

Figure 7-12 FEM thermal cycle simulation (left) at $315^{\circ} \mathrm{C}$ cycle range no tension loading, (right) at $225^{\circ} \mathrm{C}$ cycle range with tension loading.

- Significant relative movement of conductor wire and steel- core within SSC system was observed from thermal cycle simulation with tension loading; such phenomenon was not observed for thermal cycling simulation without tension loading, as shown in Figure 7-13. Where the marked center section of the SSC remains in the original order for non-tension simulation, but significant relative movement or disorder of the marked (or shaded) center section was observed for tension simulation. This is a direct indication that the SSC system has lost its grip strength and conductor slip has occurred. 


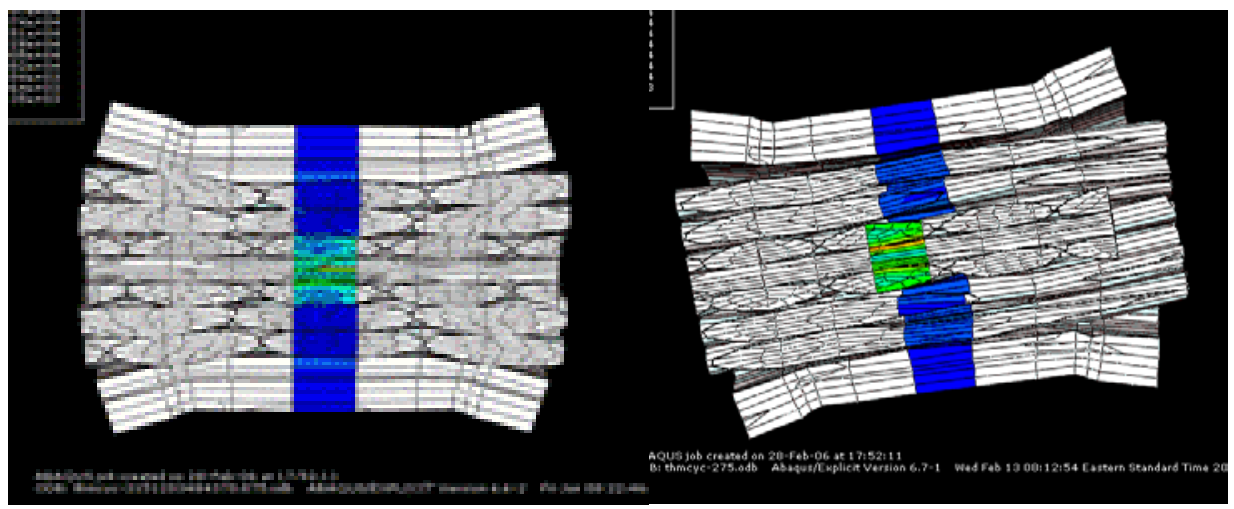

Figure 7-13 (left) without tension simulation at $315^{\circ} \mathrm{C}$ thermal cycling range, and (right) with tension at $275^{\circ} \mathrm{C}$ thermal cycling range.

- Simulation with tension or/and quench show severe reductions of SSC compressive stress fields, both in heat-up and cold down cycles, at or above thermal cycle range of $100-125^{\circ} \mathrm{C}$, where 92-95\% reduction was reached. The FEM simulation results with quench and tension at $225^{\circ} \mathrm{C}$ thermal cycle is shown in Figure 7-14, where the majortry of conductor wires lost contact with each other and the SSC sleeve.

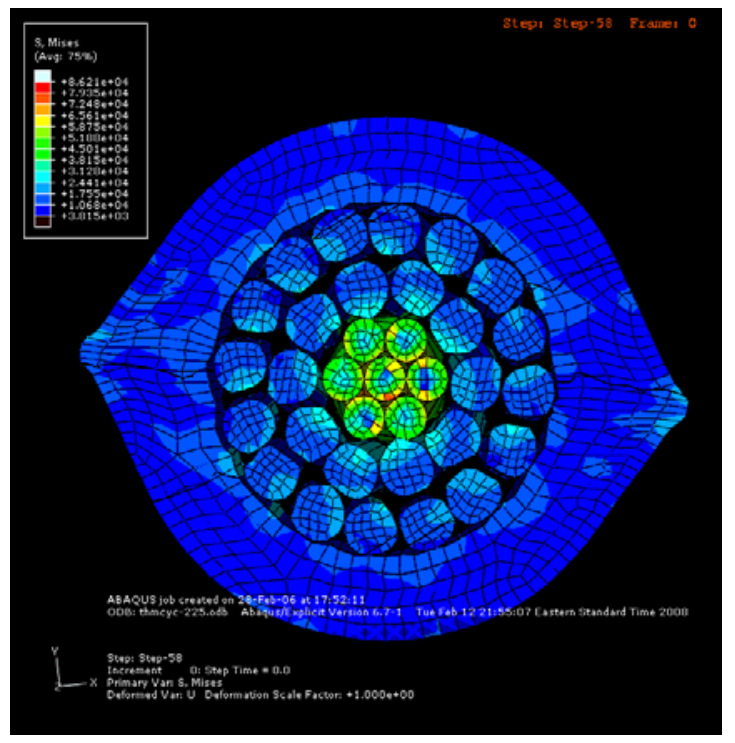

Figure 7-14 FEM Simulation at $225^{\circ} \mathrm{C}$ with quench and tension loading applied.

- The compressive force applied to the aluminum conductor boundary at heat-up and at cooldown (room-temperature) thermal cycles are shown in Figure 7-15 and Figure 7-16, respectively. During simulation, three occurances of heat-up/cool-down thermal cycles were calculated at each target temperature range before FEM simulation moves on to the next higher temperature range. Significant degradation of the compressive force in SSC was observed from both heat-up and cool-down cycles. It appears that at the end of third iteration of the $70^{\circ} \mathrm{C}$ thermal cycle, the SSC has lost about $70 \%$ compressive strength compared to that at the initial stage, $20^{\circ} \mathrm{C}$. The quench plus tension case appears to be consistent with that of 
full tension case, except at $125^{\circ} \mathrm{C}$, which is illustrated in Figure $7-17$. The half-tension $(12 \%$ RBS) and half-tension plus quench cases show similar behavior to that of full tension case.

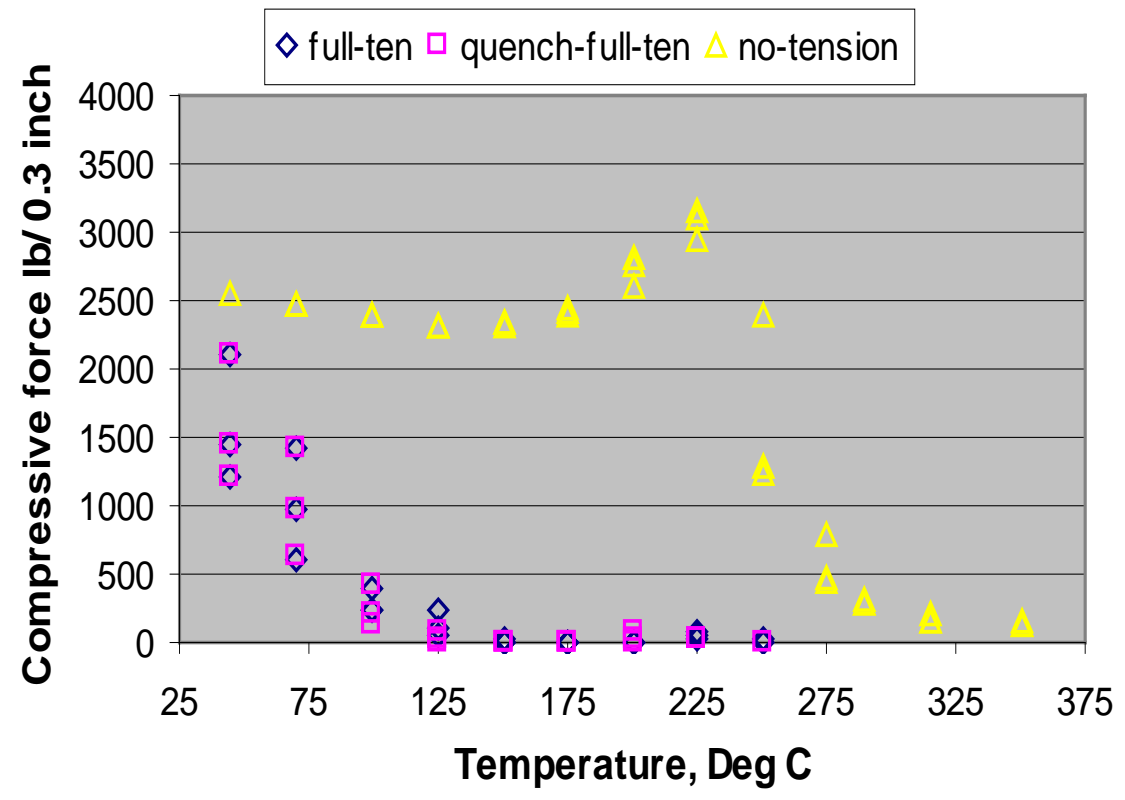

Figure 7-15 The compressive force in conductor section at heat-up cycles

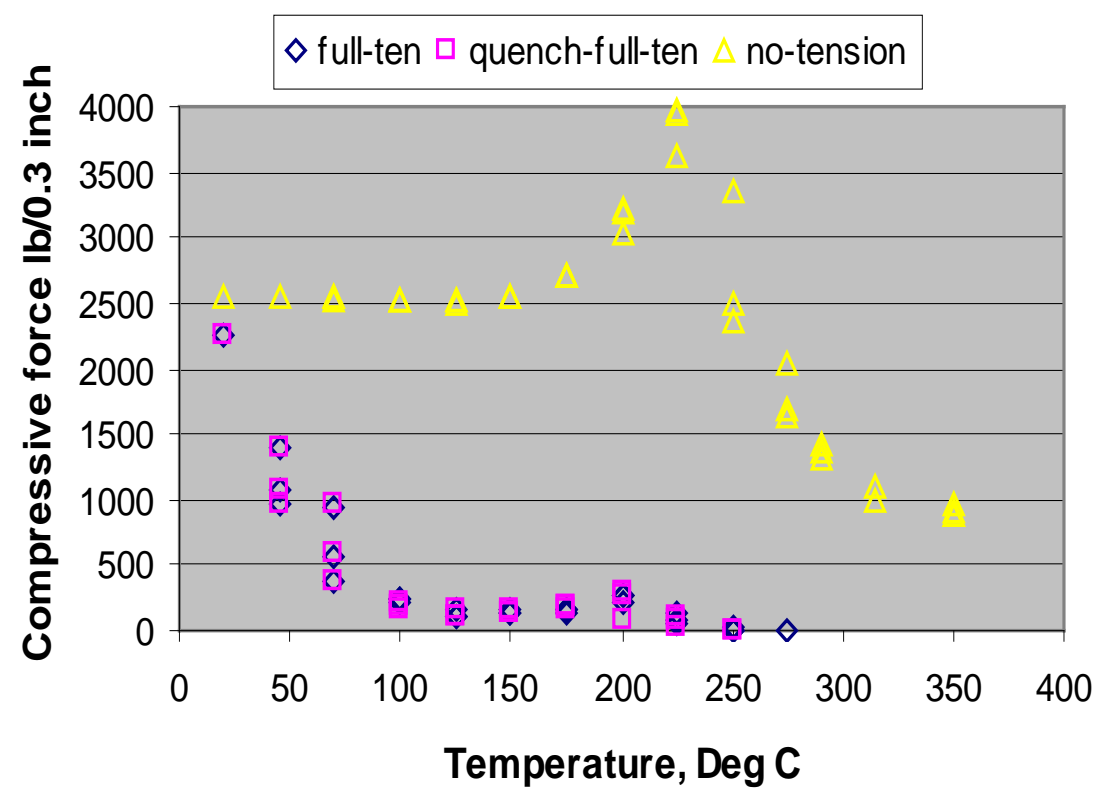

Figure 7-16 Compressive force in conductor section at cool-down. 


\subsubsection{Thermal Cycle Simulation for Core-Grip Section}

2-D plane stress quasi-static/inelastic model with "contact pairs" algorithm was used for thermal cycle simulation. The axial tension loading of core-grip section was simulated with lateral contraction of steel-core wire at the corresponding tensile loading and temperature. The FEM simulation indicates that the contact area between the core-grip and the steel core are quite limited, as shown in Figure 7-18. The FEM simulation indicates that the compressive force around the steel core decreases continuously during thermal cycling. The discussions of FEM simulation results are described below.

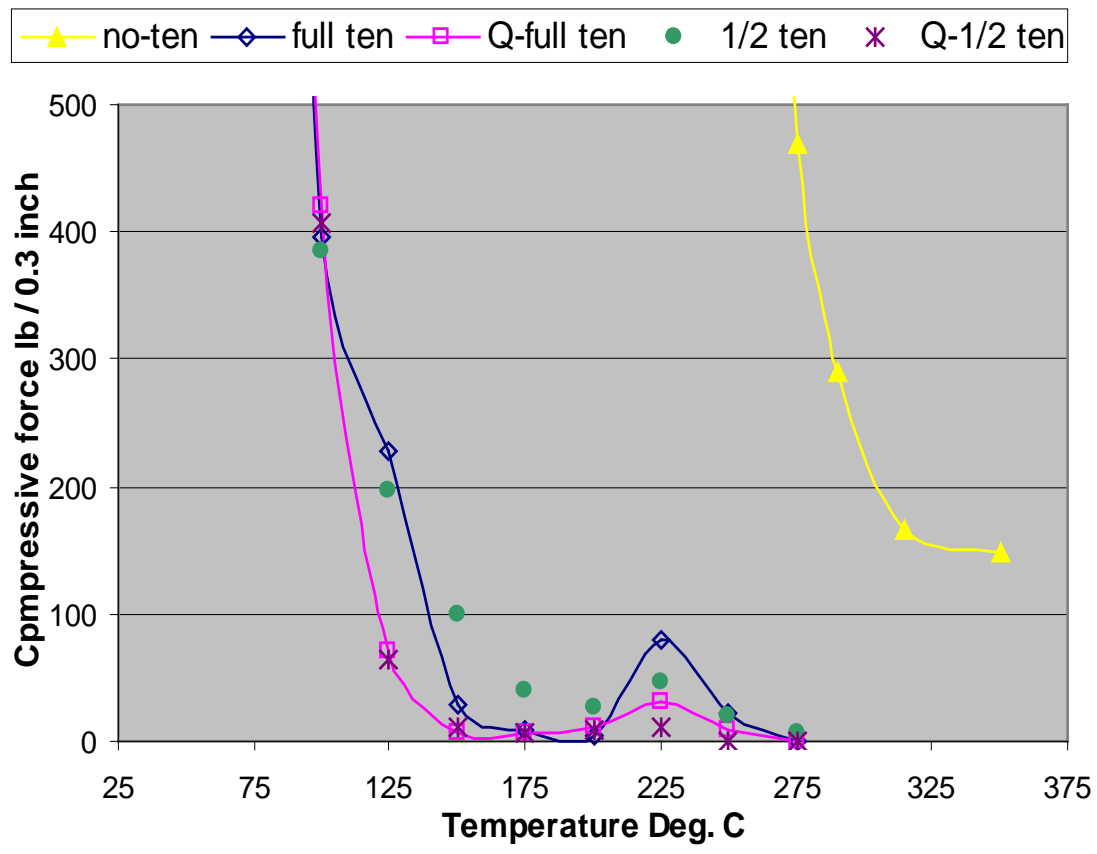

Figure 7-17 Enlarged section view of compressive force in conductor section at the first iteration during heat-up cycle.

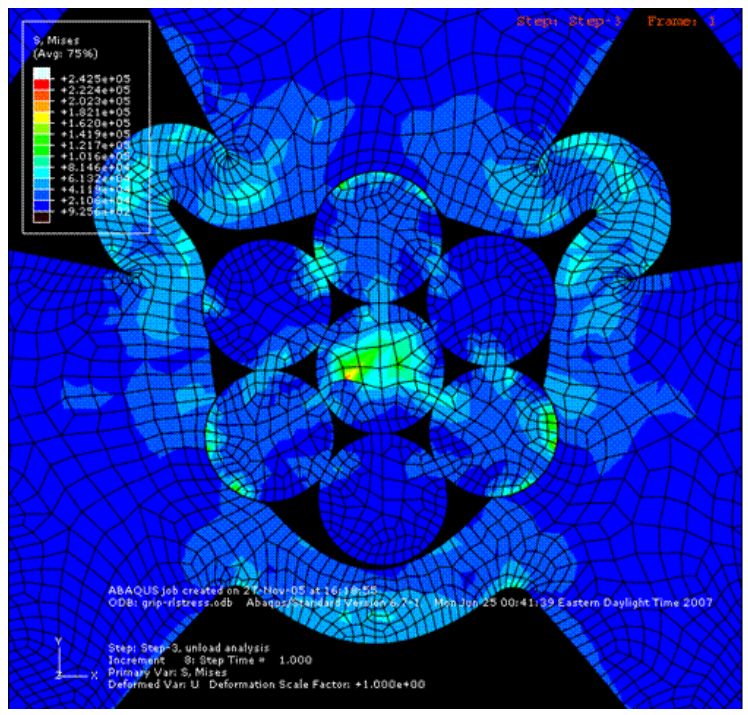

Figure 7-18 FEM model of SSC core-grip section center at steel core. 
- No significant difference in the compressive stress trend was found from thermal cycle simulation with tension or without tension loading. But different trends were observed between compressive force degradation at heat-up and cool down cycles. As illustrated in Figure 7-19 and Figure 7-20, where the cool-down/room-temperature compressive force is degraded much slower than the counterpart at heat-up cycle at targeted thermal cycle range. Based on this finding, the current ANSI requirement of using room temperature tensile testing for evaluating the SSC reliability after thermal cycling needs to be modified to incorporate the tensile testing at the heat-up cycle of the targeted thermal cycling temperature.

- The compressive forces of core-grip section decrease much less than that of conductor section during thermal cycling. For instance, under full tension at $150^{\circ} \mathrm{C}$ heat-up cycle, the compressive force of conductor section has already reduced to near zero, while for the core-grip section it still has about 50\% compressive force left. The rapid decrease of compressive force in conductor section at the early thermal cycle stage, from $20^{\circ} \mathrm{C}$ to $125^{\circ} \mathrm{C}$, means that the SSC system will have a relatively sharp increase in temperature at this temperature range and then approach a quasi-steady state. This is consistent with that observed from the thermal cycle temperature profile experiment. It is noted here that the core-grip section lost its compressive force completely at about the $300^{\circ} \mathrm{C}$ temperature cycle.

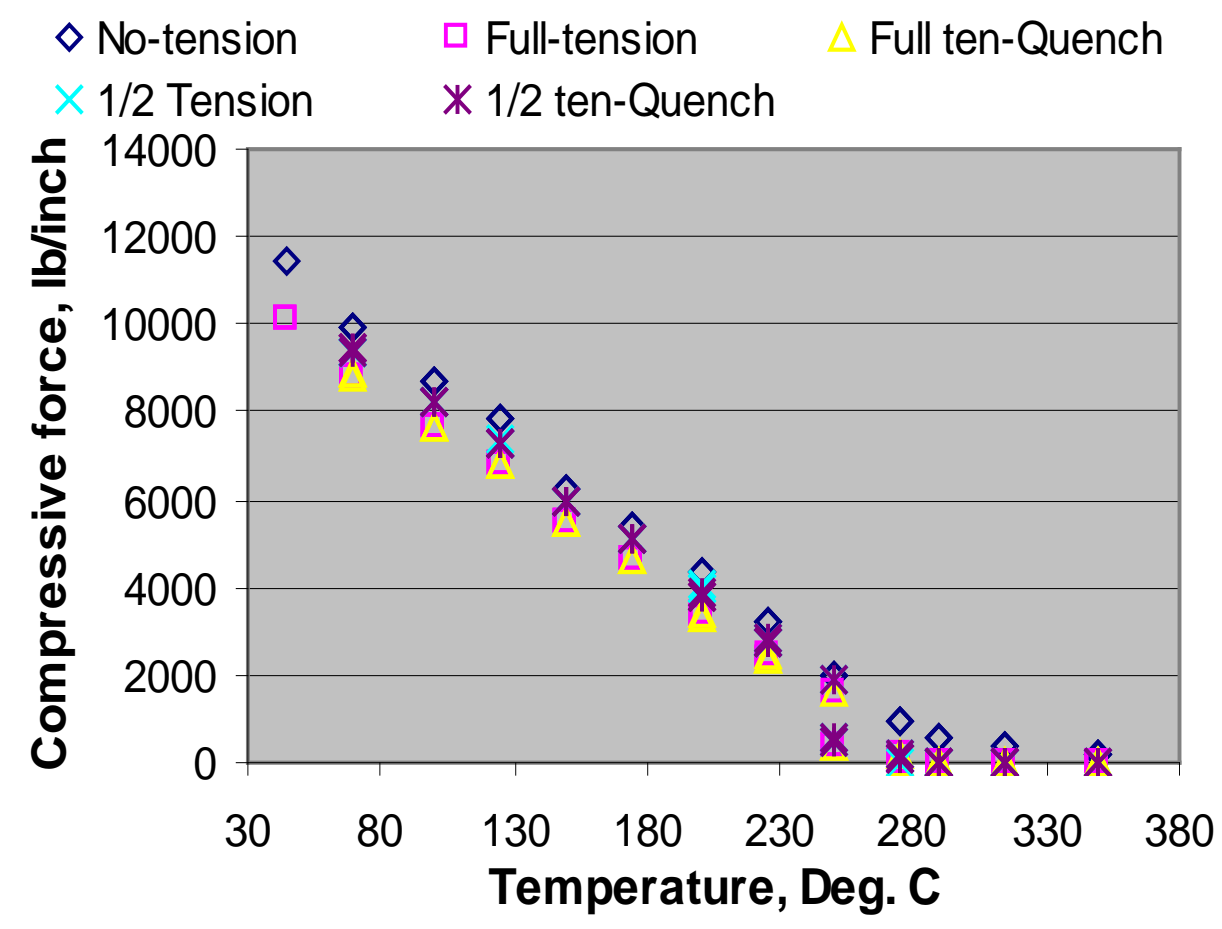

Figure 7-19 Compressive force around steel-core at heat-up cycle. 


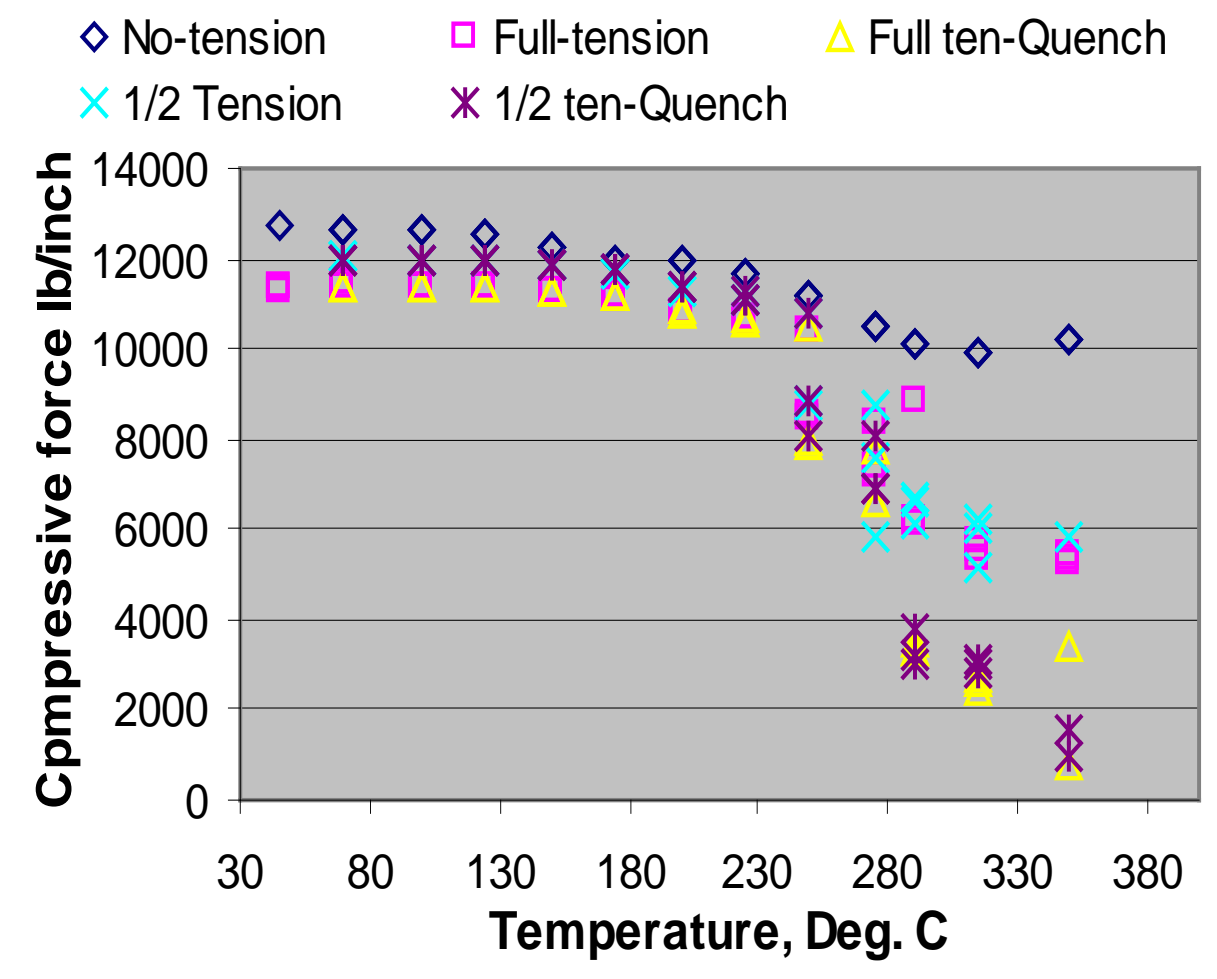

Figure 7-20 Compressive force around steel-core at cool-down.

\subsubsection{Consideration of Single Iteration per Target Temperature}

The FEM simulation results based on multiple iterations per target temperature approach indicate that the compressive stress will continuously decrease for one target temperature during multiple iteration cycles. The reduction of compressive stress will result in increasing resistivity of the SSC; thus, the increase of SSC system temperature. Therefore, in reality the follow-on iteration cycle will not likely keep the same temperature as that of the earlier iteration cycle within one target temperature cycle, except with the reduction of the electric current. Such phenomenon was also observed from the continuing increase of the maximum cycle temperature from thermal cycling experiments. Thus, the data obtained from the multi-iterations algorithm may further strengthen the concept of thermal cycling effect, but when it combined with the "sequential temperature increase” algorithm for thermal cycle simulation will result in overly conservative estimates for SSC effective lifetime investigation. It is noted here that the simulation result of the conductor section seems to be very sensitive to the multi-iteration algorithm, while the coregrip section does not, except for cool-down data at higher temperature (above $250^{\circ} \mathrm{C}$ ). Based on above, "Single cycle iteration per target temperature" algorithm was adopted to reproduce the simulation result for conductor section shown in Figure 7-21 and Figure 7-22, and for core-grip section at temperature above $250^{\circ} \mathrm{C}$, shown in Figure $7-23$ and Figure $7-24$. These FEM simulation results were further used in the SSC effective lifetime estimates. 


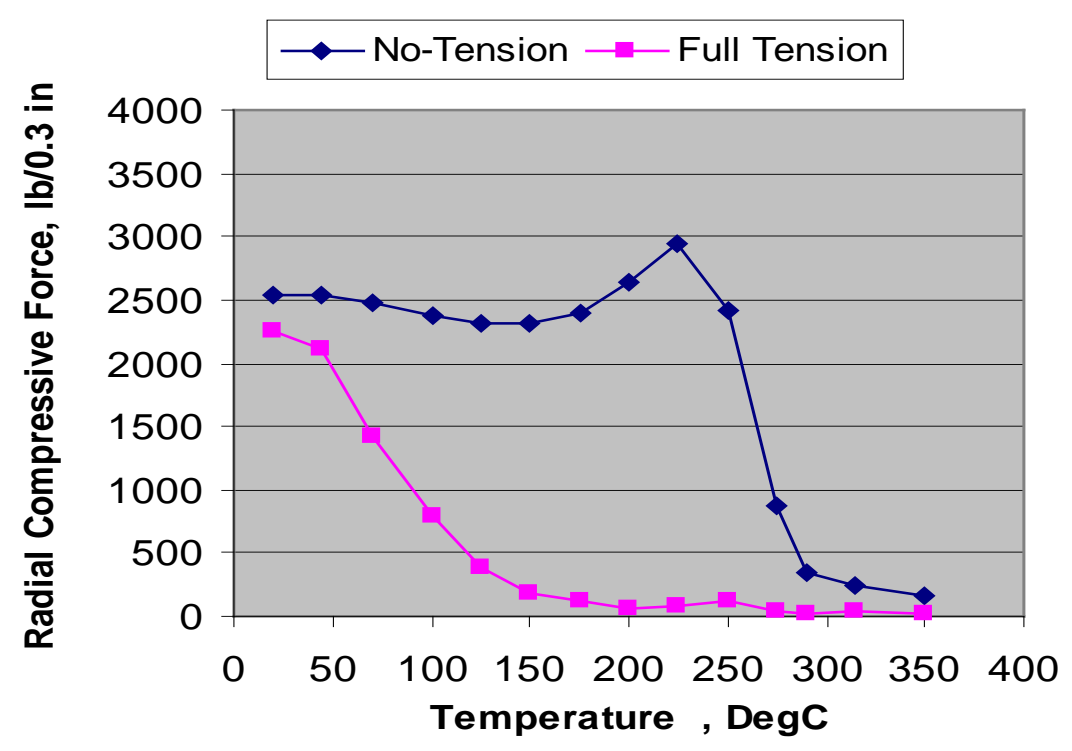

Figure 7-21 Compressive force in conductor section at heat-up.

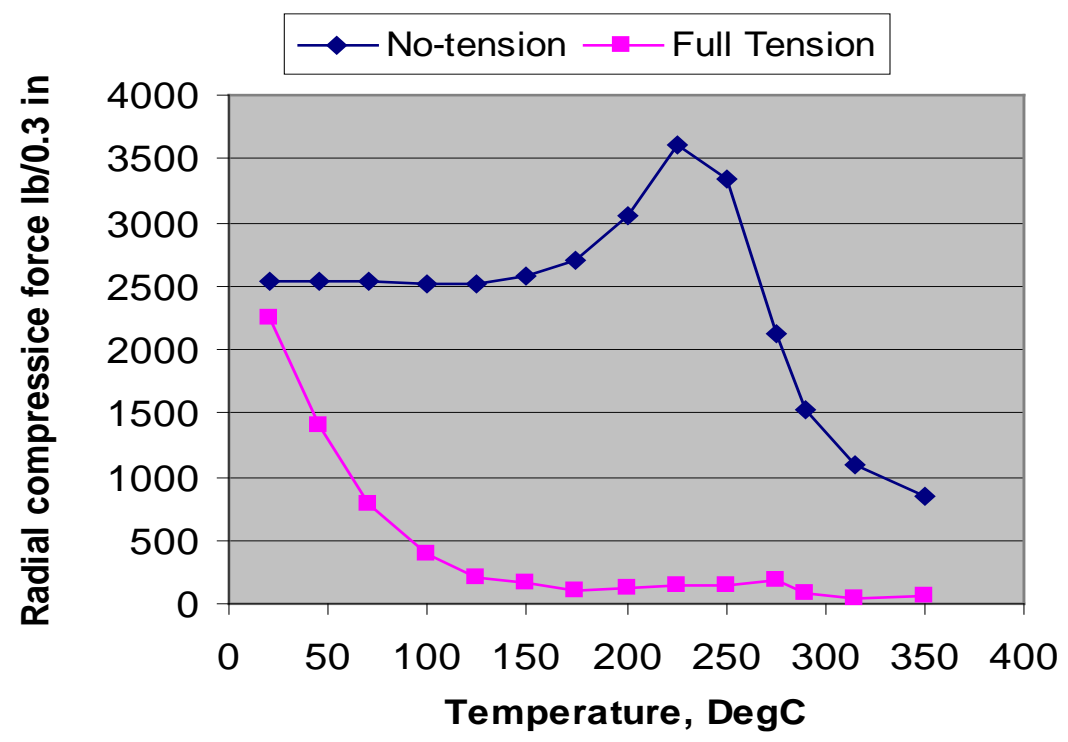

Figure 7-22 Compressive force in conductor section at cool-down. 


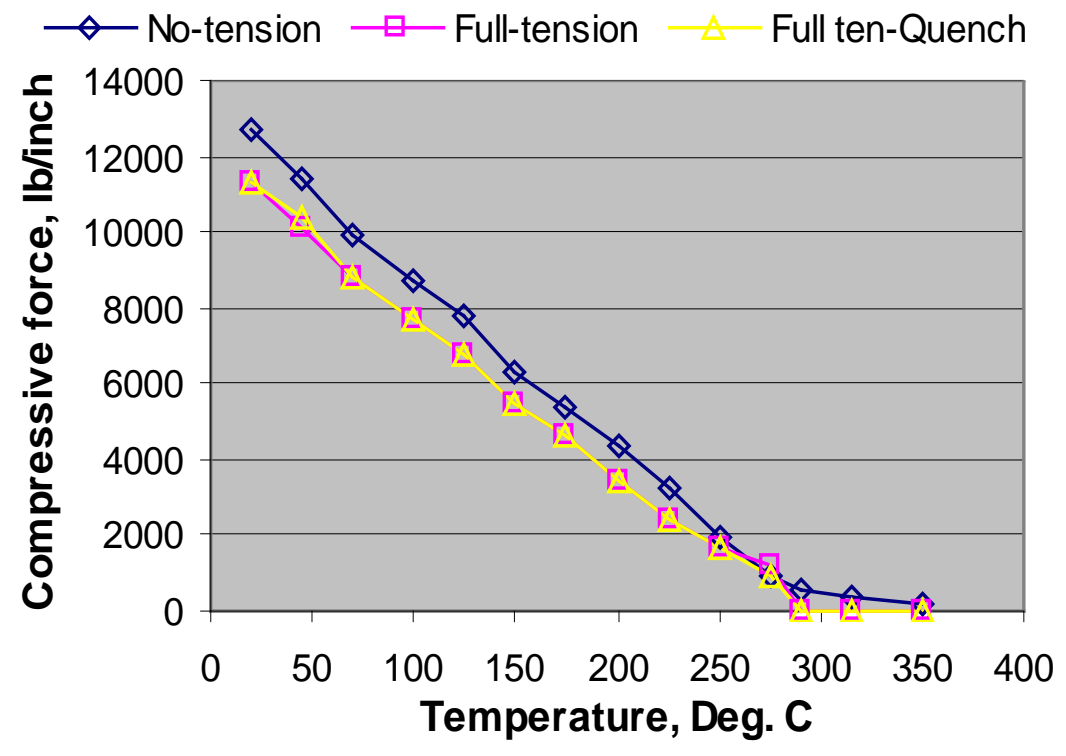

Figure 7-23 Compressive force around steel-core at heat-up.

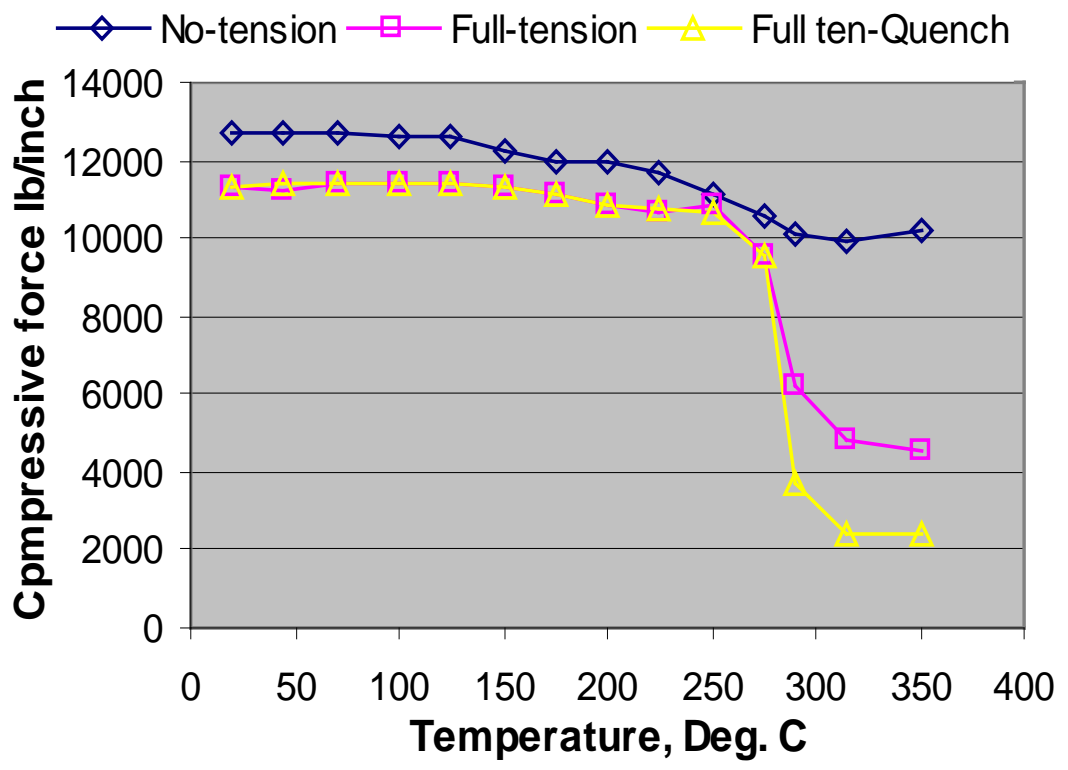

Figure 7-24 Compressive force around steel-core at cool-down. 


\section{$\boldsymbol{8}$ sSC EFFECTIVE LIFETIME ESTIMATE}

The accelerated connector aging can be attributed to connector component material aging, including creep, fatigue and TMF, and aluminum oxide film build-up and microstructure evolution at the interfaces within the connector system. This study further verifies that significant degradation of connector compressive residual stress will occur during service, especially at higher operation temperature. The consequences of degradation of compressive stress field are an increase of SSC system resistivity and the reduction of SSC initial rate tensile strength that was evaluated according to ANSI C119.4. Therefore, the compressive residual stress within a SSC can serve as a good indication to project the effective lifetime of a SSC conductor system during service.

According to extensive thermal mechanical test results for SSC components, we have drawn the following conclusions:

- Creep data has very low and stable creep strain at relatively low temperature $\left(\sim 70^{\circ} \mathrm{C}\right)$ and medium loading for the SCC aluminum materials. For conductor at temperatures above the knee point, aluminum conductor wires will relax and have zero tensile loading, and the associated long term creep strain is negligible due to lack of axial tensile loading.

- From fatigue point at isothermal condition, due to relatively constant conductor cable axial loading, the associated fatigue damage to the SSC system is considered secondary. Based on thermal-mechanical fatigue data, the TMF associated strain hardening was balanced by the thermal annealing at the thermal cycling temperatures, thus, no net TMF induced aging damage to the conductor materials.

Therefore, the aging issues associated with material creep, and fatigue and TMF can be ruled out for investigating the effective lifetime of SSC system operated at high temperature.

\subsection{General Formulation for SCC Lifetime Estimate}

The functionality of SSC components is illustrated in Figure 7-2, where the conductor section is responsible for transmitting the current and the core-grip section secures the steel-core. From thermal cycle simulation results, we observed that the conductor section acts as temperature driver that dictates the SSC system temperature, and the core-grip section is the main driver that secures the conductor cable at higher operating temperature (above $100^{\circ} \mathrm{C}$ ).

In general there are three main subjects that need to be addressed while investigating the effective lifetime of a SSC system, namely, geometry constraint integrity, cable tension effect and SSC system materials degradation. From thermal mechanical testing we have ruled out the influence of the material degradation in associated with the creep or fatigue for a high temperature operation. Moreover, from the degradation rate point, the impact of losing geometry constraint (such as void density increase and the decrease of compressive residual stresses) 
seems to be much more severe to the SSC effective lifetime than the impact of aluminum oxide build up with regard to the SSC system temperature increase. Therefore, in the SSC effective lifetime investigation we will focus on the subjects of tension effect and geometry constraint degradation.

In order to put FEM thermal cycle simulation results into a practical use to predict the SSC lifetime, we need to build a relationship between the time-dependent SSC-splice surface temperature and the associated effective constraint strength remaining in the SSC system to secure the conductor cable. The SSC's splice surface temperature can be easily monitored with a remote sensor; where the splice surface temperature can provide a direct link to the operationtime and operation-temperature history.

\subsection{Calculation of the Shear Resistance Force in SSCs}

In order to relate the SSC compressive stress to the effective shear resistance force against pullout of the conductor cable during the service, the temperature dependent friction coefficient needs to be considered. According to Refs 1-2, the friction coefficients of the steel and aluminum (Al) for the core-grip section are listed in Table 2.

Table 2. Temperature dependent friction coefficients of steel \& Al.

\begin{tabular}{|c|c|c|c|c|c|c|c|c|}
\hline Temperature $\left({ }^{\circ} \mathrm{C}\right)$ & 22 & 35 & 93 & 148 & 211 & 260 & 315 & 371 \\
\hline Friction Coefficient, $\mu$ & 0.610 & 0.545 & 0.259 & 0.115 & 0.064 & 0.047 & 0.035 & 0.020 \\
\hline
\end{tabular}

The room temperature static friction coefficient of $\mathrm{Al} \& \mathrm{Al}$ of 1.0 was chosen for a crimped SSC. [2] In general the friction coefficient varies linearly with tensile strength. [3] The trend of yield stress for 1350-H19 aluminum was used for estimating the friction coefficients of $\mathrm{Al} \& \mathrm{Al}$ for the conduction section. The estimated friction coefficient is listed in Table 3.

Table 3. Temperature dependent friction coefficients of Al \& Al.

\begin{tabular}{|c|c|c|c|c|c|c|c|c|}
\hline Temperature $\left({ }^{\circ} \mathrm{C}\right)$ & 25 & 100 & 150 & 177 & 205 & 230 & 260 & 315 \\
\hline Friction Coefficient, $\mu$ & 1.000 & 0.875 & 0.750 & 0.625 & 0.542 & 0.333 & 0.167 & 0.120 \\
\hline
\end{tabular}

Due to significant annealing of SSC aluminum components at high temperatures, shown in Fig. 8 , the friction coefficients at ambient temperature (or at cool-down) after the thermal cycling need to be modified (according to the tensile yield reduction ratio). At above $250^{\circ} \mathrm{C}$ the tensile yield stress of SSC aluminums starts to decrease significant, the Al vs. Al friction coefficient correction was based on the ratio of thermal cycling aging yield stress to that of baseline tensile yield stress, at ambient temperature. Regarding the friction coefficient of steel vs. Al, a similar approach was used to estimate the ambient temperature friction coefficient but with less reduction ratio. This is because that steel strength largely remains intact at the temperatures of interest, furthermore, a very rough interface exists between the core-grip and the steel-core. The estimated ambient-temperature friction coefficients at high temperature thermal cycle ranges are provided in Table 4 and Table 5 , for steel vs. $\mathrm{Al}$ and $\mathrm{Al}$ vs. Al, respectively. 
Table 4 Ambient temperature friction coefficients of steel \& Al.

\begin{tabular}{|c|c|c|c|c|c|c|c|}
\hline Aging Temperature $\left({ }^{\circ} \mathrm{C}\right)$ & 20 & 175 & 200 & 225 & 250 & 275 & 350 \\
\hline Friction Coefficient, $\mu$ & 0.610 & 0.610 & 0.580 & 0.549 & 0.427 & 0.366 & 0.366 \\
\hline
\end{tabular}

Table 5. Ambient temperature friction coefficients of Al \& Al.

\begin{tabular}{|c|c|c|c|c|c|c|c|}
\hline Aging Temperature $\left({ }^{\circ} \mathrm{C}\right)$ & 25 & 175 & 200 & 225 & 260 & 315 & 350 \\
\hline Friction Coefficient, $\mu$ & 1.000 & 0.950 & 0.860 & 0.630 & 0.180 & 0.160 & 0.16 \\
\hline
\end{tabular}

The shear resistance forces, for the full tension loading case, were evaluated at heat up and cooldown cycles for core-grip and conductor sections. The calculated shear resistance forces and the associated fitting equations are shown in Figure 8-1. Figure 8-1 clearly indicates the temperature dependence of SSC shear resistance capacity with the shear resistance constraint decreasing exponentially with temperature increase.

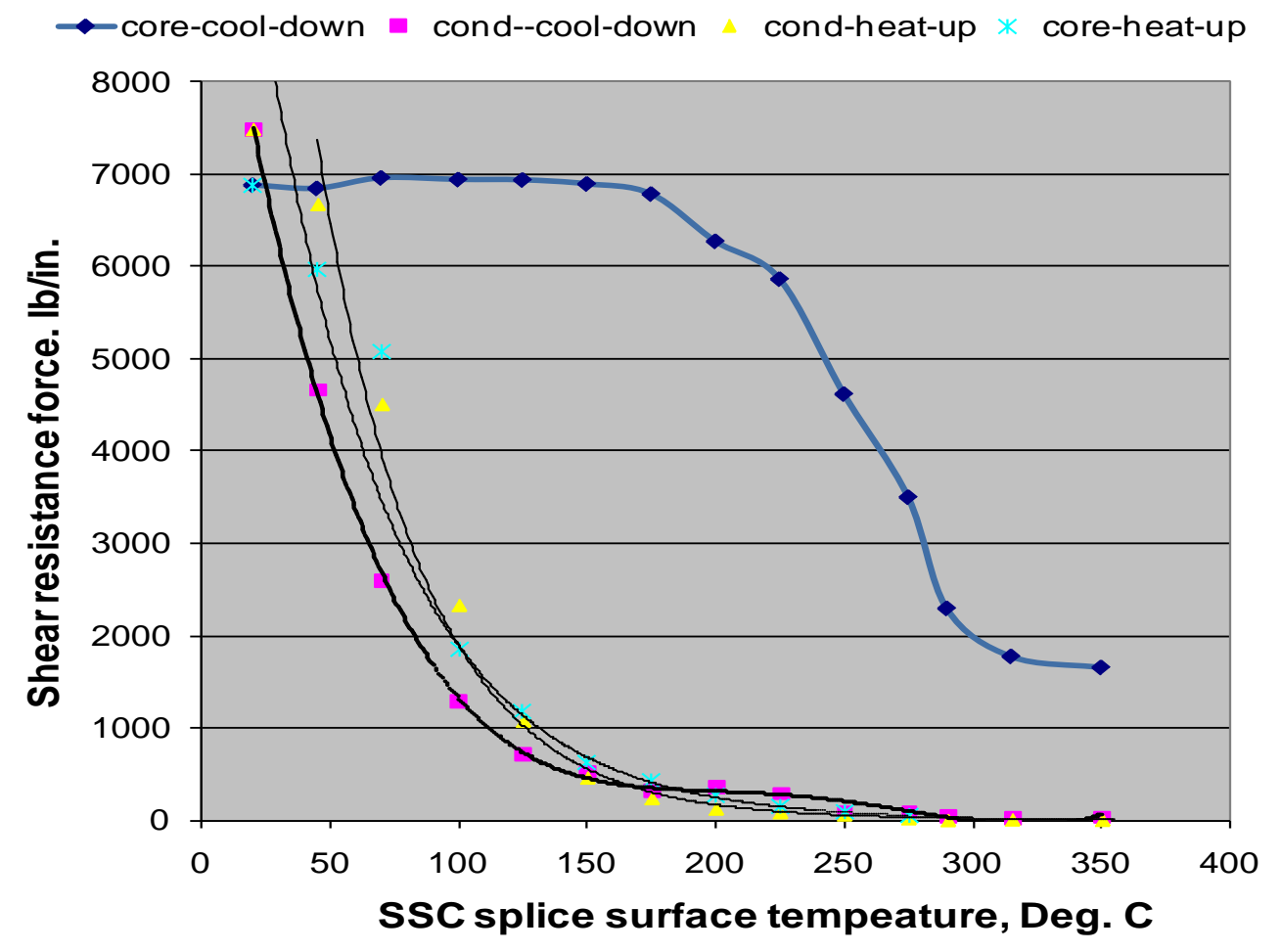

Figure 8-1 The temperature-dependent shear resistance trends for SSC Drake conductor system.

The shear resistance force calculated from "quench plus full-tension" case appears to be very close to that of full-tension case. There are some discrepancies between full-tension and quench plus full-tension cases from the core-grip compressive force data at cool-down cycle with temperatures above $250^{\circ} \mathrm{C}$, as shown in Figure 7-24. However, due to diminished friction coefficient of steel and $\mathrm{Al}$ at temperature above $250^{\circ} \mathrm{C}$, the difference of the resultant shear resistance forces between full tension and quench plus full tension cases is very small. Thus, 
only the full tension simulation data was used to develop the effective lifetime prediction models for SSC system. The summation of shear resistance forces from core-grip and conductor sections for heat-up and cool-down cycle are shown in Figure 8-2. From safety point of view, the shear resistance at heat-up cycle is the controlling factor and should be used as design criteria for lifetime estimate.

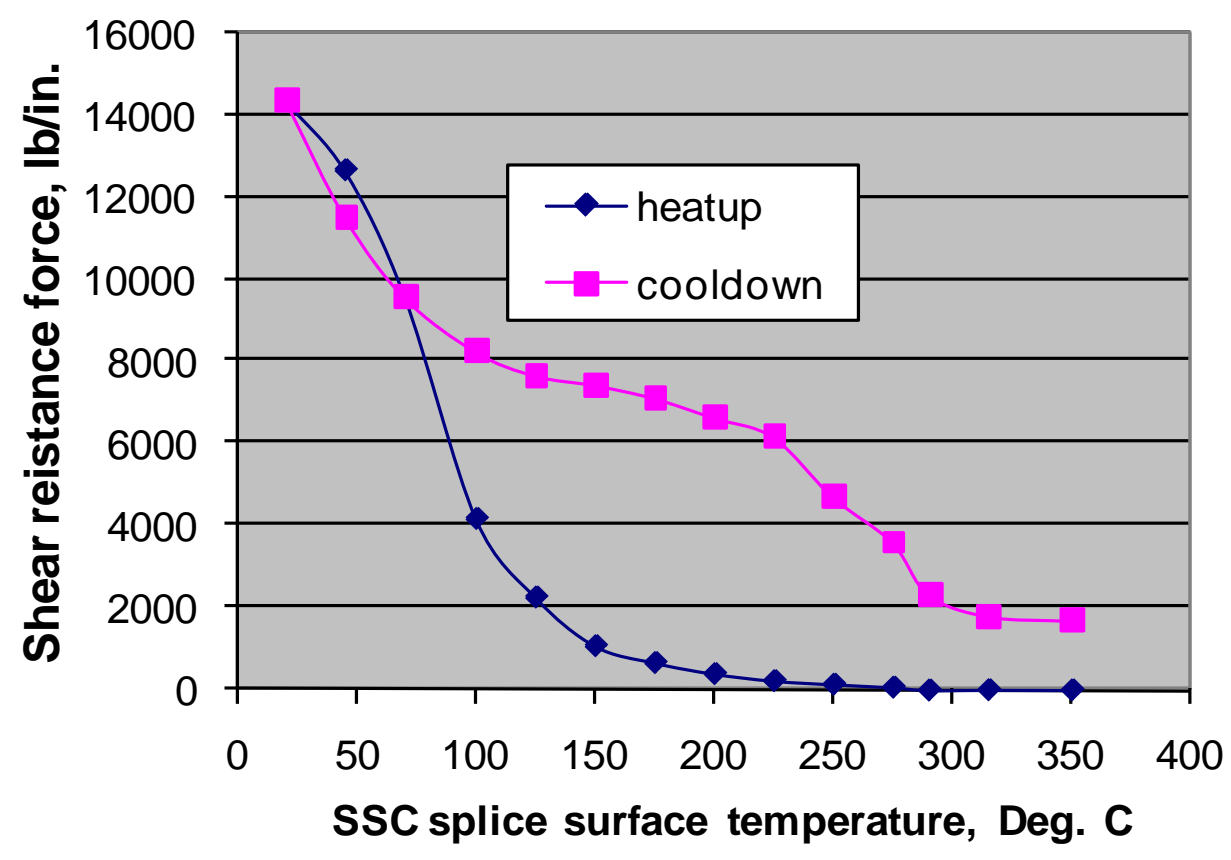

Figure 8-2 Sum of the shear resistance forces from the SSC conductor and core-grip sections.

\subsubsection{Confirmatory structure verification}

The ambient-temperature pull-out tests from the post-thermal cycling Drake SSC fittings were carried out, the test results are shown in Figure 8-3. Figure 8-3 also shows the shear resistance capacity of a ACSR Drake SSC splice with 16-in net length of conductor and core-grip sections. The details of the pull-out test are shown in Figure 8-4 and Figure 8-5.

With regard to the craftsmanship of forming SSC fitting and other uncertainties involved in the thermal cycling testing, the predicted fitting shear resistance capacity values of cool-down cycles fit remarkably well to that of the pull-out test results. The test results of the confirmatory structural verification experiments further validate the developed thermal cycling simulation protocols. 


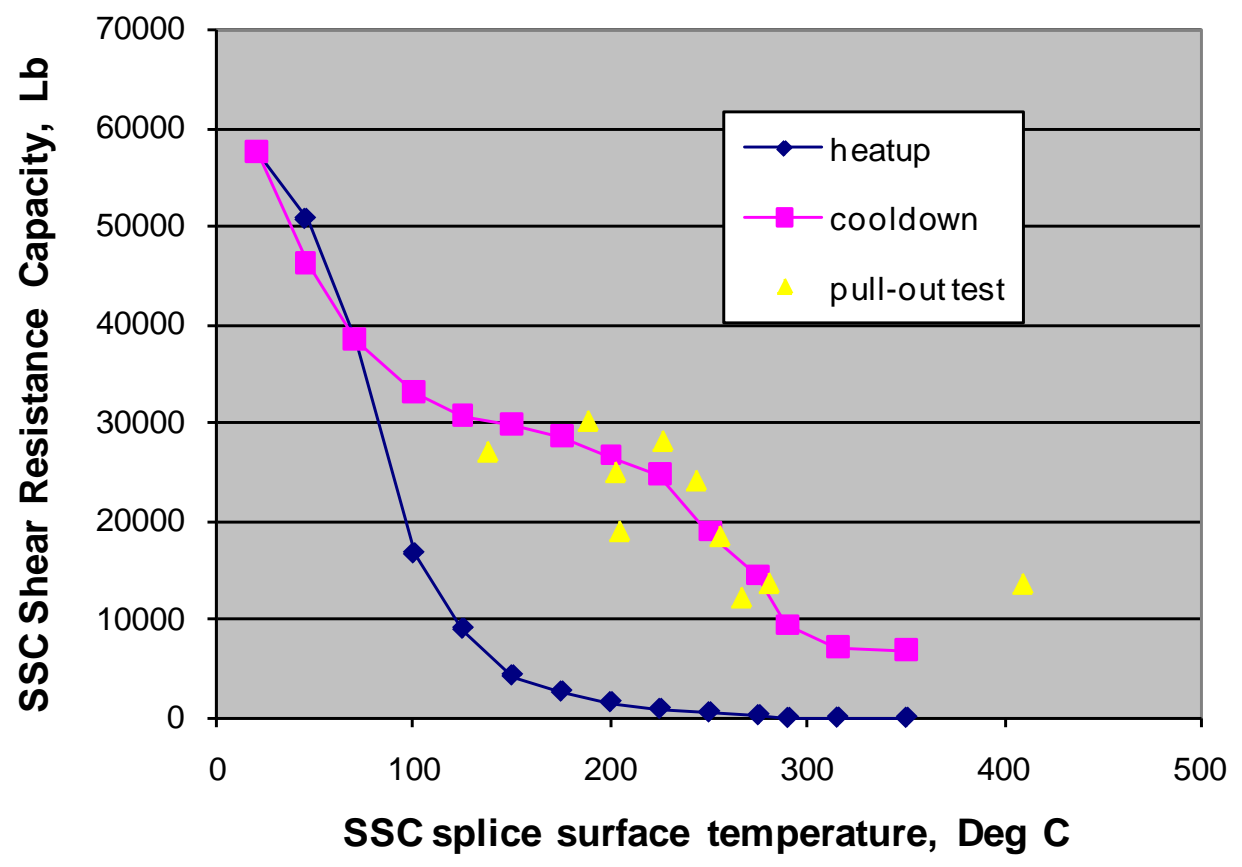

Figure 8-3 The shear resistance capacity of ACSR Drake SSC system with 16-in long net core-grip and conductor section.

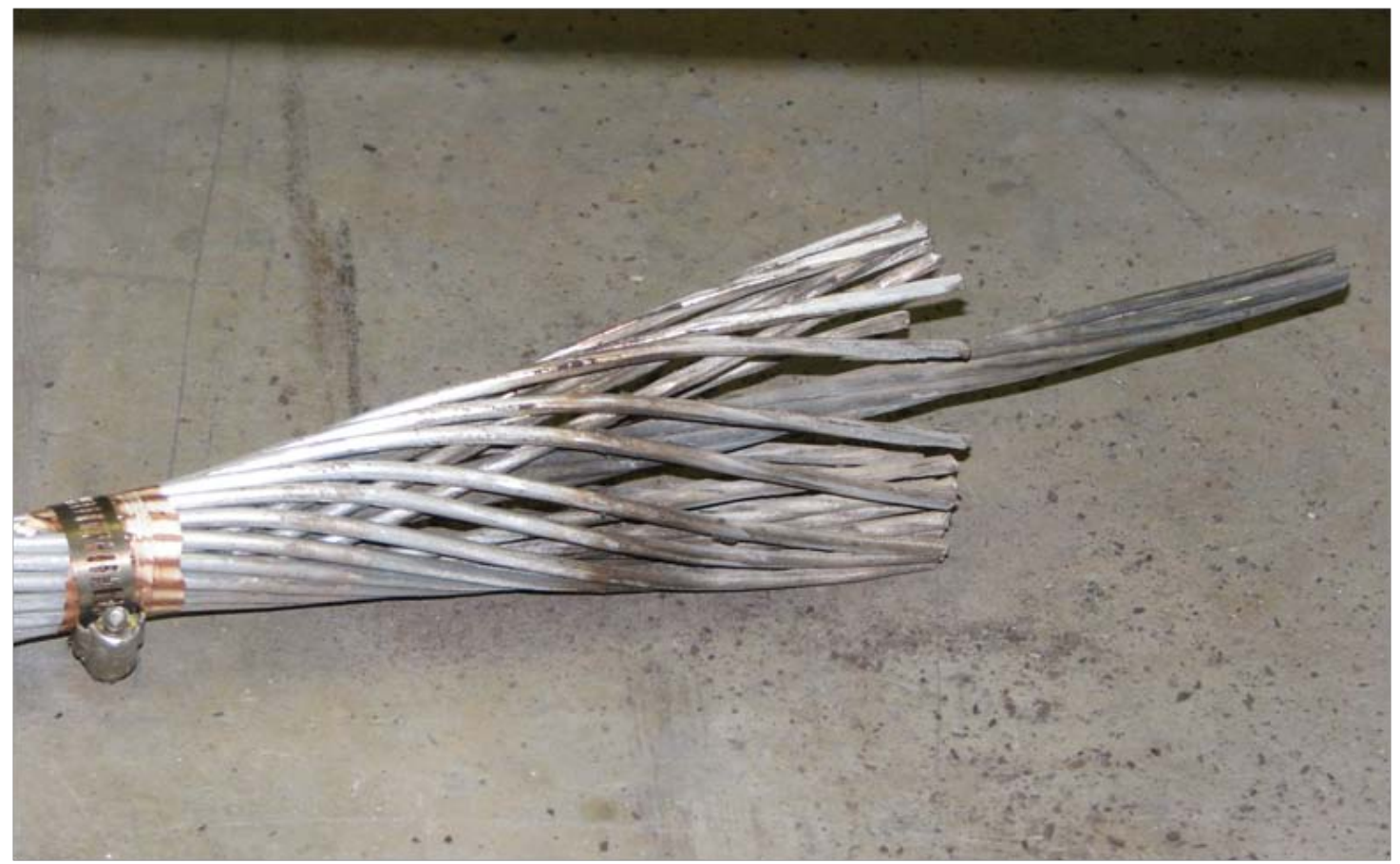

Figure 8-4 Drake conductor after SSC fitting pull-out test. 


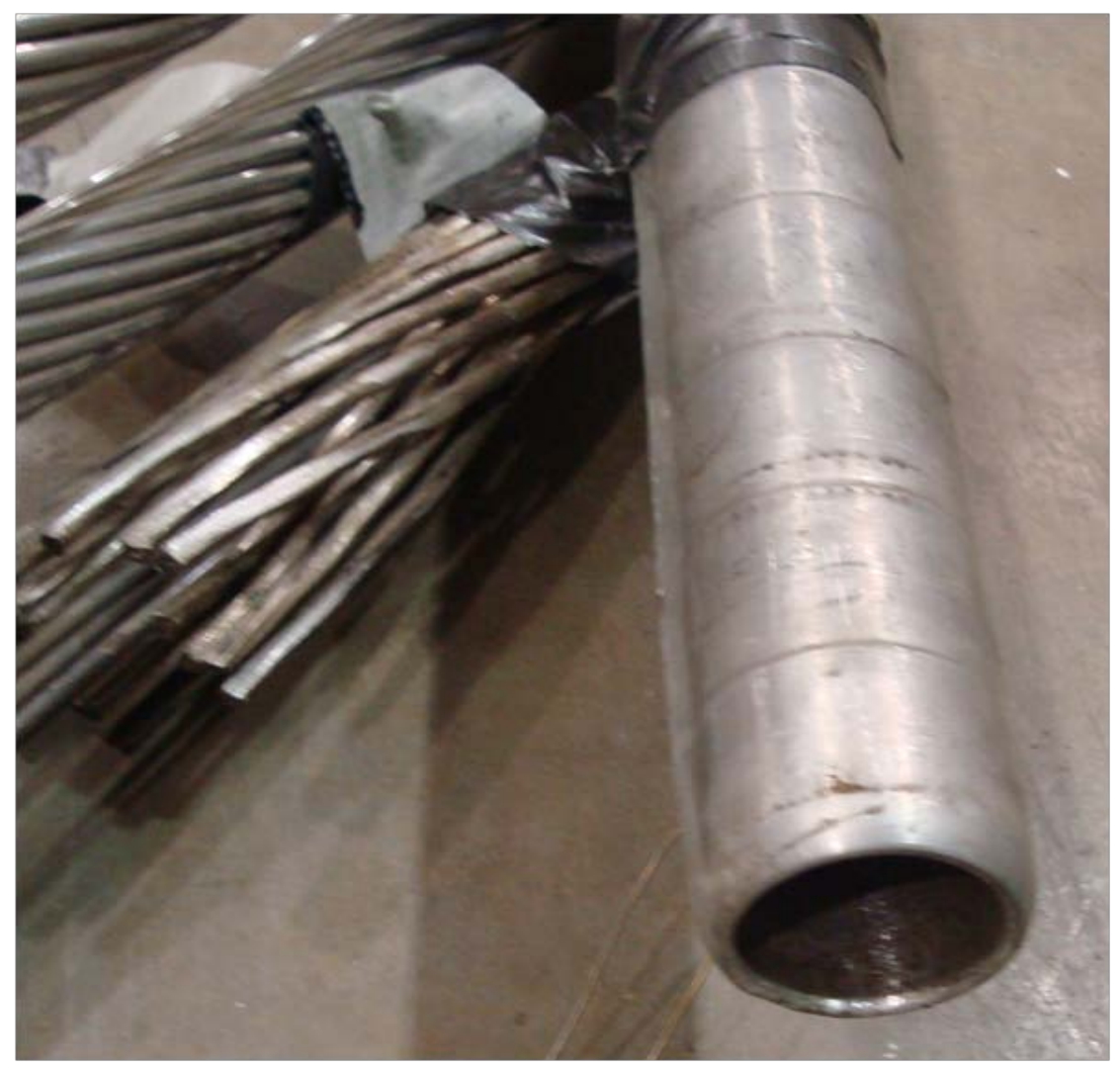

Figure 8-5 SSC fitting sleeve and the Drake conductor after the pull-out test.

\subsection{SSC Effective Lifetime Predictions}

\subsubsection{Governing equation of SSC shear resistance capacity}

The sole function of a SSC system is to secure the conductor cable to carry out the power transmission. Therefore, SSC must have sufficient shear resistance capacity to retain the aluminum conductor cable at any operation temperatures and loading conditions. The governing equation of the SSC lifetime prediction, for operation temperature $\geq 45^{\circ} \mathrm{C}$, can be written as below.

For heat-up cycle:

$$
R_{h}=F_{\text {cond }}(T, \text { heat-up }) * L_{\text {cond }}+F_{\text {core }}(T, \text { heat-up }) * L_{\text {core }}
$$

For cool-down (RT) cycle:

$$
R_{c}=F_{\text {cond }}(T, R T) * L_{\text {cond }}+F_{\text {core }}(T, R T) * L_{\text {core }}
$$

The effective SSC shear resistance capacity can be written as:

$$
\begin{aligned}
R_{S R} & =\operatorname{minimum}\left(R_{h}, R_{C}\right)=R_{h} \\
& =20992 * e^{-0.024 T} * L_{\text {cond }}+14449 * e^{-0.02 T} * L_{\text {core }}
\end{aligned}
$$


where, $\boldsymbol{F}$ is the governing equation of temperature-dependent shear resistance force, as stated in Figure 8-6, and the subscripts "cond" and "core" stand for conductor and core-grip sections, respectively. $T$ is the max. cycle temperature at SSC splice surface, and $L$ is the effective length. $\boldsymbol{R}_{\boldsymbol{h}}$ and $\boldsymbol{R}_{\boldsymbol{c}}$ are the shear resistance capacities at heat-up and cool-down cycles, respectively. $\boldsymbol{R}_{S \boldsymbol{R}}$ is the effective shear resistance capacity provided by SSC system.

For a safe operation, $\boldsymbol{F}_{S R}$ must greater than the tensile loading provided by the conductor system and its associated environment at any temperatures. Thus, the end of life of an SSC system can be defined as when the tensile loading of a power transmission line is approaching to the shear resistance capacity of the SSC system. From the design point, the SSC shear resistance capacity is dictated to the effective length of the core-grip and conductor sections. Thus, to ensure a safe operation of power transmission line at a target service temperature range with the designated cable loading, the designed SSC system must have sufficient length of the core-grip and conductor sections to provide enough safety margins.

A 600-ft span Drake conductor system, under heavy loading conditions stated in Table 2-4 of reference 4, was used to demonstrate the general applicability of this new method for investigating the SSC effective lifetime. For conservative illustration, the initial tension loading listed in Table 2-4 of reference 4 was used in demonstration. No tension loading was available from Table 2-4 for cable temperature greater that $100^{\circ} \mathrm{C}$, thus, the Catenary cable theory was used to estimate the conductor tensile loading at higher temperature; the calculated tension loading was then normalized with the reference 4's tension loading at $100^{\circ} \mathrm{C}$. A series of temperature-dependent SSC shear resistance capacities were generated for SSC systems with full lengths of 12-in, 16-in, 20-in, 24-in, and 40-in, respectively, with the assumption of equal length for the conductor and the core-grip sections, as shown in

Figure 8 -6. Which indicates that all the SSC systems, with the initial 25\% RBS tension loading, have sufficiently high shear resistance capacity to secure the conductor cable at SSC splice surface temperature below $100^{\circ} \mathrm{C}$. Figure 8-7 shows the detailed view of Figure 8-6 for shear resistance capacity less than 5,000lb. Figure 8-7 shows that for a 12-inch long SSC splice system, formed by a ALCOA 60 ton 12CD die, failure (or separation) will likely occur at above $150^{\circ} \mathrm{C}$ (splice surface temperature), and for a 40-inch long SSC splice it may extend the SSC system service life to $225^{\circ} \mathrm{C}$. From this exercise, it clearly shows that the current SSC system will function well for SSC splice surface temperature below $100^{\circ} \mathrm{C}$ or up to $125^{\circ} \mathrm{C} 150^{\circ} \mathrm{C}$ for emergency situation; and the shear resistance capacity of the current SSC system apparently is not an efficient design for high temperature operation. 


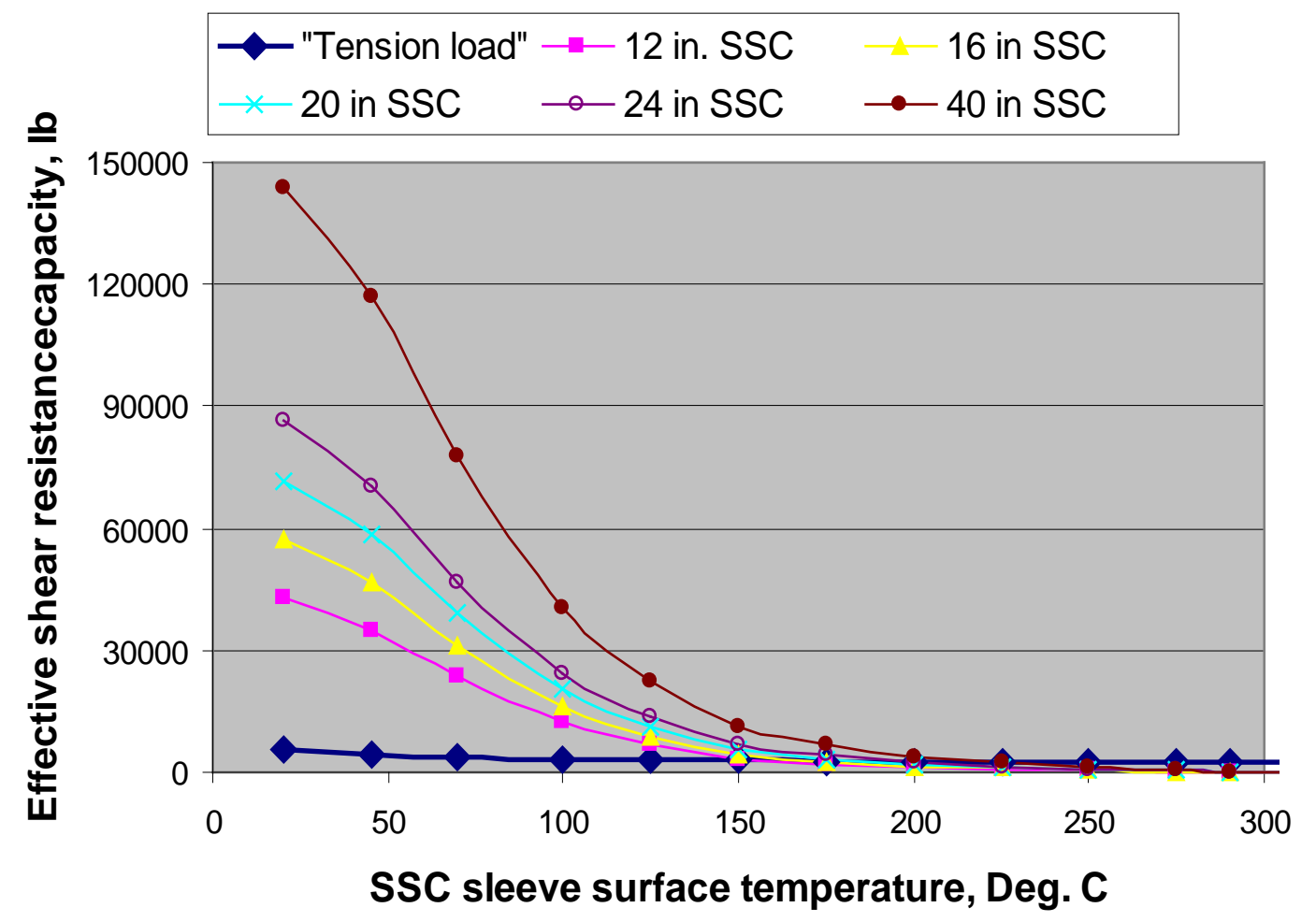

Figure 8-6 The shear resistance capacity for Drake SSC systems.

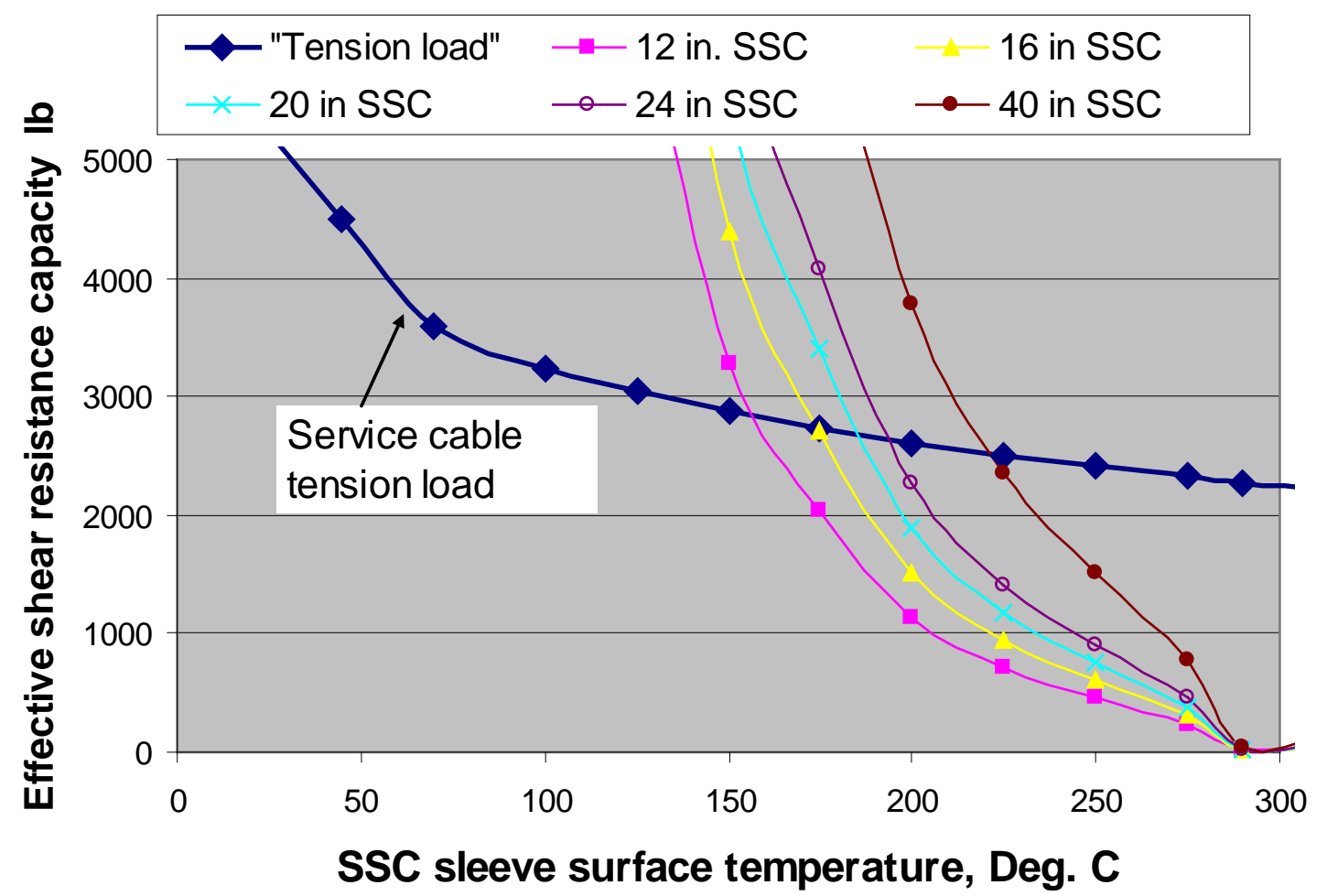

Figure 8-7 Drake SSC shear resistance capacity at high temperature. 


\subsubsection{Governing equation of SSC surface temperature history}

Time-frequency-dependent SSC surface temperatures were observed during thermal cycle experiments, where the ACSR Drake conductor operation temperature was controlled at $150^{\circ} \mathrm{C}$, except between 97 to 129 cycles the conductor temperature was controlled at $90^{\circ} \mathrm{C}$. Some representative SSC surface temperatures are shown in Figure 8-8, and this frequencydependent temperature history data was used to develop the governing equation that relates the SSC surface temperature to the thermal cycle frequency (or time history).

Due to significant tension drops during the SSC thermal cycling experiments, the tested spans were re-tensioned at the following cycle numbers: 120, 407, 611, 858, 974, or whenever the aging SSC was replaced with a new SSC fitting. The tension history data of the 4 SSC tested spans are shown in Figure 8-9, where the conductor tension load decreases continuously during thermal cycling. This is the direct indication of conductor slippage in an aging SSC system. Re-tension of SSC spans back to 20\% RBS ( 6200 lb) will introduce relatively large slippage jumps, and through slippage it will produce some fresh (good) contact surfaces at SSC system interfaces. This will reduce the resistivity of aging SSC; thus, the decrease of SSC surface temperature. However, the increase of SSC surface temperatures will resume right after restarting thermal cycling, as shown in Figure 8-8.

Large temperature data scatter, inherited from both SSC forming quality and thermal cycling experiments, seems to also depend on the test span and SSC sequential position within the test span (normally one test span contains 5 SSC splices including two SSC dead end splices). The re-tension and the replacement of SSC fittings during the thermal cycle testing, (for example, the SSC surface temperature of TC55 rose quickly in the initial testing phase and it was replaced after 43 thermal cycles, shown in Figure 8-8), may further complicate the effort in developing trend curves of frequency-dependent SSC surface temperatures.

Nevertheless, SSC surface- temperature history data shows that the SSC aged quickly within the first 100 cycles and then approached steady state temperature; where steady-state surface temperatures of normal operating SSC is around $160^{\circ} \mathrm{C}$ to $200^{\circ} \mathrm{C}$, and around $160^{\circ} \mathrm{C}$ to $220^{\circ} \mathrm{C}$ for some quenched data. In order to extend the trend curve to a wide spectrum of conductor operating temperatures, one assumption was made - the general trend of the governing equation, based on $150^{\circ} \mathrm{C}$ conductor operating temperature, remains the same and varies linearly with other operation temperatures. According to this methodology, a frame work of SSC surface temperature-frequency history charts, which is a function of conductor operation temperatures, were developed. It is noted that the change of conductor controltemperature from $150^{\circ} \mathrm{C}$ to $90^{\circ} \mathrm{C}$ resulted in a $40^{\circ}$ to $60^{\circ} \mathrm{C}$ decrease in SSC surface temperature as observed from Figure 8-8.

Based on the above and the temperature data shown in Figure 8-10, which contains data up to first re-tension (less than 400 cycles) and excluding data associated with $90^{\circ} \mathrm{C}$ conductor control temperature period, a governing equation of frequency dependency SSC surface temperature, $T$, was developed as described below.

$$
T=0.6375 * T_{\text {cond }} * N_{f}^{0.0969}
$$

where, $\boldsymbol{T}_{\text {cond }}$ is the control conductor temperature and $\boldsymbol{N}_{\boldsymbol{f}}$ is the number of thermal cycles. 

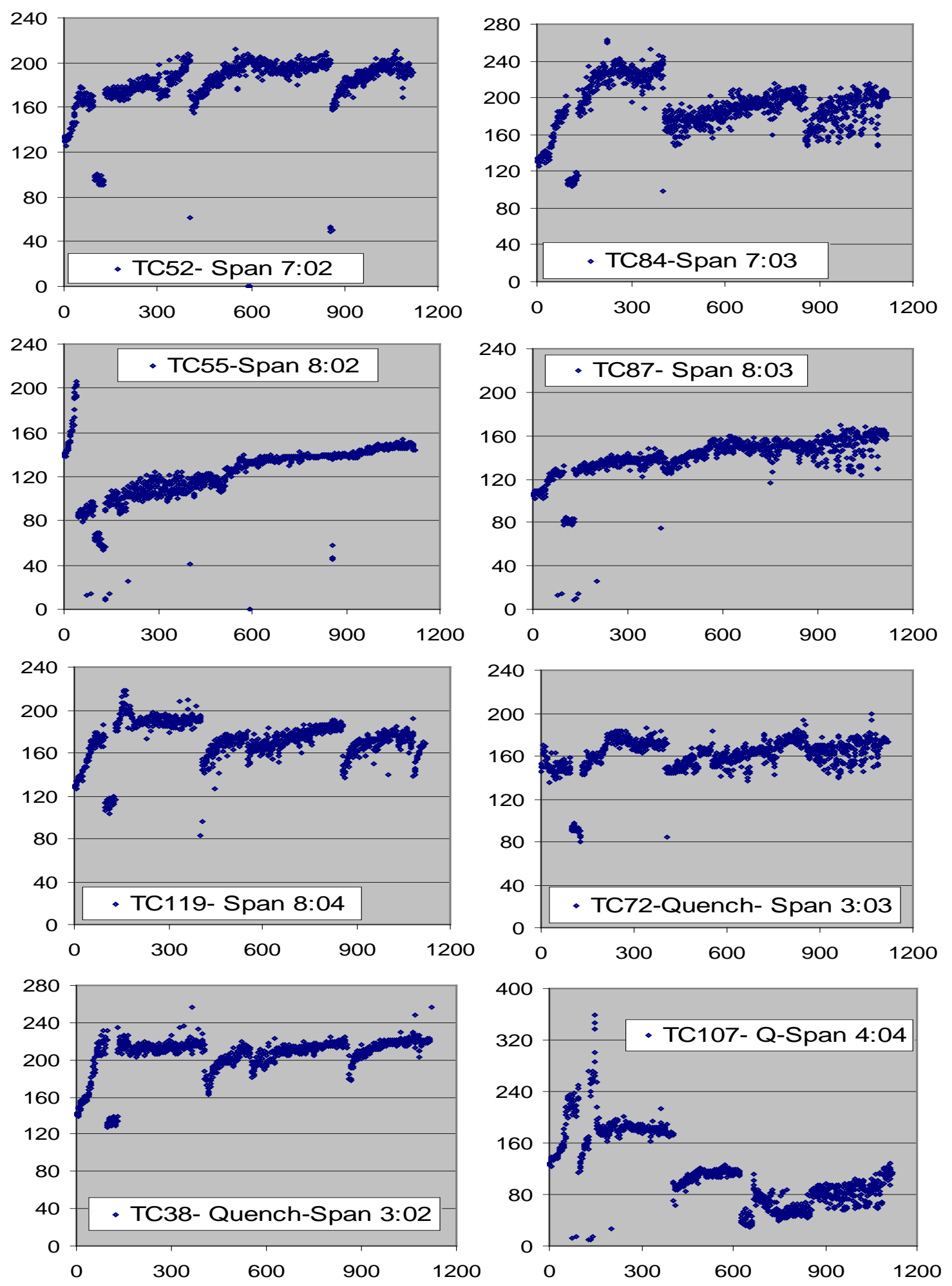

Thermal cycle number

Figure 8-8 Maximum cycle temperature $\left({ }^{\circ} \mathrm{C}\right)$ at SSC splice surface. 


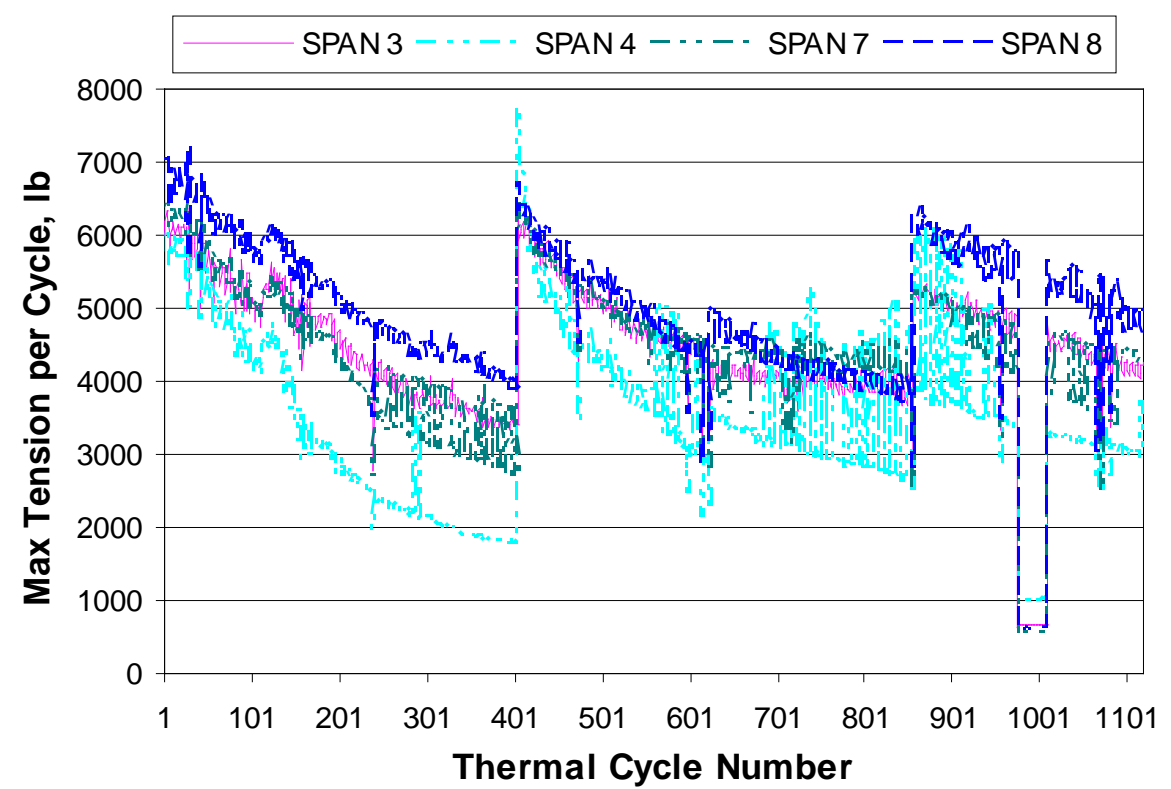

Figure 8-9 The tested SSC spans tension-thermal cycle history; spans 3 and 4 were designated for water quenched test spans.

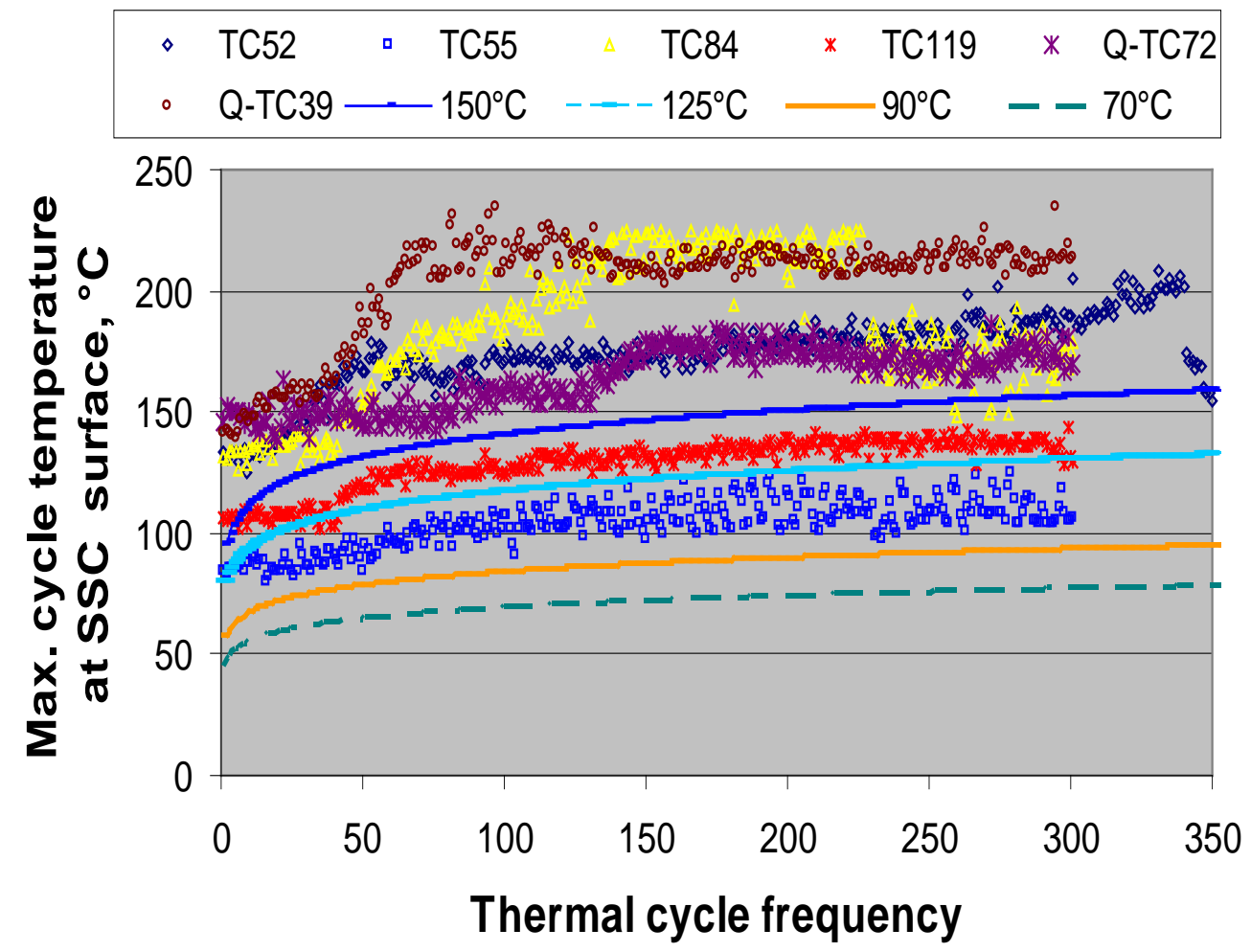

Figure 8-10 SSC surface temperature history obtained from thermal cycle experiments with $150^{\circ} \mathrm{C}$ conductor control temperature. 
The SSC surface temperature trend curves for conductor cable temperatures with $150^{\circ} \mathrm{C}, 125^{\circ} \mathrm{C}$, $90^{\circ} \mathrm{C}$, and $70^{\circ} \mathrm{C}$ are also illustrated in Figure 8-10. By combined Eq. 1 and Eq. 2 the overall governing equation for the SSC effective lifetime can be written as:

$$
\begin{aligned}
& R_{S R}=20992 * e^{-0.024 T} * L_{\text {cond }}+14449 * e^{-0.02 T} * L_{\text {core }} \\
& =20992 * \exp \left(-0.024 * 0.6375 * T_{\text {cond }} * N_{f}^{0.0969}\right) * L_{\text {cond }}+ \\
& 14449 * \exp \left(-0.02 * 0.6375 * T_{\text {cond }} * N_{f}^{0.0969}\right) * * L_{\text {core }}
\end{aligned}
$$

\subsubsection{Demonstration of SSC effective lifetime prediction}

A half section of an ACSR Drake conductor SSC fitting has 4.5-in long core-grip section and about 4.5-in long conductor section, with the consideration of the taper sleeve ends (shown in Figure 5-24) and the conservative point, the net length for core-grip section and the conductor section are estimated at 4-in each. The associated SSC effective lifetime trends for conductor operating temperatures ranges from $70^{\circ} \mathrm{C}$ to $300^{\circ} \mathrm{C}$, are illustrated in Figure 8-11. The details of SSC shear capacity below 6000-lb are shown in Figure 8-12.

\subsubsection{Using SSC shear resistance capacity for SSC lifetime estimate}

The application of the developed protocol for effective lifetime estimate is illustrated in the following step-by-step example.

(1) Estimate the SSC system effective lifetime of a 600-ft-span Drake conductor system under hea vy loading at $125^{\circ} \mathrm{C}$ and $150^{\circ} \mathrm{C}$ conductor operating temperature.

Solution:

a) The service conductor cable tension loads for a 600-ft span Drake conductor system, under heavy loading conditions, are shown in Figure 8-7.

b) From Figure 8-7, for conductor operated at $125^{\circ} \mathrm{C}$, the required service tension load is 3040-lb.

c) Based on this screen criterion and from Figure 8-12, for a conductor operated at $125^{\circ} \mathrm{C}$ the SSC system can meet the screening criterion of 3040-lb tension-load up to 3100 cycles.

d) While for a $150^{\circ} \mathrm{C}$ conductor operating temperature, the screen criterion is 2873-lb tension load.

e) At about 550 cycles the SSC shear resistance capacity is about 2922-lb, shown in Figure 8-12, which is slightly larger than the required service tension load of 2873-lb, shown in Figure 8-7. 


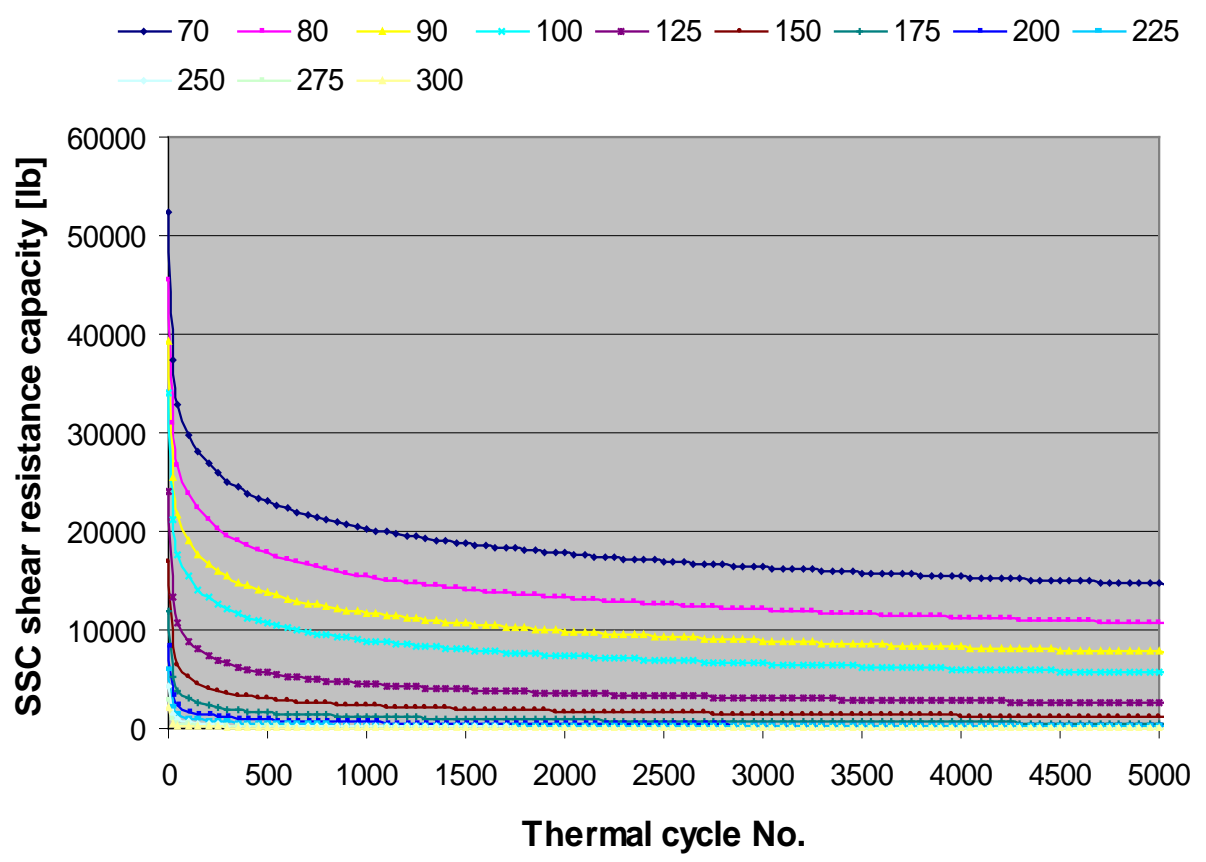

Figure 8-11 ASCR Drake SSC effective lifetime trend curves.

$\longrightarrow 100 \rightarrow-125 \longrightarrow 150 \multimap 175-200-225-250 \longrightarrow 275-300$

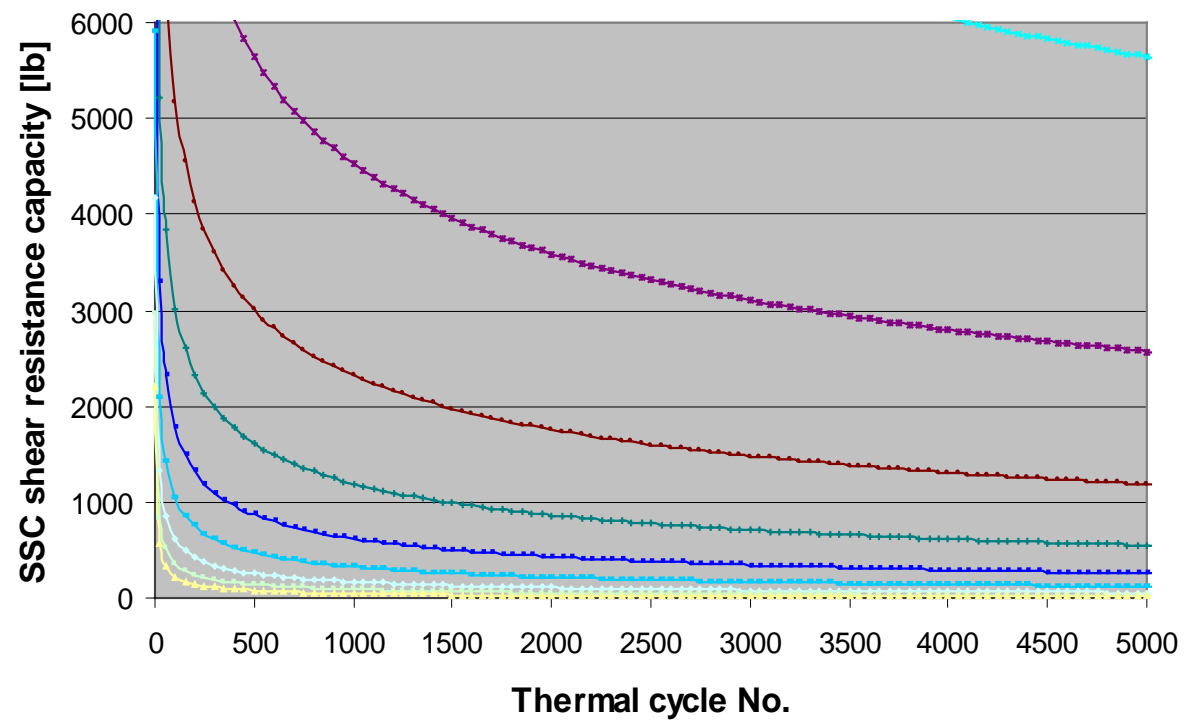

Figure 8-12 ACSR Drake SSC lifetime prediction for shear capacity $<6000$ lb.

Therefore, the allowable lifetime or cycling frequency for the 600-ft-span Drake conductor system operated at $125^{\circ} \mathrm{C}$ and $150^{\circ} \mathrm{C}$ are 3100 and 550 cycles, respectively. Assuming two cycles per day, the effective lifetime of SSC system will be 1550 and 275 days for $125^{\circ} \mathrm{C}$ 
and $150^{\circ} \mathrm{C}$ operating temperature, respectively. As regarding the $100^{\circ} \mathrm{C}$ conductor operating temperature, the service screening criterion is $3241 \mathrm{lb}$, based on equation 3 the estimated lifetime cycles is about 27,851 thermal cycles; as for a two-cycle per day, the equivalent lifetime is about 38 years.

(2) Estimate the accumulated damage of a Drake SSC system, with 600-ft-span of heavy loading, due to different operating temperatures, for example, the conductor operated at $125^{\circ} \mathrm{C}$ for 200 cycles and $135^{\circ} \mathrm{C}$ for 300 cycles.

Solution:

a) Substitute $125^{\circ} \mathrm{C}$ and 200 cycles into Eqn. 3, one obtains the SSC shear resistance capacity of 7510-lb, i.e., the remaining SSC strength after first 200 cycles.

b) Based on the 7510-lb remaining strength, the equivalent thermal-cycle at $135^{\circ} \mathrm{C}$ operating temperature is 94 .

c) Substitute $135^{\circ} \mathrm{C}$ and $(300+94)$ cycles into Eqn. 3, one obtains the SSC shear resistance capacity of 4827-lb.

d) The remaining SSC strength due to the accumulated damages is equal to 4827-lb.

e) The screening criteria at $135^{\circ} \mathrm{C}$ is 2973 -lb, the corresponding estimated effective life cycles is 1601 cycles. If conductor continuing operated at $135^{\circ} \mathrm{C}$, the remaining life cycles after the 300 cycles operated at $135^{\circ} \mathrm{C}$ will be equal to $1601-394=1207$ cycles. The remaining SSC lifetime (for a 2-cycle/day event) is equal to 1.65 years.

In accordance to AISC Specification [5] for friction-type connector, a safety factor of 1.76 of allowable stress design is recommended to be applied to SSC shear resistance capacity for the SSC effective lifetime estimate.

\subsection{REFERENCES}

[1] M. Awang, V. H. Mucino, Z. Feng, and S. A. David, "Thermo-Mechanical Modeling of Friction Stir Spot Welding Process," SAE 2005 World Congress \& Exhibition, April 2005.

[2] Coefficient of friction for aluminum/aluminum and aluminum/ steel:http://www.roymech.co.uk/Useful_Tables/Tribology/co_of_frict.htm

[3] Peterson, M.B., and Ramalingam, S., in "Fundamentals of Friction and Wear of Material," edited by D.A. Rigney, ASM Metals Park, OH, 1981, p.342.

[4] Ridely Thrash, et. al, Southwire Company Overhead Conductor Manual, First Edition, 1994.

[5] Specification for Structural Steel Building, American Institute of Steel, Construction, INC., March 9, 2005. 


\section{9 sUMMARY}

As part of a project sponsored by EPRI at Oak Ridge National Laboratory to develop life prediction models for High-Temperature Low-Sagging Conductors a protocol to provide accurate estimates of the lifetime of SSC was developed. Most of the work has been focused on splice-conductor interfaces because these have been identified as the weakest links in transmission lines. In particular, single-stage connectors have been analyzed because they experience greater accumulated damage than two-stage connectors. The key findings are:

- The baseline results of numerical simulations have shown that connector materials experience large plastic deformation during the crimping process, which is necessary to induce compressive residual stress in the connector system. The FEM model predictions were found to be in good agreement with experimental residual stress measurements obtained by neutron diffraction techniques. These results are important because they provide a point of reference for the formulation of life prediction models.

- The kinetics of thermo-mechanical processes, such as cyclic thermal fatigue and creep deformation of aluminum alloys were studied to provide the information necessary for the formulation of such models and for thermal-mechanical cycling simulation.

- The integrity of SSC systems under thermal cycling at high operating temperature has been analyzed through this research. The results of FEM simulations for SSC forming mechanism and SSC aging induced by thermal cycling were found to be in good agreement with experimental residual stress measurements obtained by neutron diffraction techniques and SSC aging/degradation trends obtained from the thermal cycling experiments of SSC Drake conductor systems. A finite element simulation protocol was also developed to provide guidance in effective lifetime estimate of SSC system performance. The main findings are

$\checkmark$ Compressive residual stress field and the associated shear resistance capacity within a SSC system can serve as good index for effective lifetime estimate.

$\checkmark$ The thermal cycling has significant detrimental effect on SSC lifetime, especially under high temperature operation.

$\checkmark$ Tension loading needs to be considered in SSC lifetime investigation, especially for consideration in combination with dynamic tension loading.

$\checkmark$ Both the compressive residual stresses at cool-down and at heat-up cycle temperatures need to be considered in evaluating the SSC system performance and lifetime.

$\checkmark$ It is expected that the developed protocol and the associated SSC life prediction models can be very beneficial to both power grid and power transmission line operators and the conductor system designer regarding safe operation to meet the current power demand. 
The schematic diagram of the developed SSC effective lifetime estimate protocol is illustrated in Figure 9-1. This protocol can be generally applied or extended to other HTLS connector systems.

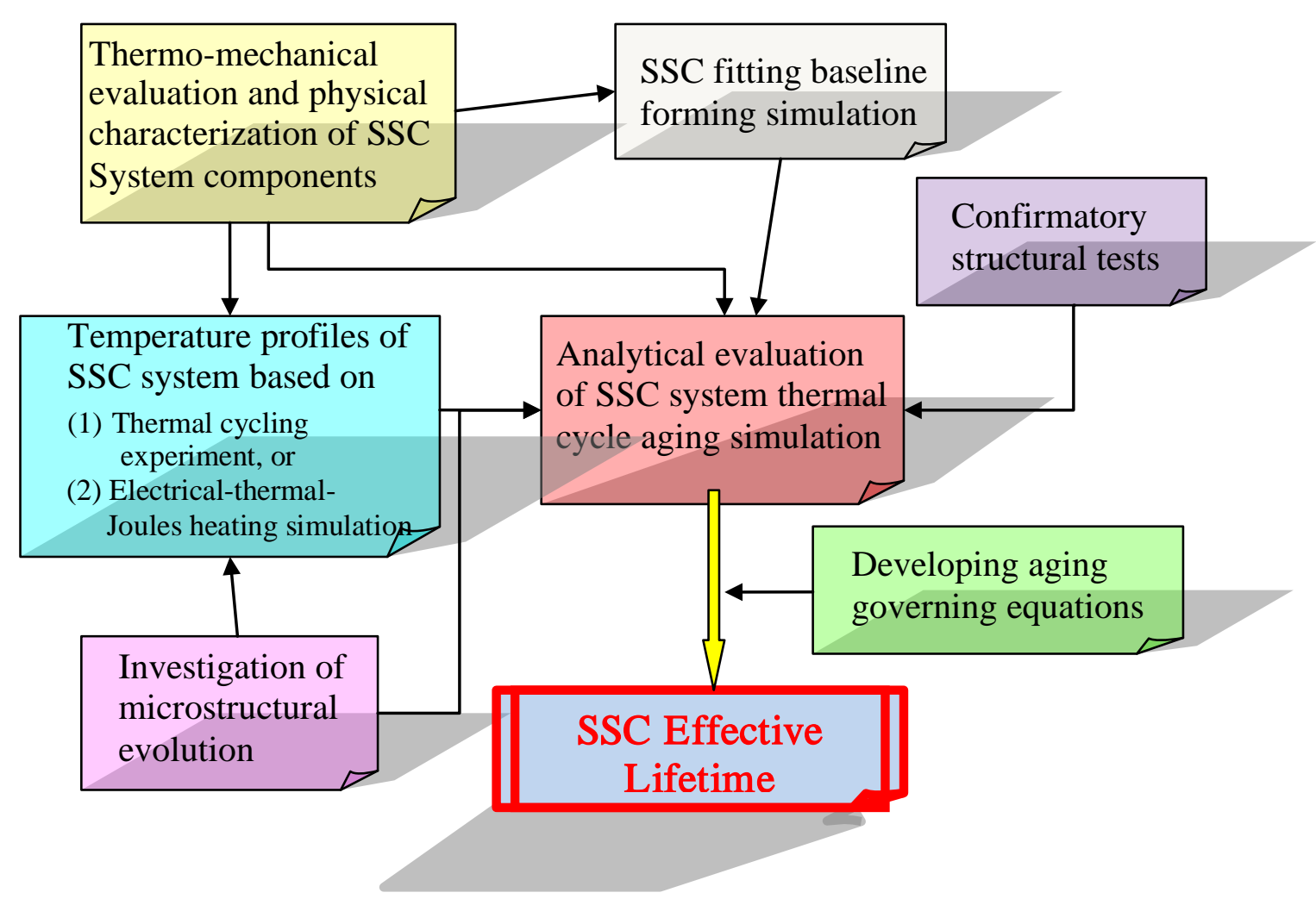

Figure 9-1 Schematic diagram of the developed SSC effective lifetime estimate protocol

In summary, to provide a realistic prediction for investigating the SSC system performance and the associated effective lifetime the following conditions/parameters must be taken into account:

(1) Inherited temperature-dependent tensile loading of the conductor system, including the environmental effects

(2) SSC system forming mechanism and the associated geometry constraint effect

(3) SSC system components thermal-mechanical behaviors and the temperature dependent physical properties

(4) Temperature profiles within the SSC system during thermal cycling, this can be obtained either from thermal cycling experiments, or an electrical-thermal-mechanical analytical simulation

(5) Benchmark the analytical simulation results with well-defined experimental measurements.

Generally, a true time-temperature-dependent analytical investigation of SSC system aging will require performing thermal-mechanical simulation analyses coupled with the electrical-joule 
heating evaluation, to properly evaluate and calibrate time-temperature dependent responses of the SSC system during power operation. However, to carry out meaningful electrical-thermalmechanical coupling analyses for a SSC system, it will require detailed information related to SSC system materials' microstructure evolution, such as temperature-time dependent oxide scale build-up and contact-interface degradation (regarding physical thermal conductance and electrical conductance properties), and the impact of the joint compound integrity to SSC system performances. Due to lack of such information and being out of the scope of this investigation, the electric joule heating evaluation coupled with the thermal-mechanical evaluation was not performed, instead the issue related to temperature profiles of a SSC system was directly referred to the experimental temperature profile results obtained from the SSC thermal cycling experiments. In view to developing practical and meaningful prediction models, the latter approach of using the experimental thermal profile results is preferred and it has less error prone than electrical-thermal simulation approach due to lack of microstructure evolution data. Nevertheless, after resolving the issue associated with the SSC system microstructure evolutions, developing integrated electrical-thermal-mechanical analyses protocol for the SSC or other splice connector system will be of interest and the next step in developing the robust time-temperaturedependent prediction models for investigating the SSC or other conductor splice connector system performance and their associated effective lifetime estimate.

This research addresses the needs of electric utilities because it provides them with tools for improving the reliability of the electrical grid through the formulation of time-dependent failure criteria that will assure adequate service life of ACSR SSC system. These tools will aid electric utilities in making decisions regarding maintenance of the grid and response to power demands.

The developed governing equation that can be used for predicting the SSC effective lifetime is described below.

$$
\begin{aligned}
& R_{S R}=20992 * \exp \left(-0.024 * 0.6375 * T_{\text {cond }} * N_{f}^{0.0969}\right) * L_{\text {cond }}+ \\
& 14449 * \exp \left(-0.02 * 0.6375 * T_{\text {cond }}{ }^{*} N_{f}^{0.0969}\right) * * L_{\text {core }}
\end{aligned}
$$





\section{ACKNOWLEDGEMENTS}

The authors would like to thank Ke An and Cam Hubbard for collaboration on the neutron residual stress measurements; Ken Liu for assistance in mechanical testing; Jeff Young and Tip Goodwin of EPRI HASLET for providing the temperature profile measurements of splice connectors; J. A Graziano and J. Chan for guidance and financial support. This project was funded jointly by EPRI HTLS Program and DOE Office of Electricity Delivery and Energy Reliability. 



\section{$A$ BENDING MOMENT EVALLATION FOR POWER TRANSMISSION LINES}

In general, three configurations exist in a conductor cable structure, namely,

(1) cable continues through the suspension ends, which can be characterized with a simple support fixed-ends with lateral load (cable weight) model, where the maximum bending moment is located at the suspension ends;

(2) cable stop at one suspension end and continue through the other suspension end, which can be characterized with a simple support, one-end fixed and one-end hinge model, where the maximum bending moment is at the continuing cable suspension end; and

(3) cable terminated at the suspension ends, which can be characterized as a simple support hinged-ends with lateral load model, where the maximum bending moment is the midspan.chp62cht

Within the three scenarios, based on the simple beam theory (first approximation), the greatest bending moment will occur at continuing suspension end of Scenario 2 and at the mid-span of Scenario 3. The detailed bending moment evaluations of the Scenarios 3 are further discussed below. Fig. 1 provides the geometry formulations of a cable with a parabolic geometry, as described "Structural Steel Designer's Handbook." Three approaches were used to carryout the cable bending moment investigation, namely, simplified beam theory, detailed tie-rod theory, and cable curvature theory approaches. The ACSR Drake conductor was used in the cable bending moment investigation; the associated properties are described below.

\section{Data from Southwire Overhead Conductor Manual}

For a ACSR Drake Conductor: Kcmil=795, Al/St=26/7

$\mathrm{Al}$ (area) $=0.6247 \mathrm{in}^{2}$, Total area $=0.7264 \mathrm{in}^{2}, \mathrm{St}($ area $)=0.101 \mathrm{in}^{2}$

Unit length cable weight $\mathrm{w}_{0}=1.094 \mathrm{lb} / \mathrm{ft}$.

The moment of inertia of steel core is $0.000923 \mathrm{in}^{4}$ and the largest boundary radius (y-axis) is $0.2040 \mathrm{inch}$; the moment inertia for the aluminum conductors is $0.0532 \mathrm{in}^{4}$, and the largest boundary radius is 0.5538 inch.

The overall rigidity EI is equal to

$$
\mathrm{EI}_{\mathrm{AS}}=\mathrm{E}_{\mathrm{ST}} * \mathrm{I}_{\mathrm{ST}}+\mathrm{E}_{\mathrm{AL}} * \mathrm{I}_{\mathrm{AL}}=(30 \mathrm{E}+6 \mathrm{psi} * 0.000923)+(10 \mathrm{E}+6 \mathrm{psi} * 0.0532)=\underline{5.6 \mathrm{E}+05 \mathrm{lb}-\mathrm{in}^{2}}
$$

The effective of the moment inertia (based on aluminum material) is equal to

$$
\mathrm{I}_{\text {eff }}=\left(\mathrm{E}_{\mathrm{ST}} / \mathrm{E}_{\mathrm{Al}}\right) \mathrm{I}_{\mathrm{ST}}+\mathrm{I}_{\mathrm{Al}}=3 * 0.000923+0.0532=0.05597 \mathrm{in}^{4}
$$



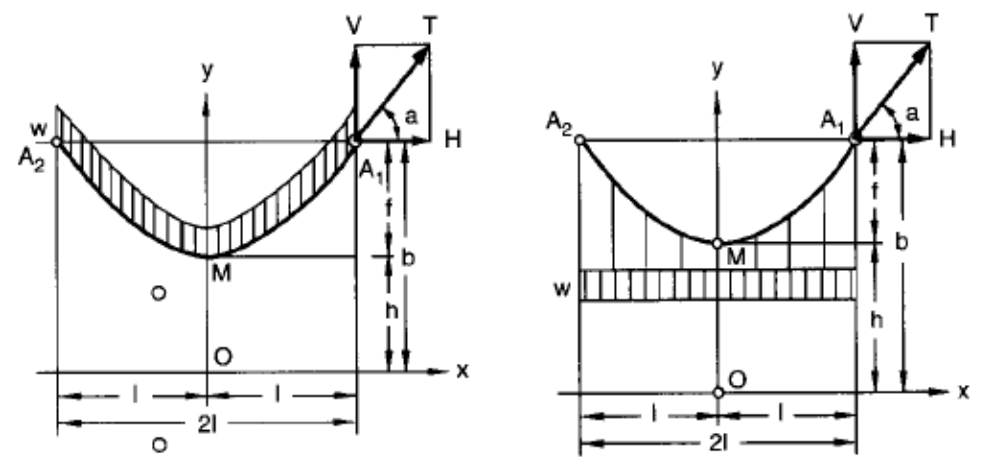

\begin{tabular}{|c|c|c|}
\hline & Catenary & Parabola \\
\hline Cable ordinate $y_{\dagger}^{\dagger}$ & $\begin{aligned} y & =\left(e^{x / h}+e^{-x / h}\right) h / 2 \\
& =h \cosh x / h\end{aligned}$ & $y=h+x^{2} / 2 h$ \\
\hline $\begin{array}{l}\text { Coordinate } b \text { of } \\
\text { attachment points } A_{1} \\
\text { and } A_{2}\end{array}$ & $\begin{aligned} b & =f+h \\
& =h \cosh l / h\end{aligned}$ & $\begin{aligned} b & =f+h \\
& =h+l^{2} / 2 h \\
& =\left(l^{2}+4 f^{2}\right) / 2 f\end{aligned}$ \\
\hline $\begin{array}{l}\text { Sag-span ratio } a \\
\text { Slope } y^{\prime} \text { of cable } \\
\text { Ordinate } h \text { of cable } \\
\text { low point }\end{array}$ & $\begin{aligned} a & =f / 2 l \\
y^{\prime} & =\sinh x / h \\
h & =H / w\end{aligned}$ & $\begin{aligned} a & =f / 2 l \\
y^{\prime} & =x / h \\
h & =H / w \\
& =l^{2} / 2 f \\
& =l / 4 a \\
& =f / 8 a^{2}\end{aligned}$ \\
\hline $\begin{array}{l}\text { Sag } f \\
\text { Half length } s \text { of } \\
\text { cable }\end{array}$ & $\begin{aligned} f & =h(\cosh l / h-1) \\
s & =\sqrt{b^{2}-h^{2}} \\
& =\sqrt{f^{2}+2 h f} \\
& =\sqrt{2 f b-f^{2}}\end{aligned}$ & $\begin{aligned} f & =l^{2} / 2 h \\
s & =\frac{l}{2 h} \sqrt{h^{2}+l^{2}}+\frac{h}{2} \times\left[\log _{e}\left(l+\sqrt{h^{2}+l^{2}}\right)-\log _{e} h\right] \\
& =\frac{l}{2 h} \sqrt{h^{2}+l^{2}}+\frac{h}{2} \sinh ^{-1} \frac{l}{h} \\
& \approx l\left(1+\frac{8}{3} a^{2}-\frac{32}{5} \alpha^{4}+\frac{256}{7} a^{6}-\cdots\right)\end{aligned}$ \\
\hline $\begin{array}{l}\text { Vertical component } V \\
\text { of cable tension }\end{array}$ & $\begin{aligned} V & =w \sqrt{b^{2}-h^{2}} \\
& =w \sqrt{f^{2}+2 f h} \\
& =w \sqrt{2 f b-f^{2}} \\
& =w s\end{aligned}$ & $\begin{aligned} V & =w \sqrt{2 f h} \\
& =w l \\
& =4 w a h\end{aligned}$ \\
\hline $\begin{array}{l}\text { Horizontal } \\
\text { component } H \text { of } \\
\text { cable tension }\end{array}$ & $\begin{aligned} H & =w h \\
& =w(b-f)\end{aligned}$ & $\begin{aligned} H & =w h \\
& =w l^{2} / 2 f \\
& =w l / 4 a \\
& =w f / 8 a^{2}\end{aligned}$ \\
\hline Cable tension $T$ & $T=w b$ & $\begin{aligned} T & =w \sqrt{h^{2}+l^{2}} \\
& =w \sqrt{2 f h+h^{2}} \\
& =w h \sqrt{1+16 a^{2}}\end{aligned}$ \\
\hline
\end{tabular}

Fig. A1 Equations for Parabolic Cables

\section{A.1 Bending Moment Evaluation Protocols}

\section{A.1.1 Theoretical Bending Moment Evaluation Based on a Tie Rod with Lateral Loading}


A schematic diagram of tie rod in tension with a lateral load is shown in Fig.A2.

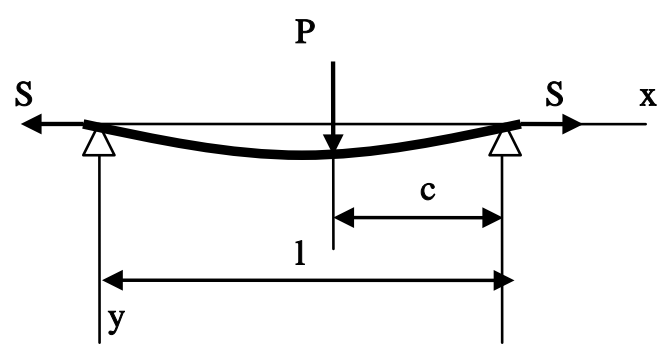

Figure A2 Simple support beam with axial tension loading

The differential equation of the deflection curve for the two portion of the tie rod, to the left and right of the unit lateral loading, $\mathrm{P}$, are

$$
\begin{aligned}
& E I \frac{d^{2} y}{d x^{2}}=S y-\frac{P c}{l} x, \\
& E I \frac{d^{2} y}{d x^{2}}=S y-\frac{P(l-c)}{l}(l-x)
\end{aligned}
$$

where $\mathrm{d}^{2} \mathrm{y} / \mathrm{dx}^{2}$ is the curvature $k$, and $E I k$ is the bending moment at $\mathrm{x}$.

using the notation $S / E I=-p^{2}$, and $S I^{2} / 4 E I=-u^{2}$, we obtain the displacement y for the left-hand portion of the tie rod in Fig. 2,

$$
y=-\frac{P \sinh p c}{S p \sinh p l} \sinh p x+\frac{P c}{S l} x
$$

A more detailed evaluation can be referred in Timoshenko’s Strength of Materials.

Considering a uniformly lateral loaded tie rod, by integrating equation (2) w. r. t uniform lateral load, we obtain the following expression for the deflection curve,

$y=\frac{w}{S p^{2}}\left[\frac{\cosh \left(\frac{p l}{2}-p x\right)}{\cosh \frac{p l}{2}}-1\right]+\frac{w}{2 S} x(l-x)$

where $w$ is the unit weight or the uniform lateral load of the tie rod.

The maximum bending moment, which in this case is at the middle of the span, is 


$$
M_{\max }=-E I\left(\frac{d^{2} y}{d x^{2}}\right)_{x=i / 2}=\frac{w l^{2}}{8} \cdot \frac{2(\cosh u-1)}{u^{2} \cosh u}=\frac{w l^{2}}{8} \cdot \frac{2}{u^{2}}\left(1-\frac{1}{\cosh u}\right)
$$

\section{A.1.1.2 Simple Support Beam Theory and Curvature Moment Approach}

\section{Simple Beam Theory}

For a simple support hinged-ends beam structure as shown in Fig. A3, at equilibrium state, all the forces should be balanced; if we take moment about mid-span point, as shown Fig. 6, the summation of all the moment should be zero about the point at the mid-span, i.e. $\Sigma$ moment about mid-span point $=0$

$=\mathrm{M}_{\mathrm{MID}}+\mathrm{H} * \mathrm{f}+(\mathrm{W} / 2) *(\mathrm{~L} / 4)-(\mathrm{W} / 2) * \mathrm{~L} / 2=0$

This leads to the bending moment formulation at the mid-span, as stated below.
Free Body Diagram

Section between mid-span and the suspension end

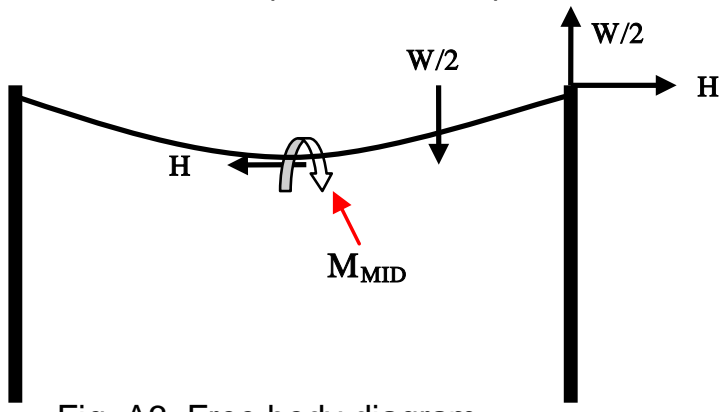

Fig. A3 Free body diagram.

$\mathbf{M}_{\mathrm{MID}}=\mathrm{WL} / 8-\mathrm{H}^{*} \mathrm{f}$

where $\mathrm{W}$ is the cable total weight and $\mathrm{f}$ is the sag at the mid-span.

An iteration scheme was used in bending moment evaluation:

(1) Assume the initial value of the H-force and then calculate the associated parabolic parameter according to parabolic equation provided in Fig. A1,

(2) Check the difference between the estimate H-force and the initial assumption, if within the allowance, then stop iteration and calculate the bending moment; otherwise use the estimated H-force back to Step 1.

\section{Cable Curvature Moment Theory}

This approach is directly related the cable bending moment to the cable curvature, on a specific geometry assumption, such as parabola or catenary geometry, as described below.

\section{Moment $=\mathrm{EI} * \mathrm{k}($ curvature $)$}

For a parabolic shape cable the curvature can be written as $\mathrm{w} / \mathrm{H}$, where $\mathrm{w}$ is the unit weight and the $\mathrm{H}$ is the horizontal tension force as shown in Fig. A3.

Table A1 lists the details of utilizing these three approaches for evaluating the cable bending moment, where the parabolic geometry of the cable structure is assumed. Different cable spans and the associated cable tension loadings were used in the bending moment evaluation. The bending moment results generated from these three approaches are very similar, and all the results show small cable bending moments exist at the middle span for variety span and cable tension loading. Nevertheless, low tension data normally show larger bending moment than the high tension data, and the high temperature data also show higher bending moments. 


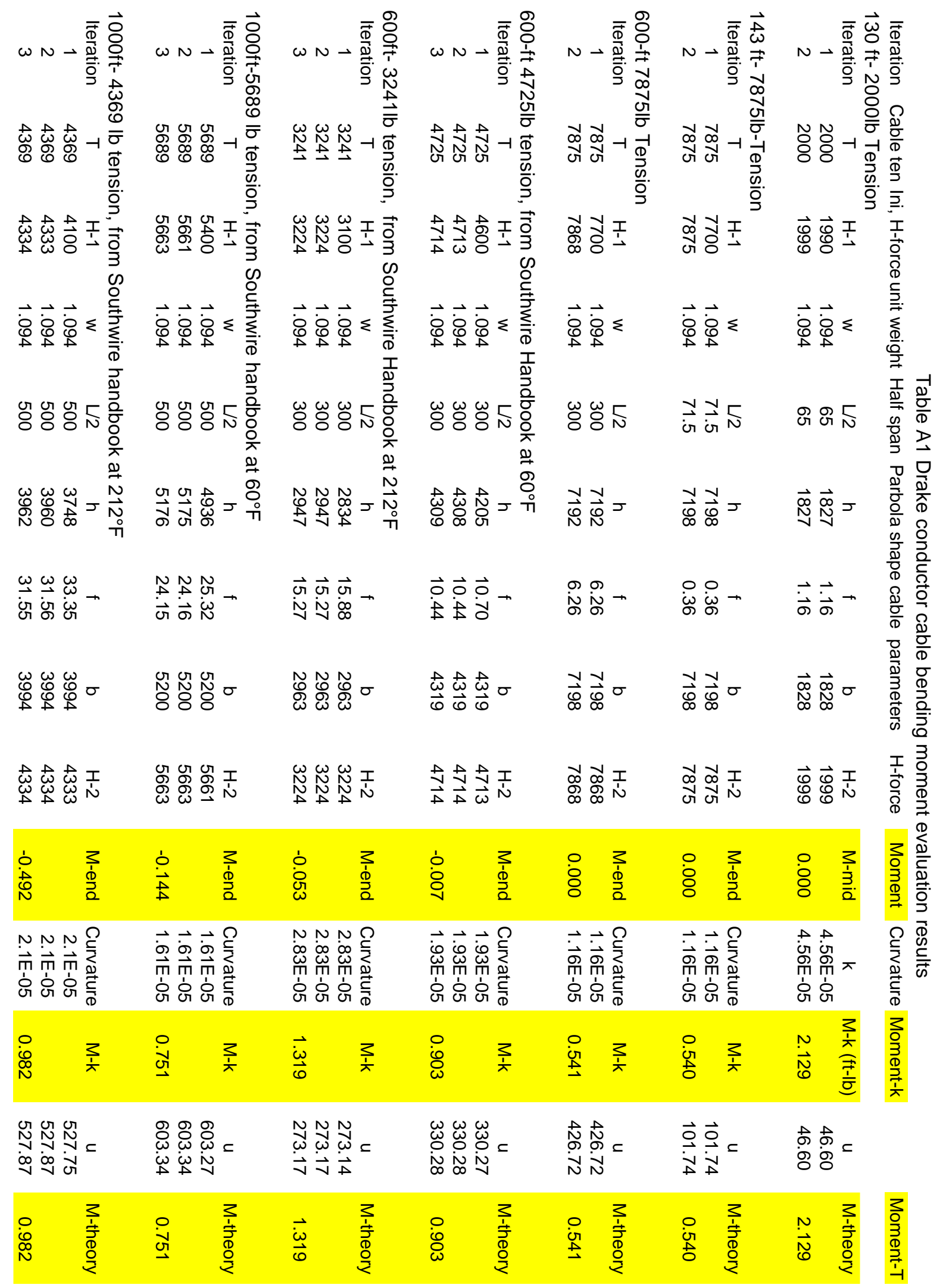




\section{A.2 Impact of Boundary Conditions to Cable Bending Moment Evaluation}

From the sensitivity study of cable bending moment investigation provided in the last section, the details of cable geometry, boundary condition and its associated loading condition all have significant impact to the cable bending moment evaluation. At here, more insight regarding the loading boundary condition induced relative rigidity changes and its impact to cable bending moment evaluation will be discussed. A high tension loaded cable will significantly increase the cable rigidity to resist the bending deformation, which was illustrated in Table A1. The higher tension loading cable shows much less sag and the bending moment as compared to that of the lower tensile loading cable. This is because that the portion of the cable dead weight induced bending moment was counterbalanced by the horizontal tensile loading; and a higher tensile loading can significantly reduce or neutralize the dead weight induced bending moment to the cable structure. This is further elaborated in the following example; Fig A4 shows several different deformations associated with their unique boundary conditions, such as different tensile loading conditions.

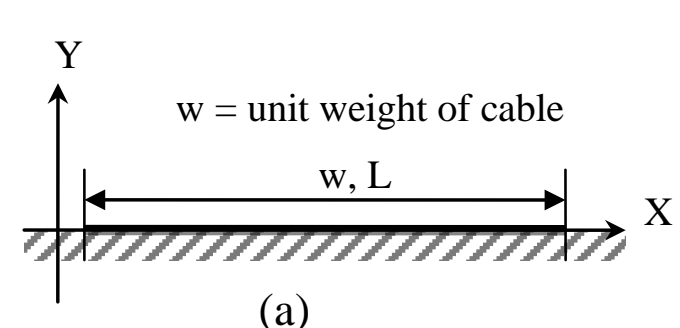

(a)

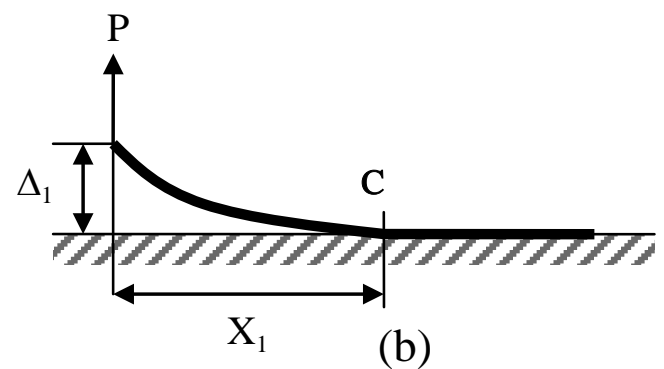

For demonstration purpose, the loading conditions are set as:

$\mathrm{P}<0.5 \mathrm{wL}$ $\mathrm{H}_{1}<0.5 \mathrm{wL} \mu$, where $\mu$ is static friction coefficient $\mathrm{H}_{2}>0.5 \mathrm{wL} \mu$

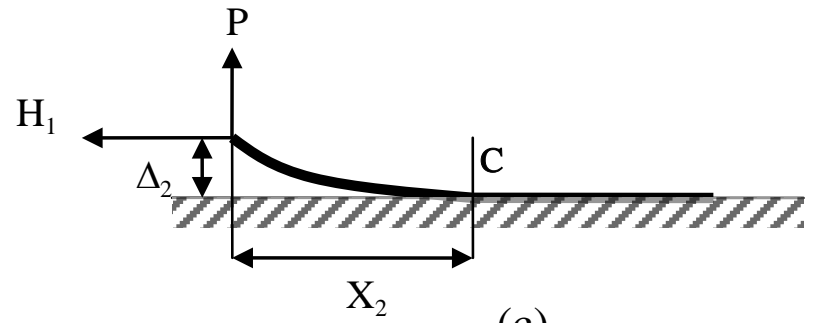

(C)

$\mathrm{X}_{\mathrm{i}}$ are the distances to the left end, where the cable was lifted above the ground, for different boundary conditions.

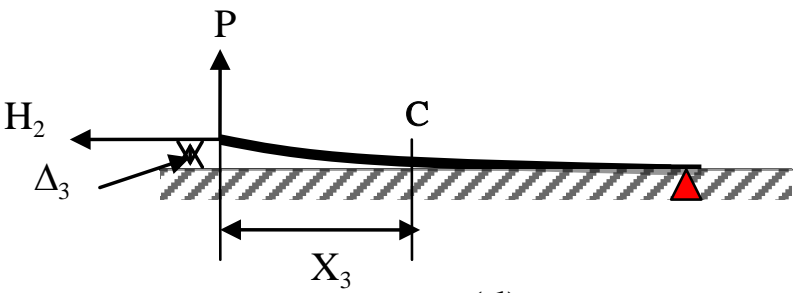

(d)

Fig. A4 Schematic diagram of a cable structure under varied boundary condition (a) cable lies on the ground, (b) Load P apply to the end of cable, (c) horizontal load $\mathrm{H}_{1}$ apply at the end of cable, (d) horizontal load $\mathrm{H}_{2}$ apply to the end of cable

Case A: Cable lies on the ground.

Fig. A4(a) shows an elastic cable lies on the ground, the dead weight was balanced by the reaction from the ground, no net shear or bending moment exists in this cable system. In other 
word, the ground boundary condition provides the extra rigidity to the cable system (or the increase of the cable system rigidity) to prevent the cable lateral deformation (in y-direction) or its bending associated deformation.

Case B: Cable lies on the ground with an uplift load P at the end of cable.

Fig A4(b) shows the same system as that of Fig. 1(a) but with a load P applied at the end of cable, which is less than half of cable total weight. The cable will be lifted above the ground at a distance $\mathrm{X}_{1}$ to the left end of cable; the corresponding bending moment will also exist in this section due to lack of lateral support from the ground. The bending moment is this section is

Bending moment $=\mathrm{Px}-\mathrm{wx}^{2} / 2$

While as the rest section (from point $\mathrm{C}$ to the right end) of cable remains in direct contact with the ground, no bending moment is existed in this region.

Due to lack of bending moment at point $\mathrm{C}$, the $\mathrm{P}$ induced bending moment, $\mathrm{P} * \mathrm{X}_{1}$ will be balanced by the dead weight load,

$\mathrm{P} * \mathrm{X}_{1}=\mathrm{w} \mathrm{X}_{1}^{2} / 2$,

from which we get distance $\mathrm{X}_{1}=2 \mathrm{P} / \mathrm{w}$. and,

the deflection at the $\mathrm{P}$ load end of the cable can be solved as

$\Delta_{1}=\mathrm{w} \mathrm{X}_{1}^{4} / 24 \mathrm{EI}$. The maximum bending moment is $\mathrm{P}^{2} / 2 \mathrm{w}$ and is located at $\mathrm{X}_{1} / 2$.

Case C: Cable lies on the ground with an uplift load P and a small horizontal tensile load.

A horizontal tensile loading was added into Fig A4(b), as shown in Fig 1(c), the resulting cable bending moment in the section between the left end and point $\mathrm{C}$ is

Bending moment: $\mathrm{Px}-\mathrm{wx}^{2} / 2-\mathrm{H}_{1} \mathrm{y}$

From equation 2, the horizontal tension loading has the effect of reducing the overall bending moment in cable by the amount of $\mathrm{H}_{1 *} \mathrm{y}$ as compared to that of equation 1 in the Case $\mathrm{B}$. Therefore, the horizontal tensile loading has the same function or similar effect as that providing by the supporting ground in Case A, to reduce or neutralize the cable bending moment induced by the P loading; and the consequently results in less lateral deformation or the bending associated deformation, i.e., the increase of the relative rigidity of the cable structure.

Due to lack of bending moment at point $\mathrm{C}$, the $\mathrm{P}$ load induced bending moment to the cable, $\mathrm{PX}_{2}$, will be balanced by that induced by the dead weight load of cable and the horizontal tensile load $\mathrm{H}_{1}$, i.e.,

$\mathrm{P} * \mathrm{X}_{2}=\mathrm{w} \mathrm{X}_{2}^{2 / 2}+\mathrm{H}_{1}^{*} \Delta_{2}$

The $\mathrm{X}_{2}$ distance will be shorter than that of $\mathrm{X}_{1}$, due to less cable weight required to balance bending moment induced by load $\mathrm{P}$ as shown in the equation 3 . It is also not difficult to visualize that the consequence of reduced lifted distance, $\mathrm{X} 2$ compared to $\mathrm{X}_{1}$, is the reduced lateral displacement at the loading end, $\Delta_{2}$, compared to $\Delta_{1}$. 
Case D: Cable lies on the ground with an uplift load P and a high horizontal tensile load.

As the horizontal tension loading continuing to increase, it will eventually result in a horizontal movement of the cable that lies on the ground. And this will require a hinge end boundary condition to stop the cable movement; the hinge end is marked with red highlight in Fig A4(d). The resulting cable bending moment in the section between the left end and point $\mathrm{C}$ is

Bending moment: $\mathrm{Px}-\mathrm{wx}^{2} / 2-\mathrm{H}_{2} \mathrm{y}$

Due to lack of bending moment at point $\mathrm{C}$, the loading $\mathrm{P}$ induced bending moment to the cable, $\mathrm{PX}_{3}$, will be balanced by that induced by the dead weight load of cable and the horizontal tensile load $\mathrm{H}_{2}$, i.e.,

$\mathrm{P} * \mathrm{X}_{3}=\mathrm{w} \mathrm{X}_{3}^{2 / 2}+\mathrm{H}_{2}^{*} \Delta_{3}$

Due to the increase of $\mathrm{H}_{2}$ as compared to $\mathrm{H}_{1}$, this will result in a further reduction of $\mathrm{X}_{3}$ and $\Delta_{3}$ compared to $\mathrm{X}_{2}$ and $\Delta_{2}$ in Case $\mathrm{C}$.

However, it is noted here for a large horizontal tension load $\mathrm{H}$, said $\mathrm{H}$ is 20 times the load $\mathrm{P}$, in order to have a balanced state of equation 5 , the cable will have a small deflection $\Delta_{3}$ and the cable will also likely be completely lifted above the ground. This will result in a very small lateral deformation (or small curvature) with $\mathrm{X}_{3}$ equals to the total length of the cable Ultimately, for a very large ratio of the tensile loading to the loading $\mathrm{P}$, the cable will likely remains straight, and the effect of the Load $\mathrm{P}$ induced deformation to the overall cable deformation is negligible.

\section{Simple Hinged-End Cable Suspension Structure}

It is not difficult to visualize that the above example can be easily transformed into a conductor cable structure with a specific boundary condition. Such as for a cable structure that is suspended on two hinge-ends, the schematic diagram of a hinged cable structure is illustrated in Fig. A5, where the load $\mathrm{P}$ is equal to the half of cable total weight (wL/2), i.e., $\mathrm{P}$ is induced by the cable total weight. The sag $f$ of the cable is roughly equivalent to the end deflection,

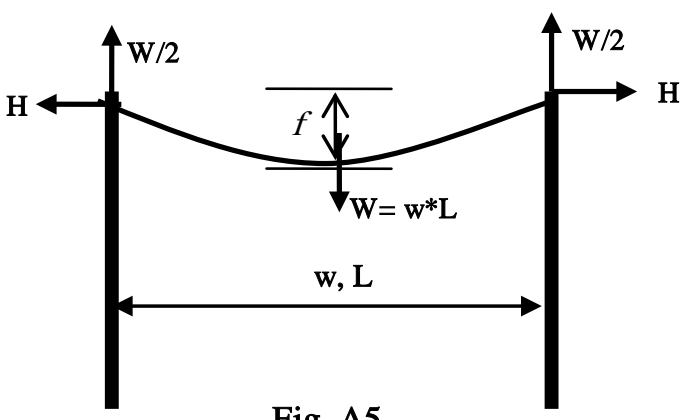
$\Delta$, in Fig. A4.

The associated bending moment at the center of the cable in Fig. A5 can be expressed as $\mathrm{M}_{\mathrm{MID}}=(\mathrm{wL} / 2) *(\mathrm{~L} / 2)-(\mathrm{wL} / 2) *(\mathrm{~L} / 4)-\mathrm{H}^{*} f$ $=\mathrm{WL} / 8-\mathrm{H}^{*} f$

From equation 6 , the horizontal tension loading has the effect of reducing the bending moment induced by cable total weight ( $\mathrm{WL} / 8$ ) with the amount of $\mathrm{H}_{*} f$. Therefore, for a fixed cable span, a higher tension loading will result in a less bending moment and deflection than that of lower tension loading case. This scenario was also demonstrated in the earlier bending evaluation shown in Table A1. 
Therefore, the cable horizontal tensile loading, which has the same function or similar effect as that of the supporting ground as demonstrated in Fig 1, provides a mechanism to reduce or neutralize the cable bending moment induced by the cable dead weight loading, and results in a less lateral deformation (or a less deformation associated with bending moment loading). Thus, in an equivalent term, the tensile loading within a conductor cable structure will provide an increase in the relative rigidity of the cable structure to resist the cable deformation associated with the cable bending moment induced by the cable dead weight. 



\section{$\boldsymbol{B}$ MIXED-MODE LOADING CONDITION OF A TWISTED CONDUCTOR WIRE BUNDLE}

\section{B.1 Combined Stress States of a Helical Wire Under Tension}

A mixed-mode loading condition, tensile and flexural normal stress plus shear stress, will occur within a helical wire under tension loading along the central axial of the helical wire. For demonstration purpose, one assumes that the wire diameter is much less than the radius of the helix. The geometry of a helical wire is illustrated in Fig. B1. At any point of a helical wire, point $\mathbf{A}$, the tangent to the helical center line of the wire is not parallel to the tensile loading $\mathrm{P}$. Therefore, the tensile force $\mathrm{P}$ will produce a bending moment (about the axis $\mathrm{n} 1$ ) and a torque at the cross section of $\mathbf{A}$ on a helical wire, in addition to tensile loading at $\mathrm{A}$. The force $\mathrm{P}$ is resolved into two components: $\mathrm{P}^{*} \cos \theta$, perpendicular to the tangent of helical wire at $\mathbf{A}$, and $\mathrm{P}^{*} \sin \theta$, parallel to the tangent of the helical wire at $\mathbf{A}$. At cross section of point $\mathrm{A}$ the component $\mathrm{P}^{*} \cos \theta$ produces the torque $\mathrm{T}=\mathrm{P} * \mathrm{R} * \cos \theta$, where $\mathrm{R}$ is the radius of the helix, and the component $\mathrm{P} \sin \theta$ produces the bending moment $\mathrm{M}=\mathrm{P}^{*} \mathrm{R}^{*} \cos \theta$.

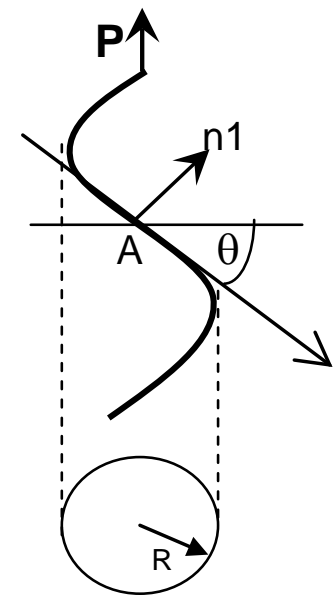

Fig. B1. Helix geometry.

The maximum combined stress is $\sigma_{\max }=16\left(\mathrm{M}+\sqrt{ }\left(\mathrm{M}^{2}+\mathrm{T}^{2}\right)\right) / \pi \mathrm{d} 3=16 \mathrm{PR}(1+\sin \theta) / \pi \mathrm{d} 3$, where $\mathrm{d}$ is the diameter of the wire. The maximum shear is $\tau_{\max }=16\left(\sqrt{ }\left(\mathrm{M}^{2}+\mathrm{T}^{2}\right)\right) / \pi \mathrm{d} 3=16 \mathrm{PR} / \pi \mathrm{d} 3$. This exercise may indicate that the outer layer of the conductor cable has relative larger helix radius and will experience larger normal and shear stresses than that of inner layer of the conductor cable. However, the magnitude of these stress fields is expected to be significantly reduced in a conductor cable as compared to that of a single helical wire. This is because the lateral support to the helical wire was received from the adjacent twisted conductor wires reducing the associated bending moment and torque induced by the helical wire geometry. This condition was further investigated with a more detailed finite element analysis as illustrated below.

\section{B.2 Finite Element Analysis of Conductor Cable with Helical Twisted Wires Under Tension}

This Finite Element Analysis (FEA) focused on two kinds of overhead conductors - one is 636 ACSR (Aluminum Conductor, Steel-Reinforced) and another is 636 ACSR/TW (Aluminum Conductor, Steel-Reinforced/Trapwire). ACSR consists of a solid or stranded galvanized steel core surrounded by one or more layers of 1350-H19 aluminum (Fig. B2). ACSR/TW differs from conventional ACSR in that its aluminum strands are trapezoidal shaped (Fig. B2). Both 
conductors are used extensively on long spans as both ground and phase conductors because of their high mechanical strength-to-weight ratio and good current-carrying capacity.

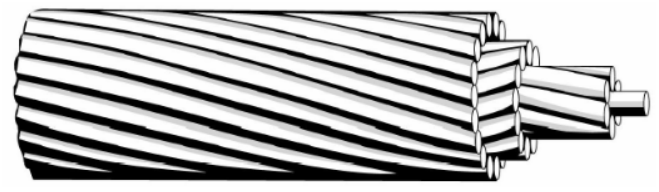

Aluminum Conductor Steel Reinforced (ACSR)

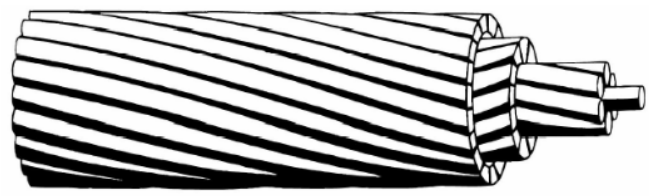

Aluminum Conductor Steel Reinforced Trapezoidal Shaped Aluminum (ACSR/TW)

Figure B2. Schematic drawing of ACSR and ACSR/TW conductors

\section{B.2.1 Single Straight Wire Vs. Single Twisted Wire}

Most power conductors are in concentric-lay stranded form; that is, a single straight core wire is surrounded by one or more helically curved wires. The direction of twist of lay is usually reversed in adjacent layers. All wires of a given layer are generally the same diameter for ACSR or same shape for ACSR/TW.

Because of the helical path of the strand layers there is more length of metal in a given length of stranded conductor than in a solid round conductor of the

same AWG (American Wire Gage) size, hence both the weight and dc resistance per unit length are increased. Incremental increase for weight and dc resistance of stranded over that solid conductor is roughly $2 \%, 3 \%$ and $4 \%$ for sizes $2,000,000$ cmil or under, sizes $3,000,000$ to

$2,000,001 \mathrm{cmil}$ and sizes $4,000,000$ to 3,000,000 cmil respectively.

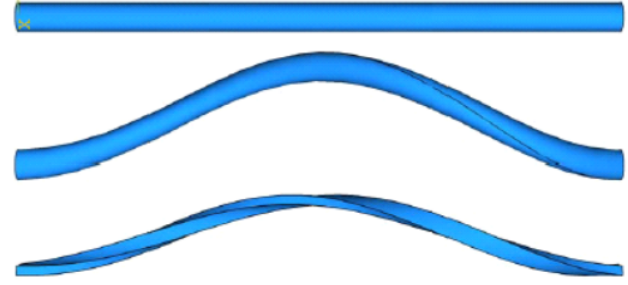

Boundary conditions:

One end $(Z=0)$ center point: $U_{X}=U_{Y}=U_{Z}=0$, blue area: $U_{Z}=0$; Other end $(Z=L)$ center point: $U_{X}=U_{Y}=0, U_{Z}=0.2 \%{ }^{*}$, blue area: $U_{Z}=0.2 \%{ }^{*} \mathrm{~L}$

Figure B3. Straight wire vs. twisted wires
Figure B3 shows the three types of wiresthe straight circular wire, the twisted circular wire and the twisted trapezoidal wire. In order to make comparison, the boundary conditions are exactly same one end fixed and the other end applied with the same displacement for all the three cases.

The straight wire has the uniform stress distribution under these boundary conditions - that is 20,000 psi along the axial direction for the 1350-H19 aluminum material $\left(\mathrm{E}=10 \times 10^{6} \mathrm{psi}\right)$. Both the circular and trapezoidal twisted wires, however, show highly nonuniform stress distribution under the same boundary conditions. Due to the concentric-lay stranding, the bending, torque and axial force all make contributions to the stress fields and the stress ranges from 170 psi to 11,000 psi (Figs. B4 and B5). It's obvious that a single twisted wire has less capacity to hold the tensile loading as compared to a straight wire under the same boundary condition. 


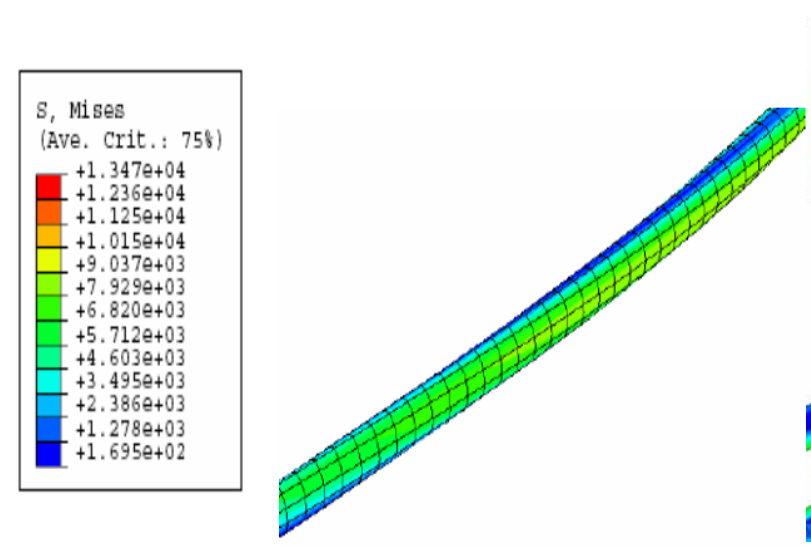

Figure B4. The Mises stress distribution of a circular twisted wire

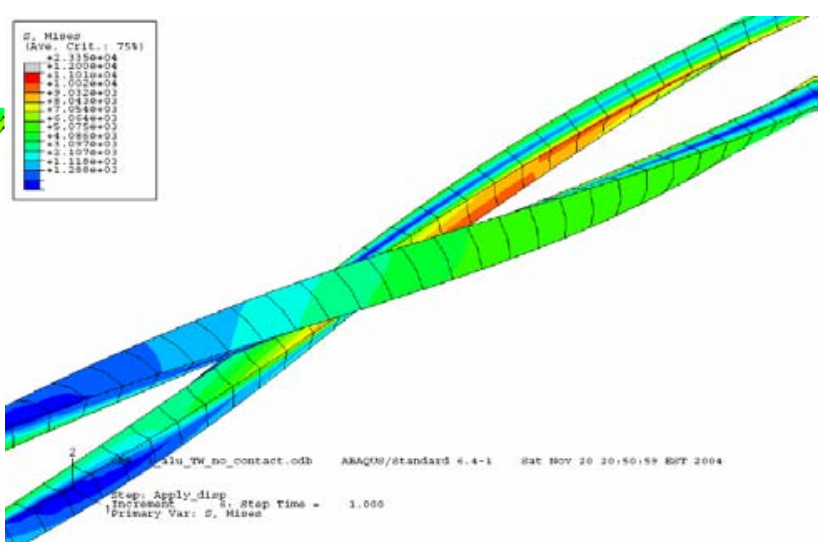

Figure B5. The Mises stress distribution of a trapezoidal twisted wire

\section{B.2.2 Conductor Cable with Bundles of Twisted Wires}

\section{Simplification of the steel core of the Conductor Cable}

ACSR conductor consists of a solid or stranded steel core surrounded by strands of aluminum (Fig. B2). There are 7 steel wires on the core for the 636 ACSR conductor. In order to mitigate the demand of the computer memory and dramatically reduce the computational time, 7 straight steel wires can be approximately represented by a single "nominal" straight steel wire with the same diameter. The aluminum wires will surround and contact this "nominal" wire and hence the number of contacted surfaces is greatly reduced.
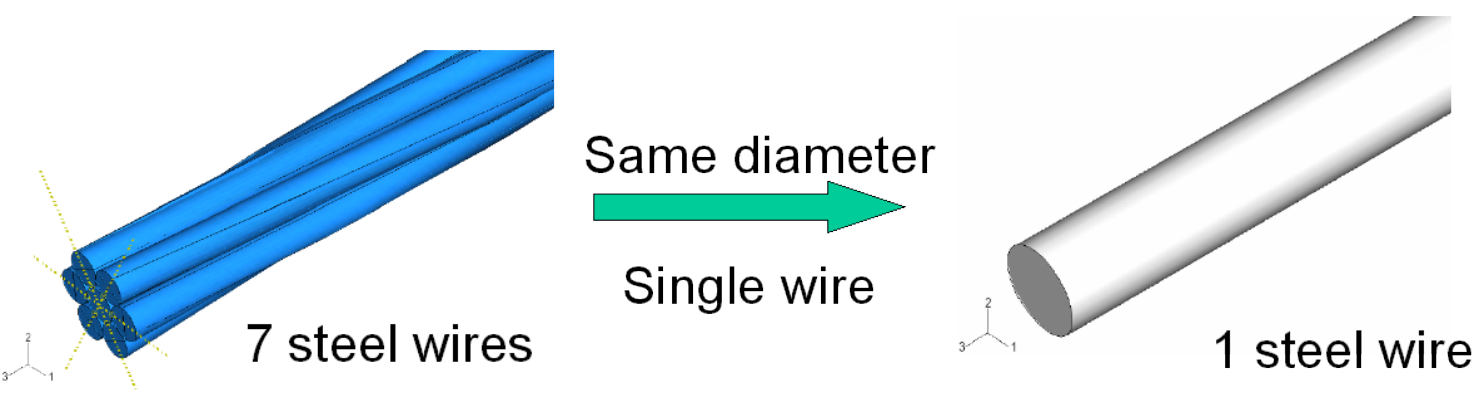

Figure B6. Simplification of the core material

\section{FEA Analysis: Non contact vs. contact between wires}

According to the previous analysis, the single twisted wire is not efficient to hold the tensile loading. The contact gives the lateral support to the wire and the wire cannot "freely" make inward movement to the centroid of the conductor. 
Figures B7 and B8 give the comparison of the wires with contact and ones without contact (in order to make comparison, the same boundary conditions at ends are used). The wires without contact constraint mean that they can "freely" move laterally without any constrains under loading. One can see that the stress distribution for the wire without contact is highly nonuniform, the Mises stress ranges from 170 psi to 11,000 psi. However, the stress distribution for the wire with contact constraint is nearly uniform, and the Mises stress varies from 17,000 psi to 19, 000 psi. The Mises stress of the straight wire with the same material under the exact same boundary conditions is 20,000 psi. The difference between the stress of the twisted wires with contact and one of the straight wires is less that $15 \%$. It means that "contact" does play a significant role in redistributing the stress. The small lateral contact forces make the stress distribution of the twisted wires more or less uniform. With contact constraint, a twisted wire can hold almost the same load as a straight wire's under the same boundary conditions.
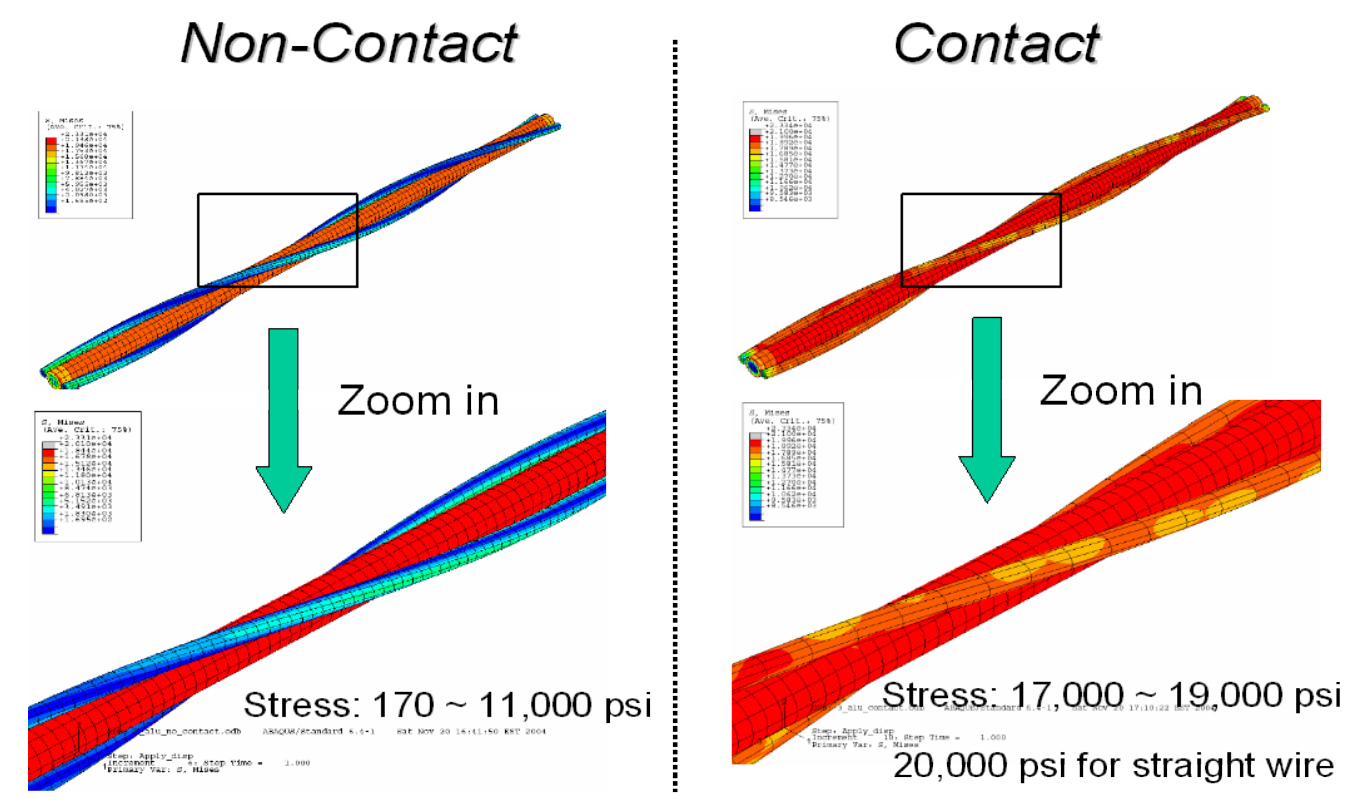

Figure B7. The comparison between the wires with contact and ones without contact for the circular wires

\section{FEA Analysis: Face contact vs. Lateral contact between Wires}

- $\quad$ Mises stress distribution under tensile loading

Depending on the size of the gaps between the wires in the adjacent layers and among the wires in the same layer, there exist at least two kinds of contacts. One is called the "face contact," where the wires between the adjacent layers make face to face contacts; the other is the "lateral contact," where the wires in the same layers make contact on their sides. Other types of contacts or combination may also exist, however, only these two kinds of contacts are studied here. No matter the face contact or the lateral contact, the stress distribution is more or less uniform (Fig. B9). However, in reality, gaps may exist between the wires even after tension loading. Therefore, the existing gaps between the wires must be fully close before any form of contact can occur. 
Hence, real stress distributions should be somewhere between the wires without any contact and the ones with full contact.
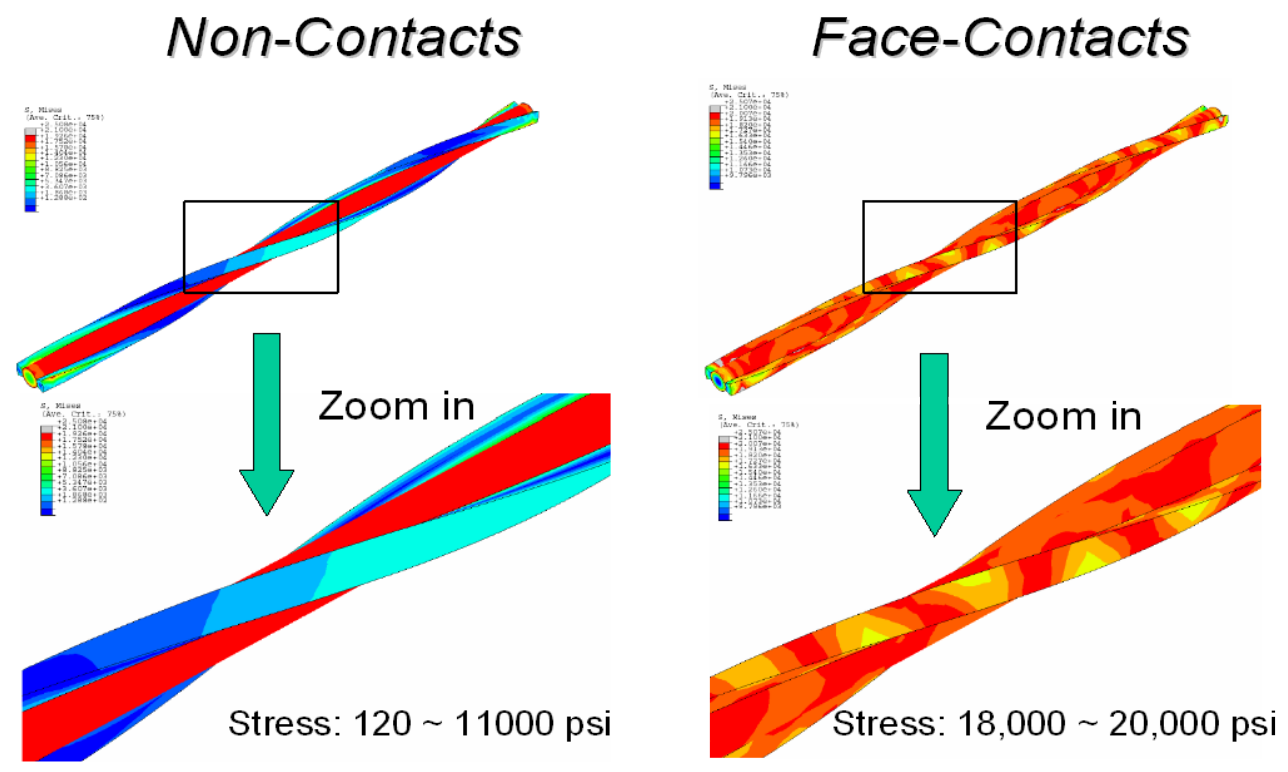

Figure B8. The comparison between the wires with contact and ones without contact for the trapezoidal wires
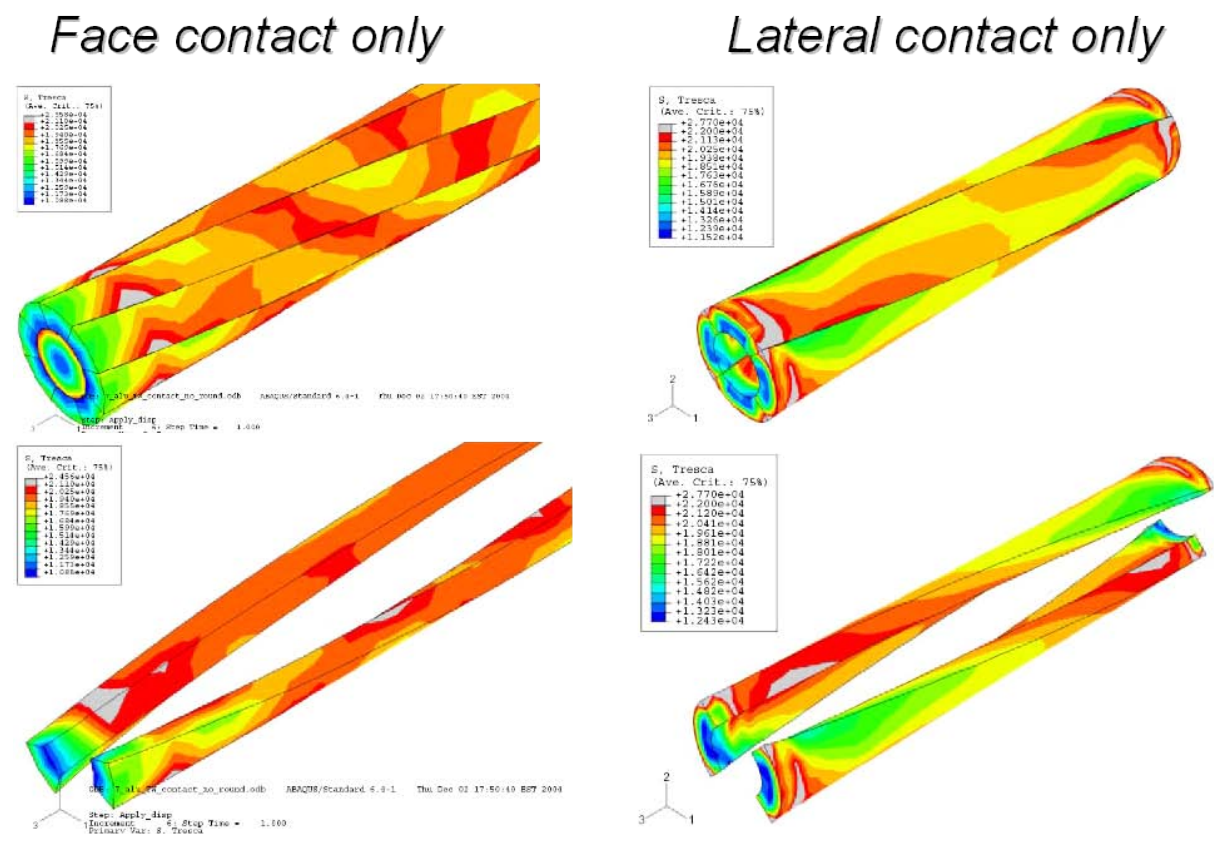

Figure B9. Face contact and lateral contact.

- $\quad$ Contact pressure and Mises stress along the contact surfaces 
Contact plays a significant role in redistributing the stress on the wires. The Contact pressure cannot be too big or non-uniform otherwise the wires will lose balance during loading. Figure B10 gives the contact pressure distribution along the contact surfaces. For the face contact, pressure ranges from 0 to 100 psi. For the "lateral contact," the contact pressure is not uniformly distributed along the contact surfaces. The closer to the internal surface, the bigger the contact pressure.
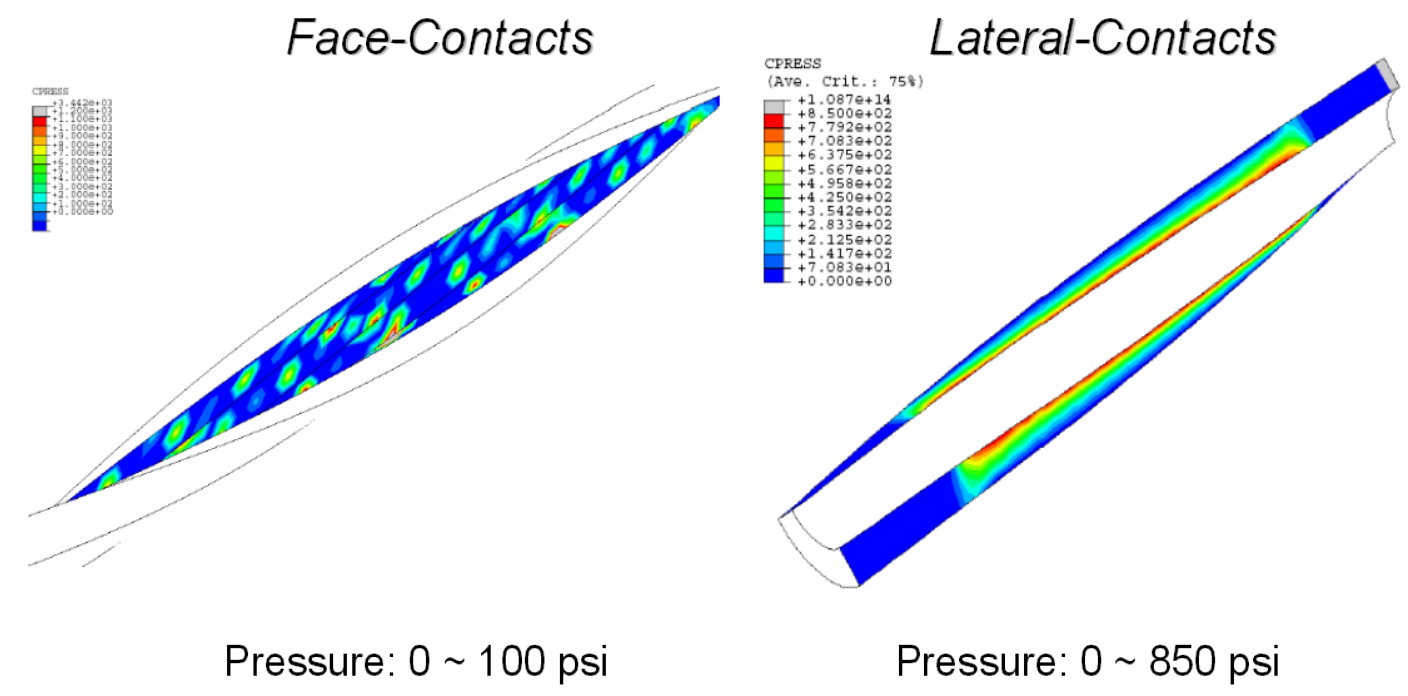

Figure B10. Contact pressure along the contact surfaces.

The Mises stress also shows some non-uniform distribution. The Mises stress along the interior line is about 5\% more than one along the exterior line (Fig. B11). The previous result also shows that the contact pressure is the highest along the interior line. It indicates that damage may initiate from the interior line where the contact pressure and stress level are the highest.

\section{FEA Analysis: Radial displacement}

After the initiation of "contact" scenarios, the twisted wires will likely move inward to reduce the existing gaps. If the gaps between the two adjacent layers are small enough, the wires may also have "face contact" in addition to the "lateral contact." With $0.2 \%$ strain for the straight wire, the radial displacement along the radius $=0.17$ inch for the trapezoidal wires is shown in Fig. B12. The total radial displacement is about $0.14 \times 10^{-3}$ inch. If the gap between two adjacent layers is less than this value, the face contact will occur after the lateral contact. In this analysis, an assumption was made that there was no gap between the lateral surfaces before loading or displacement boundary conditions were applied. However, in reality conductor cable may always have gaps between the wires before installation. If load or displacement is "gradually" applied to a conductor, the gaps should be "slowly" closed first before the wires make any contact. 


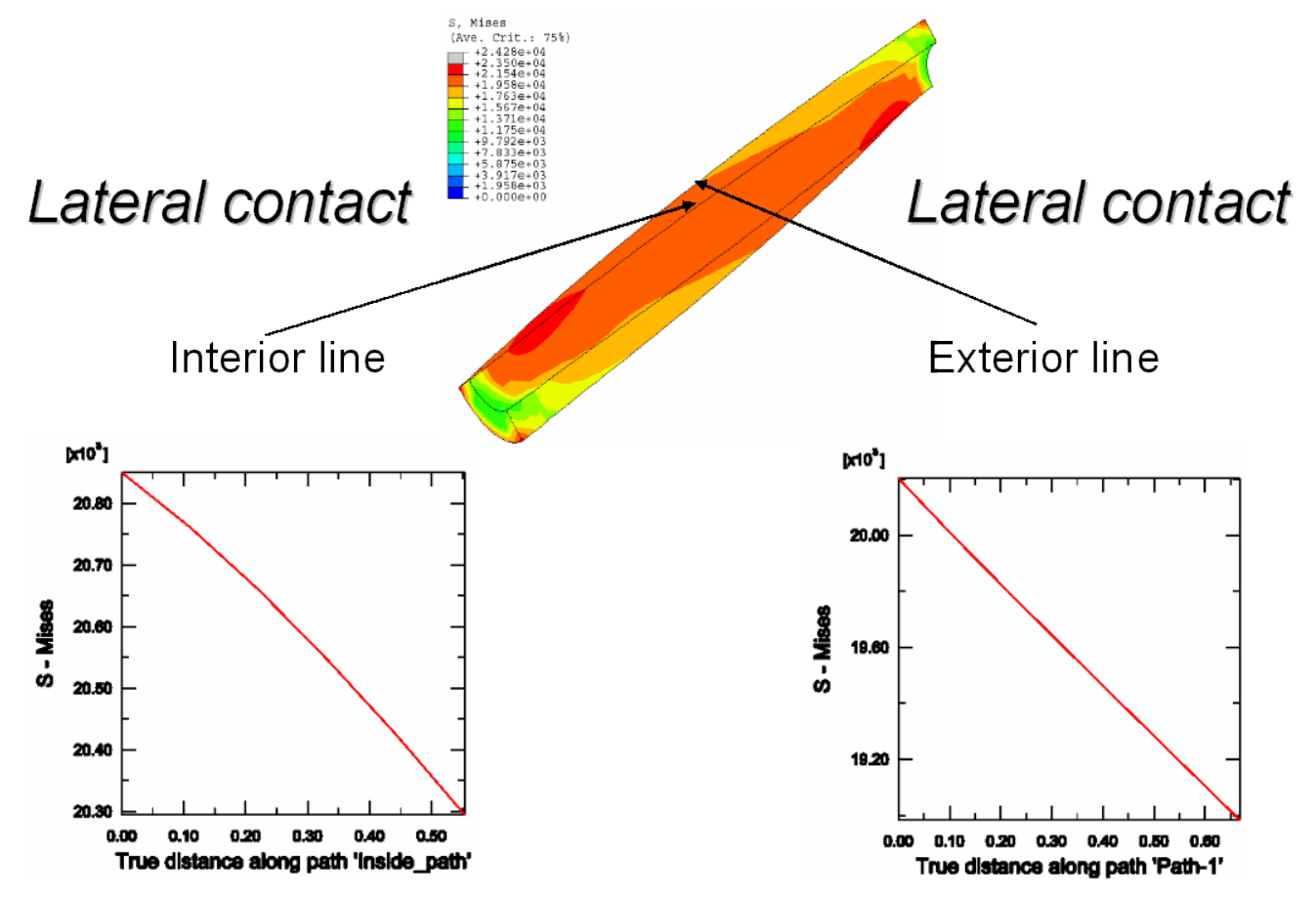

Figure B11. Mises Stress Distribution along the Edges of the contact surface.

\section{B.2.3 Conclusion and Future Work on Mixed-mode Loading Analysis}

1. Twisted wire without contacts has highly non-uniform stress distribution due to bending and twisting.

2. It is the contact pressure that plays the critical role in redistributing the stress - rendering it nearly uniform along the cross section.

3. For ACSR, contacts among wires in adjacent layers may play a more important role than ones between wires within the same layers.

4. Even though contacts significantly reduce the non-uniformity in stress distribution, there still exists a stress variation within the cable.

5. Stress usually is higher on the contact areas than on the non-contacted areas, which suggests that micro-crack may first initiate from the contact areas and subsequently propagate further.

6. Mixed-Mode fracture/fatigue analysis and accounting for nonlinear material behavior may be needed for life prediction and failure evaluation.

7. For ACSR/TW, the relative roles of lateral and face contacts depend on the gaps from existing cable design.

- Lateral contacts only: gaps between layers are relatively large 

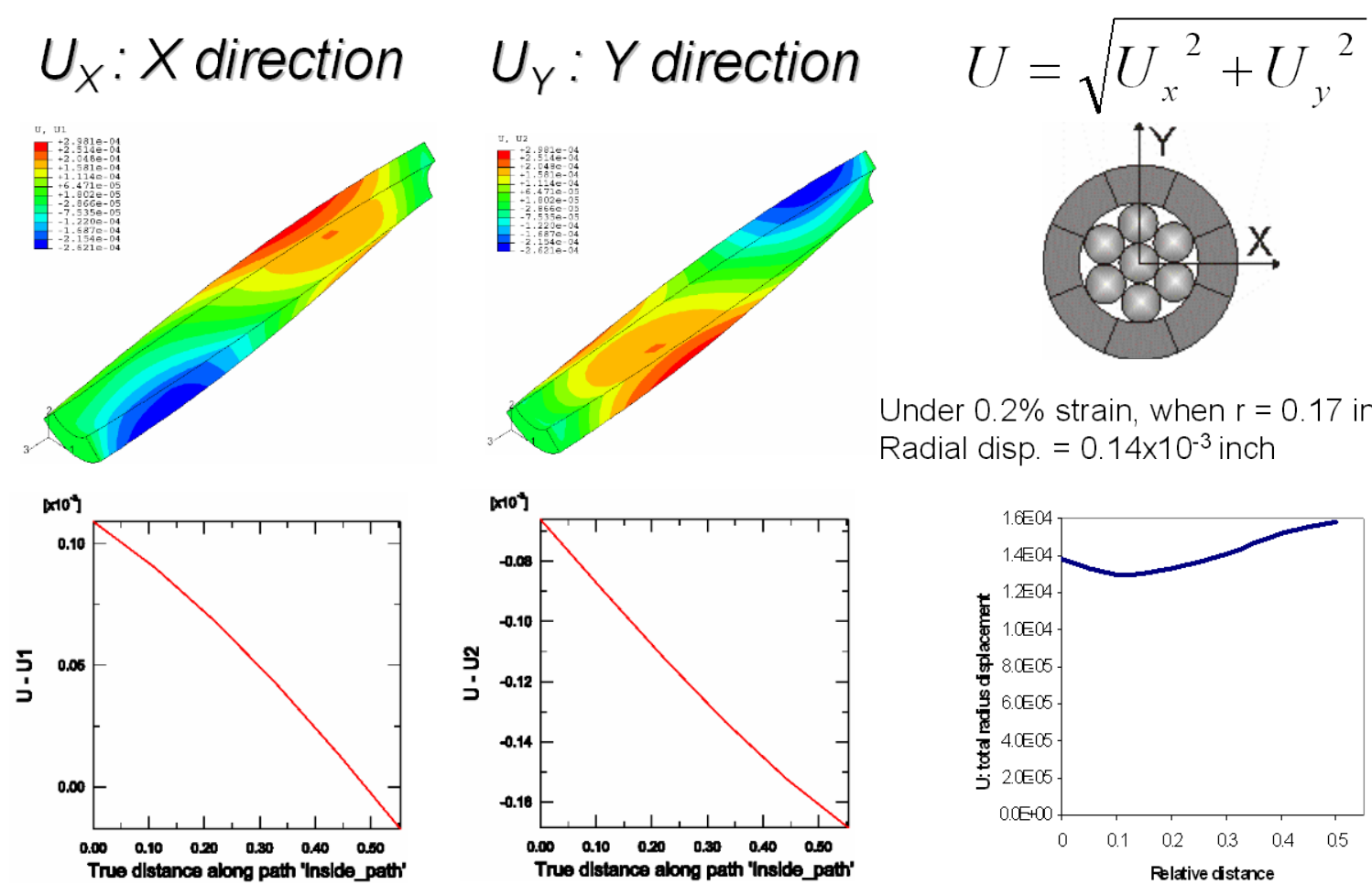

Under $0.2 \%$ strain, when $r=0.17$ inch Radial disp. $=0.14 \times 10^{-3}$ inch
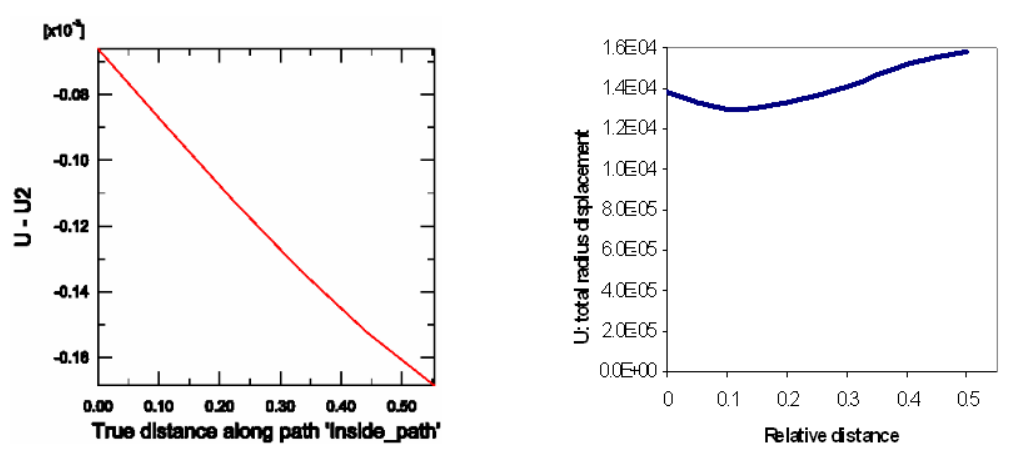

Figure B12. Radial displacement of the trapezoidal wires with the lateral nnntont

- $\quad$ Face contacts only: gaps between the wires in the same layer are big enough

- $\quad$ Lateral contacts first, then face contacts: the elevated temperature may close gaps

- $\quad$ No contacts first, then either face contacts or lateral contacts or even combination of face contacts and lateral contacts, in this case, stress distribution is fully depended on the load level, temperature as well as of gaps size; the stress distribution should be between the full-contact and non-contact scenarios.

The spin-off of this investigation may help industry to design twisted wire configuration with proper pitch angle for better performances of the conductor cable. Secondly, due to different design criteria of ACSR and ACSS, the design testing focus, such as creep or fatigue, will need to be modified accordingly. For example, ACSR is normally operated at $50^{\circ} \mathrm{C}$ to maximum, $150^{\circ} \mathrm{C}$; however, the critical operating temperature is around $125^{\circ} \mathrm{C}$. Generally, at low temperature both the aluminum conductor and the steel core both take up the loading, but at the relative higher temperature, steel strands will carry all the loading. However, as for ACSS, the conductor may be operated as high as to $200^{\circ} \mathrm{C}$, but the aluminum conduction is so call "dead soft" and will not take any loading, all the loading will be carried by the steel strands. Thus, for fatigue or creep model developments, we will need to design several models for different operating temperature scenarios. Regarding the ACSS, the lack of contact pressure between the aluminum conductor wires can create more gaps. In dynamic wind loading condition, such gaps may improve damping coefficient but the relative movement of the conductor wire will result in accelerated damage to conductor wires. 


\section{MECHANCAL TESTING RESUTSS - CREEP, FATIGUE, AND THERMAL-MECHANICAL FATIGUE}

\section{C.1 CREEP TEST STE-UP AND ATS TESTER UPGRADE}

\section{C1.1 Creep and Fatigue test samples preparation}

The specimen design for creep and fatigue testing is shown in Fig. C1. The length of the creep and fatigue specimen is 2.3 inch with gage diameter of 0.18 inch, as shown in Fig. C2. In addition to test samples made from 1350-H19 aluminum conductor material, test samples were also made from 3003-H183 splice connector sleeve and 6061-T6 core-grip. The EDM technique was used to machine the designed specimen from the cylinder thickness section of the connector sleeve and from the spike regions of the core-grip as shown in Fig. C3.

\section{ORHA FATIGESEAMEN SCALE $=21$}

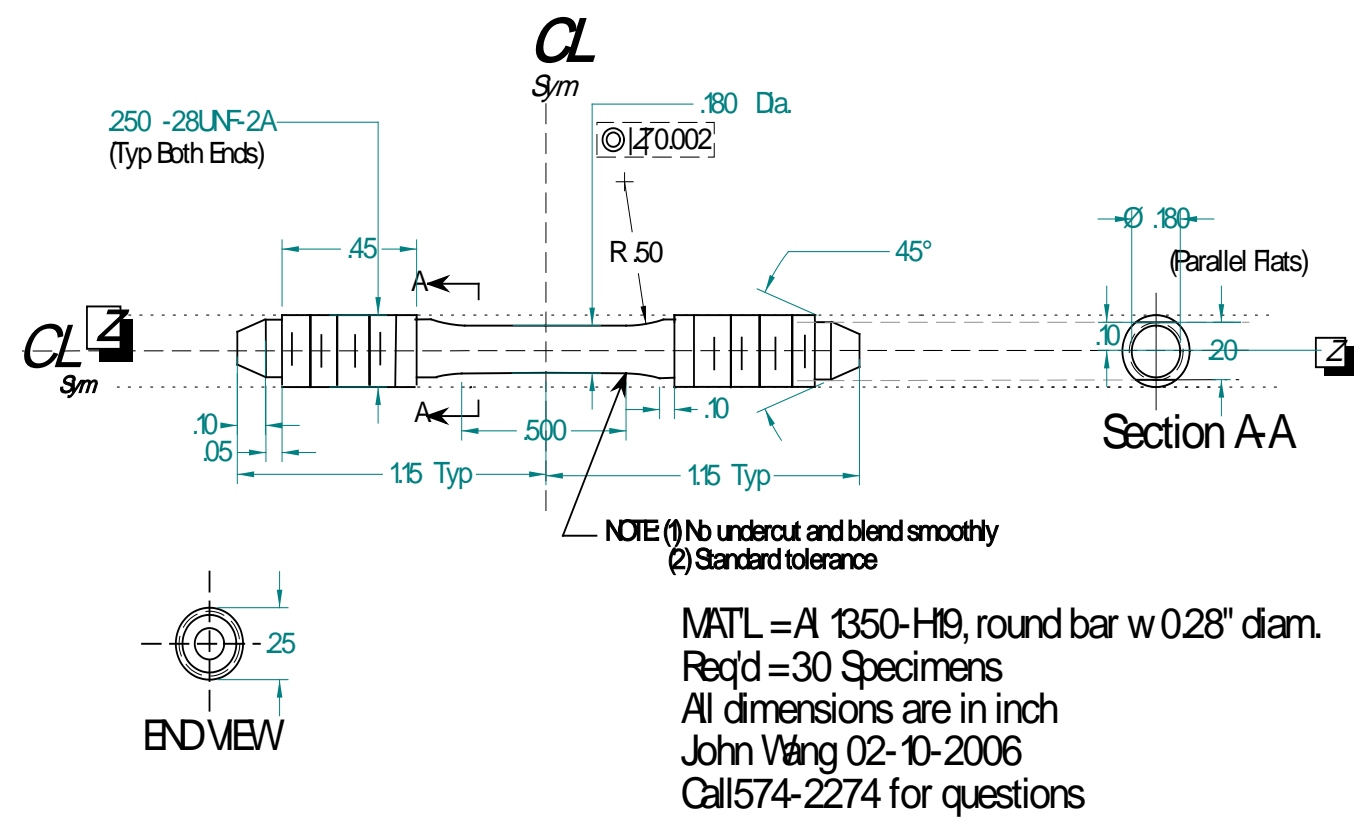

Fig. C1. Creep and fatigue specimen design configuration. 


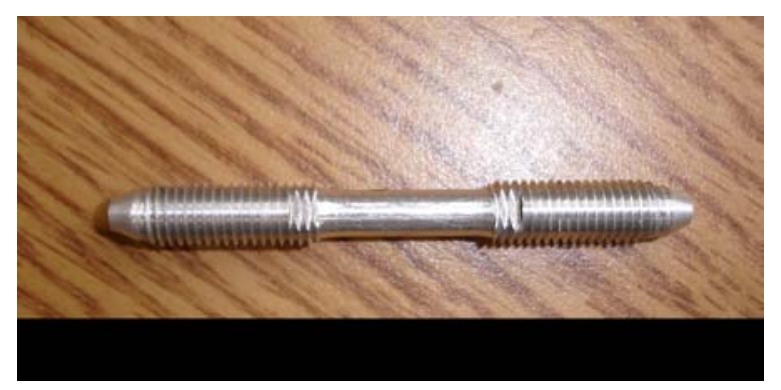

Fig. C2 Creep test specimen.

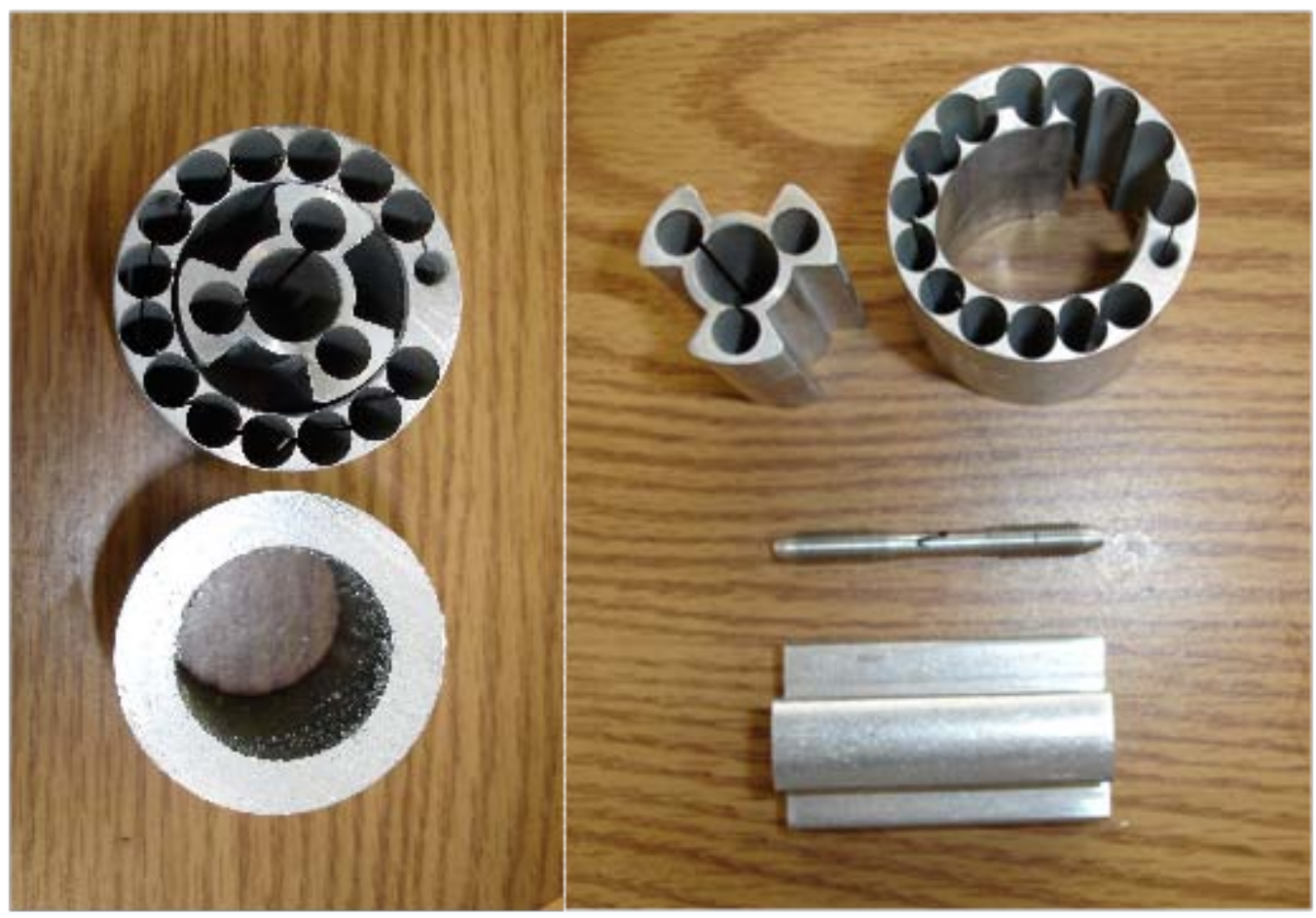

Fig. C3 Samples machined from core-grip and splice sleeve.

\section{C.2 Creep Test Set-Up and Load Train Design}

The design of the laser mike flag holder, attached to the creep test sample, for creep deformation control is shown is Fig. C4. The load train associated linking rods designs for ATS creep tester are shown in Figs. C5-C7. The linking rods designed for the dead weight creep tester is shown in Fig. C8.

The thermal couple set-up for temperature control during the creep testing is shown in Fig. C9. Due to small gage length, 0.5 inch, the thermal couple is instead attached to the end of specimen grip. The calibrated temperature bias for this thermal couple position compared to that at specimen is about $5^{\circ} \mathrm{C}$. The creep test set-up is shown in Fig. C10. Fig. C11 shows the details of Laser-Mike set up for deformation control. The LabView for I/C control and data acquisition was completed and the front end is demonstrated in Fig. C12. 


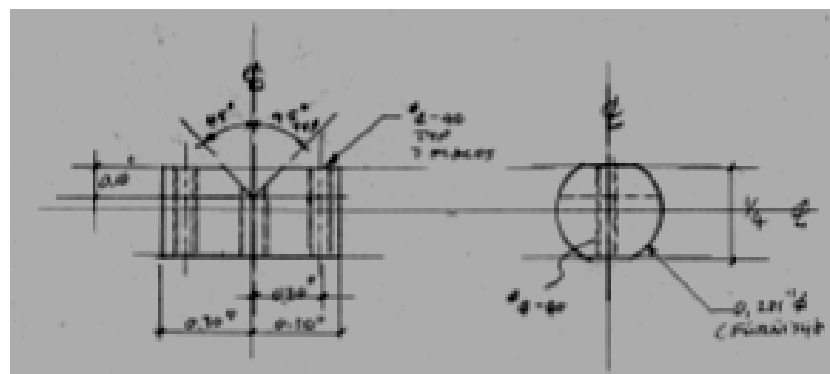

Fus intpre

Ree'o. $\operatorname{sic}(6) / a n c a s$.

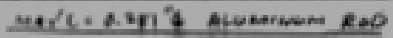

rewions oy Roewrove

Fig. C4 Laser Mike flag holder configuration.

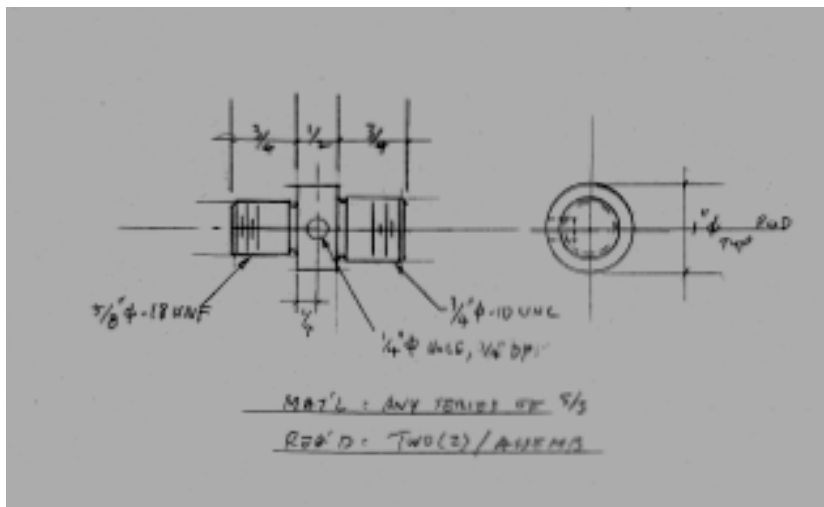

Fig. C6 ATS tester rod adapter design 1.

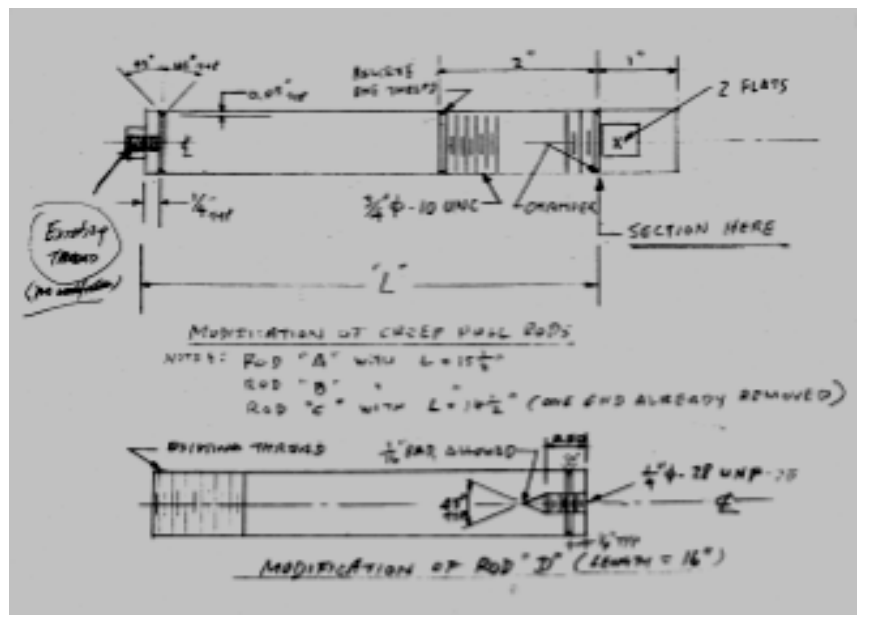

Fig. C8 Rod design for dead weight creep tester.

C-3

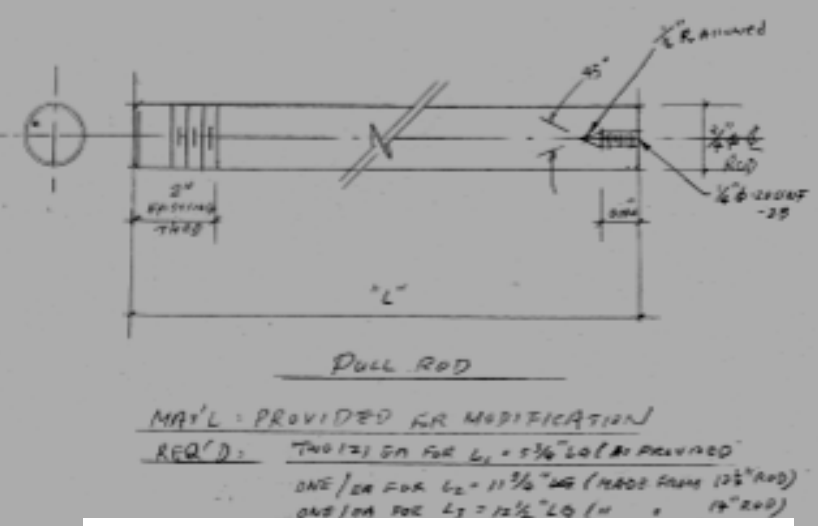

Fig. C5 ATS creep tester puller rod design.

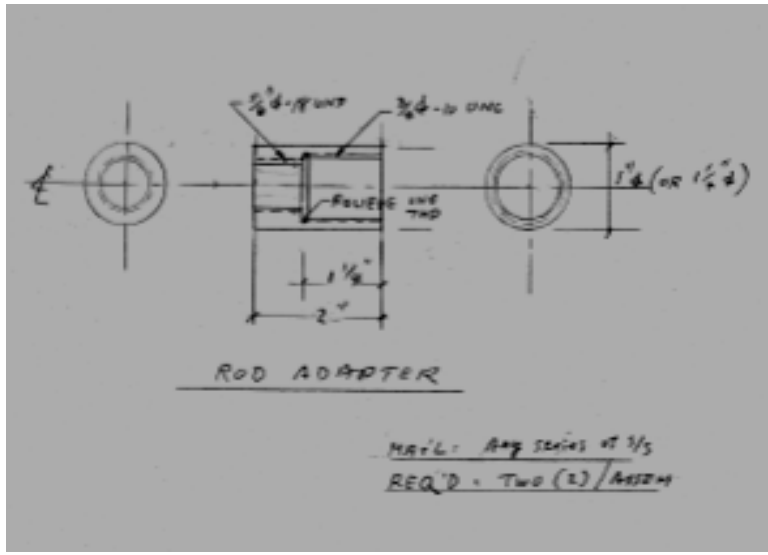

Fig. C7 ATS tester rod adapter design 2.

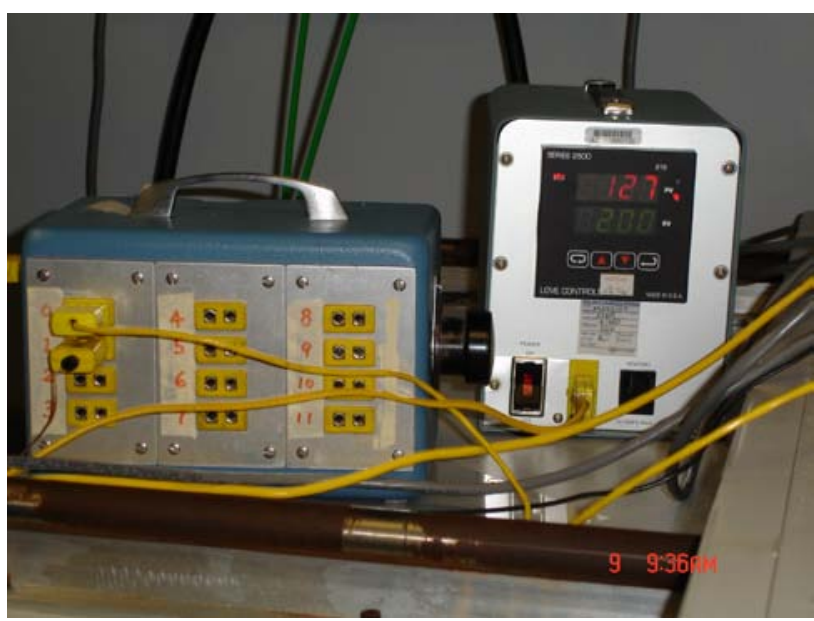

Fig. C9 Thermal couple control set-up. 


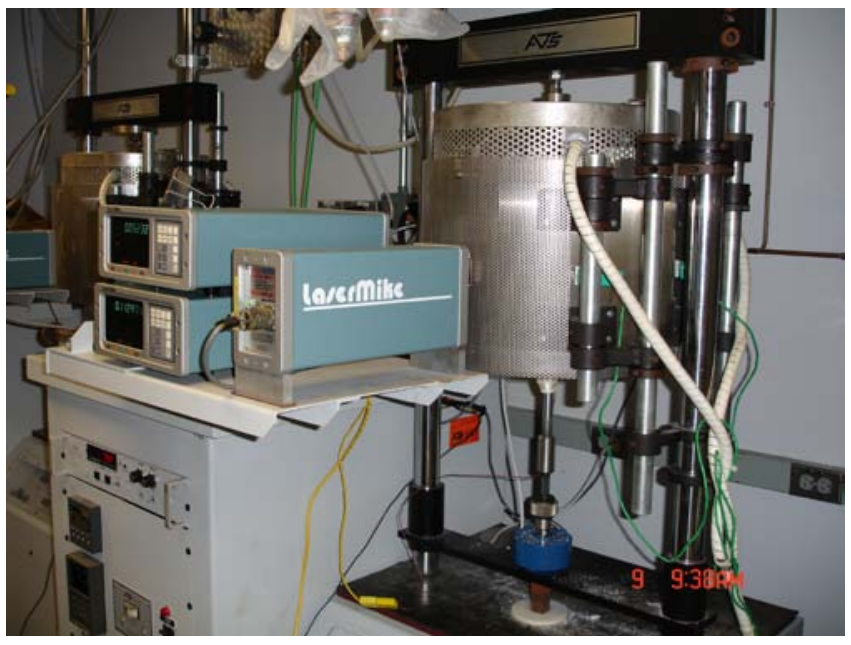

Fig. C10 Creep test set-up, including environmental chamber, load frame \& load controller, Laser Mike, and furnace control.

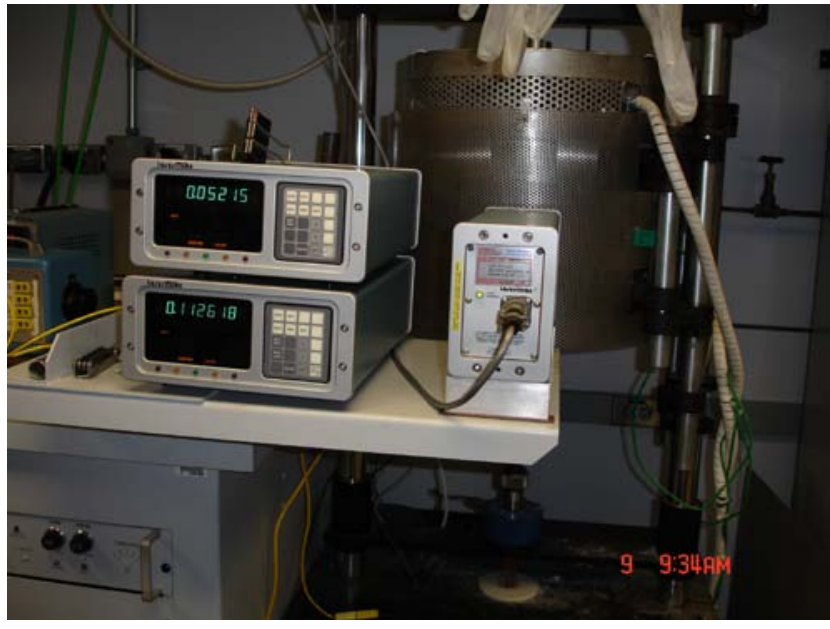

Fig. C11 Detailed view of Laser Mike set-up and its alignment with the window of furnace.

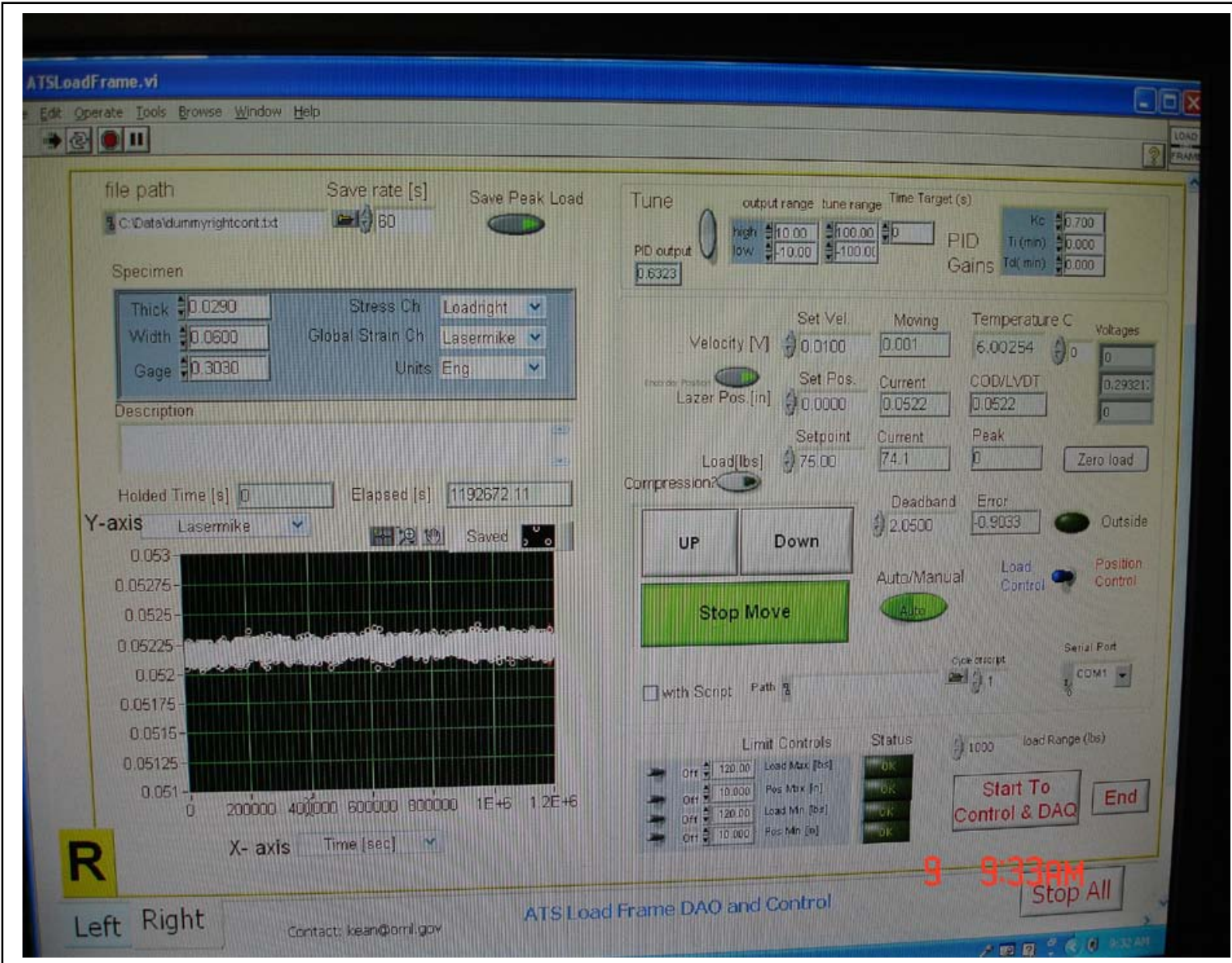

Fig. C12 The front end of LabView for ATS creep testing load control and data acquisition. The creep data collection history/per minute time interval is shown in the lower left end portion of the front end. 


\section{ATS Creep Tester and MTS 810 Tester Repairs}

One ATS creep tester controller had bad performance during creep testing. The detailed investigation found out that one of the pneumatic pumps had minor leak, this was attributed to the load oscillation during the creep testing. In order to further improve the load control, the ATS load system controller was replaced with PC control. The repaired pneumatic pump is shown in Fig. C13. The location of the severe hydraulic oil leak from the actuator of MTS 810 tester is shown in Fig. C14, it was later repaired by MTS personnel and resumed fatigue and TMF testing operation.

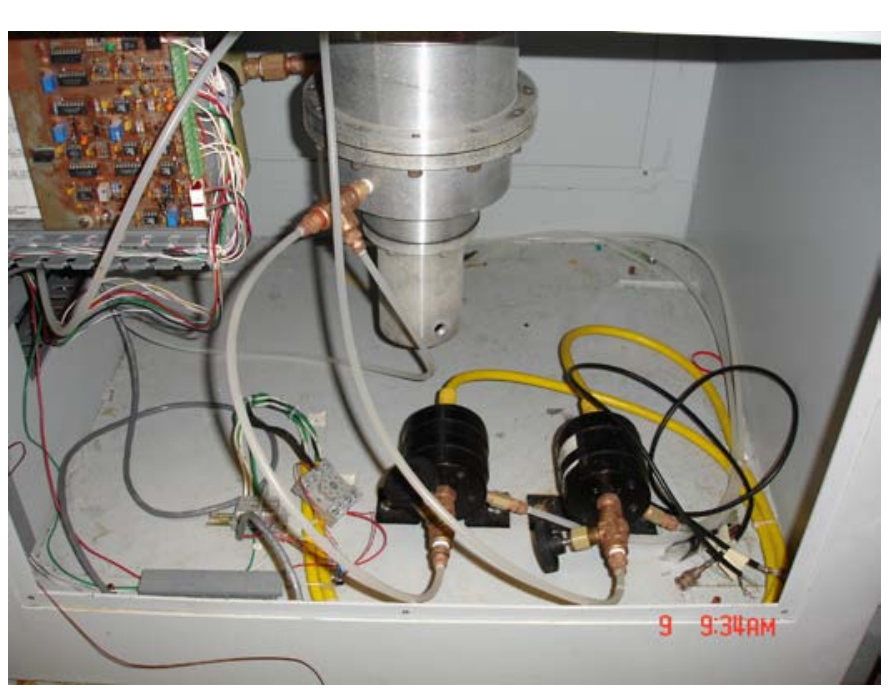

Fig. C13 ATS creep testing lower chamber that contains pneumatic pumps and system controller.

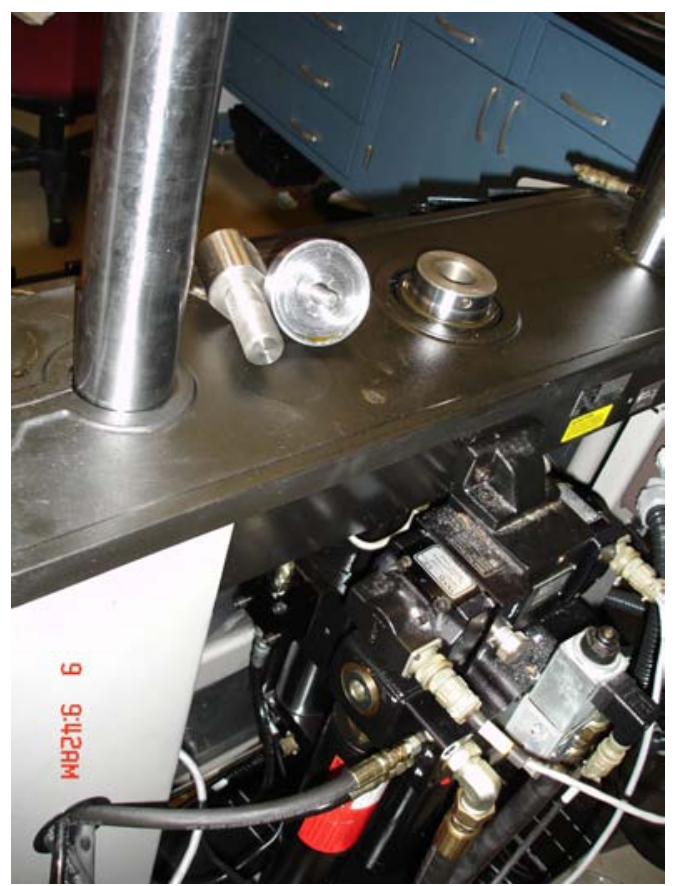

Fig. C14 The hydraulic oil leaking from actuator of MTS 810 tester. 


\section{C.3 Creep Testing Results for $1350-\mathrm{H} 19$ Aluminum}

The raw creep data carried out at the pneumatic ATS creep testers are illustrated below, where the laser mike is used for strain measurement.
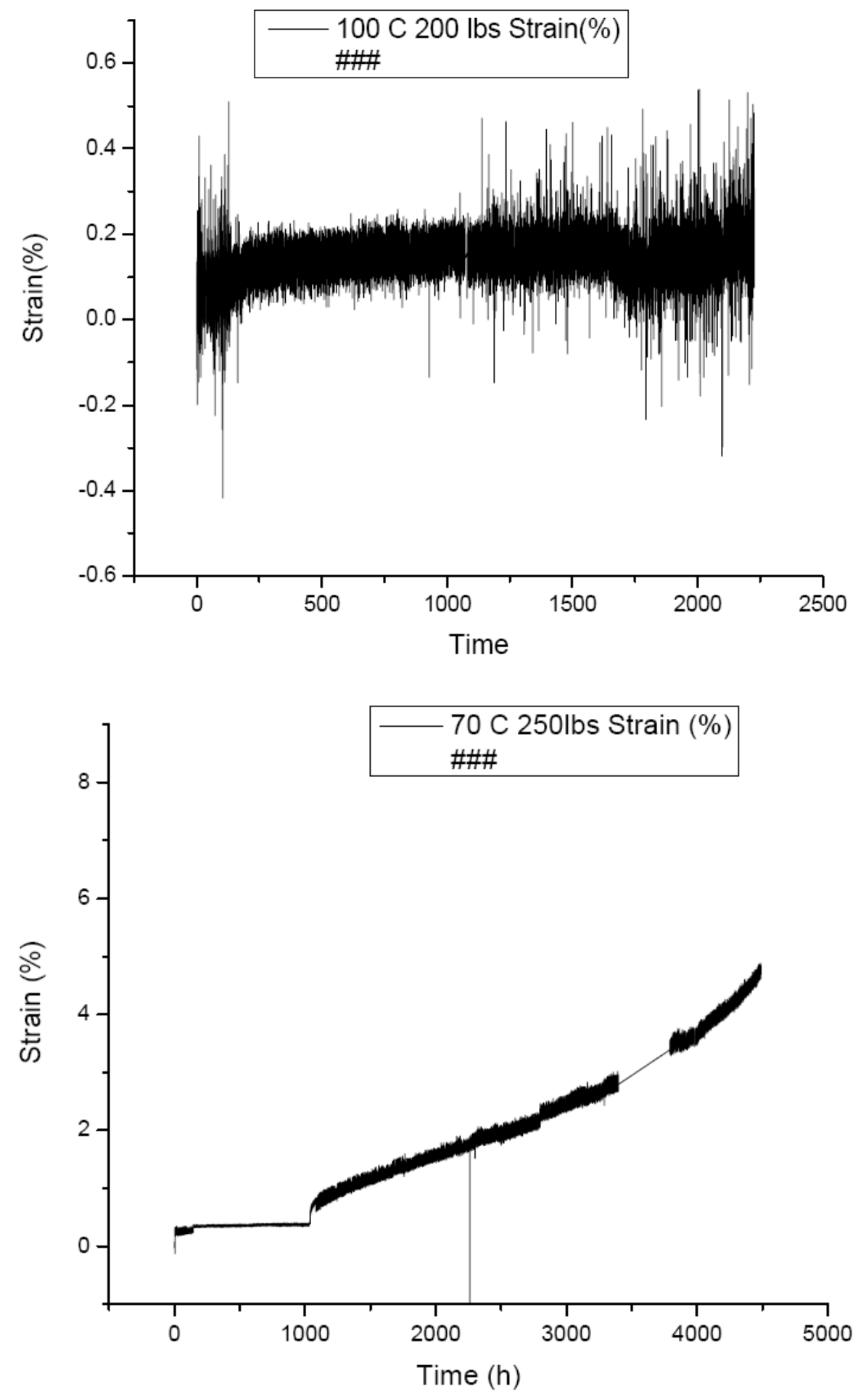

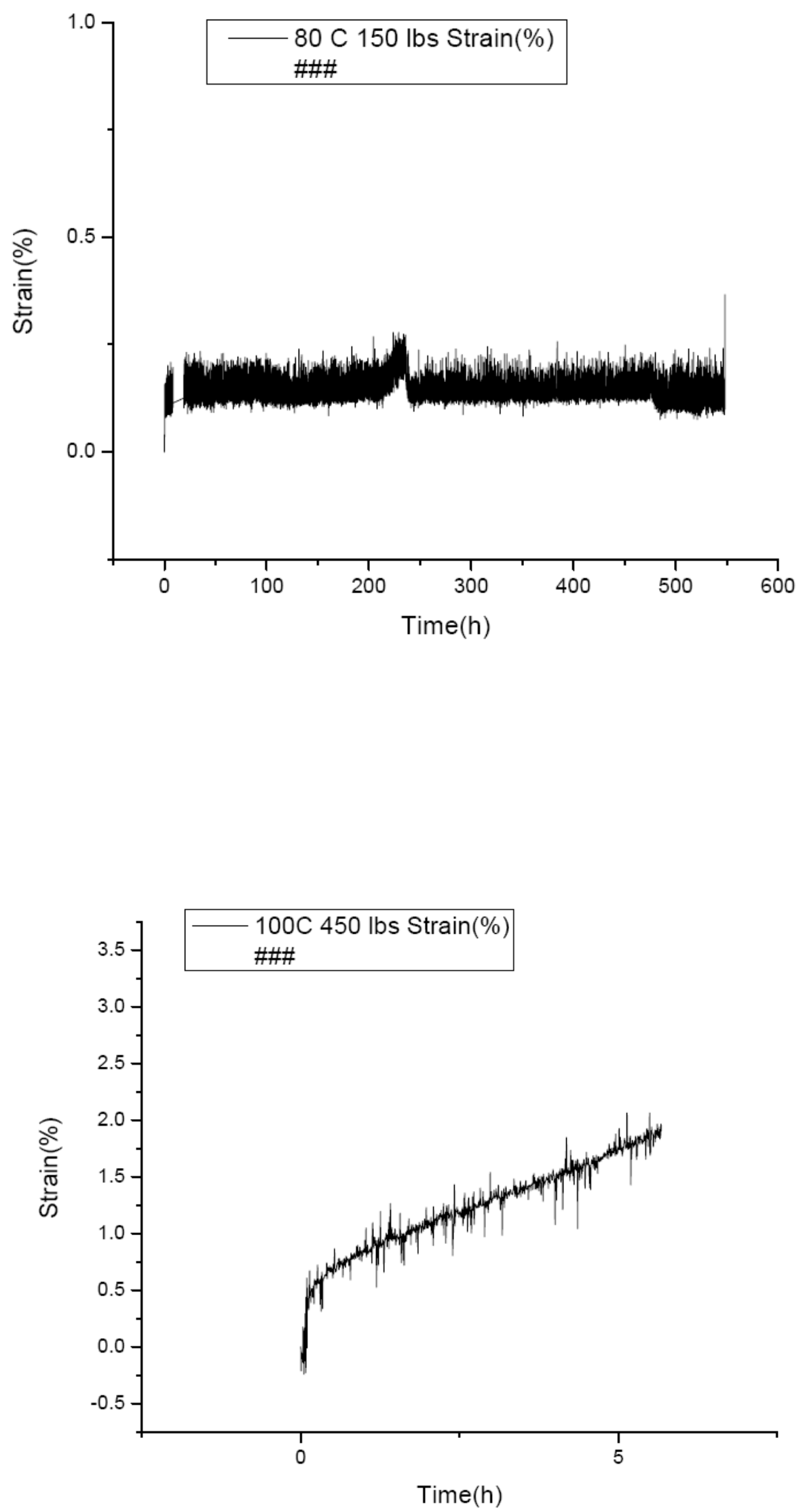

C-7 

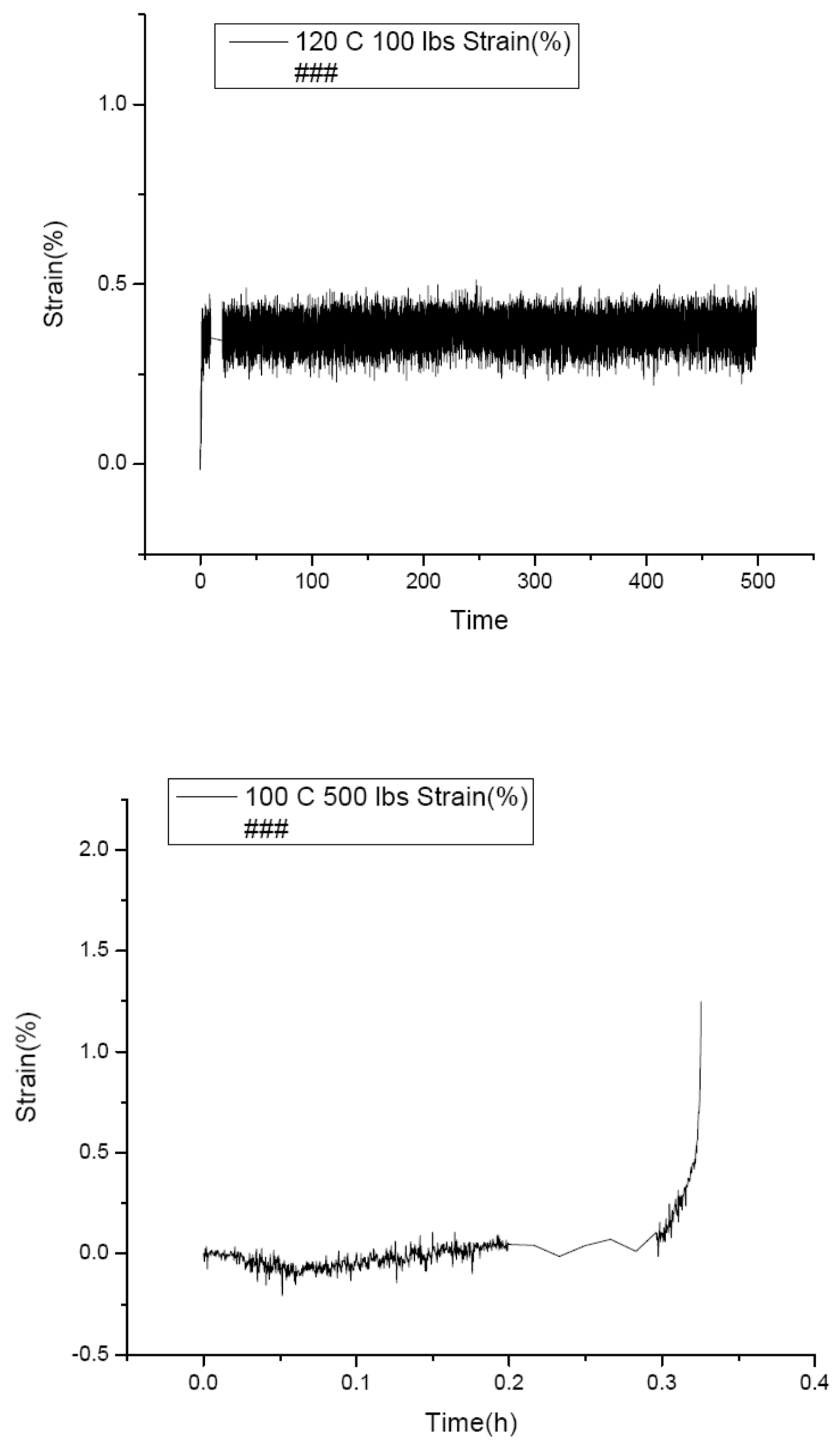

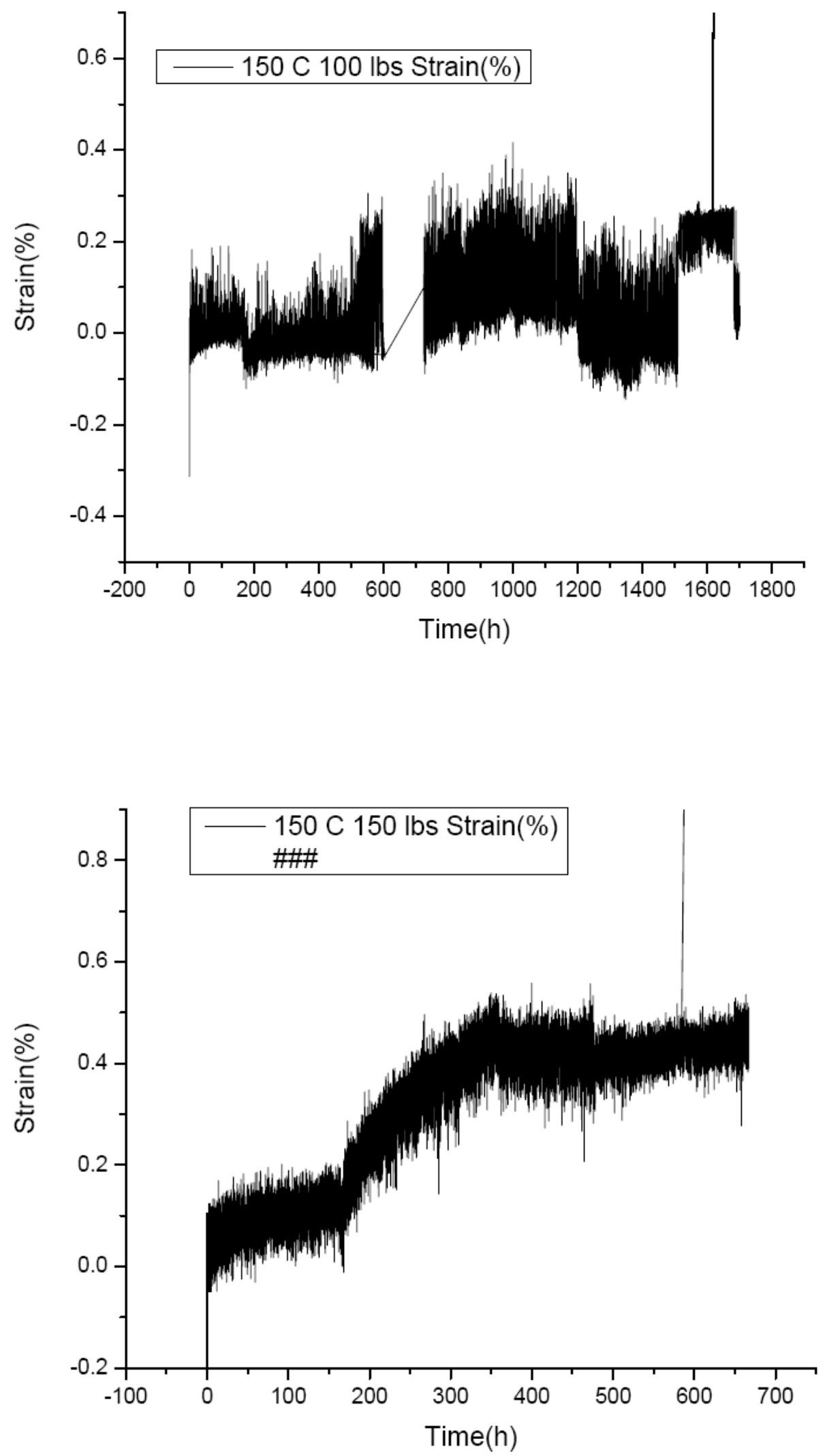

C-9 

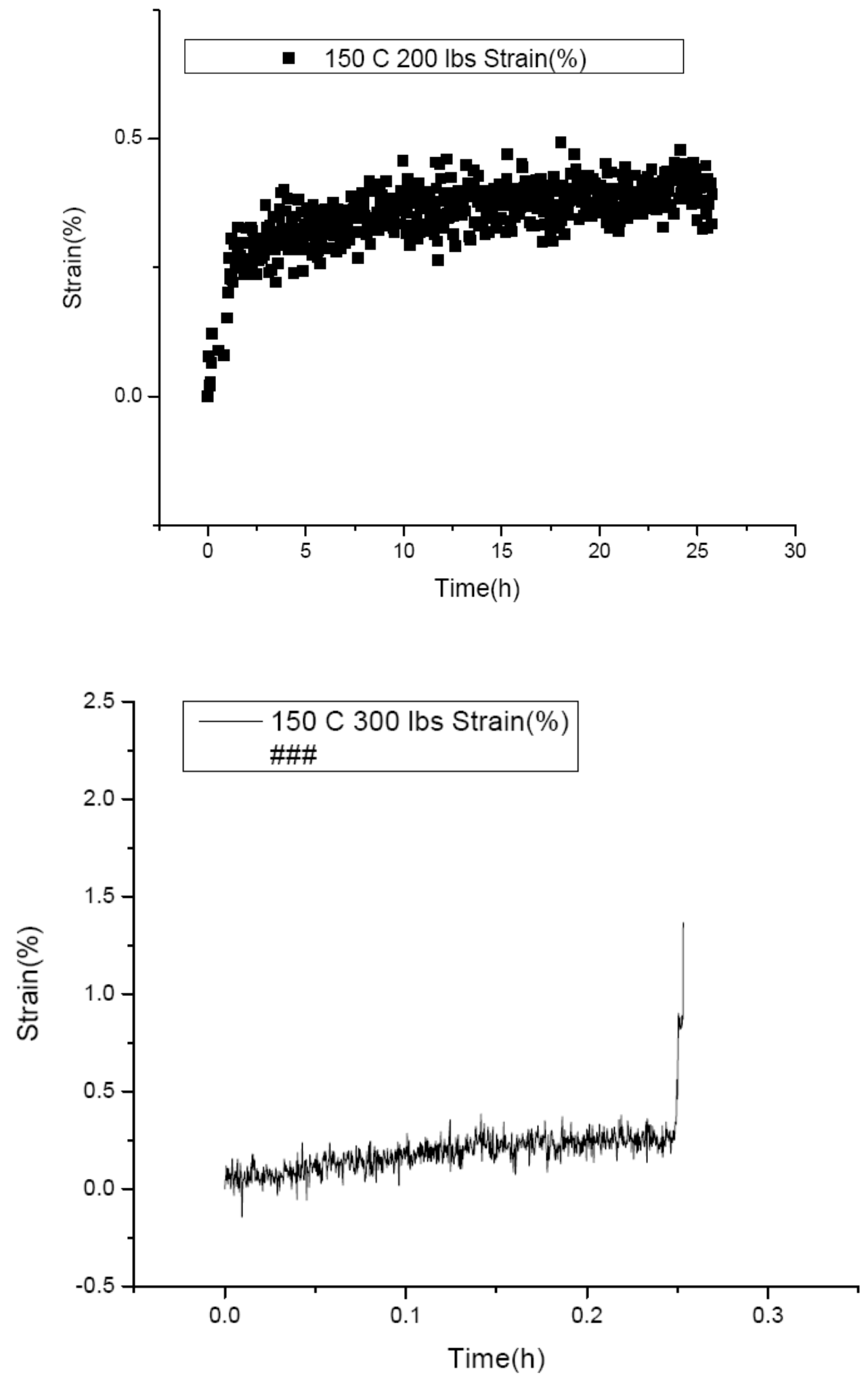

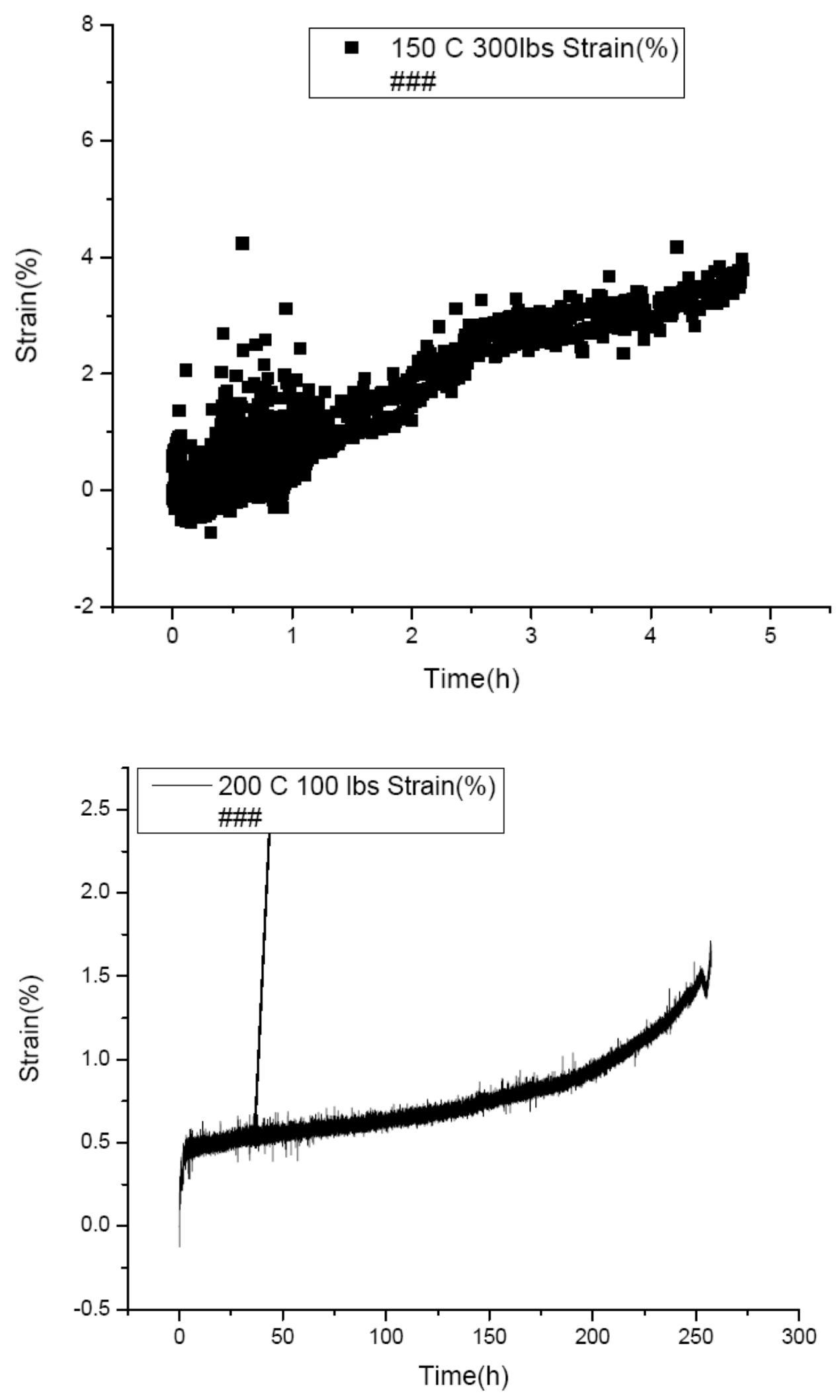

C-11 

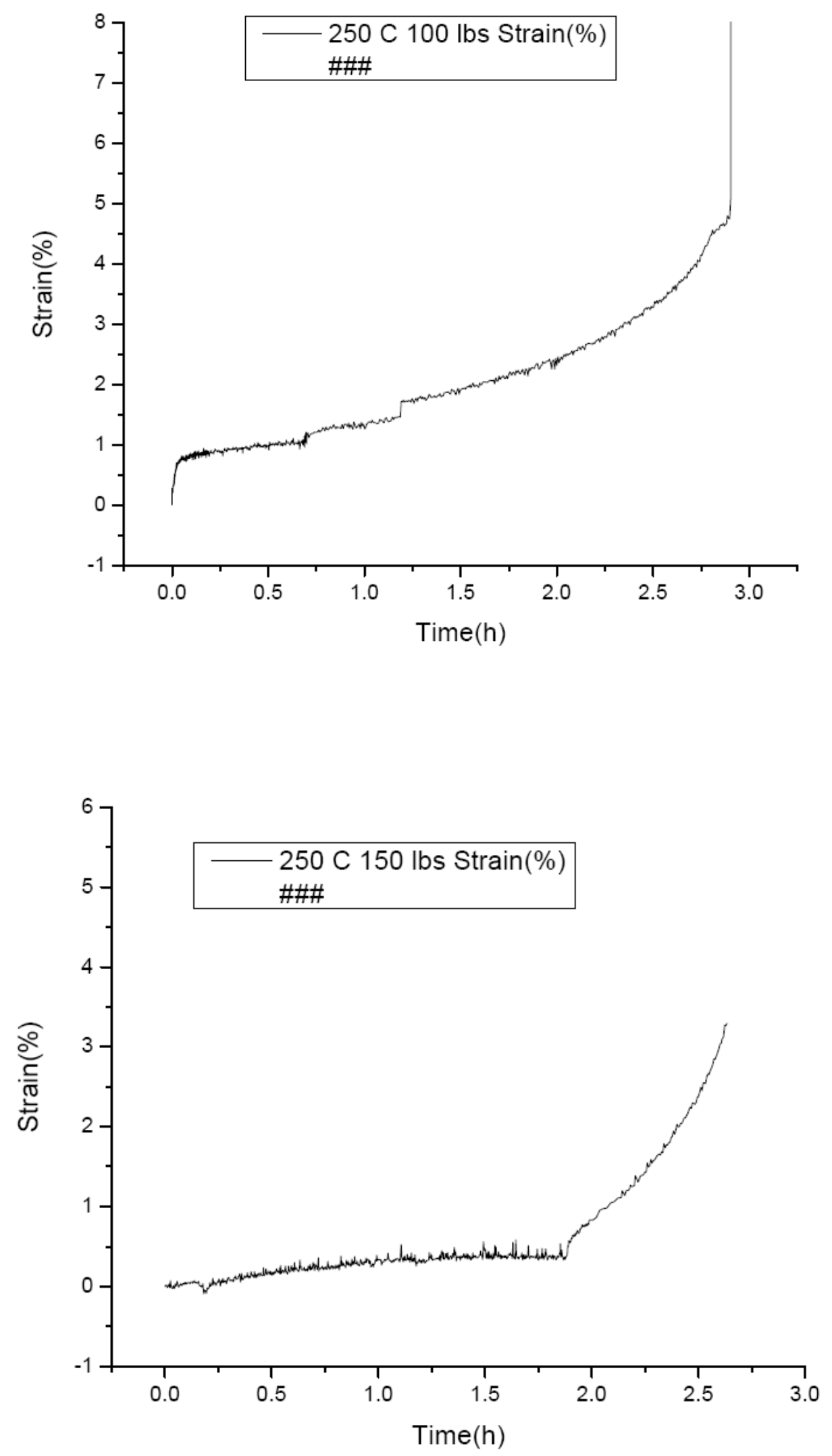


\section{C.2 Isothermal Fatigue Test Results}

Experimental data from fatigue experiments was analyzed using Microsoft Excel and Mathematica 5.2 software. Each fatigue data file contains information about the time, displacement, load, strain. The specimen diameter of 0.18 in was used in all experiments. The cross sectional area for each specimen was $16.417 \mathrm{~mm}^{2}$. The load in $\mathrm{N}$ when divided by this area gives stress in MPa at each data point.

In the data file A8, the strain readings from clip on gage were not reliable. Hence, laser mike readings were converted into the strain readings by $\left(\mathrm{d}_{\mathrm{i}}-\mathrm{d}_{0}\right) / 0.4904$.

For each data set, first the stress-strain loading cycles were identified in the initial, middle and end (closer to failure) parts of the experiment. Each data set may have several files pertaining to the same specimen subjected to loading fatigue cycles.

Mathematica software was used to partition the data sets into cycles and then figure out the maximum stress and strain in each cycle. The output files produced contain cycle number, peak stress and peak strain for each cycle. The plots of stress and strain as a function of cycles will reveal softening or hardening behavior of the material. It will also provide failure stress and number of cycles to failure, for the fixed peak strain.

The following pages show some of the plots produced using this procedure:

\section{Data set a14}

These data pertain to $6061-\mathrm{T} 6$ material subject to fatigue testing at $205^{\circ} \mathrm{C}$. Each cycle had a strain range maintained at $\pm 0.2 \%$. 


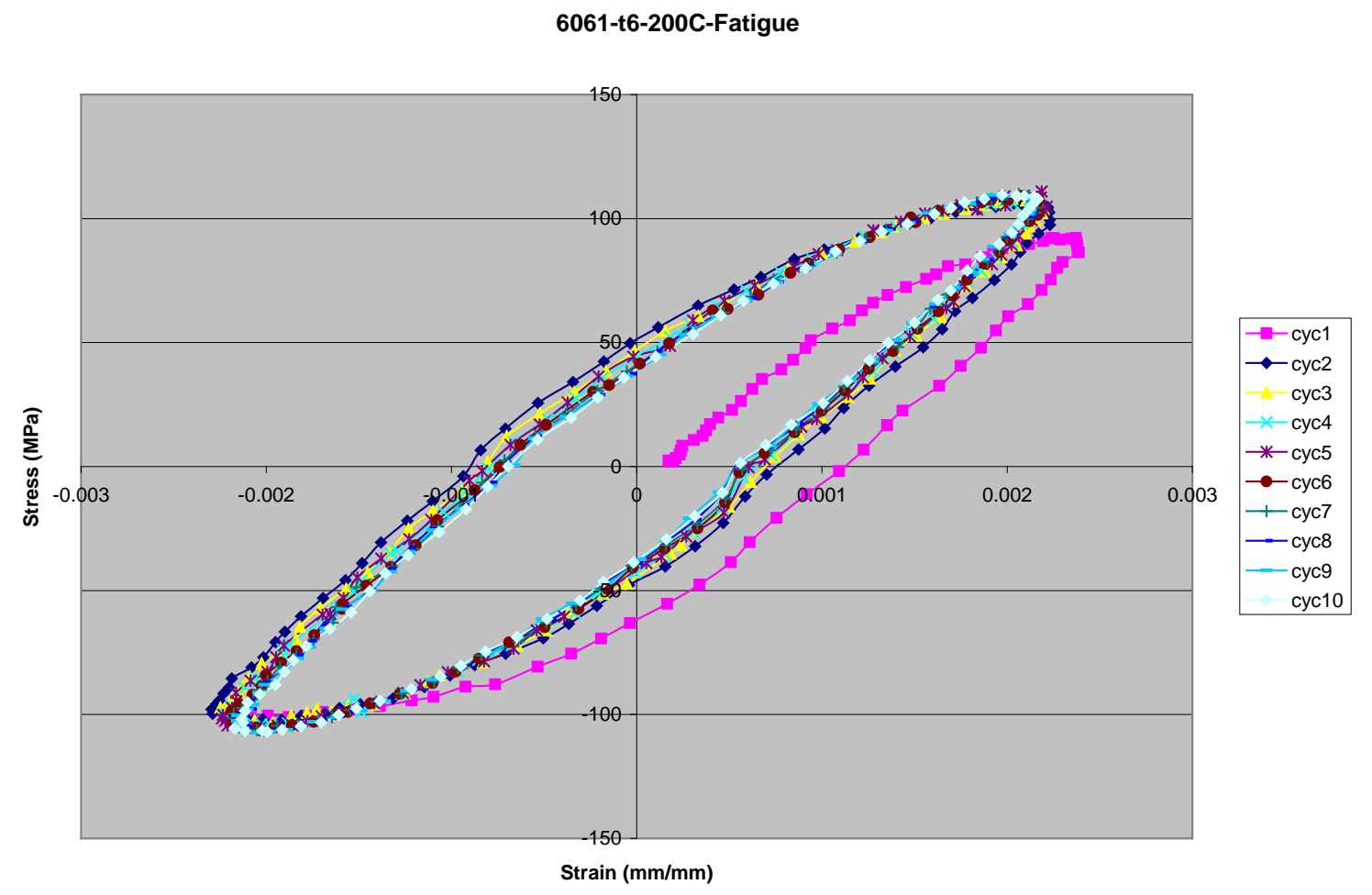

Gradual stiffening (hardening) is evident in initial loading cycles. The peak stress increases while the strain range is identical.

6061-T6-A14-200C-Fatigue

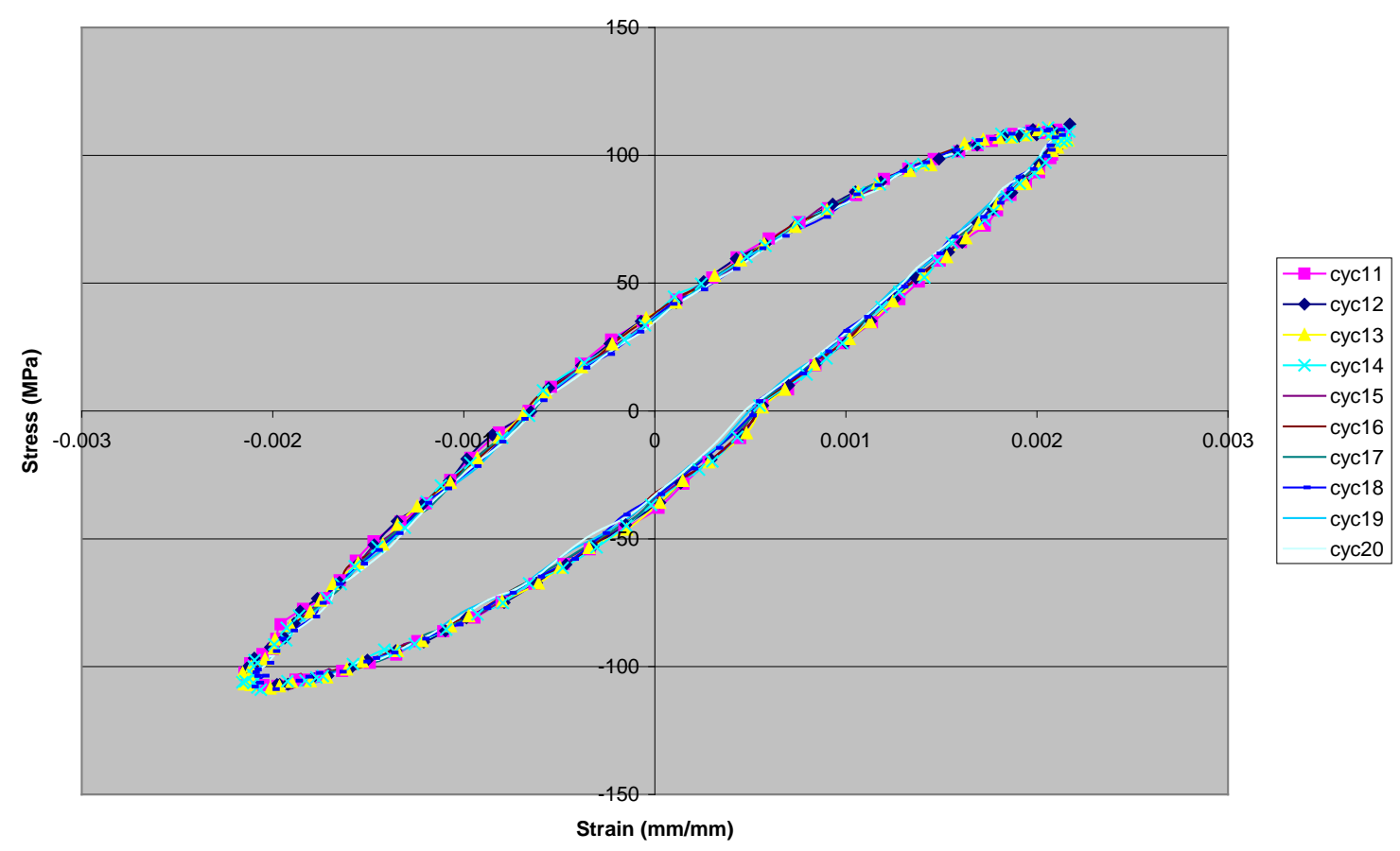




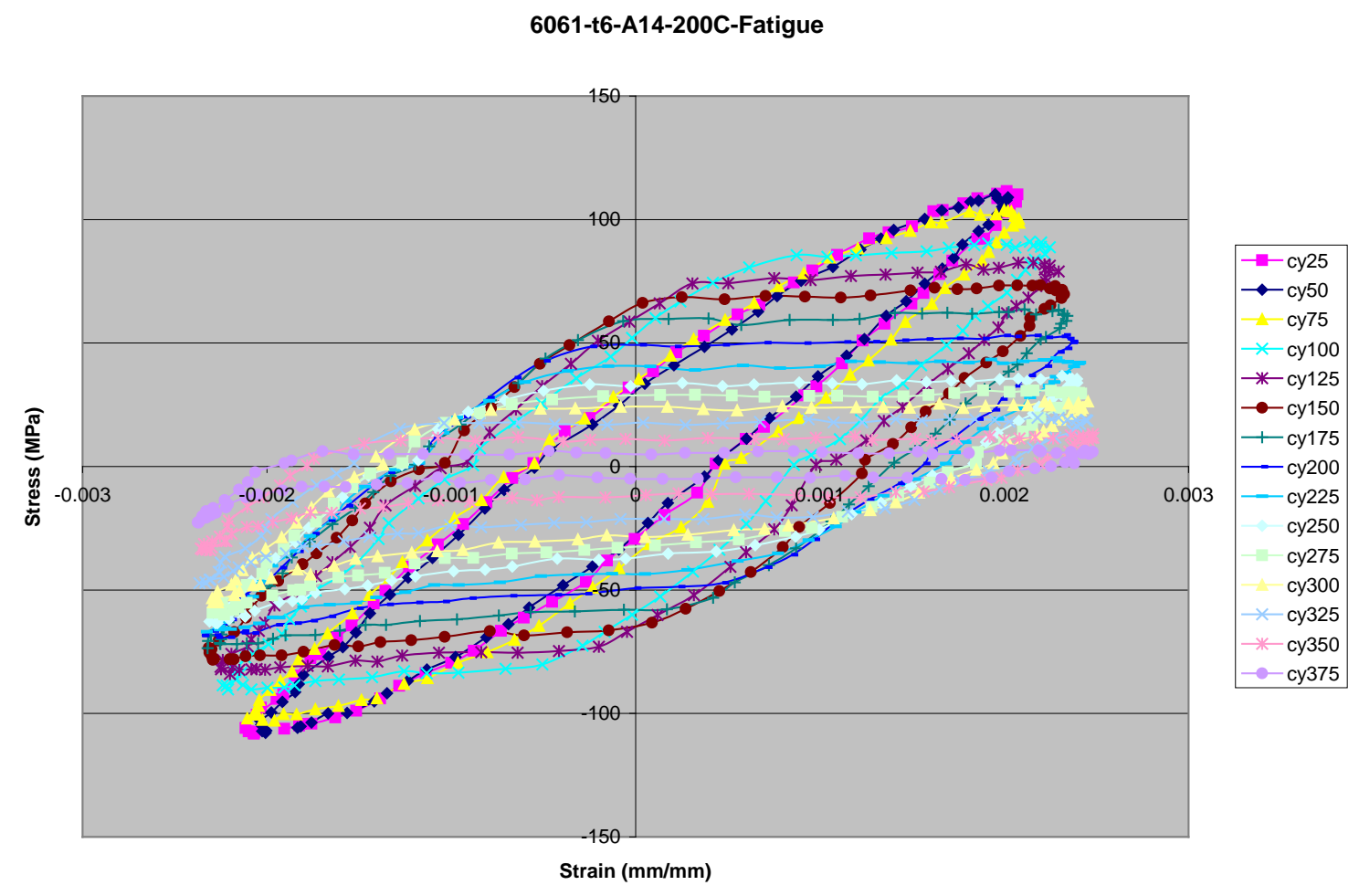

The softening of material in tension and compression is identical. The material shows pronounced softening, yielding and permanent deformation after about 150 cycles. For all practical purposes, material fails to carry stress after about 350 cycles.

Peak Stress Vs Cycles

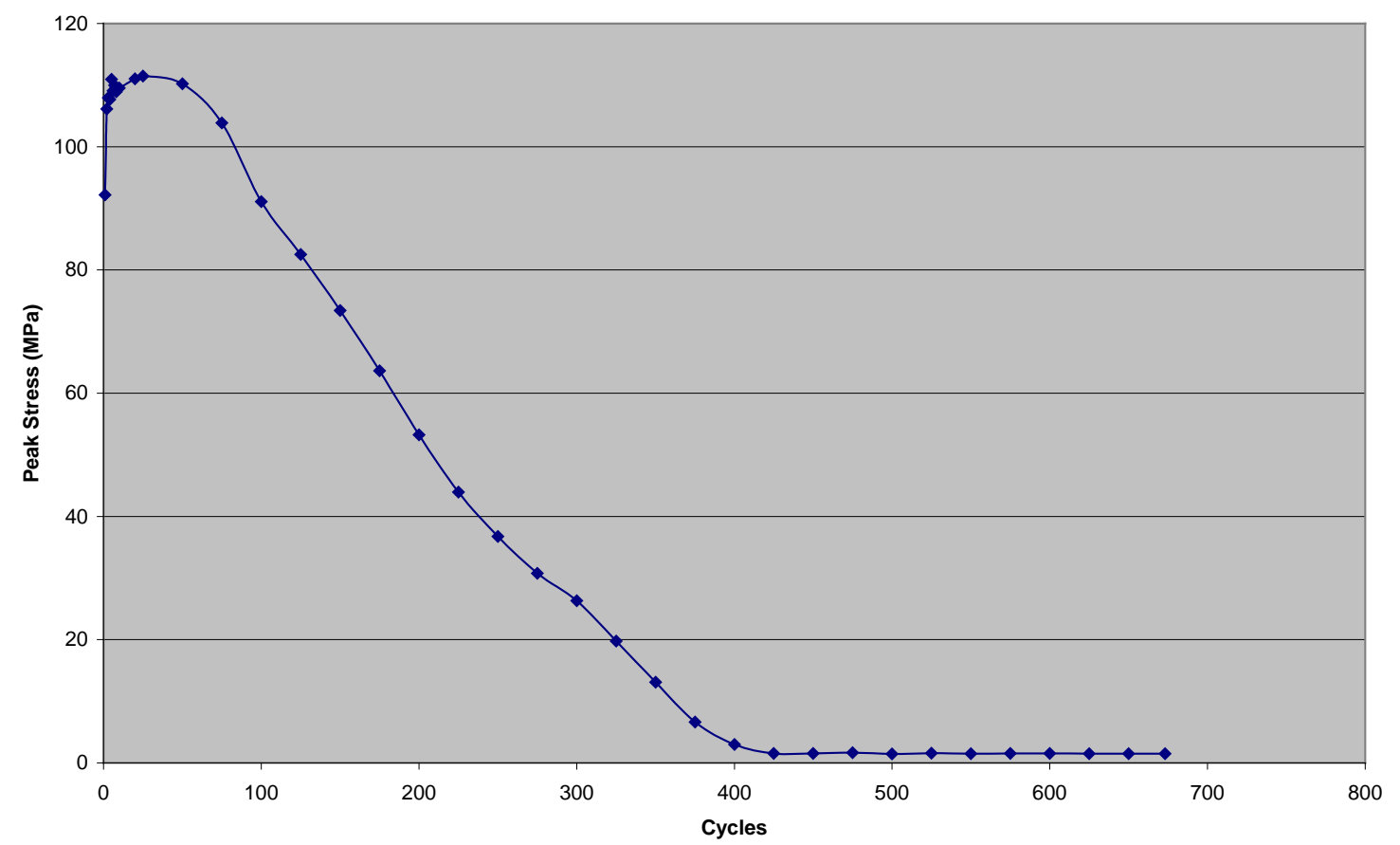


The peak stress plot shows initial strain hardening followed by prolonged gradual softening and eventual failure when subject to fatigue cycles.

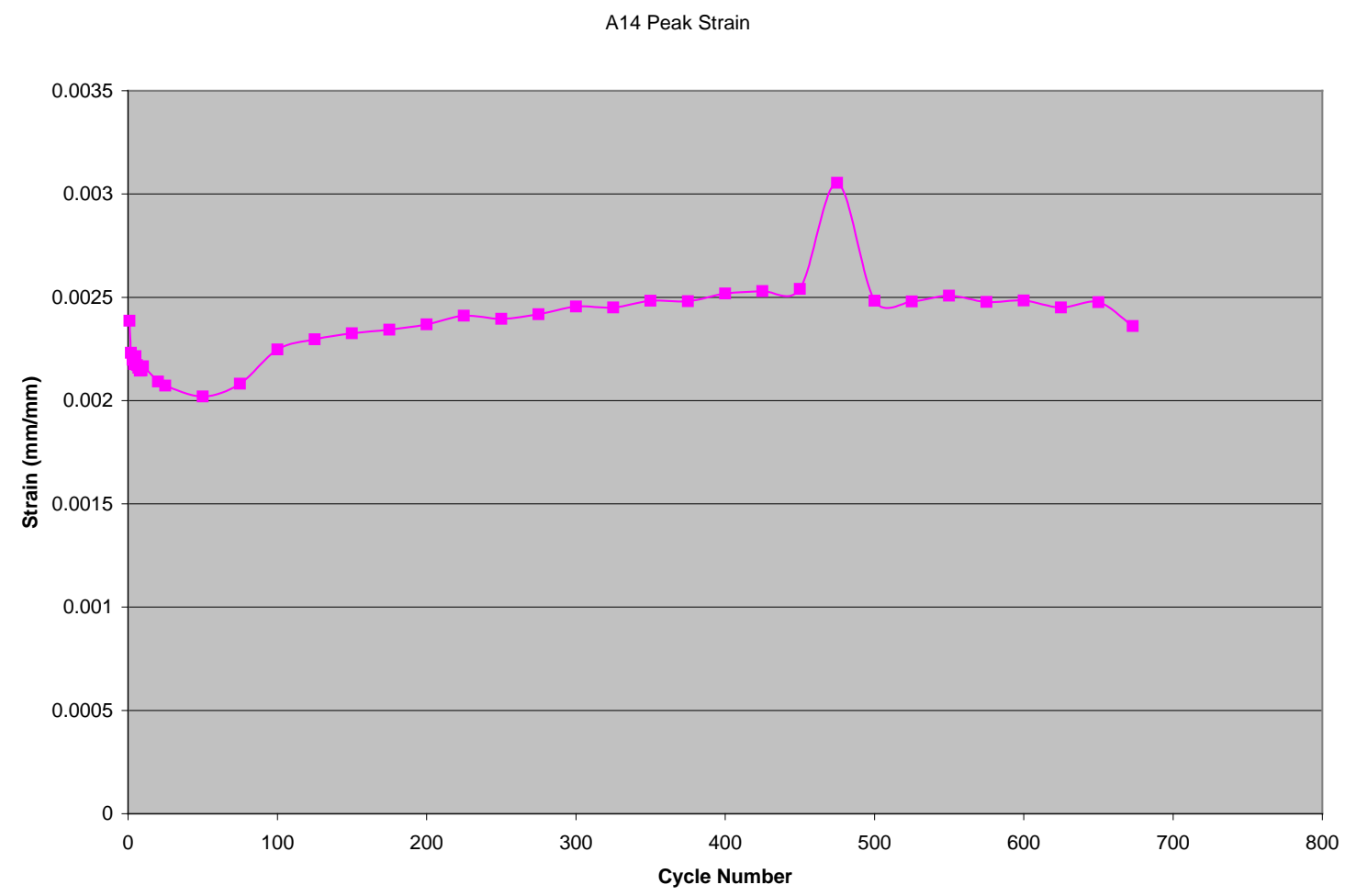




\section{File Data Set A10:}

These data pertain to $1350-\mathrm{H} 19$ material tested at room temperature. The strain range was maintained at $\pm 0.2 \%$.

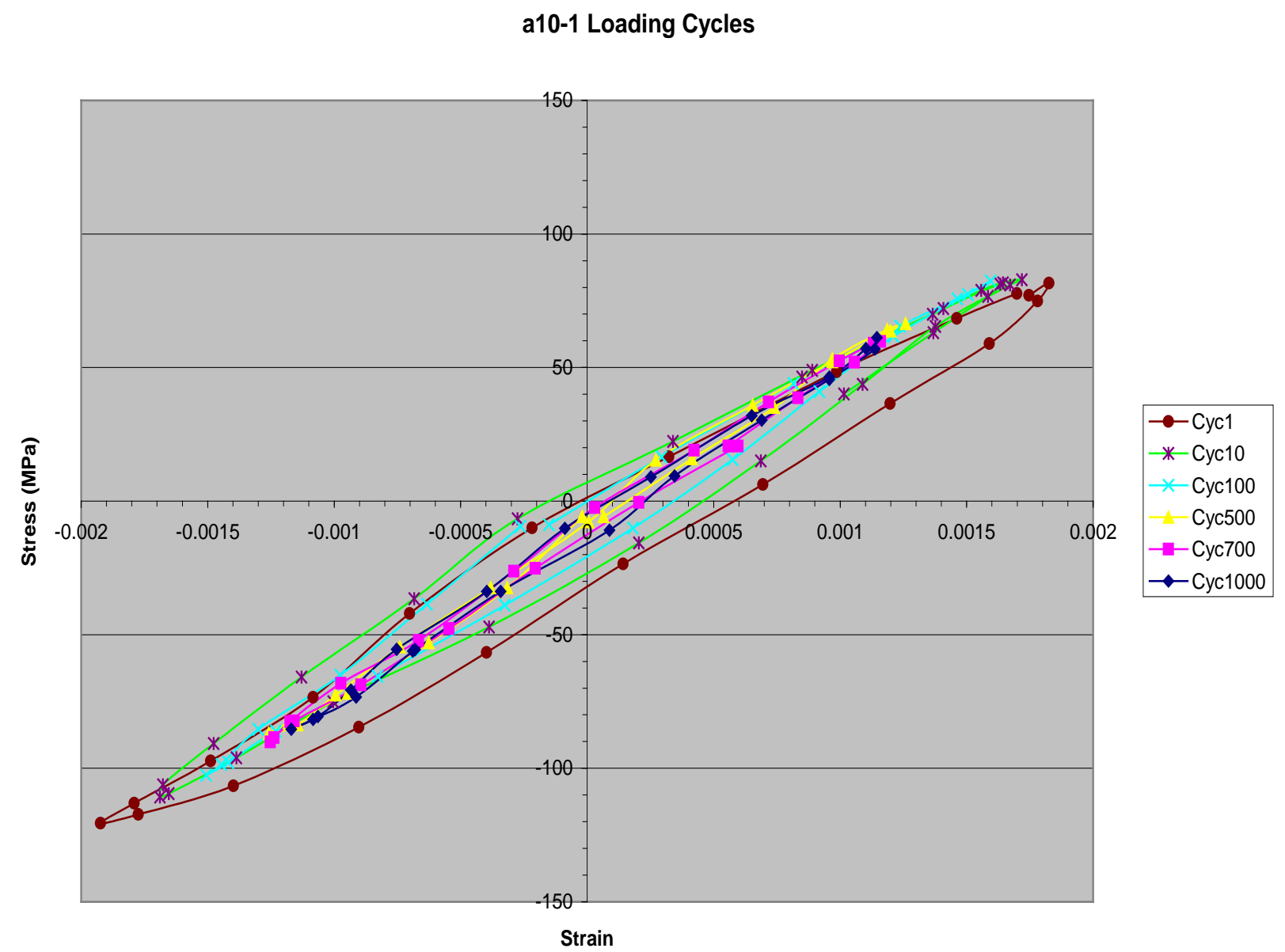


a10-1 Max Cycle Stress

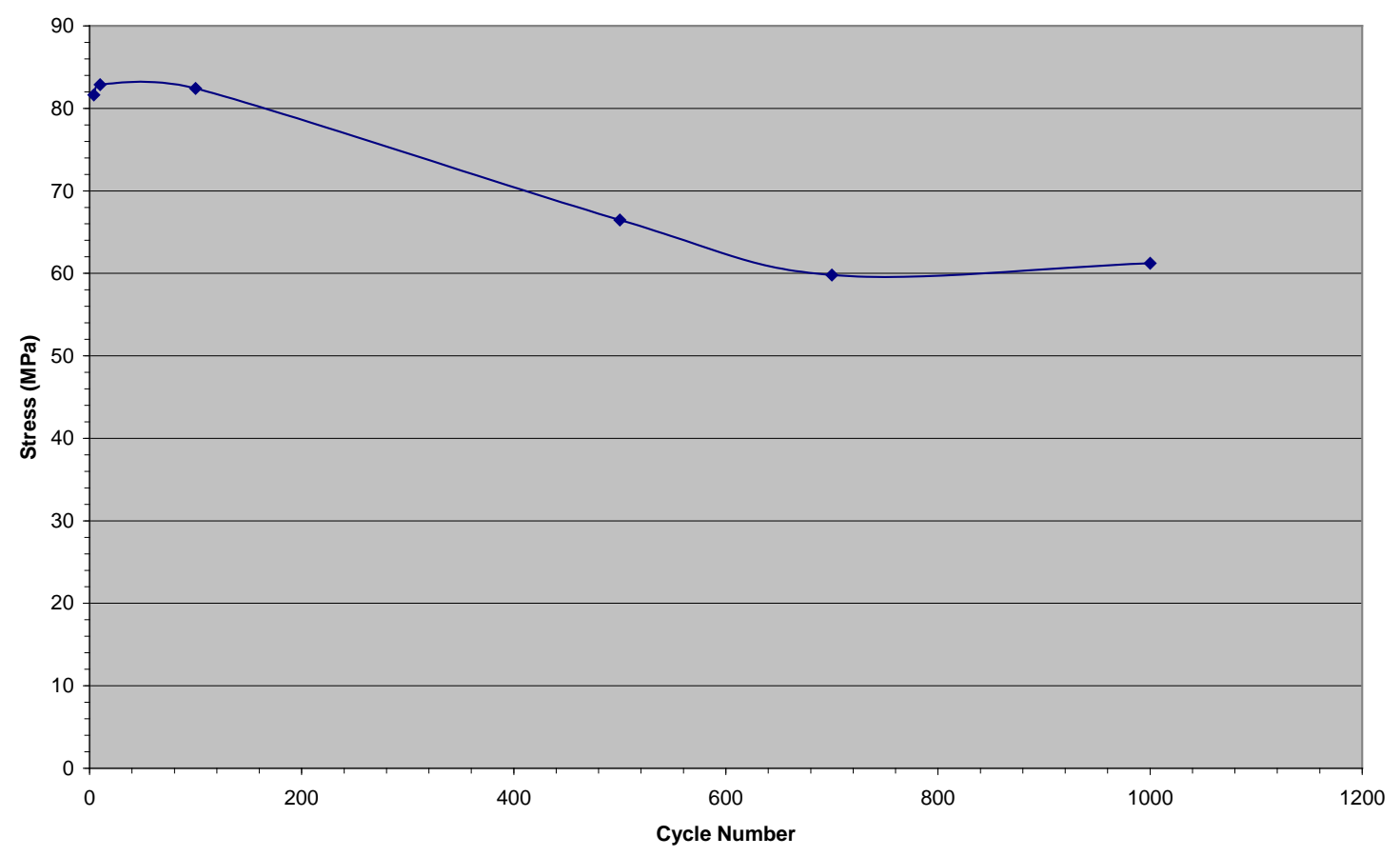

The material exhibits gradual softening up to about 700 cycles, beyond that it probably stabilizes to its endurance stress limit for $0.2 \%$ strain.

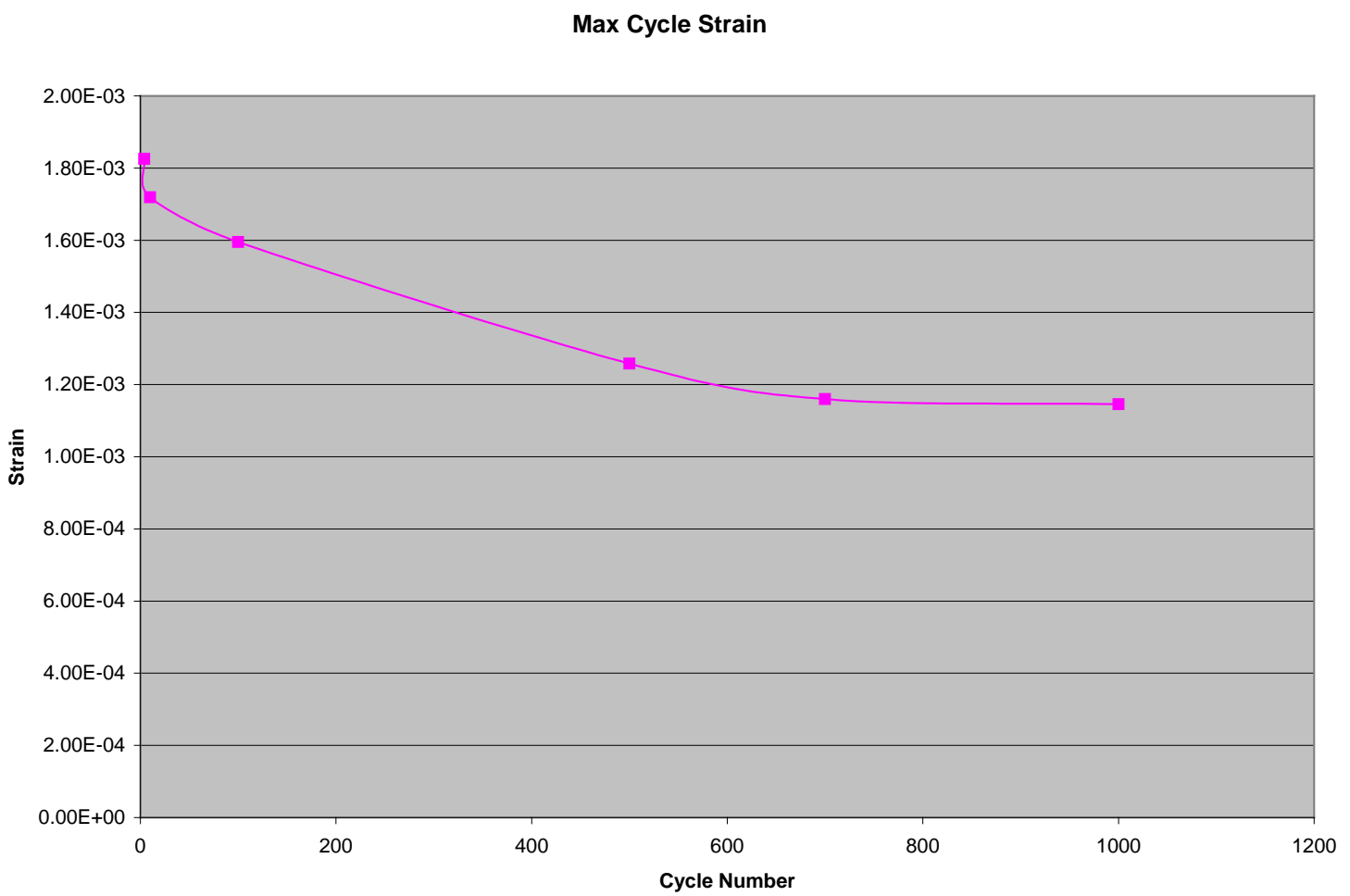




\section{File Data Set A9:}

These data pertain to 1350-H19 material subjected to cyclic loading at room temperature.

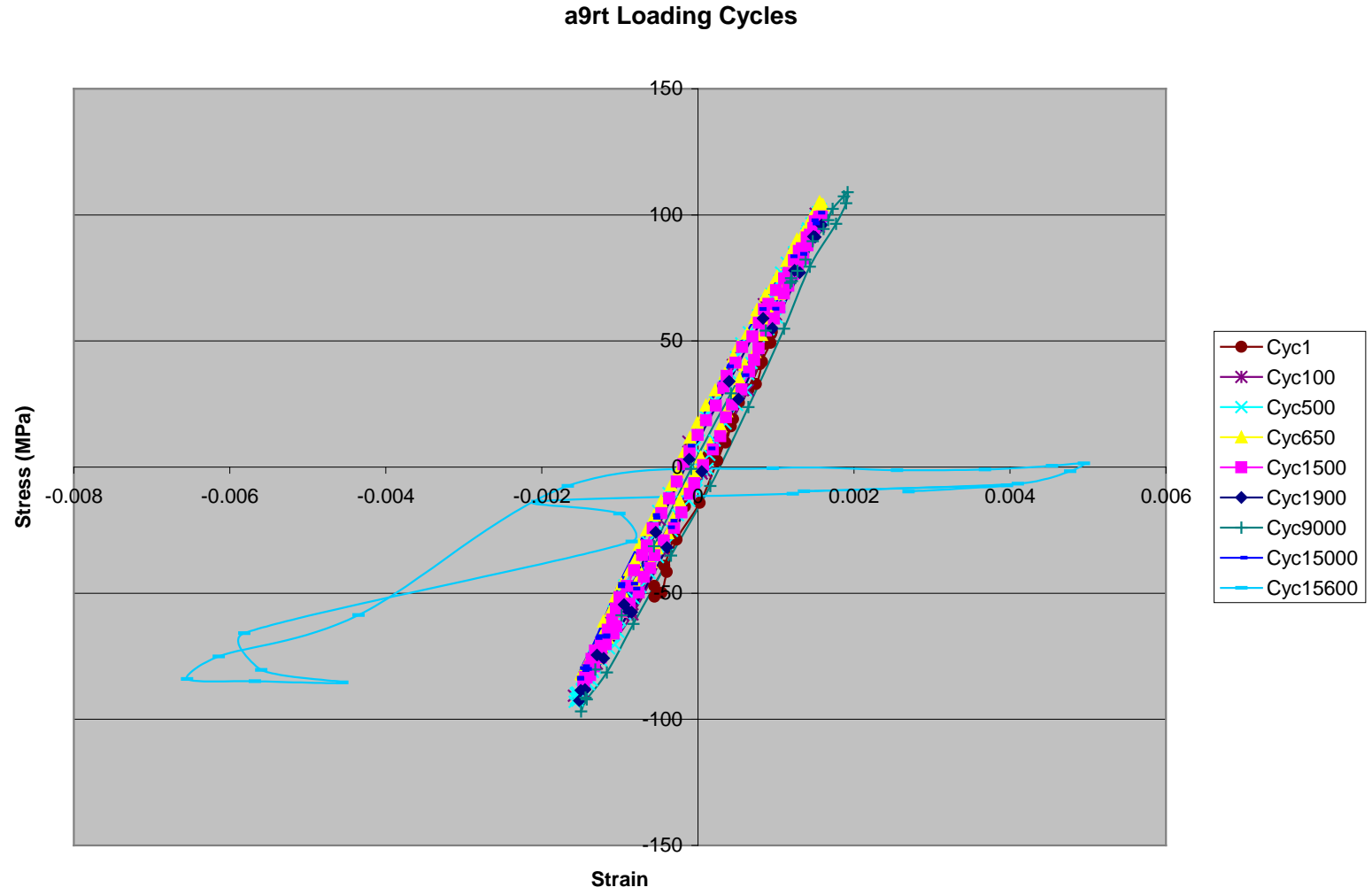

a9-rt Max Cycle Stress

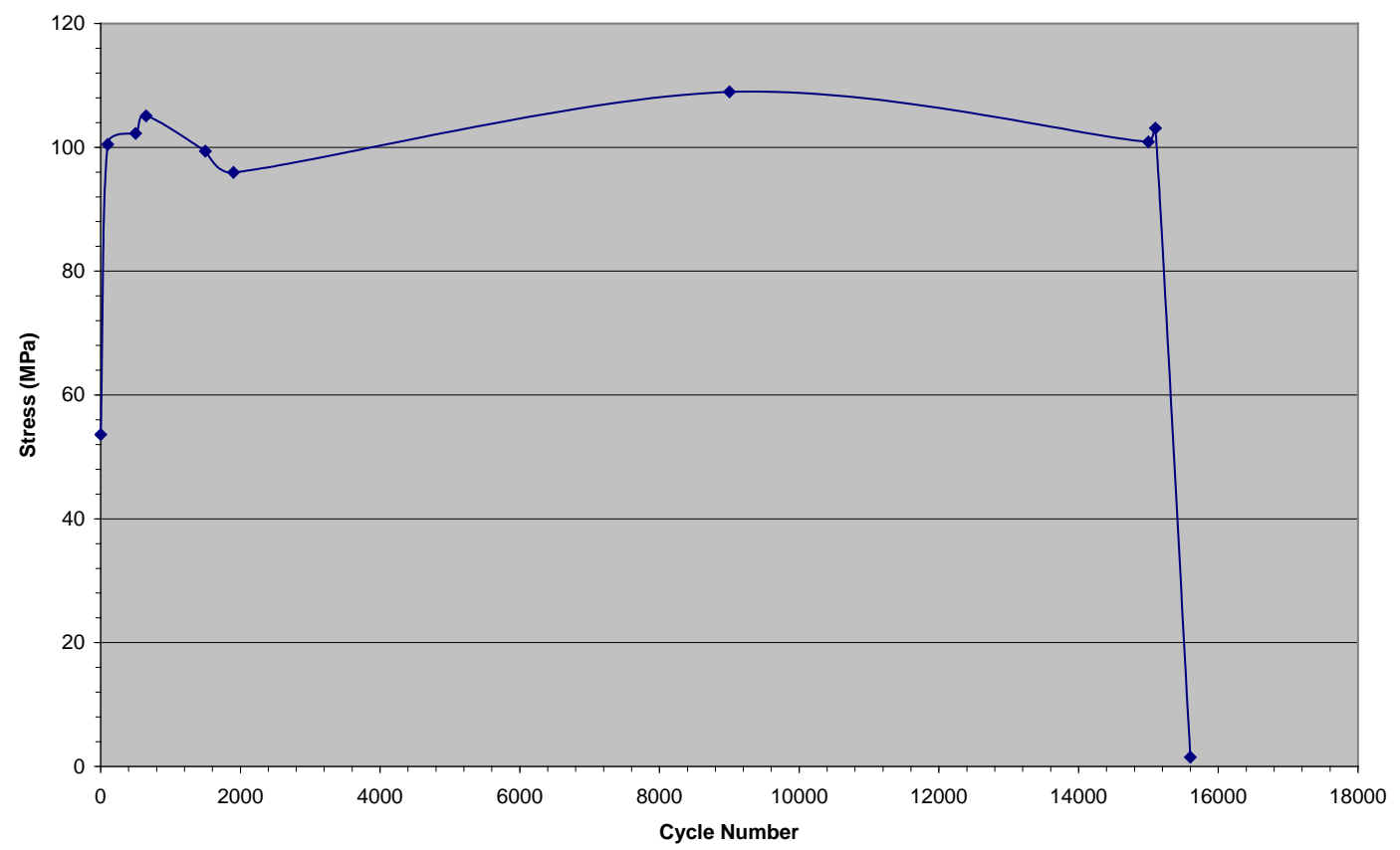


a9-rt Max Strain

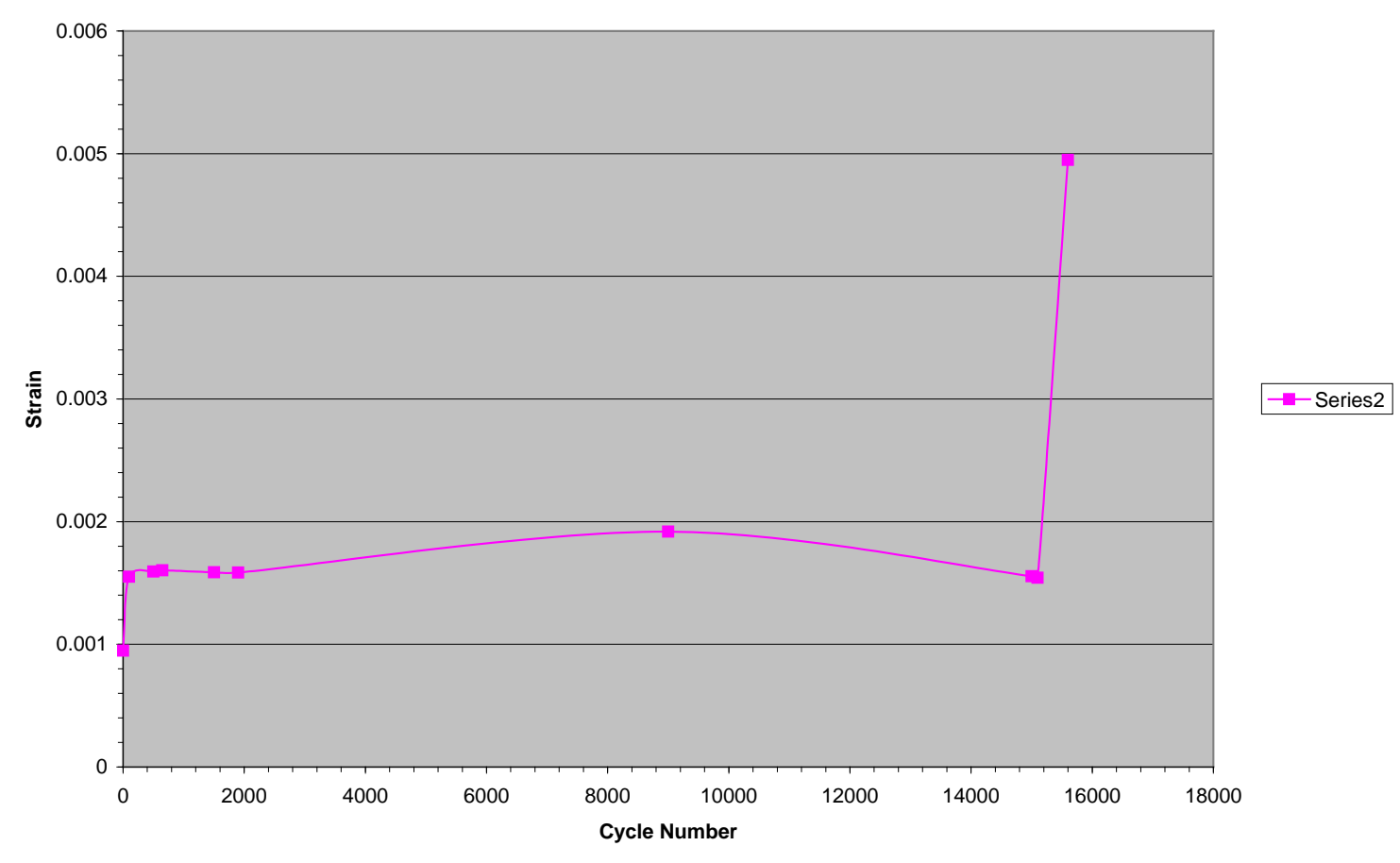




\section{B12-isot-23}

These data pertain to $3003-\mathrm{H} 183$ at $456{ }^{\circ} \mathrm{C}$ subject to cyclic loading limited by strain range of $\pm 0.3 \%$.

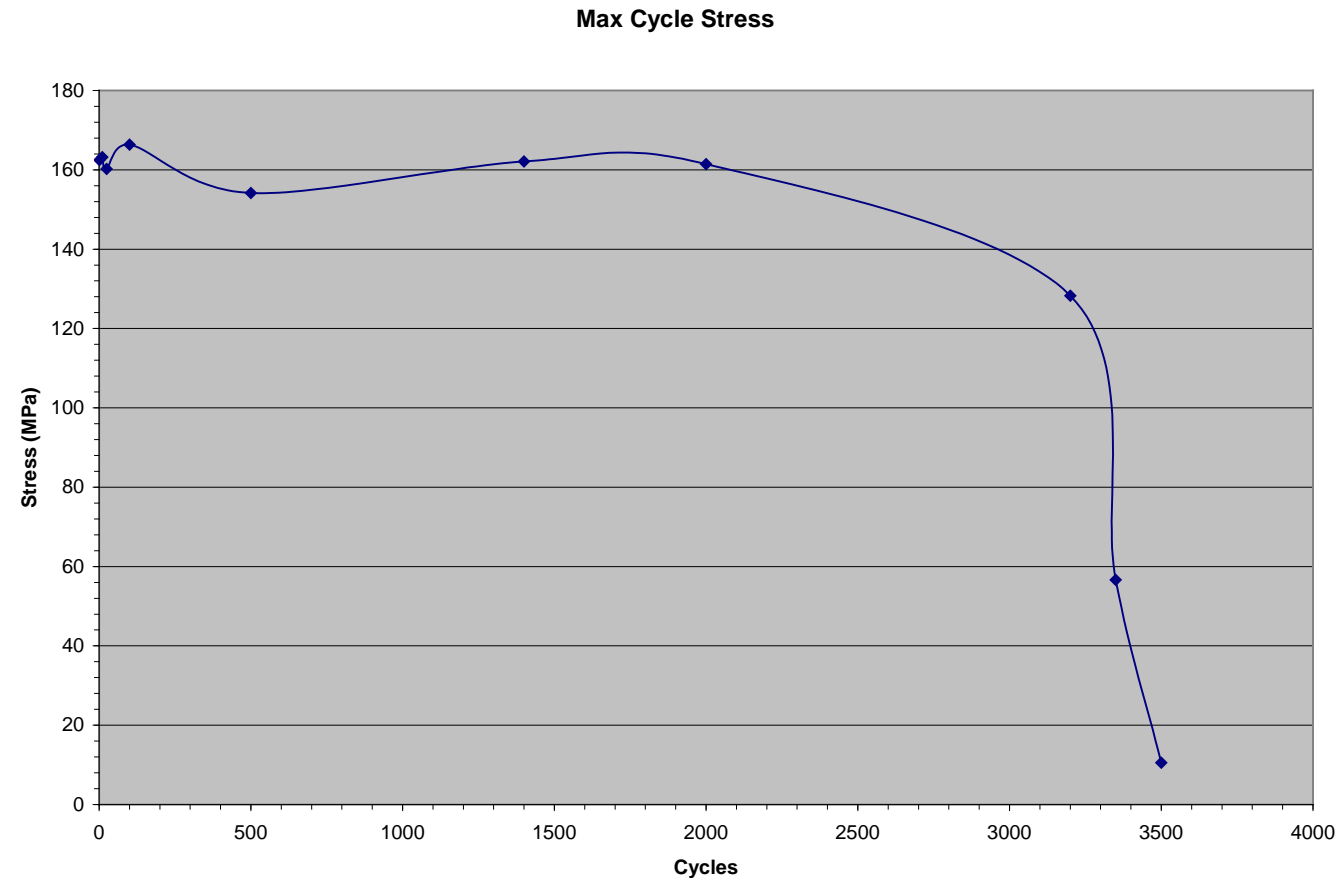

B12 Cycles

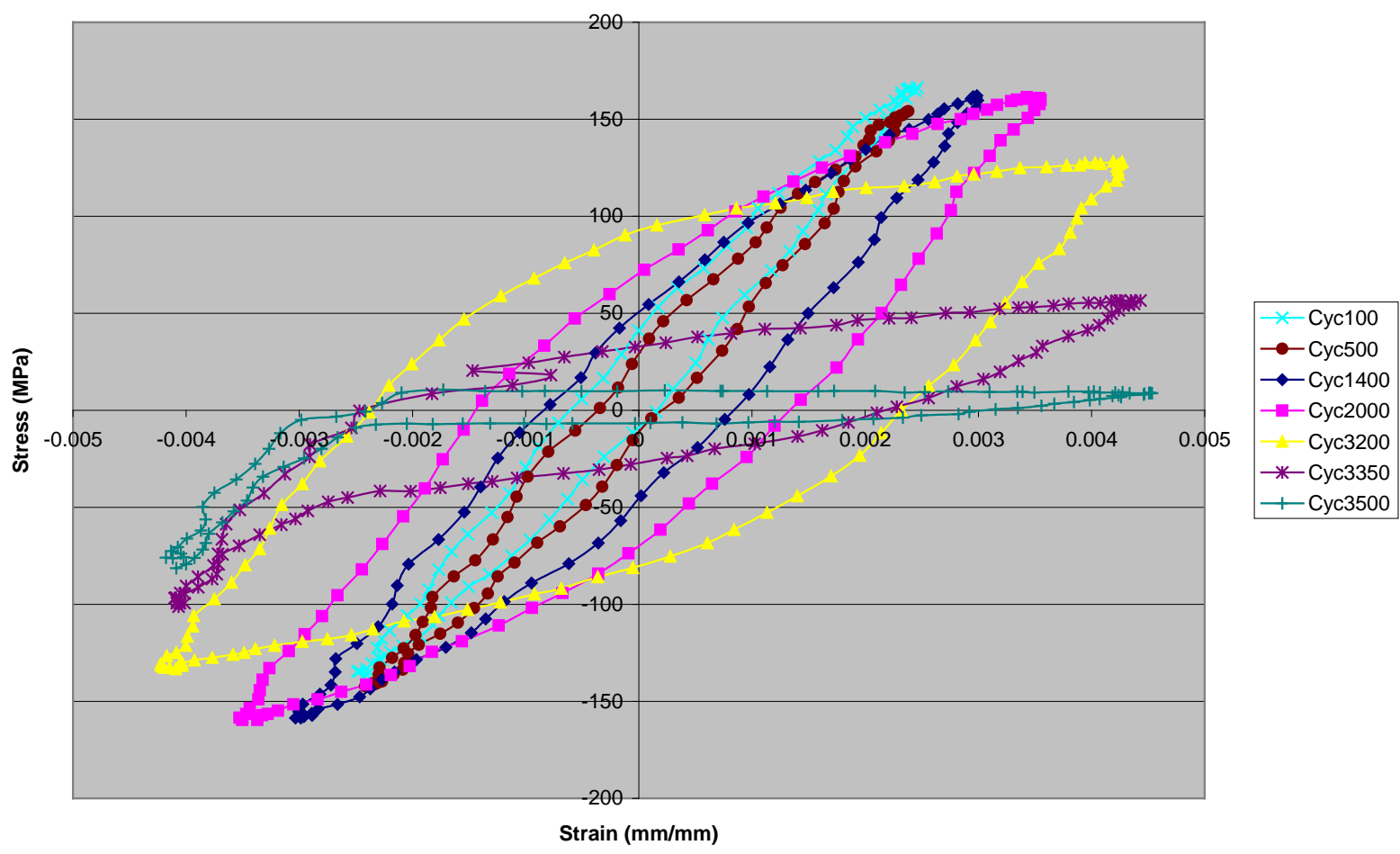

C-21 


\section{B10-RT}

These data pertain to 3003-H183 subject to cyclic loading at room temperature. 
B-10-RT Max Cycle Stress

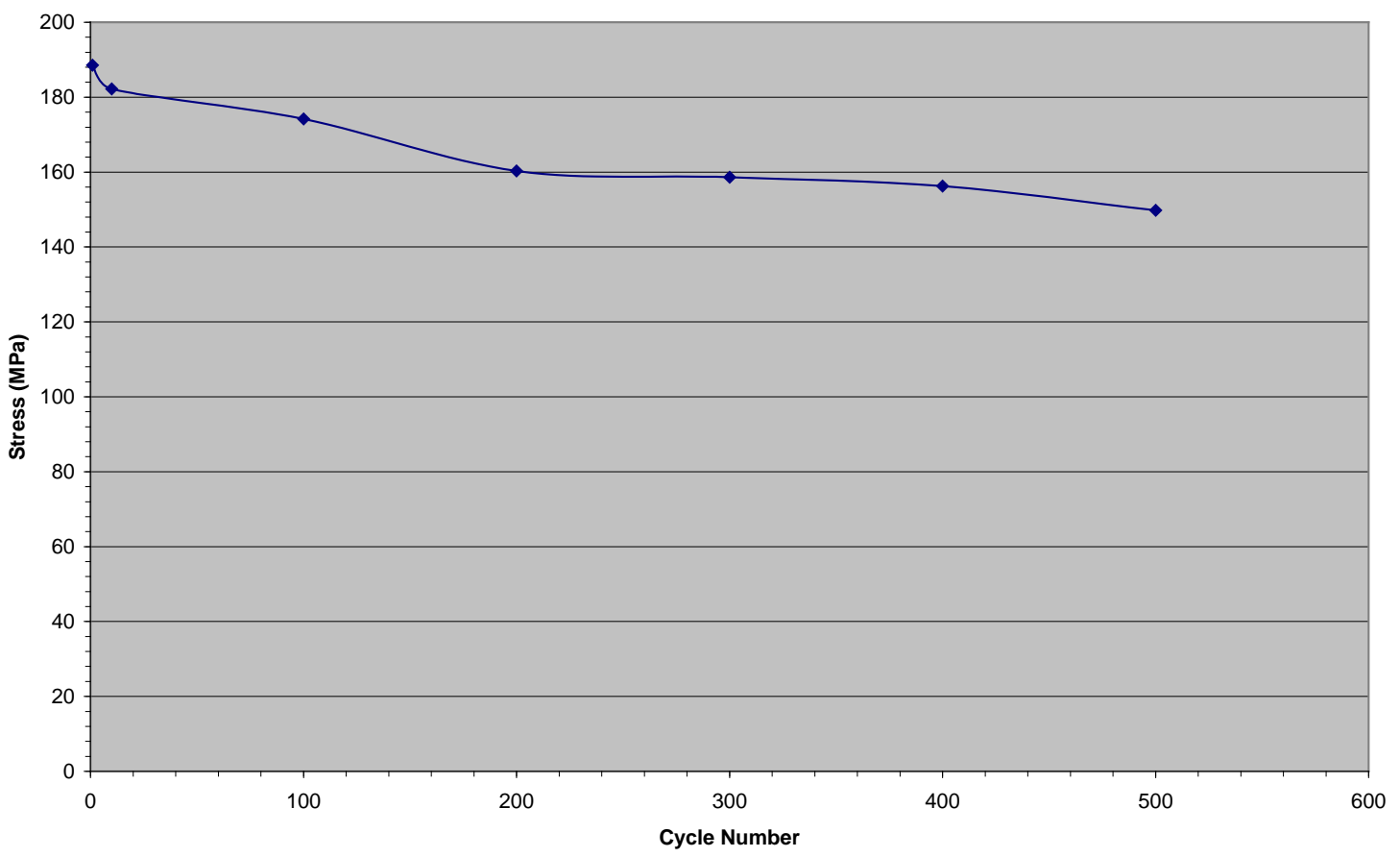

B10-RT Max Cycle Strain

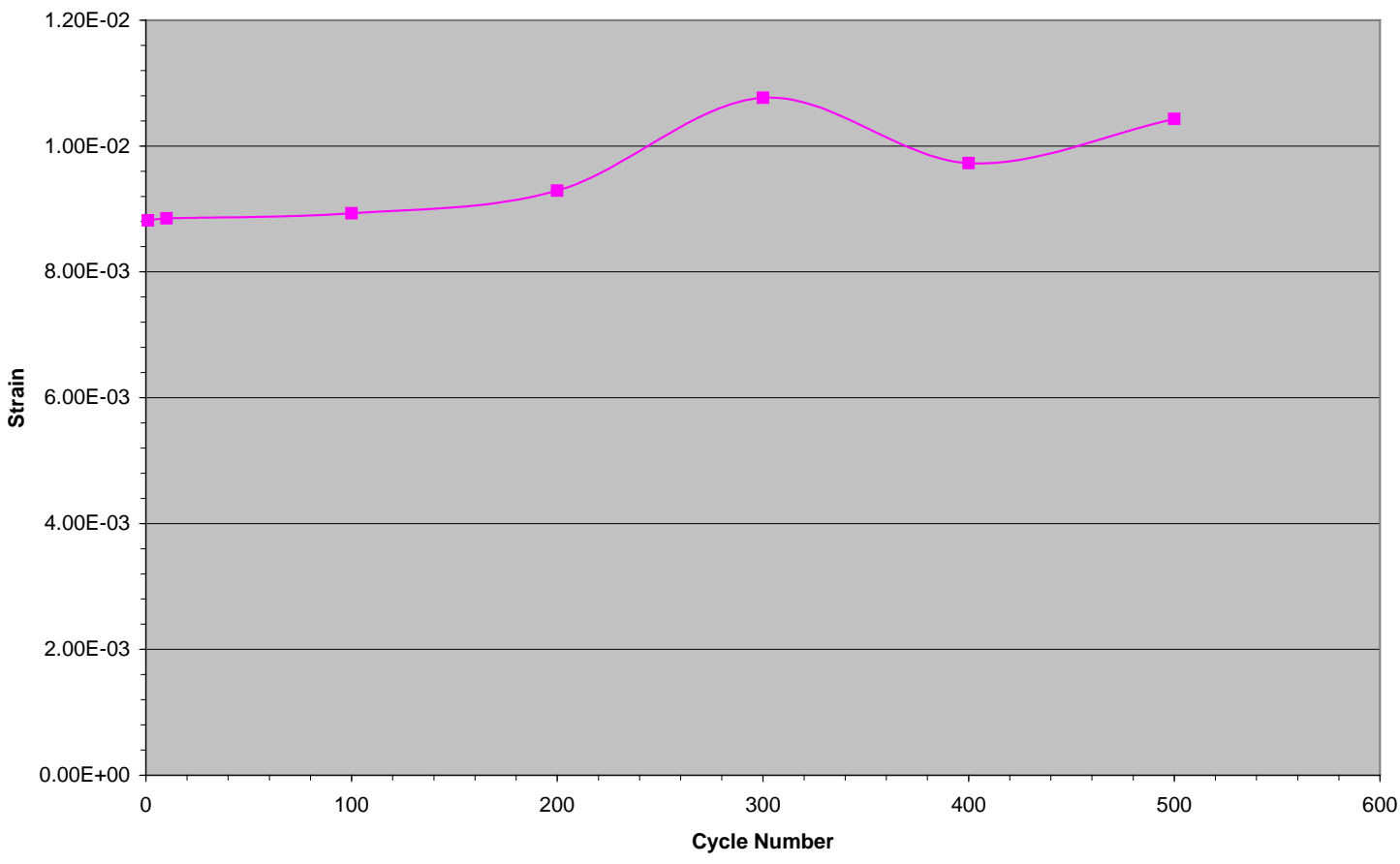




\section{Appendix B}

\section{A22-isot-200}

These data pertain to $1350-\mathrm{H} 19$ material at 200 degree C subject to cyclic loading.

Max Cycle Stress

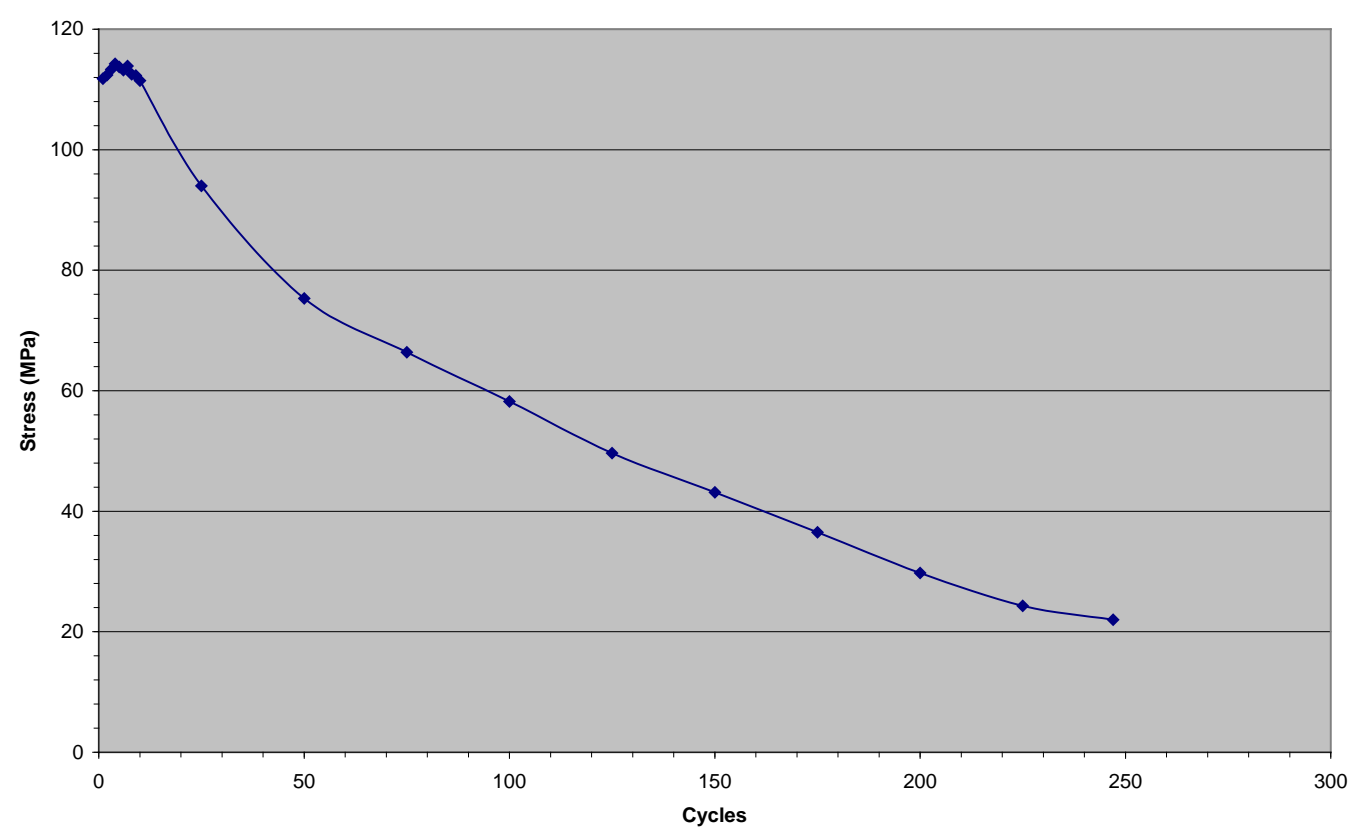

A22-isot Loading Cycles

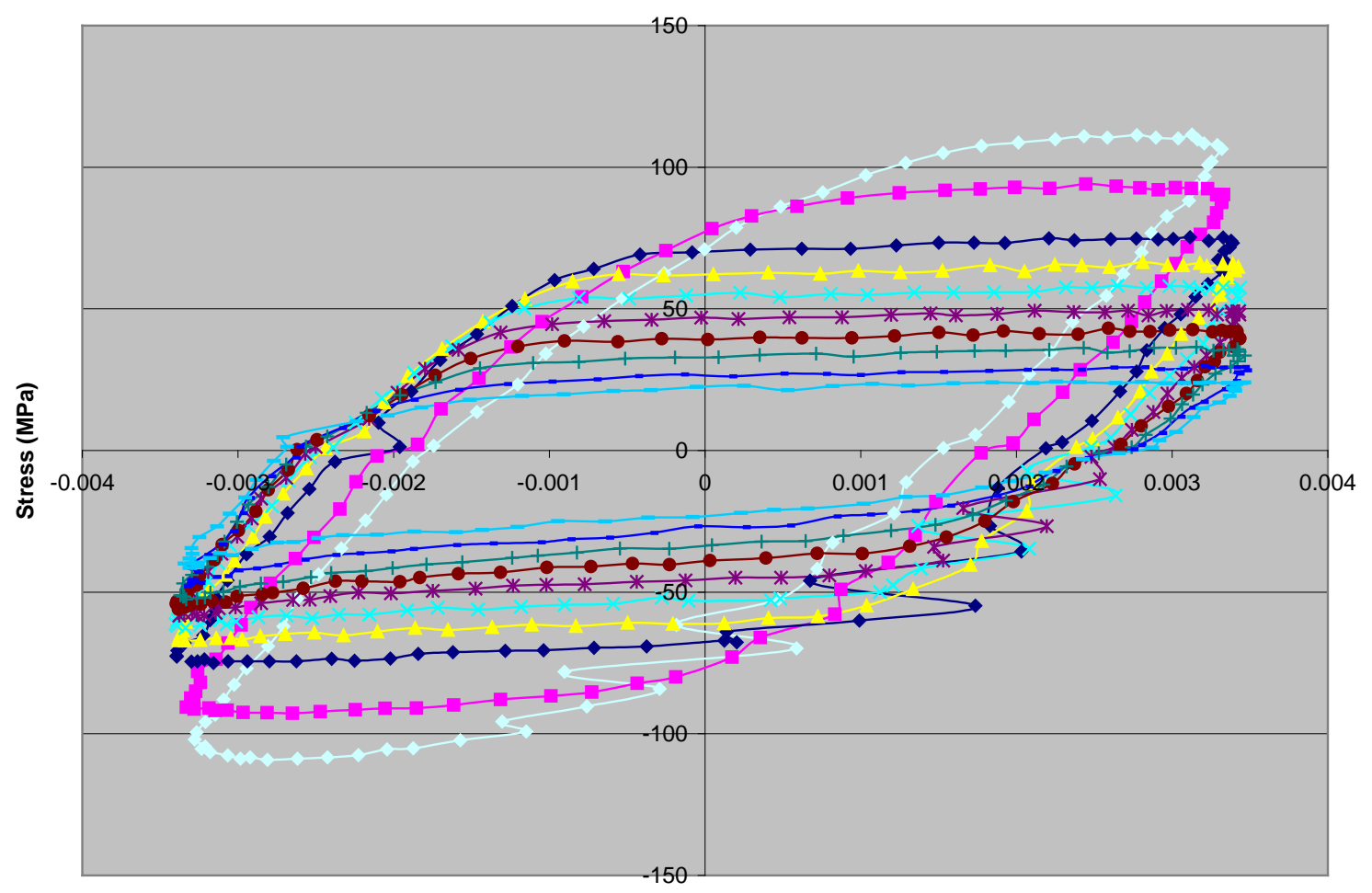

Strain $(\mathrm{mm} / \mathrm{mm})$ 


\section{A17}

These data pertain to $1350-\mathrm{H} 19$ at room temperature subjected to cyclic loading. 


\section{Appendix B}

\section{C24}

These data pertain to 6061-T6 at 200 degrees C subject to cyclic loading.

\section{c24-isot Loading Cycles}

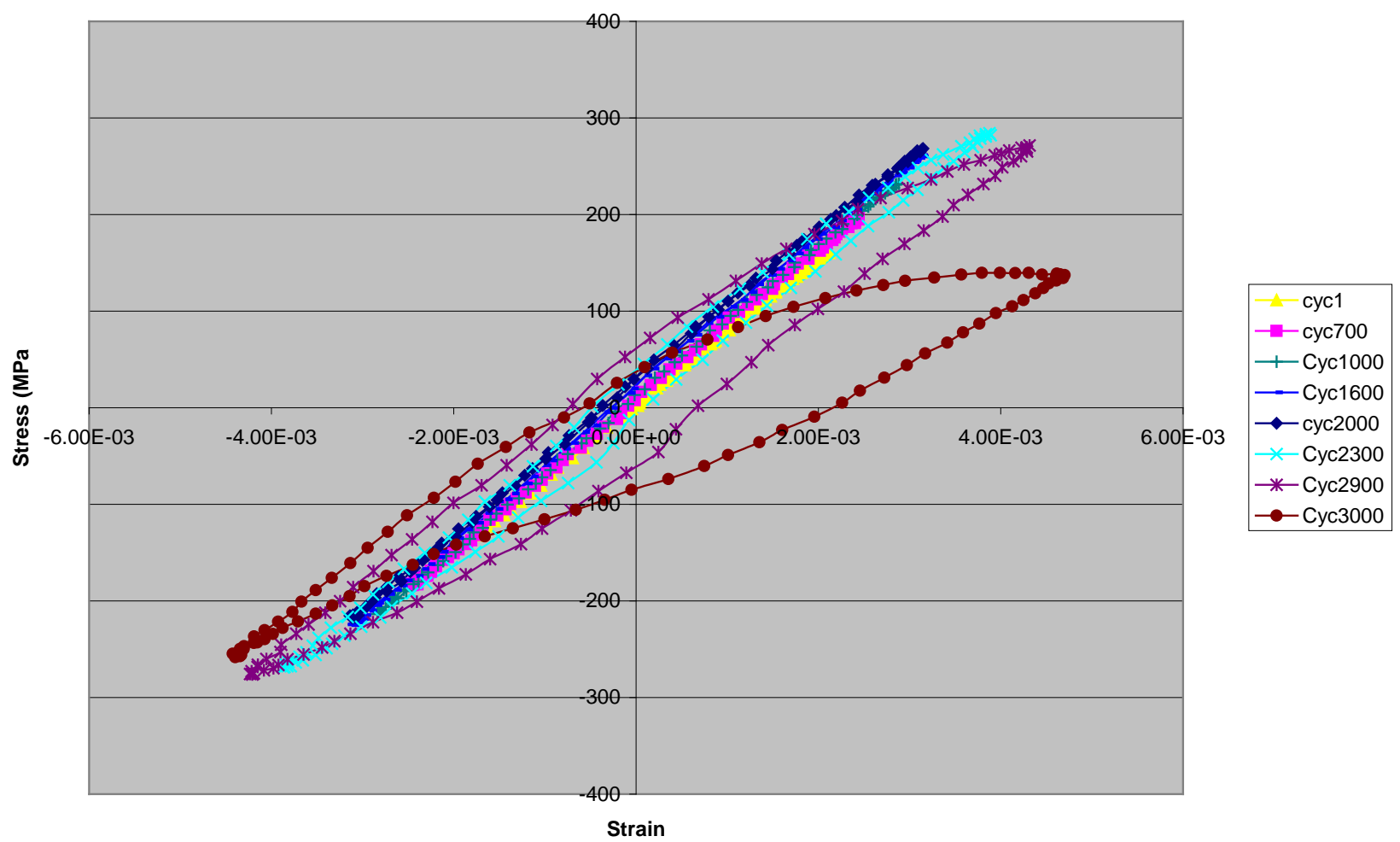

C24 Max Cycle Stress

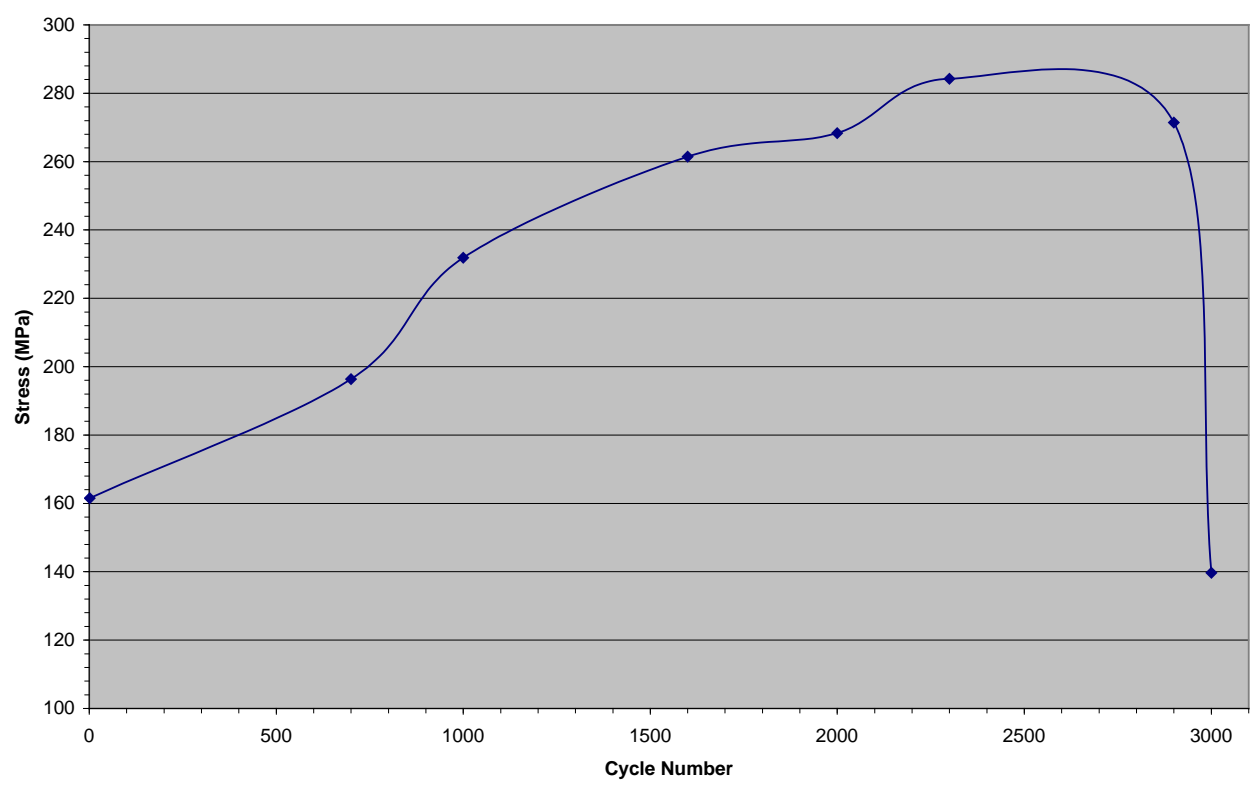


C24 Max Cycle Strain

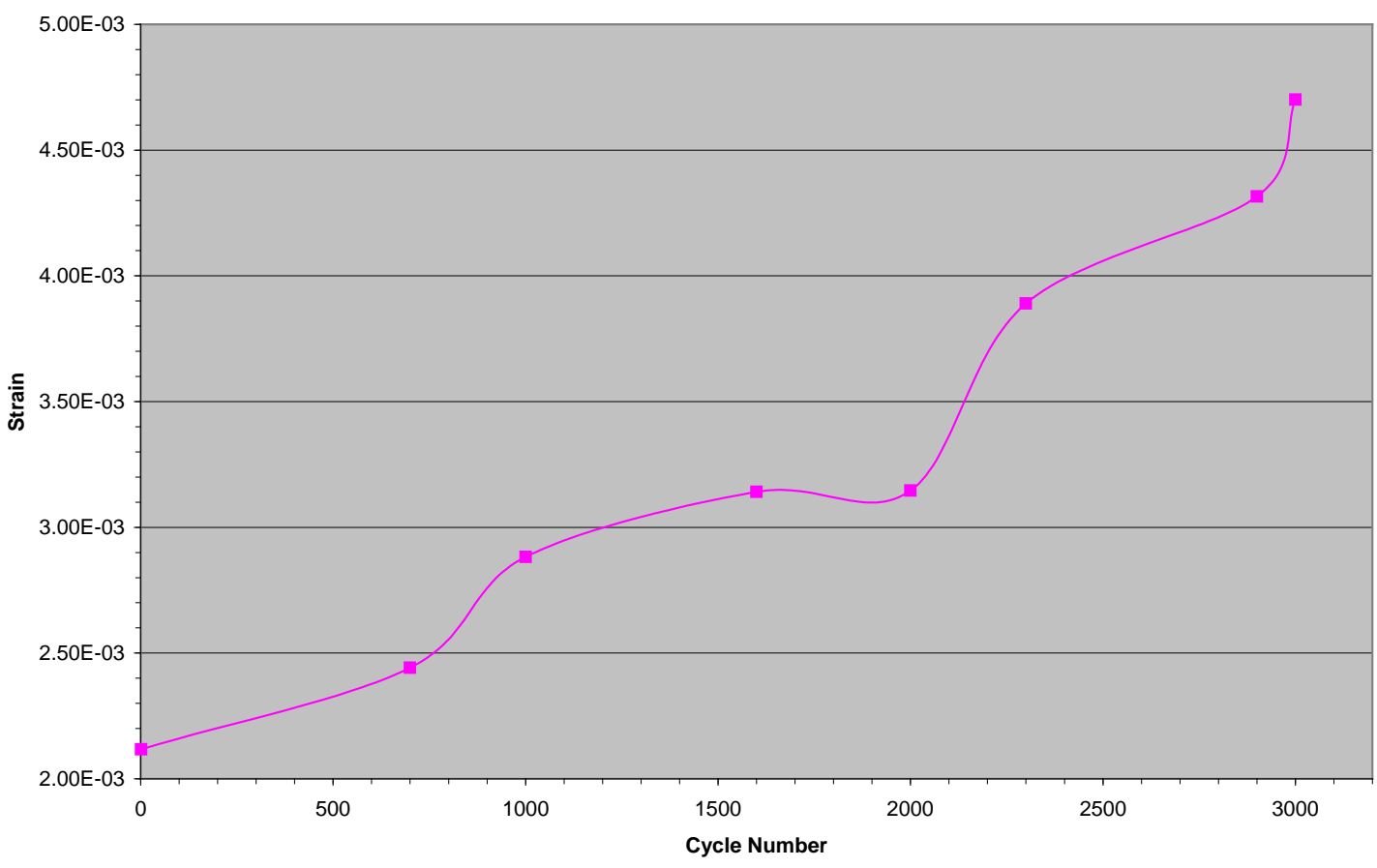

C24 Stiffness

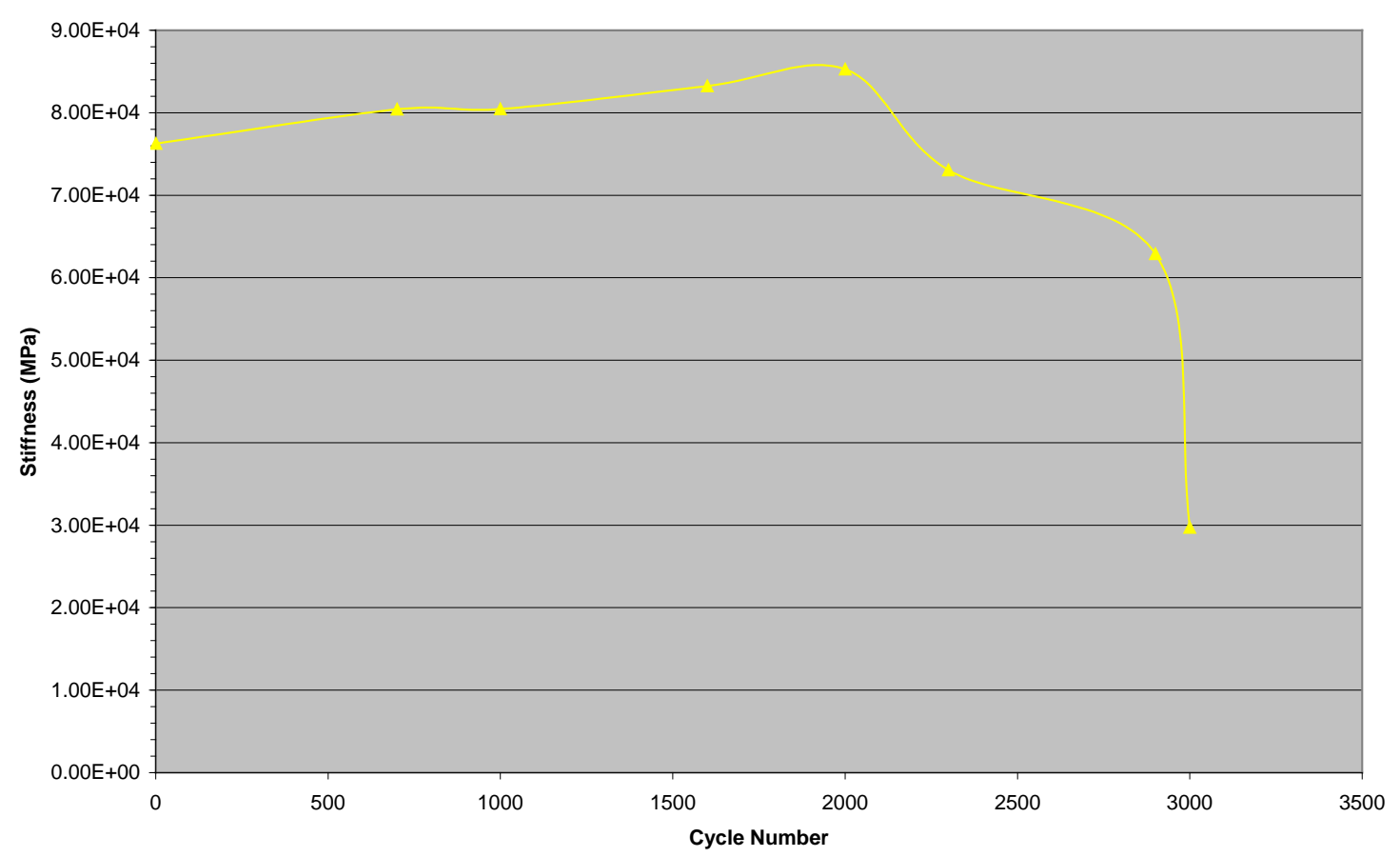




\section{Appendix B}

\section{9. $\underline{\text { N1-05-RT }}$}

These data pertain to $1350-\mathrm{H} 19$ at room temperature subject to cyclic loading.

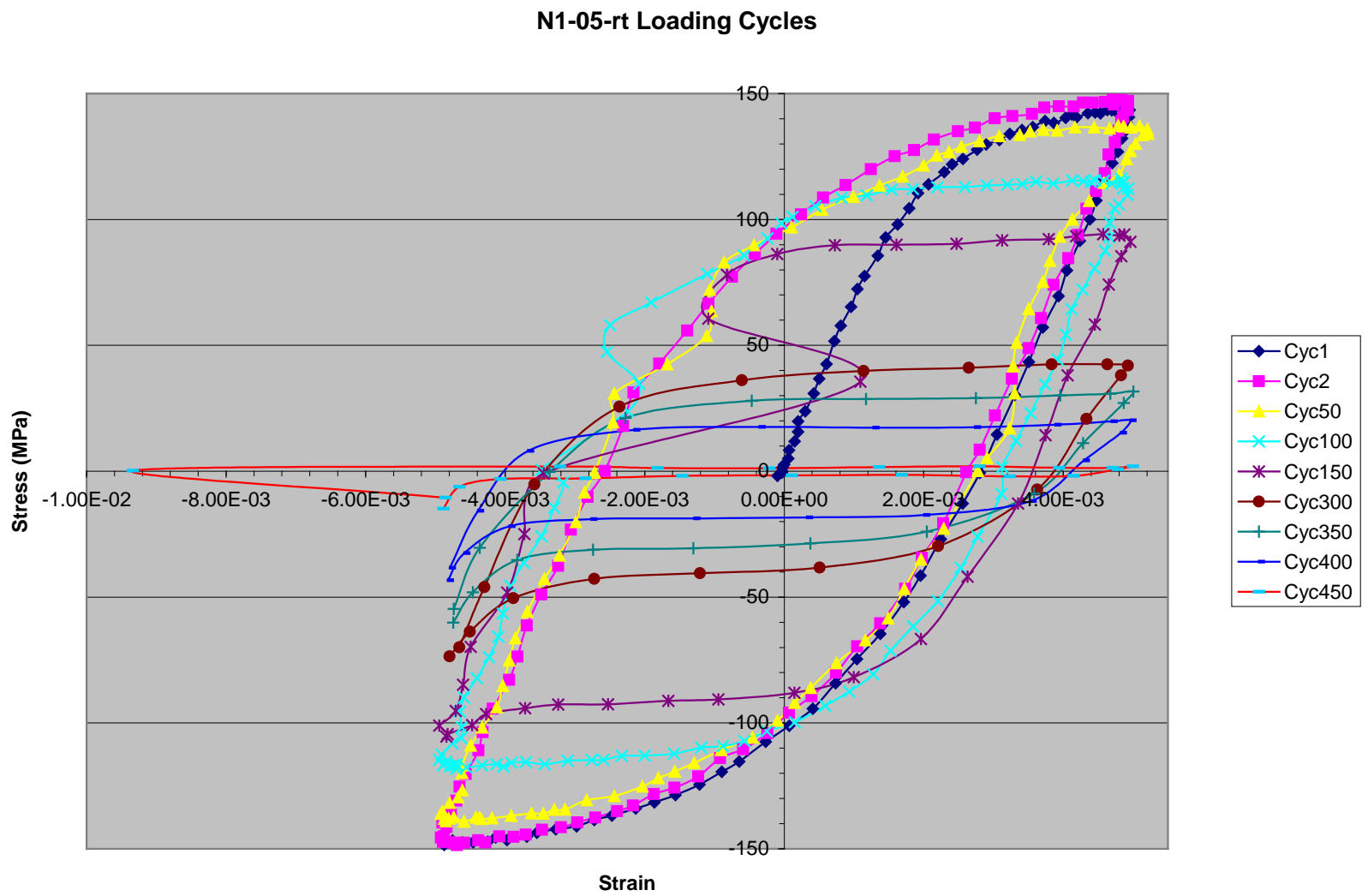

N1-05-rt Max Cycle Stress

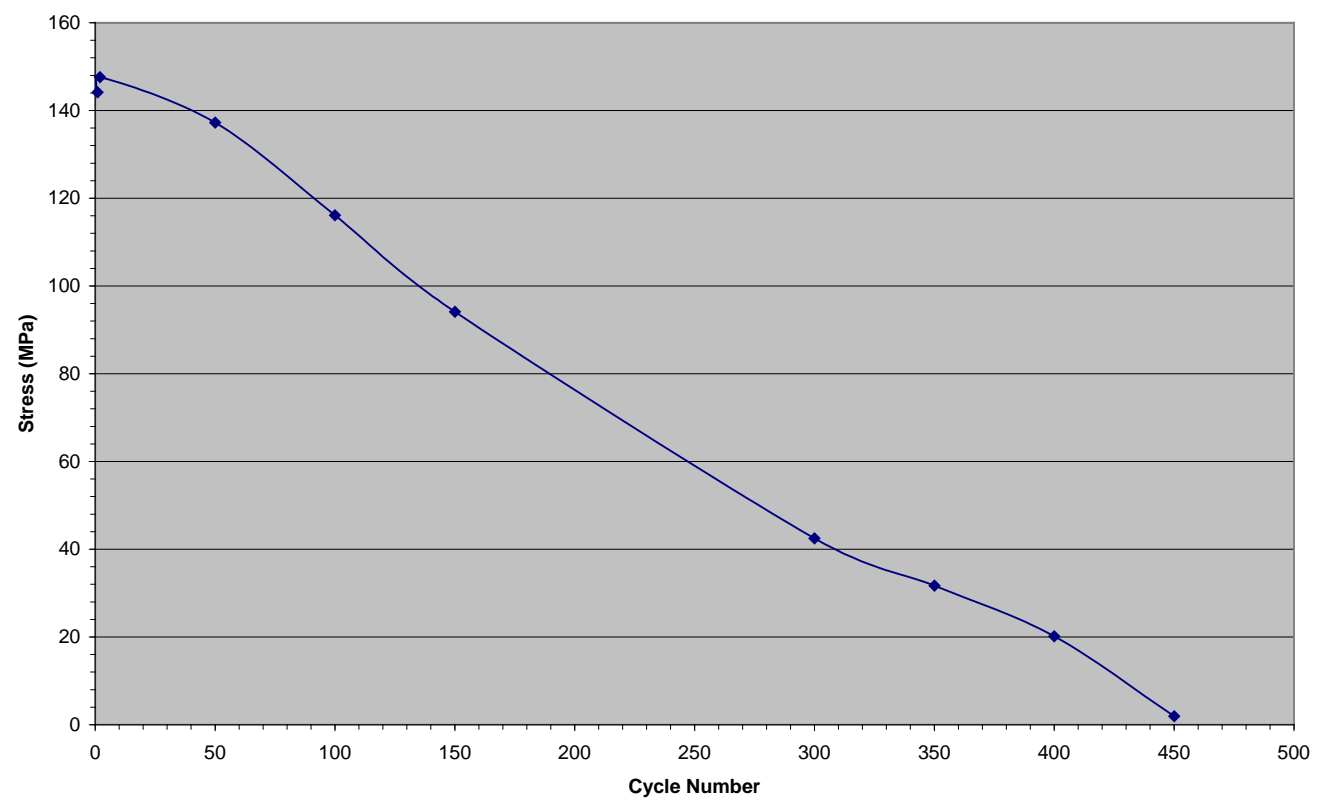


N1-05-rt Max Cycle Strain

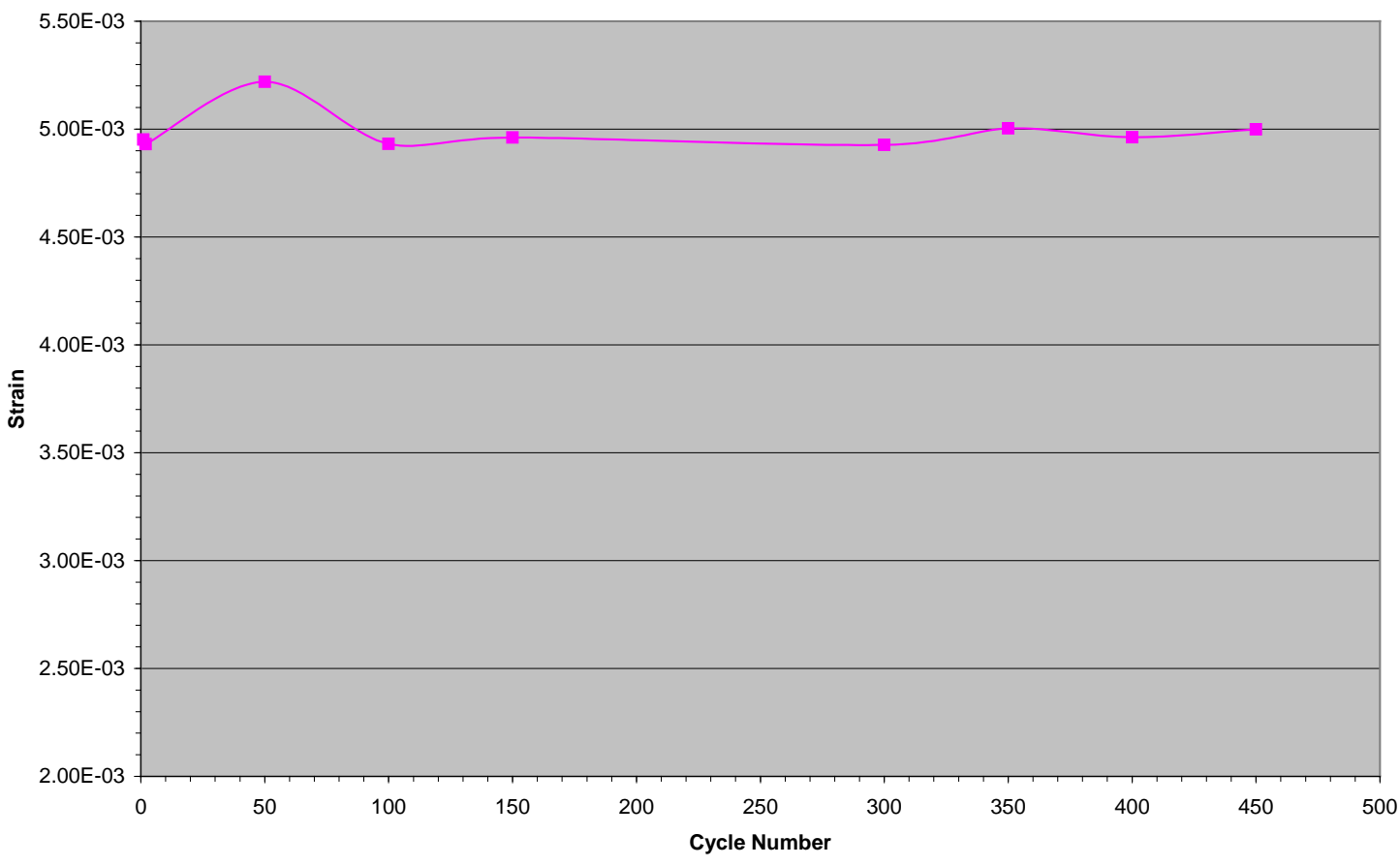




\section{N2-03-RT}

These data pertain to $1350-\mathrm{H} 19$ at room temperature subject to cyclic loading.

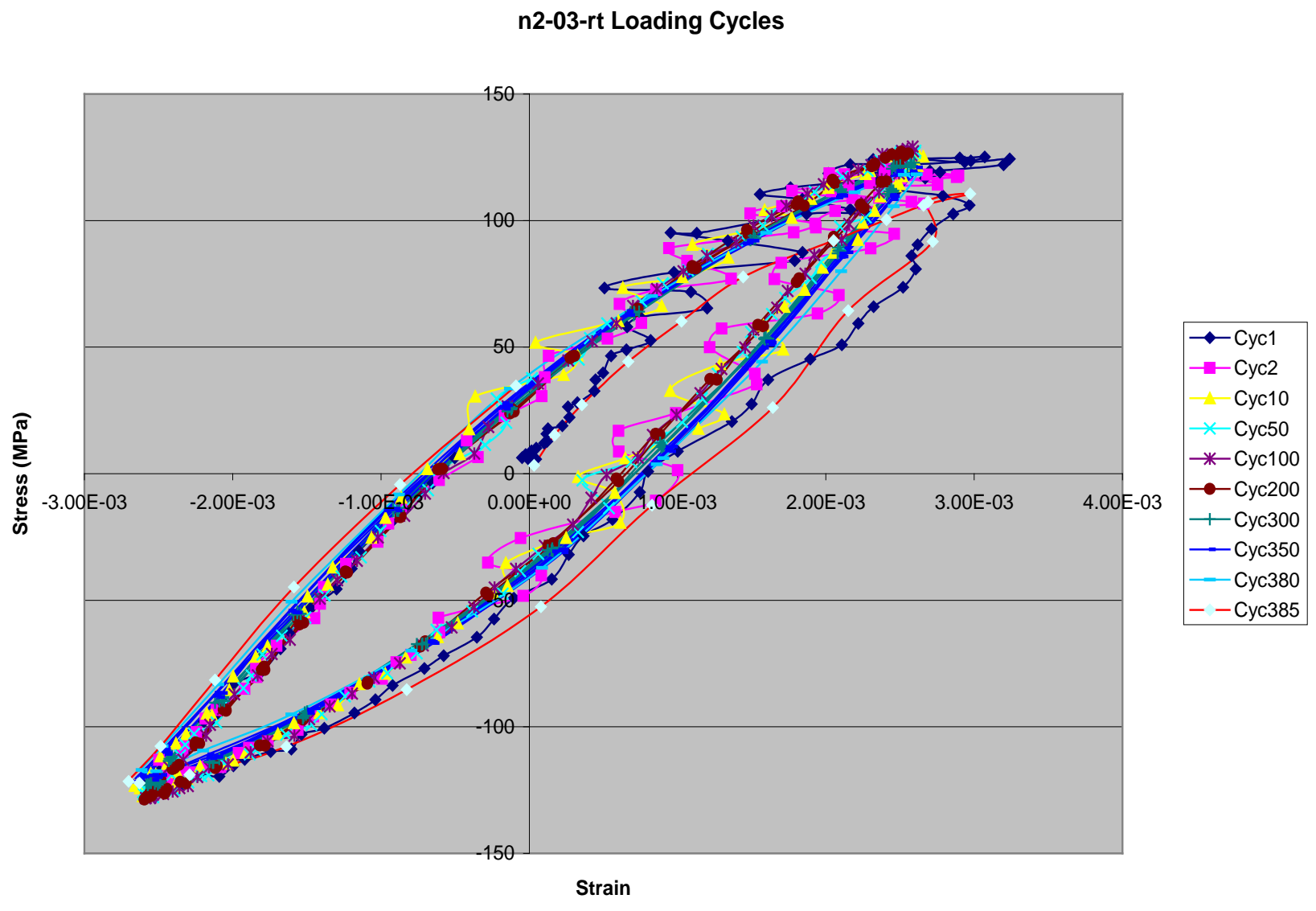


n2-03-rt Max Cycle Stress

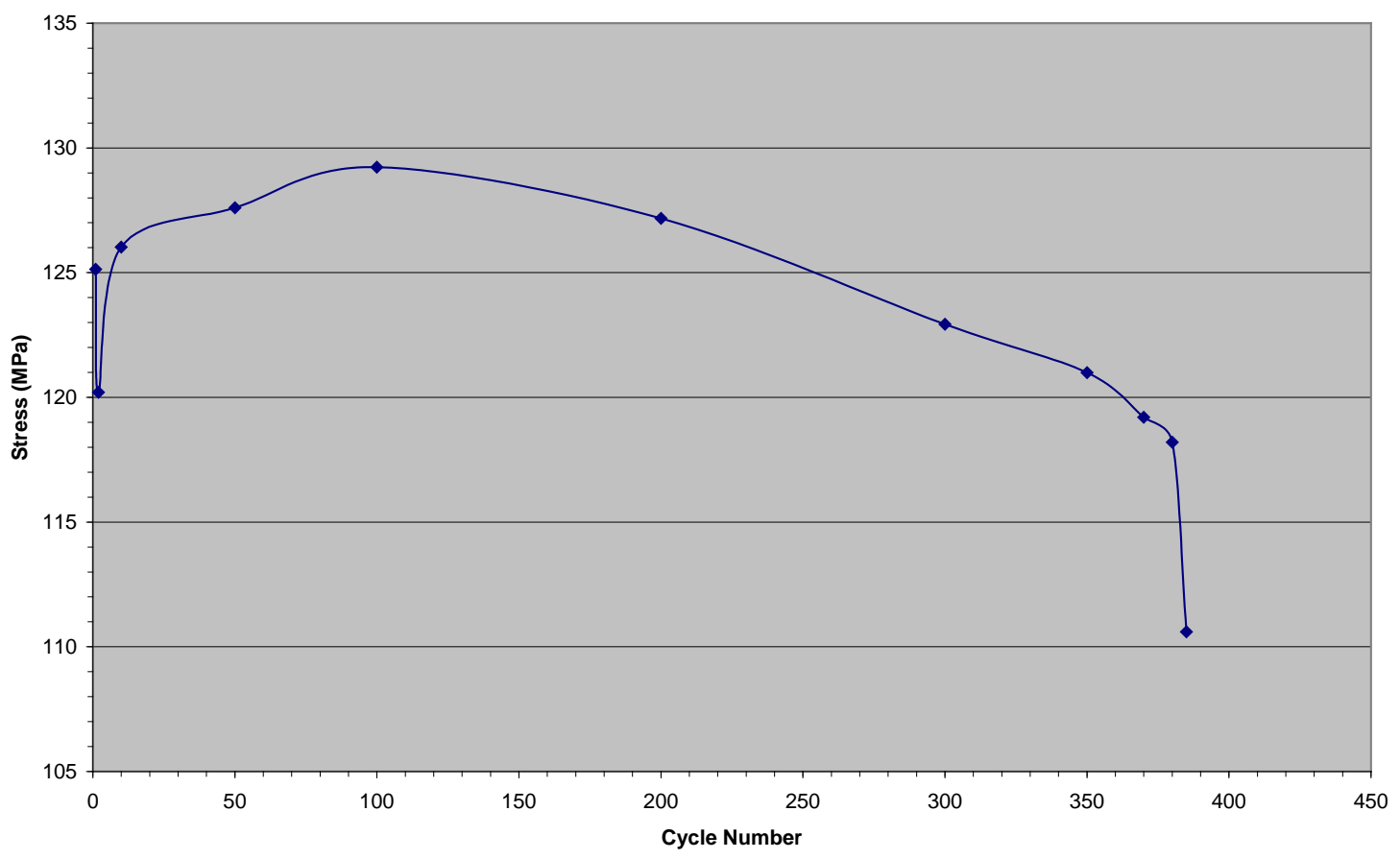

n2-03-rt Max Cycle Strain

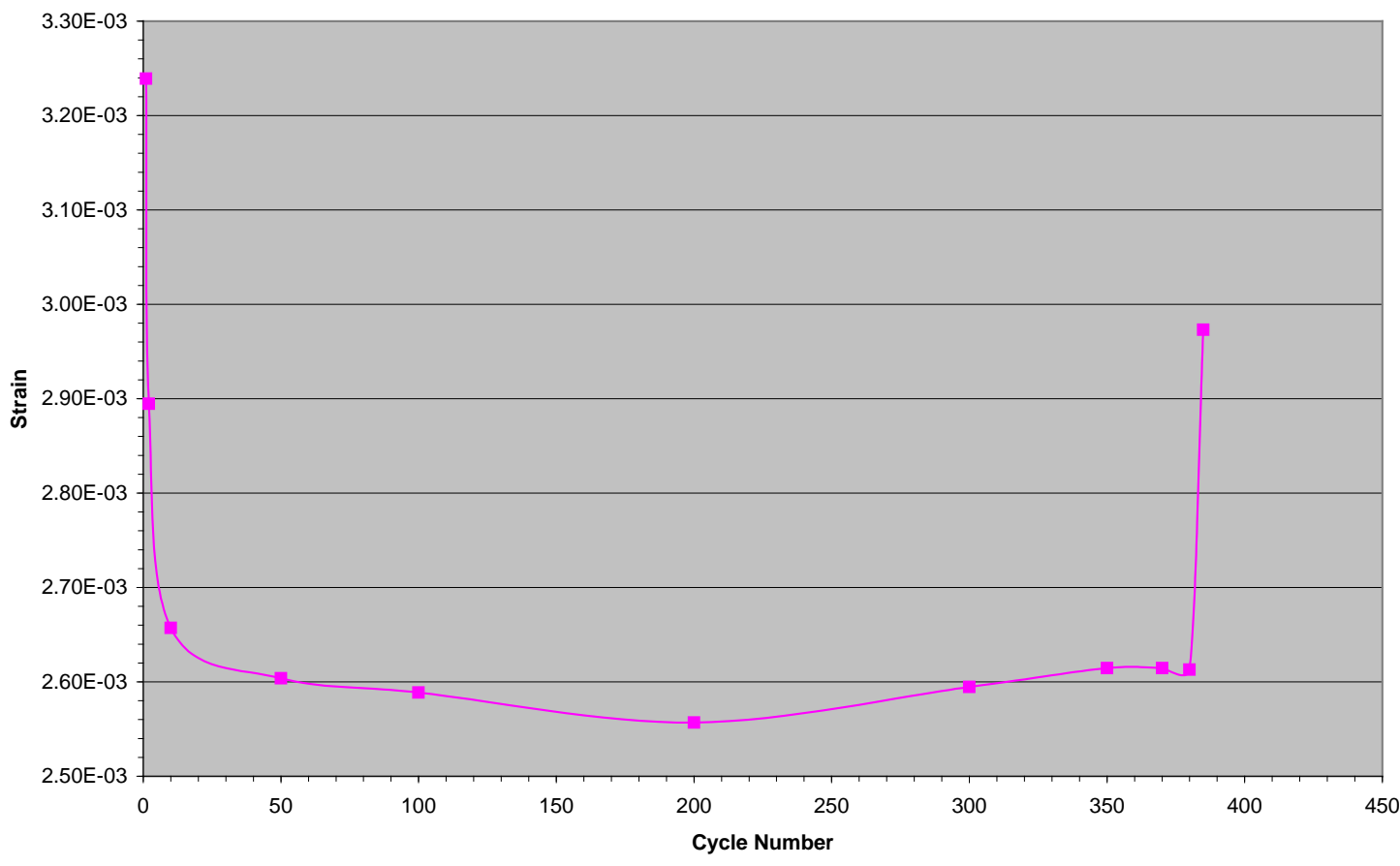

C-31 


\section{N3-001-rt}

These data pertain to 1350-H19 at room temperature subject to cyclic loading.

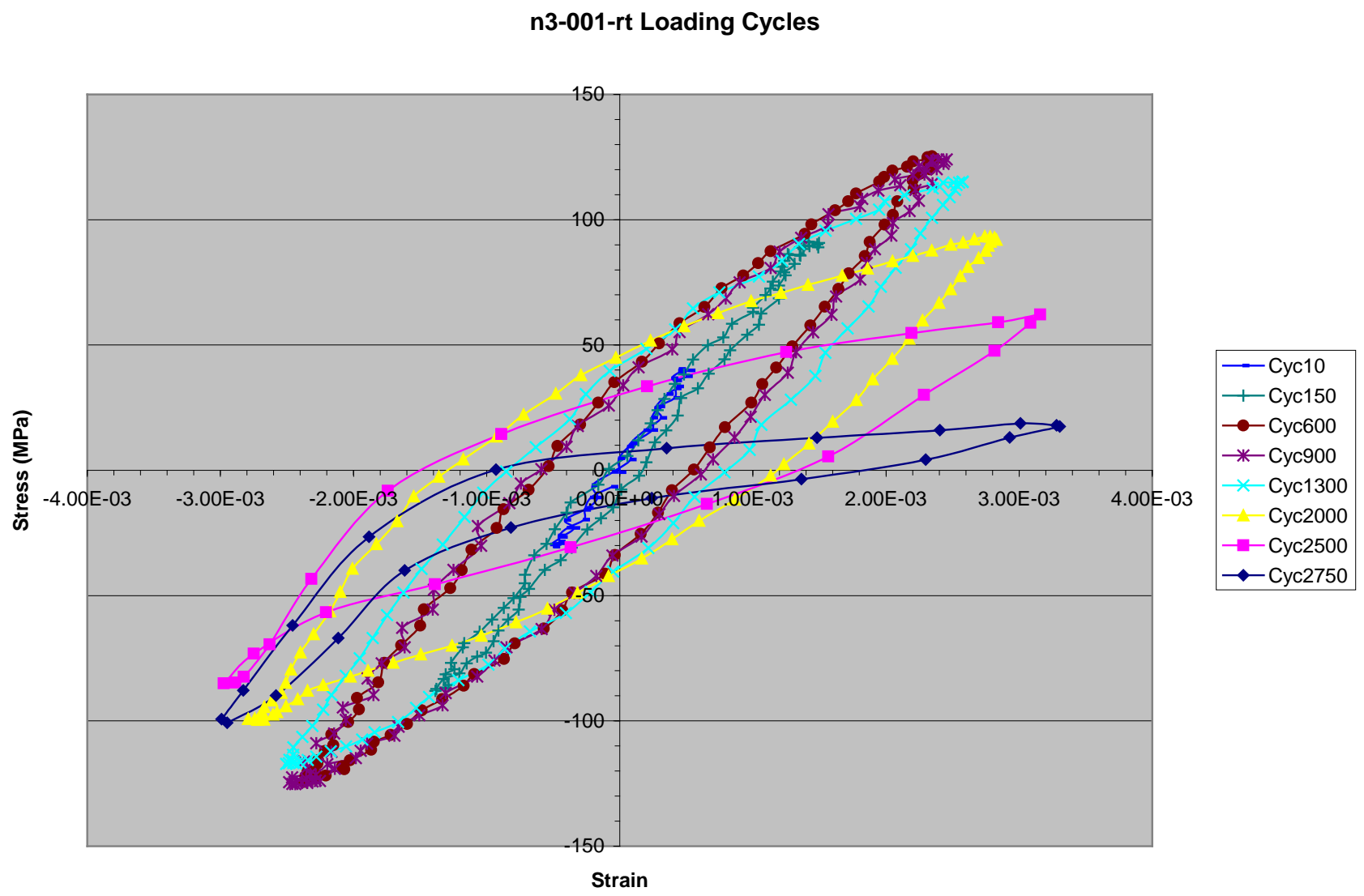


n3-001 Max Cycle Stress

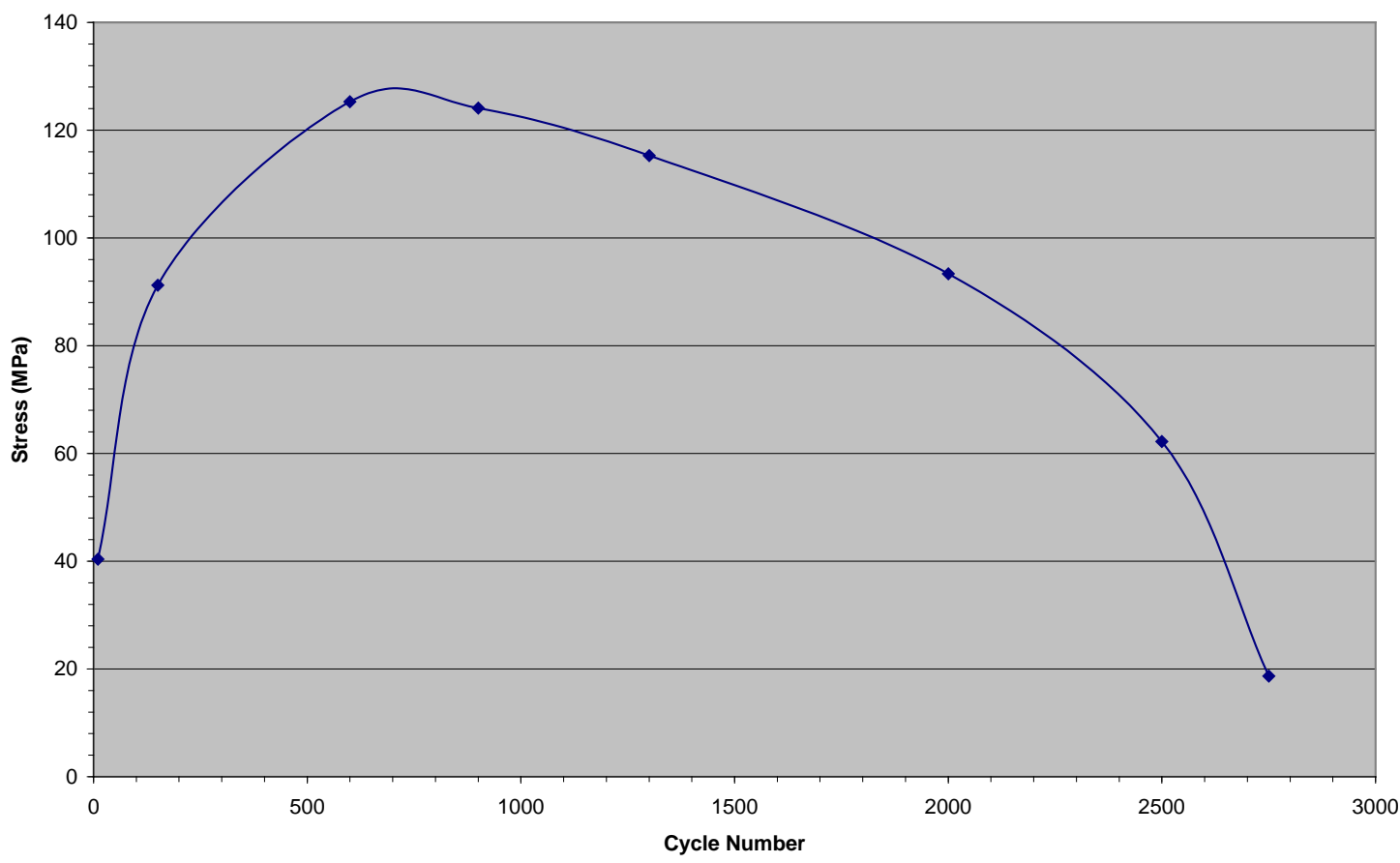

n3-001 Max Cycle Strain

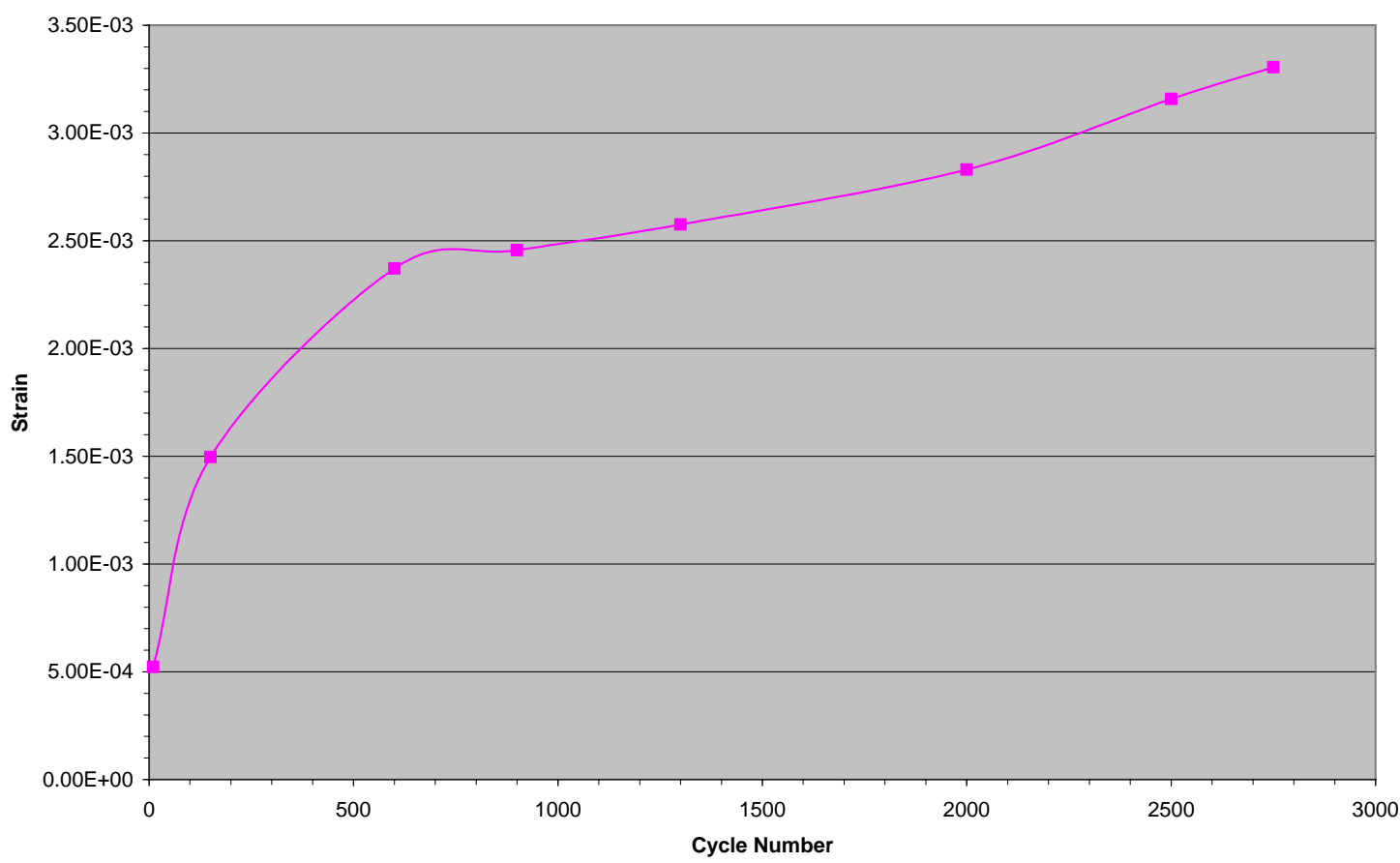

C-33 


\section{C.3 Thermal-Mechanical Fatigue (TMF) Test Results}

Experimental data from TMF cycle experiments was analyzed using Microsoft Excel and Mathematica 5.2 software. Each TMF data file contains information about the time, displacement, load, strain, temperature. The specimen diameter of 0.18 in was used in all experiments. The cross sectional area for each specimen was $16.417 \mathrm{~mm}^{2}$. The load in $\mathrm{N}$ when divided by this area gives stress in MPa at each data point.

\section{1. $\underline{\text { A16-tmf }}$}

These data pertain to $1350-\mathrm{H} 19$ subjected to TMF cyclic loading, $100^{\circ} \mathrm{C}-150^{\circ} \mathrm{C}$. The total strain was also cycle between 0 and $0.3 \%$.

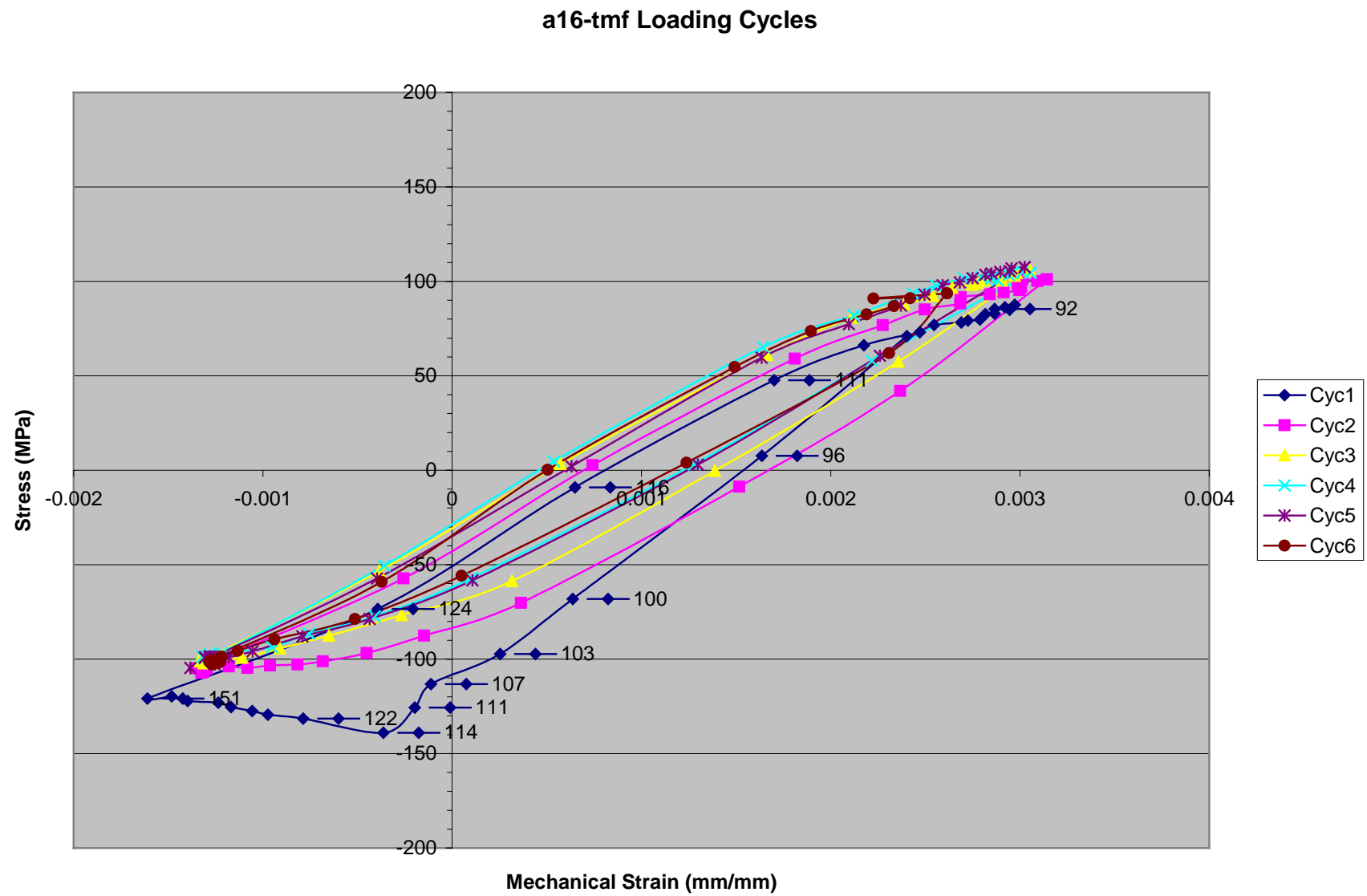




\section{A20}

These data pertain to 1350-H19 subjected to TMF cyclic loading. 100-150C

The total strain was cycle 0 to $0.2 \%$.

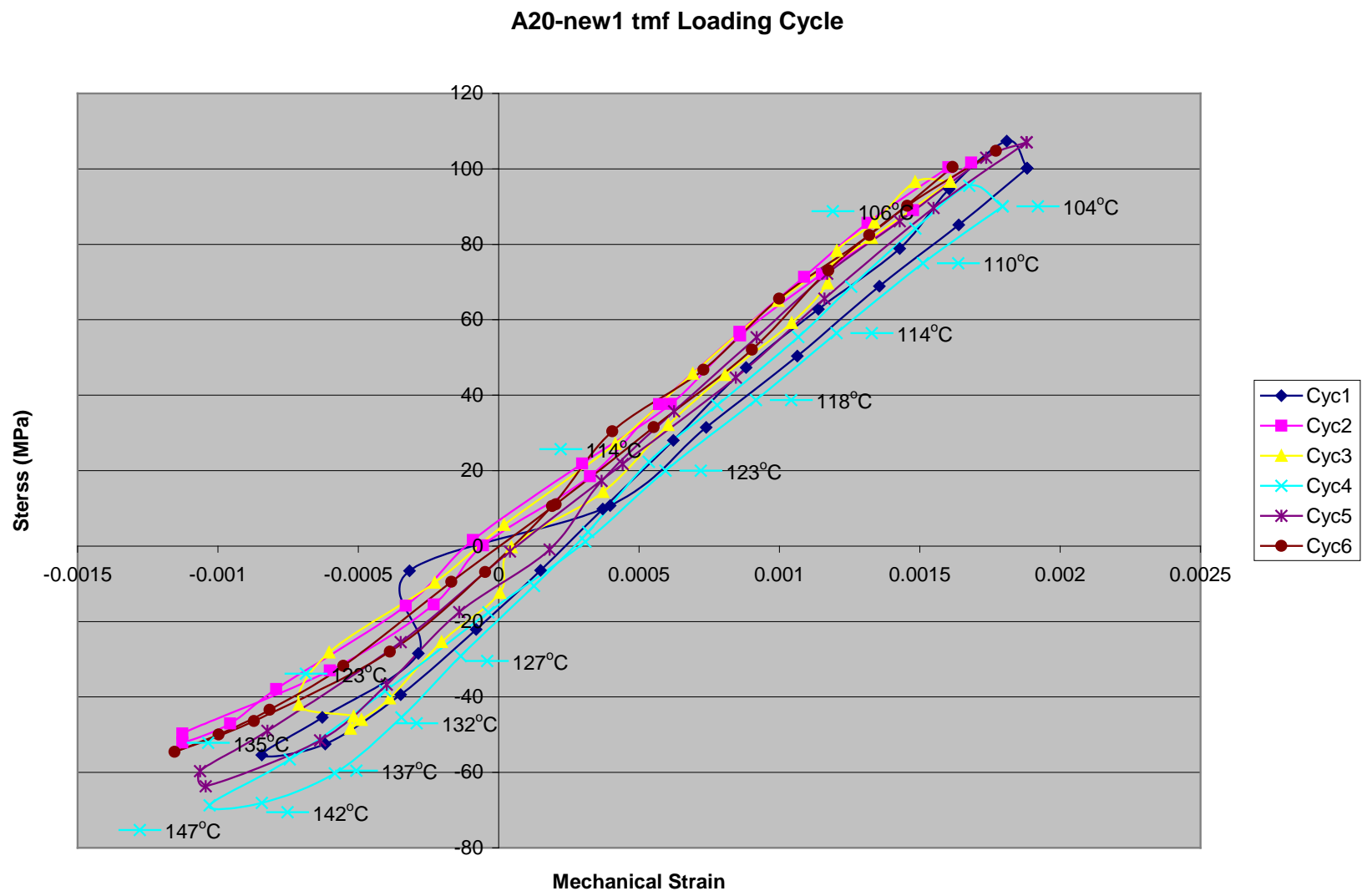


A20new2 TMF Loading Cycles

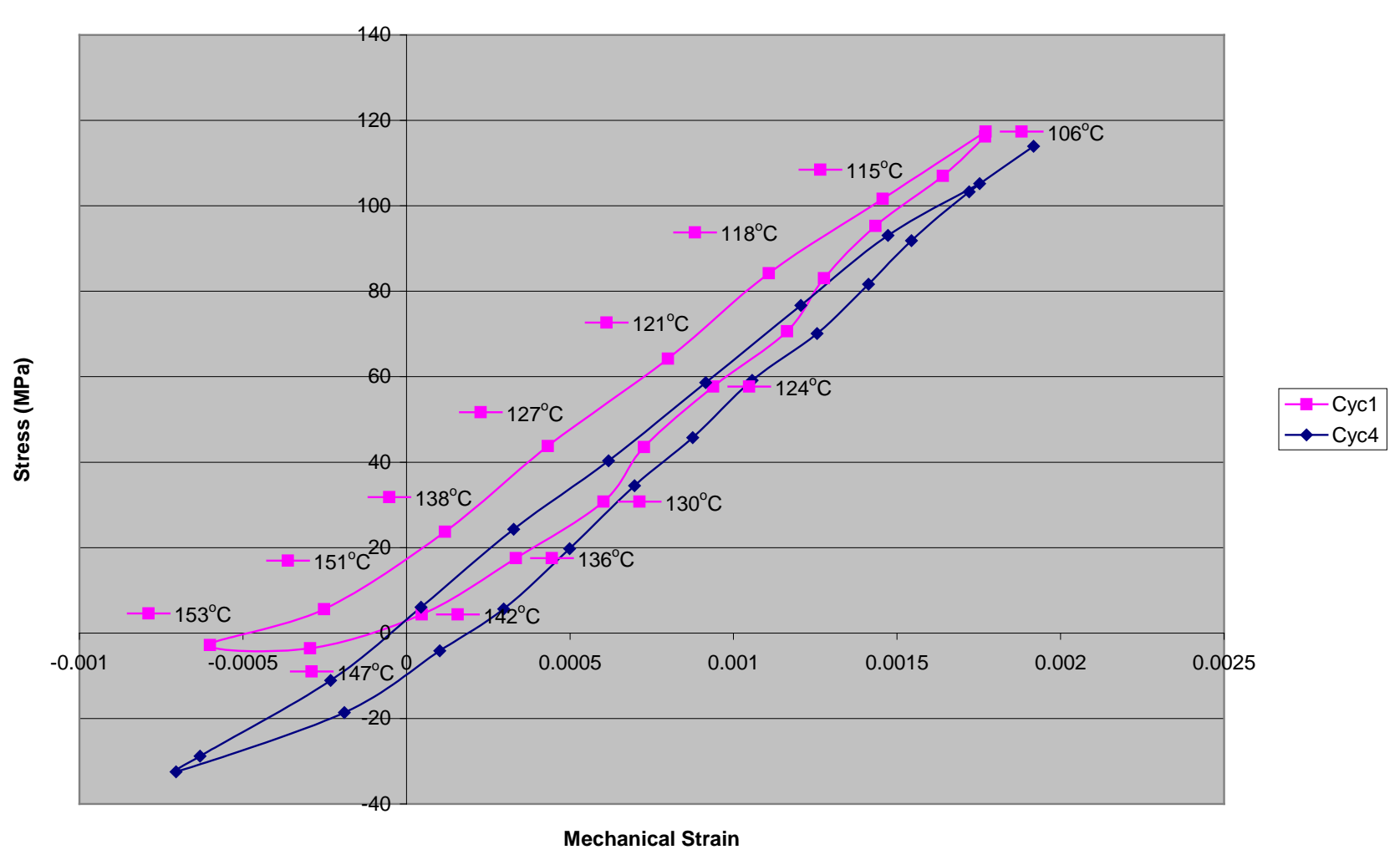




\section{A21}

These data pertain to $1350-\mathrm{H} 19$ material subjected to TMF cyclic loading. $\sim 85^{\circ} \mathrm{C}-155^{\circ} \mathrm{C}$.

The total strain was maintained at close to $0.3 \%$

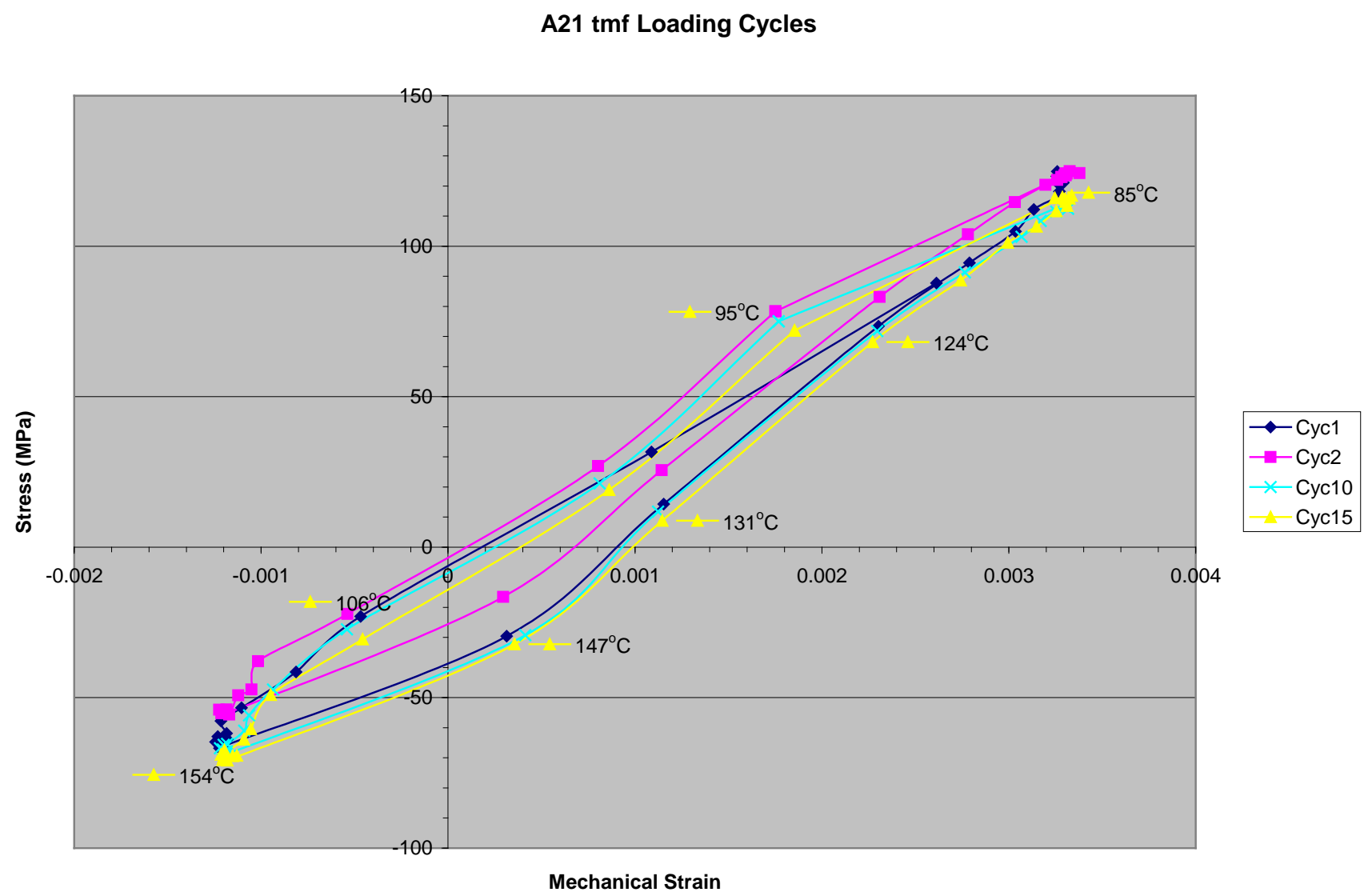




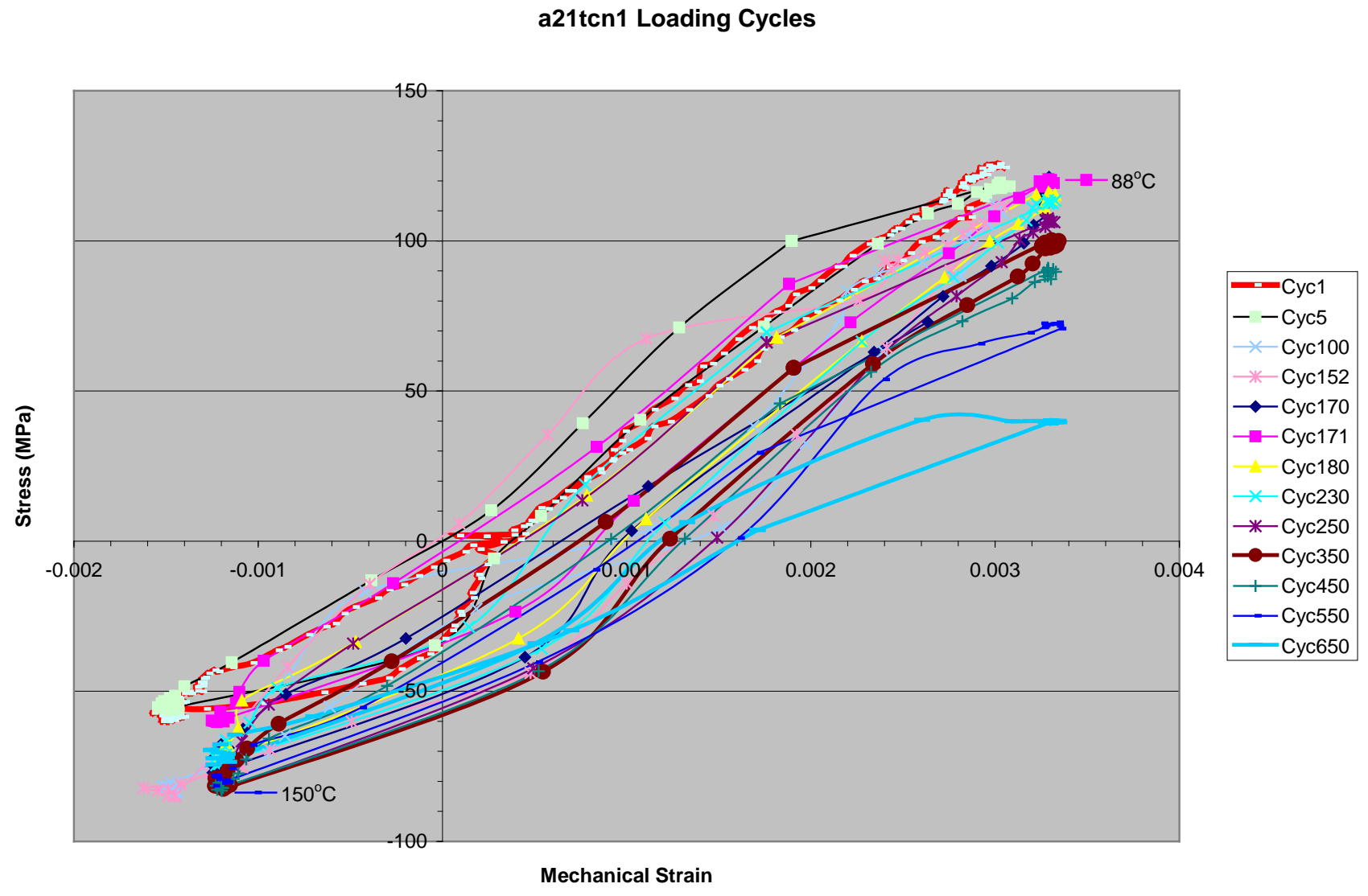




\section{4. $\mathrm{N9}$}

These data pertain to $1350-\mathrm{H} 19$ subjected to TMF cyclic loading. The total strain was maintained at close to zero. The thermal strain was subtracted from the total strain to obtain the mechanical strain.

\section{n9-tmf Loading Cycles}

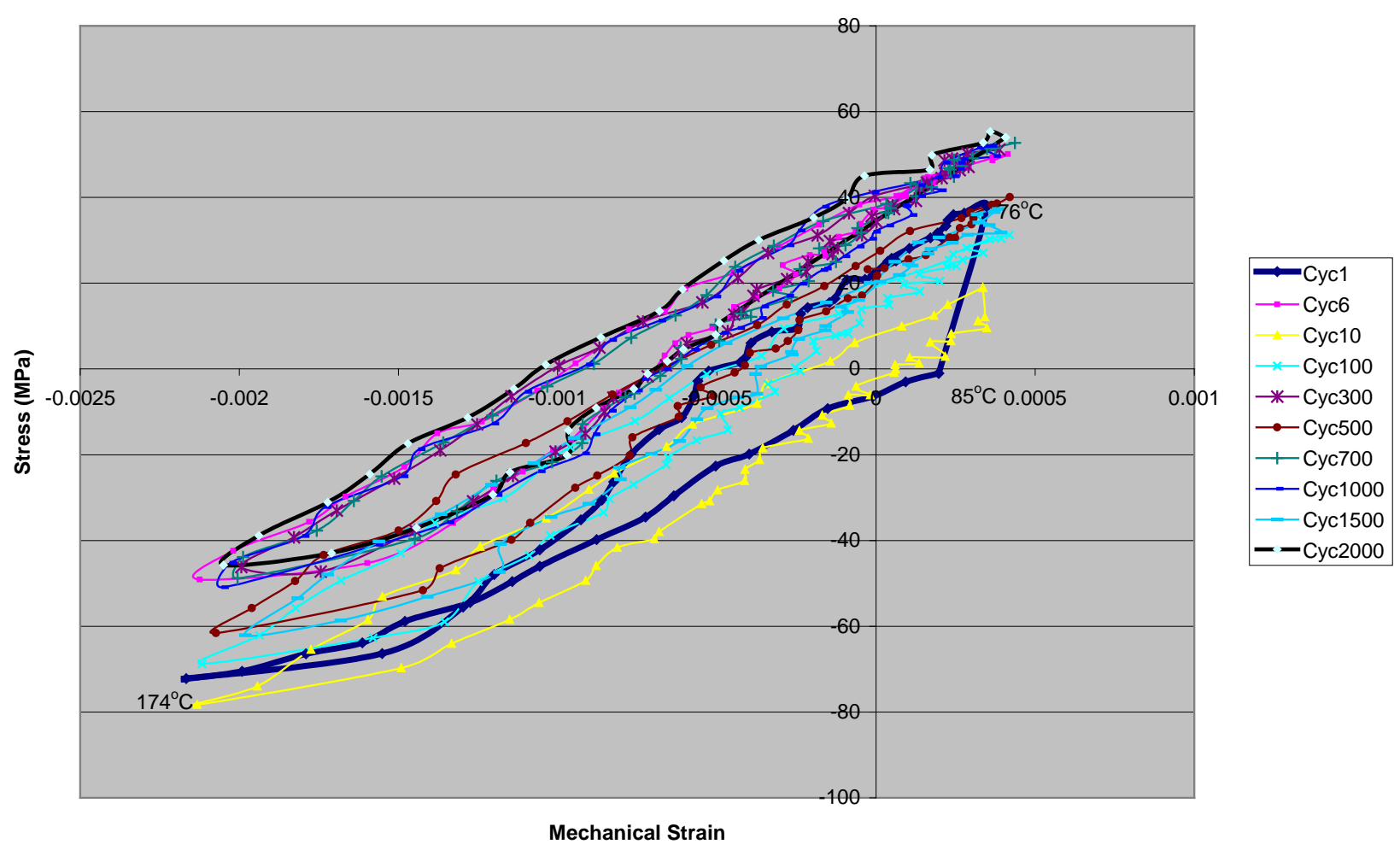




\section{5. $\underline{\text { C9-tmf }}$}

These data pertain to $6061-\mathrm{T} 6$ material subjected to TMF cyclic loading. $75^{\circ} \mathrm{C}$ to $180^{\circ} \mathrm{C}$.

The total strain was maintained at close to zero although the test.

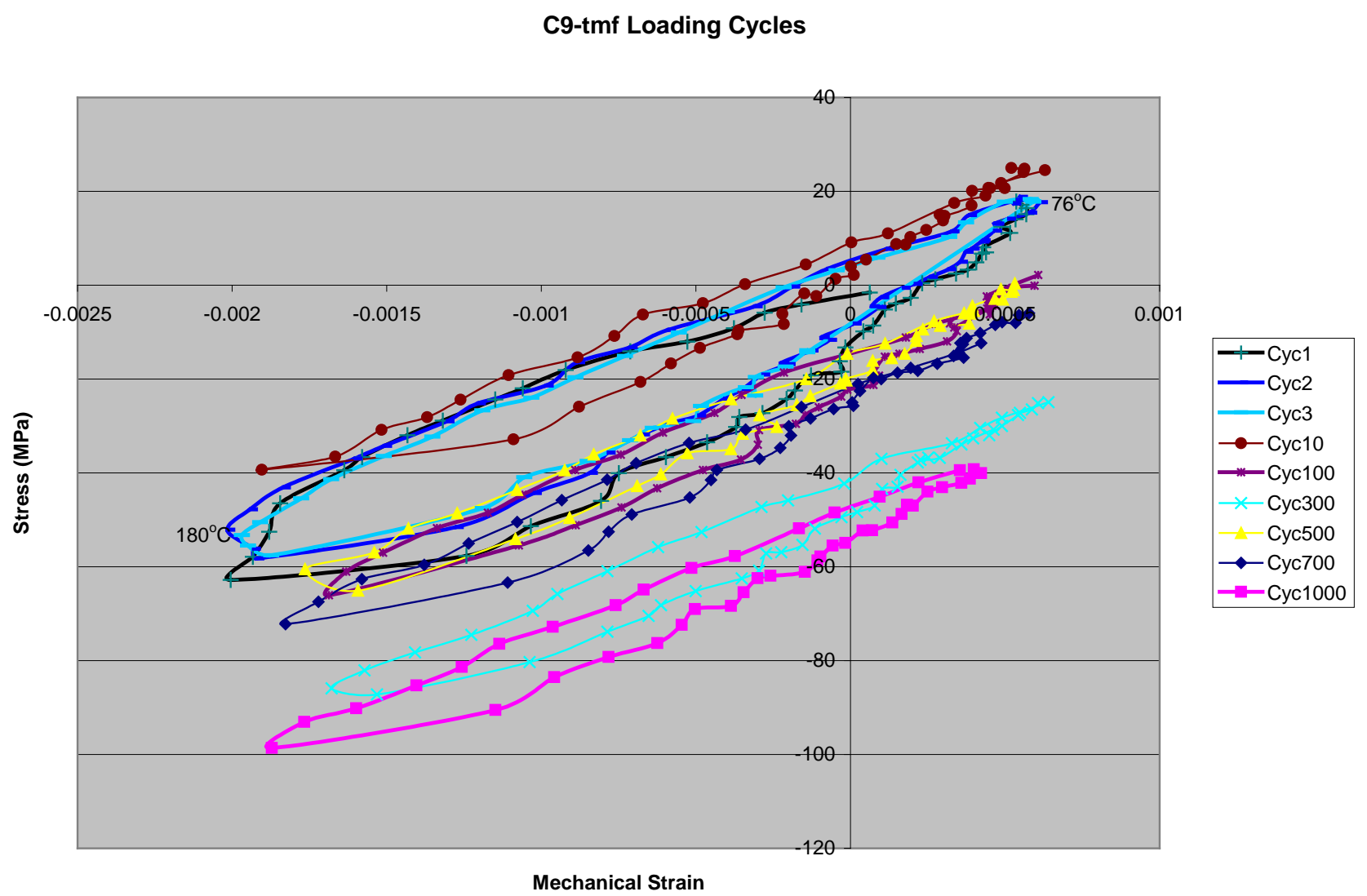




\section{N19}

These data pertain 1350-H19 subjected to TMF loading cycles. The total strain was maintained at $0.2 \%$ while temperature was cycled from $100^{\circ} \mathrm{C}$ to $150^{\circ} \mathrm{C}$.

\section{N19-tmf Loading Cycles}

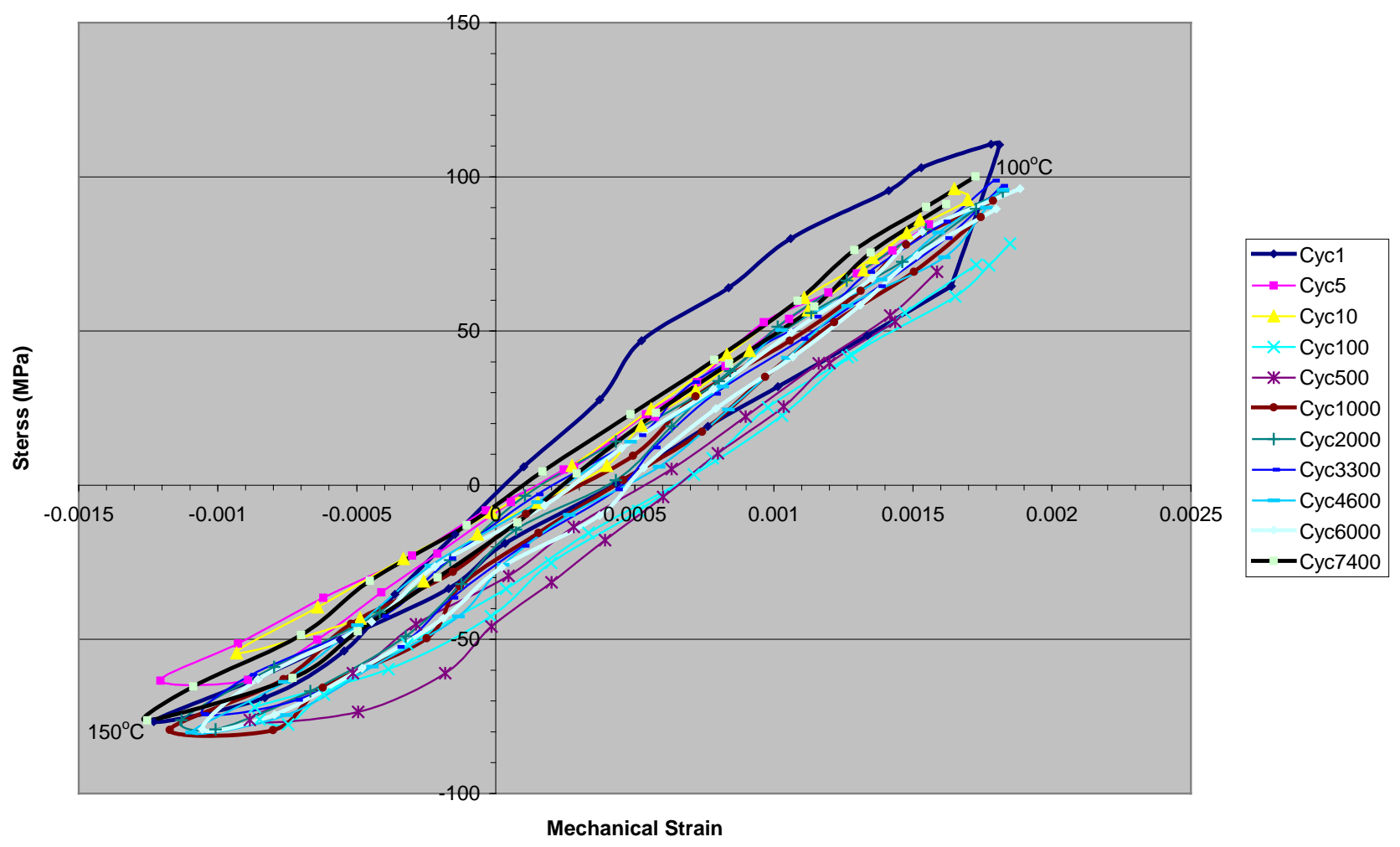




\section{N24}

These data pertain to 1350-H19 material subjected to TMF cyclic loading. The total strain was maintained very close to zero the temperature of the material cycled between $100^{\circ} \mathrm{C}$ and $150^{\circ} \mathrm{C}$.

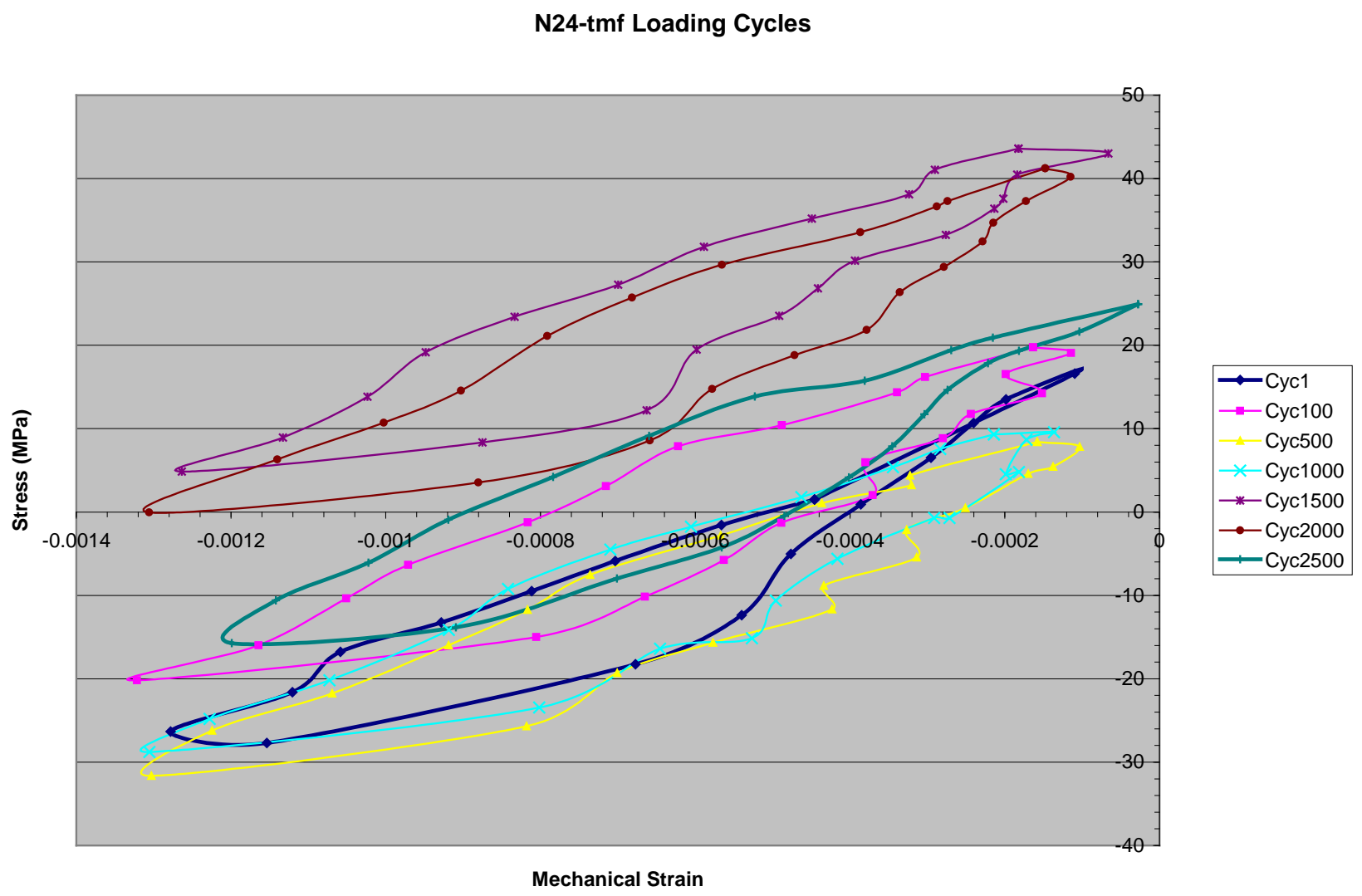




\section{A12}

These data pertain to 1350-H19 subjected to TMF cyclic loading. The temperature of material was cycled from $100^{\circ} \mathrm{C}$ to $150^{\circ} \mathrm{C}$ out of phase with the variation of the total strain from 0 to $0.2 \%$.

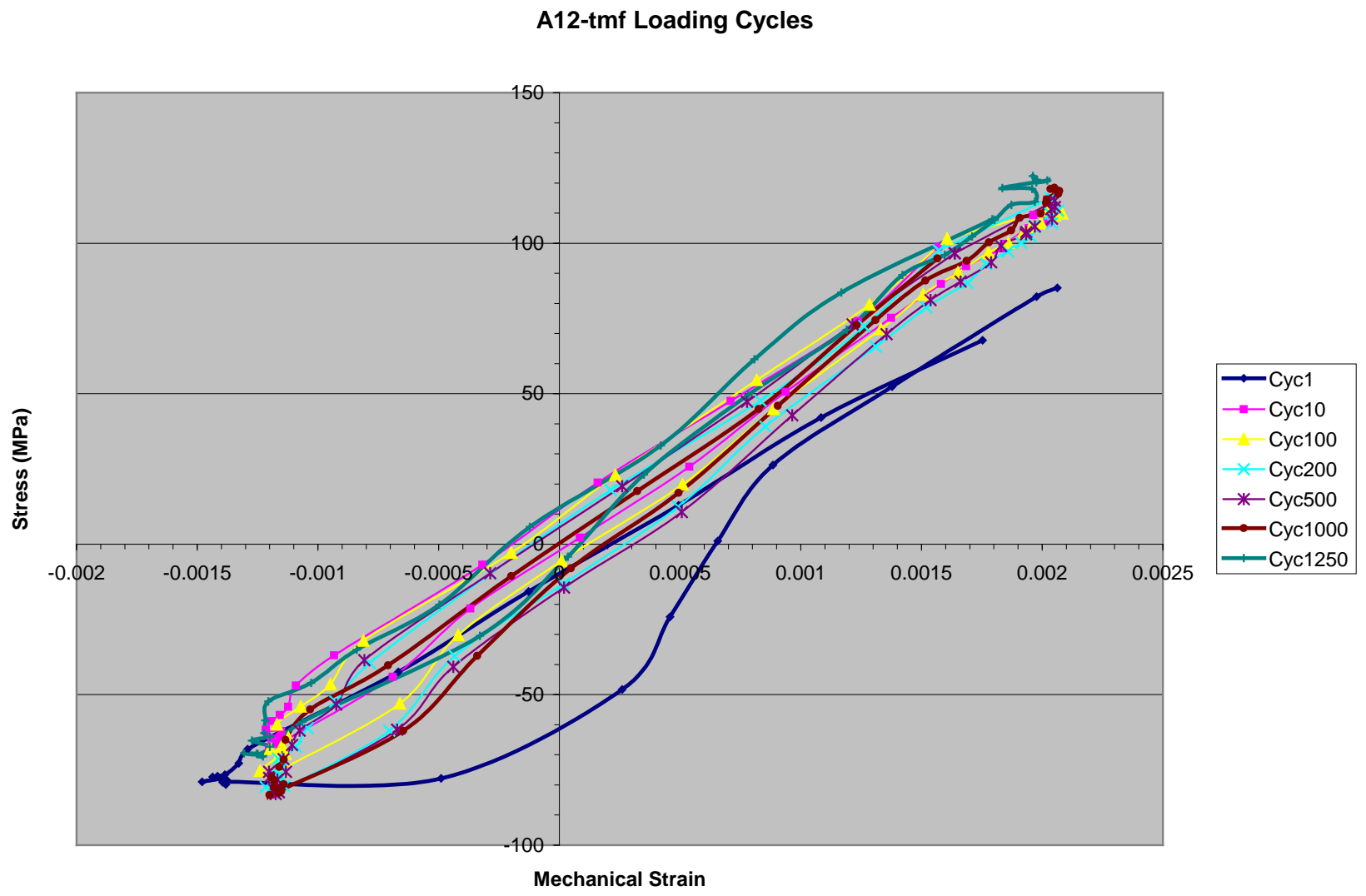




\section{A13-tmf}

These data pertain to 1350-H19 material subjected to TMF cyclic loading. The temperature was cycled from $100^{\circ} \mathrm{C}$ to $150^{\circ} \mathrm{C}$ out of phase with strain, while the total strain was varied between 0 and $0.2 \%$.

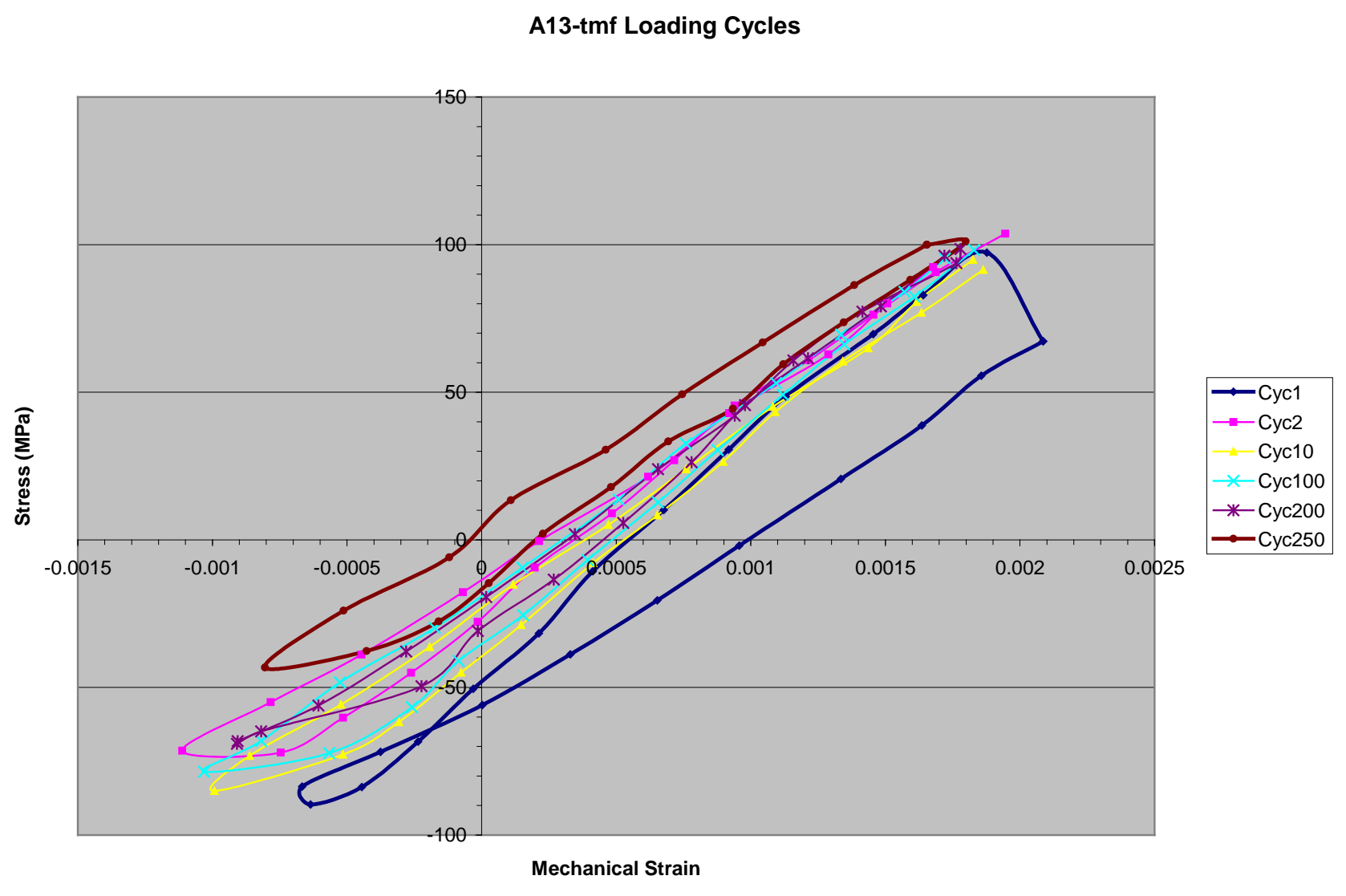




\section{$D$ CONIDERATIONS OF THERMAL CYCLING EXPERIMENTS FOR SINGLE STAGE SPLICE CONNECTOR FITTING}

\section{D.1 Issues Associated with SSC Thermal Cycling Experiments and the Conductor Aging}

The subjects are described in the following three tables.

Table 1: Thermal couples location in SSC system

\begin{tabular}{|l|l|}
\hline Title & $\begin{array}{l}\text { Issues Associated with Temperature Measurement and the Single-Stage Splice } \\
\text { Connector Aging }\end{array}$ \\
\hline Description & $\begin{array}{l}\text { High temperature and high current operations create added mechanical, electrical and } \\
\text { thermal stresses on the fitting. Compression fittings are the most susceptible } \\
\text { component of the conductor's thermal cycling; even though at the beginning of the } \\
\text { service life, the fittings are generally cooler than the conductor. Furthermore, with } \\
\text { annealed aluminum, it is difficult to obtain an adequate electrical connection between } \\
\text { strands and the barrel -due to aluminum oxides. The consequence of lack of good } \\
\text { contact is an increase in the connector temperature. Annealed strands also have an } \\
\text { increased creep rate which again allows them to flow away from the connection } \\
\text { pressure rather than have a residual stress against the connection. This is accentuated } \\
\text { with expansion and contraction cycles when the thermal dynamics of the loading } \\
\text { conductor varies. }\end{array}$ \\
$\begin{array}{l}\text { One additional source of the fitting temperature increase is due to the increase of } \\
\text { resistance by a "loosening" action of the connection that occurs with expansion and } \\
\text { contraction of the conductor and fittings during the thermal cycling. Therefore, with } \\
\text { sufficient crimping forces in the compression fittings, it provides good contact surface } \\
\text { between the conductor strands and the connector's current carrying parts. This can } \\
\text { significantly mitigate the connector aging. }\end{array}$ \\
$\begin{array}{l}\text { In order to have a practical evaluation of the splice connector's aging, a detailed } \\
\text { temperature profiles of the conductor-connector system are needed. The detailed } \\
\text { locations of the thermal couples are specified with black arrows shown in Fig. 1, } \\
\text { where: a-conductor surface, b-steel core, c-steel core and aluminum conductor } \\
\text { interface, d-splice connector surface. }\end{array}$ \\
\hline
\end{tabular}




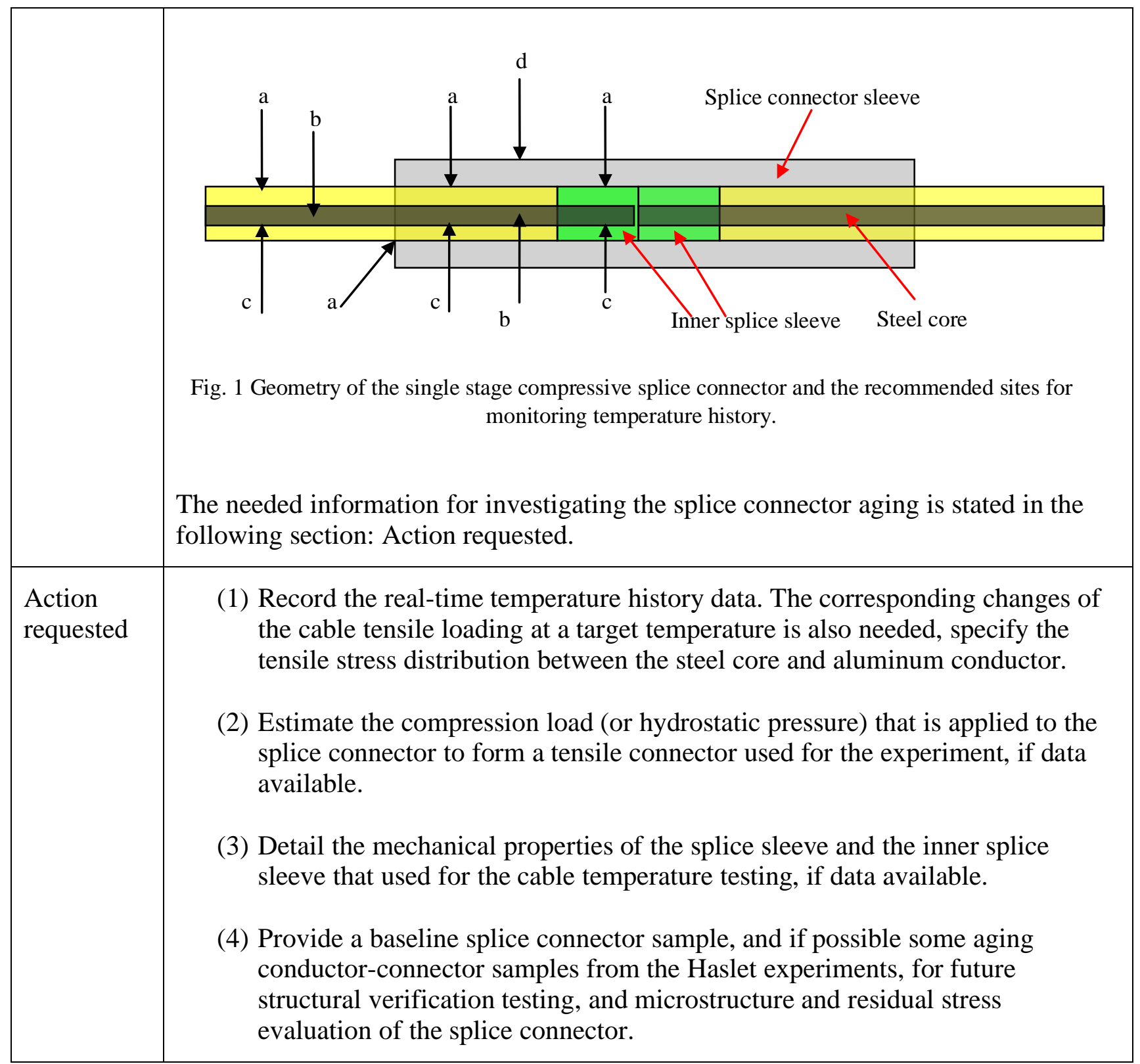

Table 2: Resistivity measurement

\begin{tabular}{|l|l|}
\hline Title & Issues related to Resistivity Measurement \\
\hline Description & $\begin{array}{l}\text { The resistivity changes of a conductor-connector region can provide a first order (or } \\
\text { rough) index or a qualitative benchmark in regard to connector aging. The loss of } \\
\text { compressive residual stress within the connector and the microstructural evolution of } \\
\text { the conductor material-due to thermal mechanical fatigue and the creep effect, will } \\
\text { inevitably cause an increase of the resistivity in the conductor-connector system. } \\
\text { Thus, changes of resistivity can also provide an indirect calibration (index) for the } \\
\text { degradation of compressive stress within the connector during service. Therefore, I }\end{array}$ \\
\hline
\end{tabular}




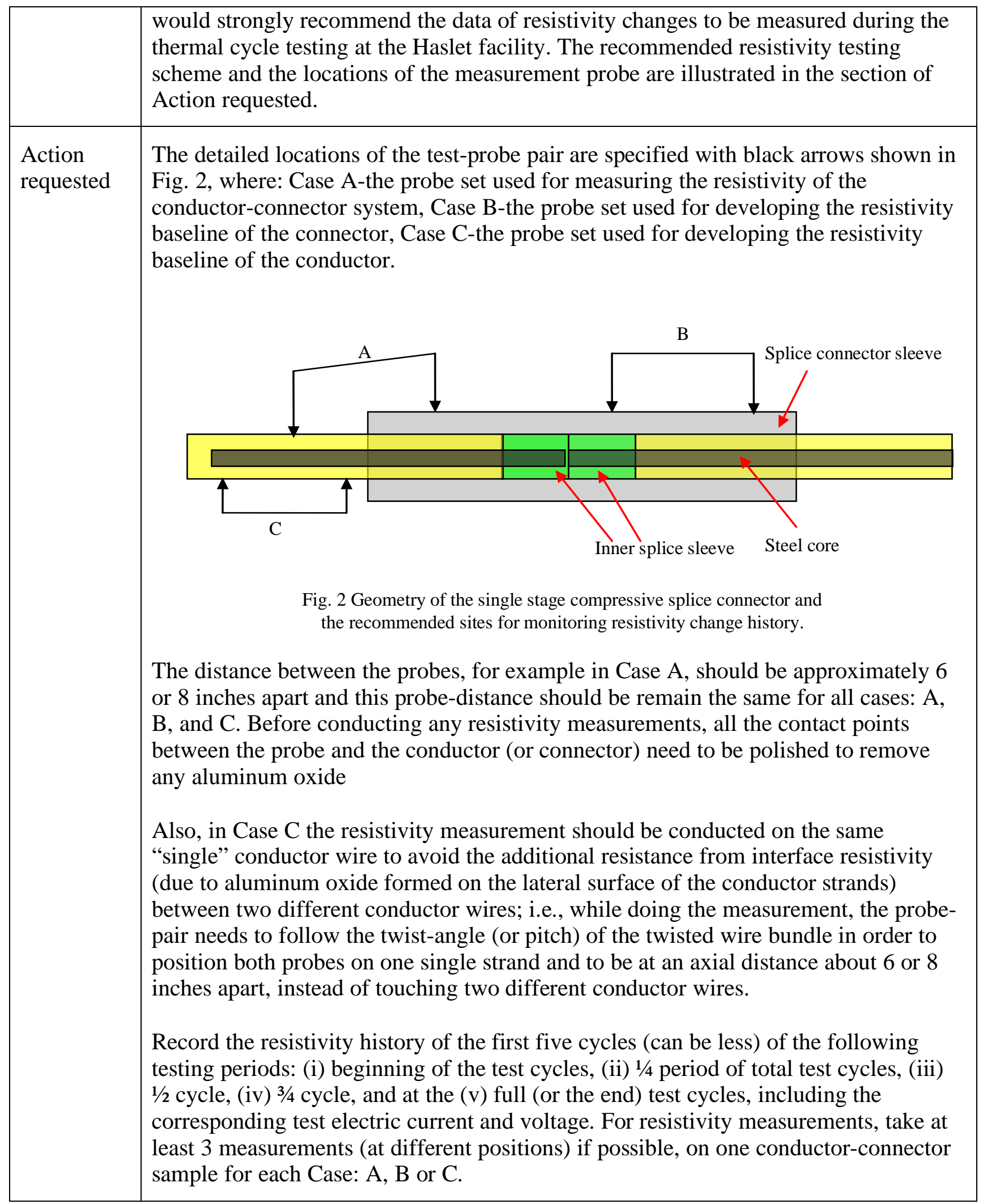

Table 3: Bending moment associated aging at suspension ends 


\begin{tabular}{|c|c|}
\hline Title & Bending Moment Issue \\
\hline Description & $\begin{array}{l}\text { It is more likely that the bending moment cycle fatigue will not be a concern for } \\
\text { an ACSR conductor-connector system operated at normal temperatures. } \\
\text { However, at suspension-ends, detailed scrutiny may need to be carried out to } \\
\text { examine the thermal cycle fatigue associated with alternation of bending stress } \\
\text { of an ACSR conductor operated at a higher temperature domain. }\end{array}$ \\
\hline \multirow[t]{3}{*}{$\begin{array}{l}\text { Action } \\
\text { requested }\end{array}$} & $\begin{array}{l}\text { (1) Detailed measurements of the initial cable length, sag and the associated } \\
\text { tension loading should be registered at the beginning of the test (at } \\
\text { ambient temperature), and monitored periodically during the test cycles } \\
\text { with different targeted temperatures. These data can provide benchmarks } \\
\text { for the conventional approach or force equilibrium approach used in } \\
\text { bending moment evaluation. } \\
\text { (2) For a continuing cable span structure, the maximum bending moment } \\
\text { occurs at the cable suspension ends. Thus, it warrants the investigation on } \\
\text { the bending at the suspension ends. The test configuration for such study } \\
\text { is recommended below. Use two suspension clamps anchored to the } \\
\text { conductor- between the two dead end splice connectors where the tension } \\
\text { loading will be applied. If this can be done, please also mount a pair of } \\
\text { Rosette strain gages on the top and bottom of the conductor near the } \\
\text { clamped end. Using a Rosette strain gage will provide further } \\
\text { information regarding the torsional shear deformation of the aluminum } \\
\text { conductor during heating. }\end{array}$ \\
\hline & $\begin{array}{c}\text { Supporting } \\
\text { structure }\end{array}$ \\
\hline & $\begin{array}{l}\text { (3) From the earlier study, it shows that relatively large bending moment can } \\
\text { be observed from a longer cable span structure at suspension ends, and } \\
\text { lesser bending moment for a higher tension loading. With the planned } \\
130 \mathrm{ft} \text { span used for the bending moment evaluation testing, the low per- } \\
\text { tension loading is more desirable, compared to using high tension loading } \\
\text { for investigating the bending effect. }\end{array}$ \\
\hline
\end{tabular}

